\title{
Analytical Methods to Investigate Occupational Exposure to Toxic Elements and their Species
}

\author{
Elizabeth Joanne Leese
}

A thesis submitted in part fulfilment of the requirements of Sheffield Hallam University for the degree of Doctor of Philosophy

In collaboration with the Health \& Safety Executive and the Health \& Safety Laboratory

June 2017 


\section{Acknowledgements}

I would firstly like to give my thanks and gratitude to my Director of Studies, Dr Vikki Carolan for her continued support, guidance and input throughout the past 7 years of this project. I would also like to extend my thanks to my other supervisors Dr Philip Gardiner but especially Dr Jackie Morton. Jackie, you have continued to motivate, support and guide me, over not only the past 7 years but the last 12 years whilst working at HSL. Your continued belief in my abilities and the opportunities you have given me, have made me the person and the scientist I am today and I will be eternally grateful. You continue to be my mentor and a good friend.

This PhD was funded by the HSE and HSL, so I would like to thank them and Prof Andrew Curran for giving me this opportunity.

I would also like to thank all the people who took part in this project, the staff at HSL and everyone at Sheffield Hallam University within the BMRC who provided either an EBC or urine sample (sometimes both) to form my unexposed control groups. I would especially like to thank the companies and workers who also volunteered to take part in each project. They very generously donated their time and welcomed me onto site to collect samples.

I must also thank Prof Malcolm Clench and Dr Craig Sams for all their help with the ESI and LC-MS work. In addition, another special thank you goes to Kate Jones for your continued help and support. You have listened to more than your fair share of either my excitable, forlorn or irate ramblings at the end of a long day.

I would finally like to thank my parents and family for your encouragement and continued belief in me. Most of all, I want to thank Vance for your support and confidence, you never doubted me for a second from the start of this journey that I could not do it. For looking after me and bringing me little treats and pick-me ups during the darkest days, but most of all for just being there. 


\begin{abstract}
$\underline{\text { Abstract }}$
With many occupations involving the use of chemicals considered hazardous to health, the use of biological monitoring to monitor and control exposure to chemicals may help to reduce the risk of ill health. However, this is not always possible for all elemental workplace exposures.

The work in this PhD thesis outlines the improvement of existing methodologies and, the development of novel speciation methodologies, including single particle analysis. It furthers biological monitoring research of elements that are of a carcinogenic occupational concern, i.e. inorganic arsenic, hexavalent chromium and respirable crystalline silica (RCS). Current biological monitoring methods are either no longer deemed fit for purpose (arsenic), not ideal (chromium) or are not available (RCS). The limitations of occupational exposure assessment of hexavalent chromium and RCS are linked to the unsuitability of biological matrices for biological monitoring purposes. Therefore, the work in this thesis also evaluates exhaled breath condensate (EBC) for its potential as a new biological matrix for biological monitoring of chromium and RCS.

The analytical technique used for arsenic speciation, chromium speciation and single particle analysis of RCS was inductively couple plasma - mass spectrometry (ICP-MS). Separation was achieved for the chromium and arsenic methodologies by hyphenating the ICP-MS with a micro liquid chromatography $(\mu \mathrm{LC})$ system coupled to an anion exchange column.
\end{abstract}

For arsenic speciation, a novel and robust 6-minute method was established. Additionally, a background levels study was conducted to establish an unexposed background reference range for five species of arsenic, to improve interpretation of biological monitoring results. Unexpected arsenic peaks in routine biological monitoring samples were also investigated, with one of which being determined as thio-DMA.

For chromium speciation, a novel method to simultaneously separate and detect both hexavalent and trivalent species in EBC was developed, incorporating a storage stability study of both species of chromium in EBC. This was followed by a feasibility study, which determined that both species of chromium can be detected and measured in 'real' EBC samples and that occupationally exposed workers showed significantly higher levels of both chromium species in EBC samples compared to an unexposed control group.

For RCS, the potential of utilising EBC to both detect and measure occupational exposure to RCS, employing the new and challenging analytical technique of single particle ICP-MS, was studied. The work demonstrated that individual particles of silica can be detected and counted in an EBC sample, with occupationally exposed workers showing significantly more RCS particles than an unexposed control group. 


\section{Contents}

Analytical Methods to Investigate Occupational Exposure to Toxic Elements and their Species.

Acknowledgements .ii

Abstract ... iii

Contents. iv

List of Tables viii

List of Figures ... ix

Abbreviations xi

Chapter 1. Introduction .1

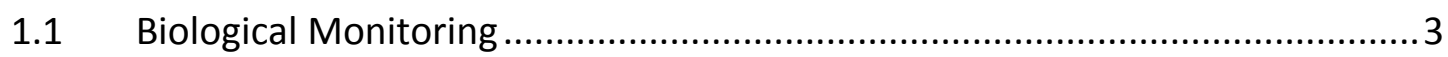

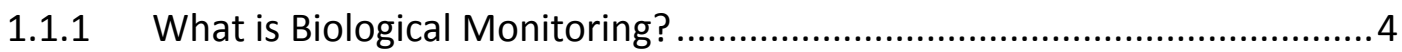

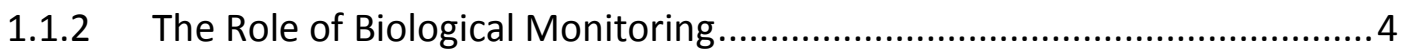

1.1.3 The Difference between Biological Monitoring, Biological Effect

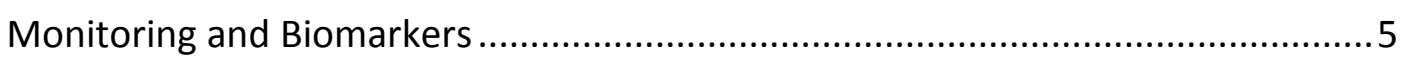

1.1.4 Biological Mechanism and Factors Affecting Exposure Assessment .........6

1.1.4.1 Kinetics - Dose, Half-Life, Distribution and Elimination ...................... 7

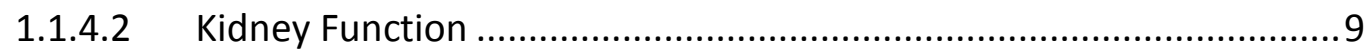

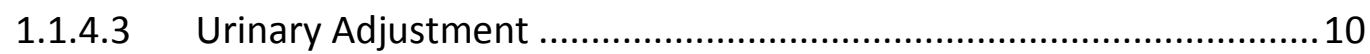

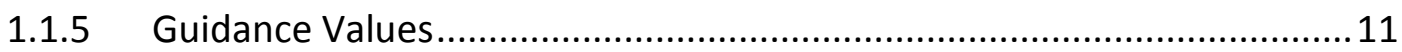

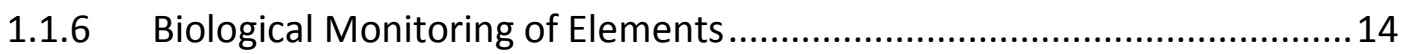

1.2 Exhaled Breath Condensate (EBC) ...................................................... 16

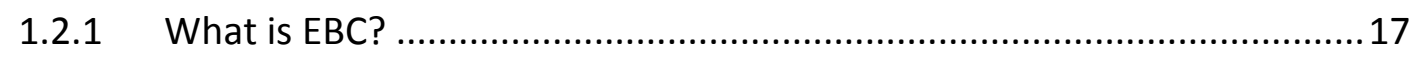

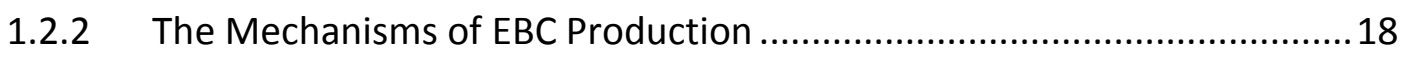

1.2.3 EBC Devices \& Collection Technique................................................. 19 
1.2.3.1 Devices......

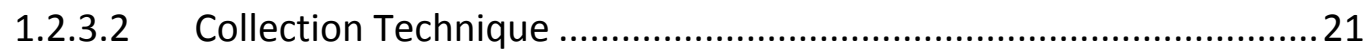

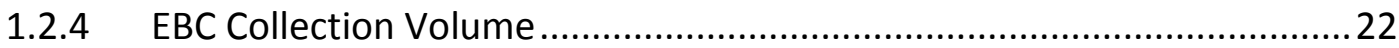

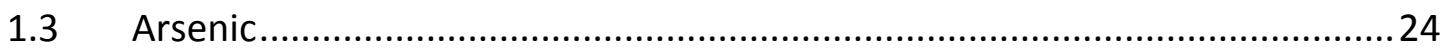

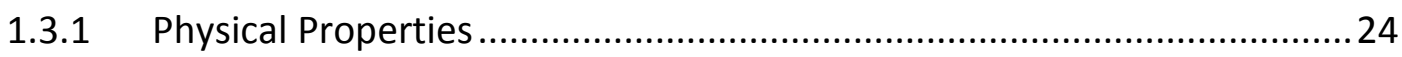

1.3.2 Arsenic Toxicity and Effects on Human Health ....................................24

1.3.3 Arsenic Compounds - Inorganic Arsenic..........................................28

1.3.4 Arsenic Compounds - Organic Arsenic ........................................... 28

1.3.4.1 Human Methylated Metabolites .....................................................28

1.3.4.2 Non-Human Occurring Metabolites .............................................30

1.3.4.3 New Generation of Oxo- and Thioarsenicals ................................ 31

1.3.5 Toxicity of Arsenosugars, Arsenolipids and their Respective Human

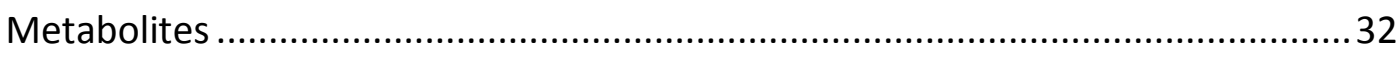

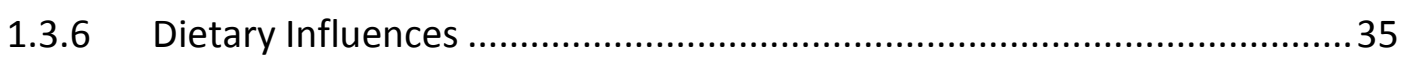

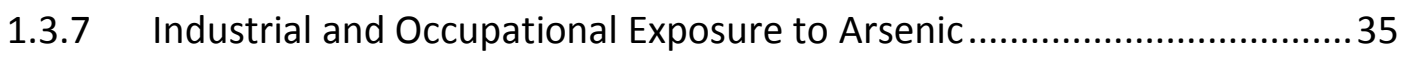

1.3.8 Biological Monitoring of Arsenic Exposure ...............................................

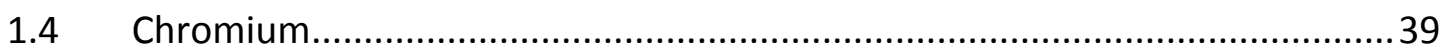

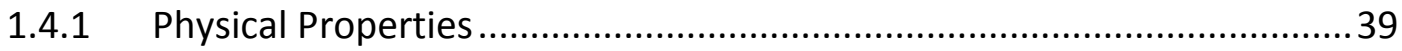

1.4.2 Chromium Toxicity and Effects on Human Health .................................39

1.4.3 Industrial and Occupational Exposure to Chromium .............................42

1.4.4 Biological Monitoring of Chromium Exposure .................................... 45

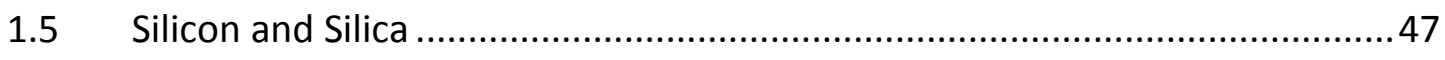

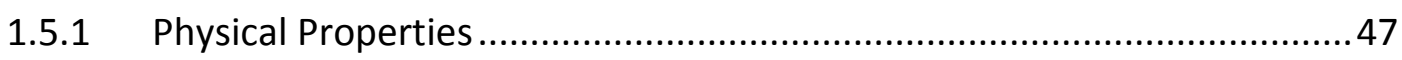

1.5.2 Silica Toxicity and Effects of Human Health....................................... 48

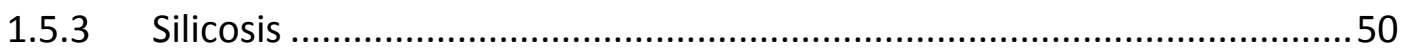

1.5.4 Industrial \& Occupational Exposure to Silica ...........................................51 


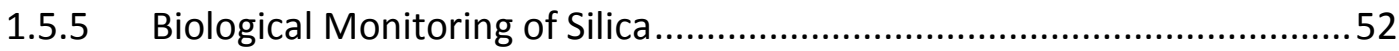

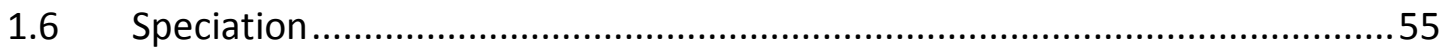

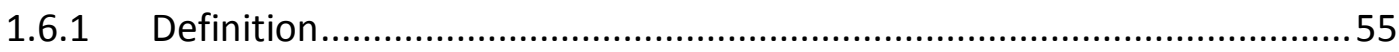

1.6.2 Speciation using Hyphenated Separation Techniques............................56

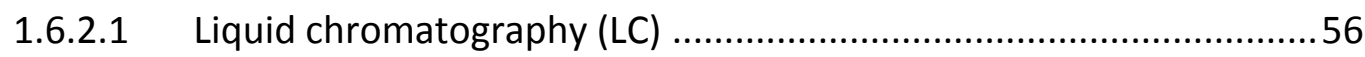

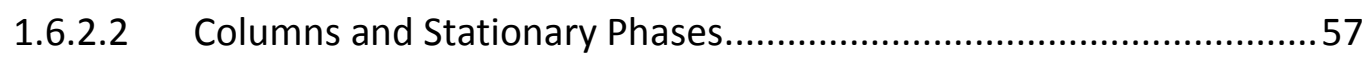

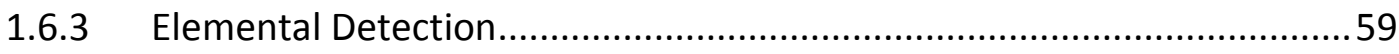

1.6.3.1 Inductively Coupled Plasma Mass Spectrometry (ICP-MS) ...............59

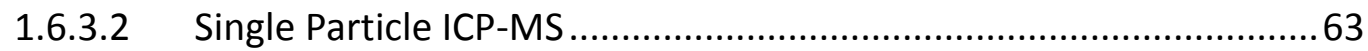

1.6.3.3 Tandem Mass Spectrometry - MS/MS ........................................66

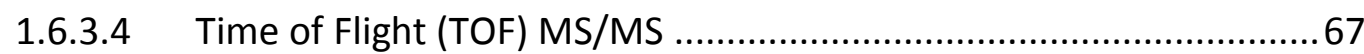

1.6.4 Chromatographic Column and LC System used in this Study..................68

1.6.4.1 Dionex ${ }^{T M}$ IonPac $^{T M}$ AG7 Anion Exchange Column ...........................68

1.6.4.2 Micro-flow Liquid Chromatography $(\mu \mathrm{LC})$ System ..........................68

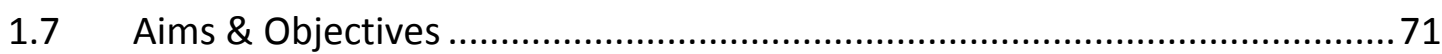

1.8 Publications Outlined in the Chapters of this Thesis ...................................72

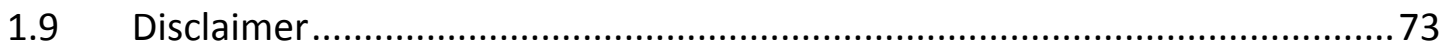

Chapter 2. $\quad \mu \mathrm{LC}-I C P-M S$ Determinations of Unexposed UK urinary Arsenic

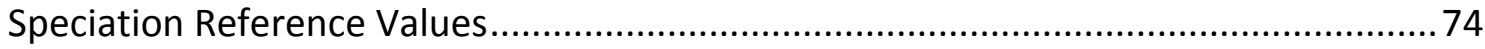

Chapter 3. The Investigation of Unexpected Arsenic Compounds Observed in Routine Biological Monitoring Urinary Speciation Analysis ...................................... 82

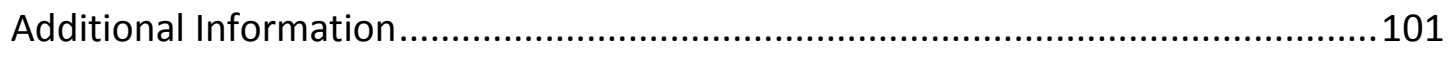

Chapter 4. Development of a Method for the Simultaneous Detection of $\mathrm{Cr}$ (III) and $\mathrm{Cr}(\mathrm{VI})$ in Exhaled Breath Condensate Samples using $\mu \mathrm{LC}-\mathrm{ICP}-\mathrm{MS}$. 103

Chapter 5. The Simultaneous Detection of Trivalent and Hexavalent Chromium in Exhaled Breath Condensate: A Feasibility Study Comparing Workers and Controls. ..114 
Chapter 6. Exhaled Breath Condensate: A Novel Matrix for Biological Monitoring to Assess Occupational Exposure to Respirable Crystalline Silica.....................................124

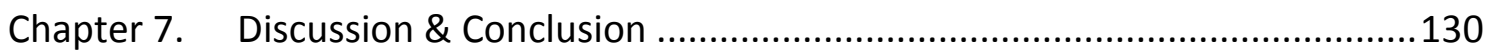

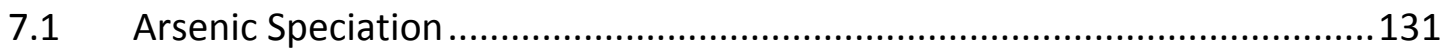

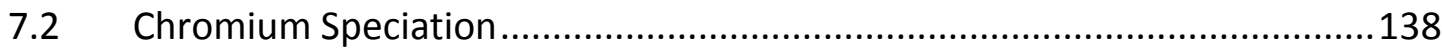

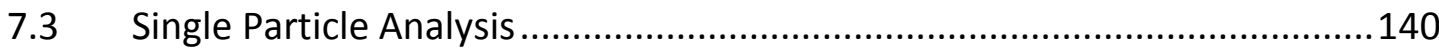

7.3.1 Advancements in Single Particle Analysis .........................................148

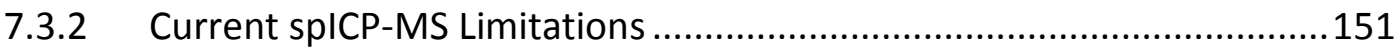

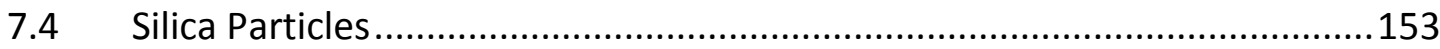

7.5 The Potential of other Elemental Single Particle Analysis ...........................157

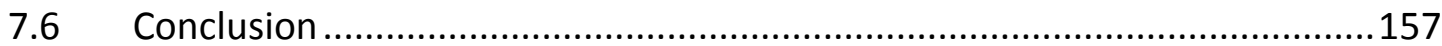

Chapter 8. Publications, Oral Presentations and Poster Presentations. ..................159

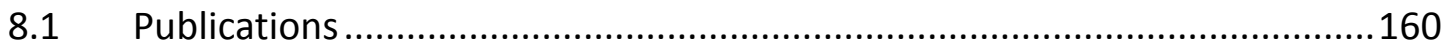

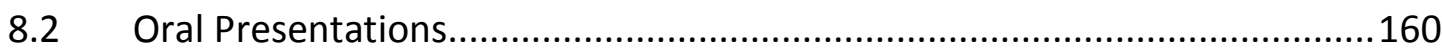

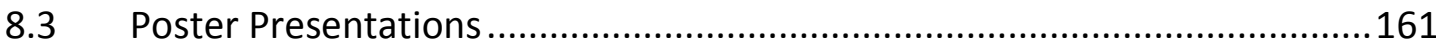

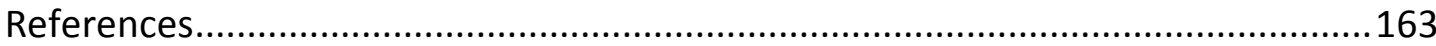

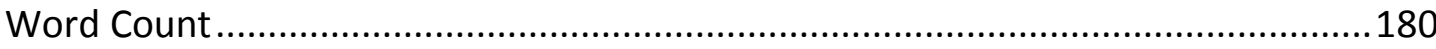

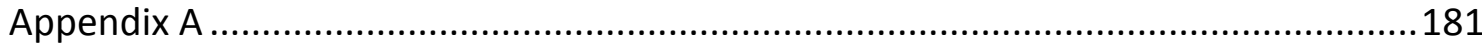




\section{List of Tables}

Table 1 The biological half-lifes of common occupational elements in urine

Table 2 Background reference ranges and BMGVs for a variety of elements considered carcinogens, mutagens and sensitisers in humans

Table $3 L_{50}$ values of arsenic compounds in laboratory experiments.

Table 4 Industries and occupations where exposure to inorganic arsenic occurs.

Table $5 \mathrm{LD}_{50}$ values of chromium compounds in laboratory experiments

Table 6 Industries and occupations where exposure to hexavalent chromium occurs. 42

Table 7 The purpose and use of specific compounds of hexavalent and trivalent chromium in different industries.

Table 8 The classification of silica forms 47

Table 9 Occupations and industries where exposure to RCS occurs

Table 10 Workplace activities that create RCS when working with crystalline silica containing rock, soil, sand, gravel, resources and products

Table 11 Examples of isobaric and polyatomic interferences of ions seen in ICP-MS applications.

Table 12 The $95^{\text {th }}$ and $75^{\text {th }}$ percentiles of different arsenic species reported in population studies around the World.

Table 13 A comparison of abilities and limitations of different analytical techniques to measure particles and nanoparticles 141 


\section{List of Figures}

Figure 1 Distribution and excretion pathways of an element in the human body after exposure by absorption, inhalation or ingestion. 9

Figure 2 The respiratory tract 18

Figure 3 The Turbo-DECCS exhaled breath condensate collection device. 21

Figure 4 Structures of arsenic compounds relevant to biological monitoring 27

Figure 5 The proposed classical pathway of inorganic arsenic biotransformation of oxidation methylation and reduction methylation steps in humans. 29

Figure 6 Arsenic compounds known as arsenosugars, arsenolipids and the metabolised dimethylated analogues after arsenolipid ingestion. 34

Figure 7 Block diagram showing components of a traditional LC system coupled to a detector. 57

Figure 8 A schematic diagram of a quadrupole ICP-MS with collision/reaction cell .....60

Figure 9 A schematic diagram of a plasma 61

Figure 10 Quadrupole rods within the mass analyser section of an ICP-MS 62

Figure 11 Time scan of a $1 \mu \mathrm{g} / \mathrm{L}$ ionic gold standard over a $60 \mathrm{~s}$ acquisition time, demonstrating the difference in the signal obtained by the detection of gold ions when the dwell time is $100 \mathrm{~ms}$ compared to $3 \mathrm{~ms}$ using spICP-MS. 65

Figure 12 Time scan of a $25 \mathrm{ng} / \mathrm{L} 30 \mathrm{~nm}$ gold particle certified reference material using spICP-MS. 65 
Figure 13 The oneFAST system comprising of a vacuum pump and switching valve unit (left). The oneFAST hyphenated to and, situated alongside the ICP-MS (right)

Figure 14 Switching valve positions of the oneFAST system, demonstrating uptake of mobile phase and sample

Figure 15 Comparative chromatograms showing the different retention times and ion transition ratios of a $0.1 \mathrm{mg} / \mathrm{L}$ standard of arsenocholine (top) and, a urine sample with unknown peak 1 (bottom). Ion transition ratios monitored were $\mathrm{m} / \mathrm{z}$ 165/122 and m/z $165 / 105$. 102

Figure 16 Sources of different categories of arsenic exposure and the resulting metabolites. Red compounds indicate the major metabolite from each source of exposure 136

Figure $17 \mathrm{~A}$ section of the RIKILT calculation tool spreadsheet showing the known criteria and result of the calculated nebulisation efficiency using a gold ionic calibration and $30 \mathrm{~nm}$ gold NIST CRM.

Figure 18 A section of the RIKILT calculation tool spreadsheet showing the known criteria and result from an RCS in-house (A9950) reference material approximately 50 $\mu \mathrm{g} / \mathrm{L}$

Figure 19 A QQQ ICP-MS/MS consisting of two mass filters and a collision/reaction cell situated in between, demonstrating its efficiency at removing nearly all polyatomic interferences, by applying $\mathrm{m} / \mathrm{z} 28$ to both mass filters. 149

Figure 20 Micrographs of two different respirable crystalline silica particles, demonstrating the irregularity in shape using a Tecnai G2 Spirit 120kV TEM with attached X-MAX energy dispersive spectroscopy system. 152 


\section{Abbreviations}

\begin{tabular}{|c|c|}
\hline AAS & Atomic Absorption Spectrometry \\
\hline$A B$ & Arsenobetaine \\
\hline$A C$ & Arsenocholine \\
\hline ACGIH & American Conference of Government Industrial Hygienists \\
\hline ALARP & As Low As Reasonably Practicable \\
\hline $\mathrm{As}^{3+}$ & Arsenite, Trivalent Arsenic \\
\hline $\mathrm{As}^{5+}$ & Arsenate, Pentavalent Arsenic \\
\hline ATSDR & Agency for Toxic Substances and Disease Registry \\
\hline \multirow[t]{2}{*}{ BAR } & Biologischer Arbeitsstoff-Referenzwert (German - Biological \\
\hline & Reference Value) \\
\hline \multirow[t]{2}{*}{ BAT } & Biologische Arbeitsstoff-Toleranzwerte (German - Biological \\
\hline & Tolerance Value) \\
\hline $\mathrm{BEI}$ & U.S. Biological Exposure Index \\
\hline BLW & Biologischer Leit-Wert (German - Biological Guide Values) \\
\hline BMGV & Biological Monitoring Guidance Value \\
\hline CE & Capillary Electrophoresis \\
\hline CLAW & Control of Lead at Work Act \\
\hline COPD & Chronic Obstructive Pulmonary Disease \\
\hline $\mathrm{COSHH}$ & Control of Substances Hazardous to Health \\
\hline CPS & Counts per Second \\
\hline $\mathrm{Cr}(\mathrm{III})$ & Trivalent Chromium \\
\hline $\mathrm{Cr}(\mathrm{VI})$ & Hexavalent Chromium \\
\hline DC & Direct Current \\
\hline DCS & Differential Centrifugal Sedimentation \\
\hline DFG & German Science Foundation \\
\hline DLS & Dynamic Light Scattering \\
\hline $\mathrm{DMA}^{3+}$ & Dimethylarsinite / Dimethylarsinous Acid \\
\hline $\mathrm{DMA}^{5+}$ & Dimethylarsinate / Dimethylarsinic Acid \\
\hline DMAA & Dimethylarsinoylacetate \\
\hline
\end{tabular}




\begin{tabular}{|c|c|}
\hline DMAE & Dimethylarsinoylethanol \\
\hline $\mathrm{EBC}$ & Exhaled Breath Condensate \\
\hline EDX & Energy Dispersive X-ray \\
\hline ESI & Electrospray Ionisation \\
\hline GC & Gas Chromatography \\
\hline GFAAS & Graphite Furnace Atomic Absorption Spectroscopy \\
\hline GFC & Glomerular Filtration Capacity \\
\hline GSH & Glutathione \\
\hline HSE & Health \& Safety Executive \\
\hline $\mathrm{HSL}$ & Health \& Safety Laboratory \\
\hline IARC & International Agency for Research on Cancer \\
\hline ICP-AES & Inductively Coupled Plasma Atomic Emission Spectrometry \\
\hline ICP-MS & Inductively Coupled Plasma Mass Spectrometry \\
\hline IUPAC & International Union of Pure and Applied Chemistry \\
\hline LC & Liquid Chromatography \\
\hline $\mathrm{LD}_{50}$ & $\begin{array}{l}\text { Lethal Dose - Lethal dose of a chemical to } 50 \% \text { of a given } \\
\text { population under defined set conditions }\end{array}$ \\
\hline $\mathrm{MMA}^{3+}$ & Monomethylarsonite / Monomethylarsonous Acid \\
\hline $\mathrm{MMA}^{5+}$ & Monomethylarsonate / Monomethylarsonic Acid \\
\hline MS & Mass Spectrometer/Spectrometry \\
\hline MS/MS & Tandem Mass Spectrometry \\
\hline$M / Z$ & Mass to Charge Ratio (amu) \\
\hline $\mathrm{N} / \mathrm{A}$ & Not Available \\
\hline NTA & Nanoparticle Tracking Analysis \\
\hline NTP & National Toxicology Program \\
\hline PIXE & Particle Induced X-ray Emission \\
\hline PPE & Personal Protective Equipment \\
\hline PTA & Particle Tracking Analysis \\
\hline RBC & Red Blood Cells \\
\hline RCS & Respirable Crystalline Silica \\
\hline $\mathrm{RF}$ & Radio Frequency \\
\hline
\end{tabular}




$\begin{array}{ll}\text { ROS } & \text { Reactive Oxygen Species } \\ \text { RPE } & \text { Respiratory Protective Equipment } \\ \text { RPS } & \text { Resistive Pulse Sensing } \\ \text { SAM } & \text { S-adenosylmethione } \\ \text { SEM } & \text { Scanning Electron Microscopy } \\ \text { SIOS } & \text { Scanning lon Occlusion Spectroscopy } \\ \text { spICP-MS } & \text { Single Particle Inductively Coupled Plasma Mass Spectrometry } \\ \text { TEM } & \text { Transmission Electron Microscopy } \\ \text { thio-DMA } & \text { Dimethylthioarsinate } \\ \text { thio-DMAA } & \text { thio-dimethylarsinoylacetate } \\ \text { thio-DMAE } & \text { thio-dimethylarsinoylethanol } \\ \text { TMAO } & \text { Trimethylarsine oxide } \\ \text { TOF } & \text { Time of Flight } \\ \text { TRA } & \text { Time Resolved Acquisition } \\ \mu \text { LC-ICP-MS } & \text { Micro Liquid Chromatography Inductively Coupled Plasma Mass } \\ \text { WEL } & \text { Spectrometry } \\ \text { WHO } & \text { Workplace Exposure Limit } \\ \text { ZPP } & \text { World Health Organisation } \\ & \text { Zinc Protoporphyrin }\end{array}$




\section{Chapter 1. Introduction}


This PhD thesis outlines the improvement of existing methodologies and the development of new speciation methodologies centred around the analytical technique, inductively coupled plasma mass spectrometry (ICP-MS), when investigating occupational exposure to toxic elements. The main elements and their species investigated are: arsenic, chromium and silicon. Each of these elements is challenging both analytically and from a biological monitoring approach because they exhibit different compounds and oxidation states of varying toxicity. In each method described the overall concept is for workplace exposure to be determined in a biological sample.

Arsenic speciation is the focus of Chapters 2 and 3. Chapter 2 summarises an optimised arsenic speciation method that is fast, reliable and robust for the analysis of routine biological samples using a novel $\mu \mathrm{LC}$ system coupled to an ICP-MS. Currently arsenic speciation results determined in a urine sample are difficult to interpret, as there are no guidance values for any of the individual species of arsenic. Neither are there defined levels of arsenic species in various foodstuffs nor how this affects arsenic species excreted in urine after ingestion. Chapter 2 focuses on the production of a background reference unexposed range for five species of arsenic in urine samples to aid interpretation of results from occupationally exposed individuals. The study also aims to provide a better understanding of dietary exposures and the types of food sources that may result in elevated urinary arsenic species. Arsenic speciation is an ever-evolving subject in terms of analytical and toxicological aspects. Therefore, Chapter 3 highlights the incidences of unexpected arsenic peaks in occupational routine urine samples and non-routine samples from hospital patients. The study outlines an investigation to identify these unexpected arsenic species and highlights the difficulties and limitations in this challenging area of arsenic speciation.

Chapters 4 and 5 focus on chromium speciation. Hexavalent chromium is of occupational concern because it is a carcinogen. Current biological monitoring of occupational exposure to hexavalent chromium is in the form of a urinary total chromium measurement, making it impossible to distinguish and quantify the essential trivalent form from the hazardous hexavalent species of chromium. Although 
occupational exposure results from three routes or pathways, inhalation is the primary route of exposure in many chromium industries and occupations. Exhaled breath condensate $(E B C)$ is a relatively under investigated biological matrix that represents occupational and environmental contaminants inhaled into the lungs, making it a potential biological matrix to assess occupational exposure. Chapter 4 demonstrates the first publication of the development of a method to simultaneously detect and measure both trivalent and hexavalent chromium in EBC samples using the a novel $\mu \mathrm{LC}$ system. In addition, a storage stability study of both species of chromium is presented. Chapter 5 is a feasibility study, incorporating this newly developed method, to determine if both species of chromium can be detected and measured in 'real' EBC samples from workers occupationally exposed to hexavalent chromium by inhalation and a control group.

Chapter 6 outlines the potential of utilising EBC to both detect and measure occupational exposure to respirable crystalline silica, employing the new and challenging analytical technique of single particle ICP-MS. Exposure to respirable crystalline silica is associated with lung diseases including emphysema, COPD, lung cancer and the terminal and irreversible condition known as silicosis. However, biological monitoring is currently unavailable for occupational assessment and so Chapter 6 highlights the potential of remedying this limitation.

\subsection{Biological Monitoring}

According to the Health and Safety at Work summary statistics 2016 provided by the Health \& Safety Executive (HSE) for Great Britain, during the 2015/2016 financial year 1.3 million workers were reported to be suffering from a work related illness. ${ }^{1}$ There are also an estimated 13000 annual deaths linked to past chemical or dust workplace exposure. It is believed that $36 \%$ of current annual deaths are related to asbestosrelated cancer, $33 \%$ are related to chronic obstructive pulmonary disease (COPD), $21 \%$ are related to other respiratory cancers and $10 \%$ to non-respiratory cancer. ${ }^{1}$ One objective of HSE is to reduce work-related death, injury and ill health. ${ }^{2}$ The Health \& Safety Laboratory (HSL) is HSE's laboratory and is one of the world's leading providers 
of health and safety solutions to industry, government and professional bodies, with the main focus of work on understanding and reducing health and safety risks. ${ }^{3}$

Potential hazards are present in all workplaces. One way HSL can help HSE to reduce work-related death, injury and ill health is by helping employers to protect employees by: monitoring and assessing exposure; testing and checking protective clothing and equipment; evaluating workplace equipment or personnel problems; and developing solutions to workplace problems. ${ }^{4}$ These deliverables are supported by an extensive scientific range of expertise. One area of workplace risk is occupational exposure to chemical substances and one practical approach to protecting employees and reducing workplace exposure is through biological monitoring.

\subsubsection{What is Biological Monitoring?}

Biological monitoring or biomonitoring can be defined as the assessment of systemic exposure to a chemical by the measurement of that chemical or its breakdown products (known as metabolites) in exposed individuals. ${ }^{5}$ There are three routes of exposure: inhalation; ingestion (usually by hand to mouth contamination); and dermal absorption through the skin. ${ }^{5}$ Biological monitoring measurements will reflect the total/combined uptake of a chemical by an individual through any of these exposure pathways or a combination of all three, from workplace, environmental and/or dietary exposures. ${ }^{5}$ Biological monitoring measurements are primarily undertaken in either a urine or whole blood sample, but can also be performed in other biological matrices such as breath, serum, plasma, hair, nail or other tissue samples. 5

\subsubsection{The Role of Biological Monitoring}

Biological monitoring is a measurement of exposure, not ill health. The use of biological monitoring in an occupational setting to monitor and control exposure to chemicals can subsequently help to reduce the risk of ill health. If long-term control was accomplished then it is feasible that a reduction in ill health would be achieved. 
Many occupations can involve the use of chemicals considered hazardous to health. The Control of Substances Hazardous to Health Regulations ${ }^{7}$ (COSHH) state that it is important that exposure to chemicals classified as carcinogens, mutagens and sensitisers be reduced to as low as is reasonably practicable (ALARP). In order to demonstrate that ALARP is in place, the workplace must be able to show evidence of their efforts to implement this viewpoint. Whilst this is a recommendation and biological monitoring is not compulsory (with the exception of lead exposure), ${ }^{8}$ it is one approach through measurement that can be used for assessing, reducing and controlling workplace exposure.

Biological monitoring offers an actual personal exposure measurement, differing from environmental monitoring, which measure an individual's potential exposure, for example by measuring a chemical in workplace air. The benefits of biological monitoring mean it can assess overall exposures (from three different routes) and provide an insight into the efficacy of personal protective equipment (PPE) or respiratory protective equipment (RPE), engineering controls and human factors.

Badham and Taylor first mentioned a form of biological monitoring in 1927 for lead in urine of exposed workers to help diagnose lead induced industrial disease. ${ }^{6}$ Since then, biological monitoring has been extensively used in numerous types of industries to help monitor workplace exposure to a wide range of chemical hazards, for example pesticides, solvents, isocyanates, metals and metalloids.

\subsubsection{The Difference between Biological Monitoring, Biological Effect Monitoring and Biomarkers}

Over recent decades, terms such as 'biomarkers of exposure' and 'biological effect monitoring' have been introduced to the scope of biological monitoring. Whilst biological monitoring is the measurement of the uptake of a chemical, biological effect monitoring relates to the measurement and assessment of biological effects as a result of the uptake. This usually involves the actual measurement of the biochemical effect. A biomarker of exposure is an indicator signalling a measure of exposure and a 
biomarker of effect is an indicator signalling a change to a biological function or a recognised possible health impairment or disease. For example, when monitoring exposure to inorganic lead (a chemical widely used by industries and occupations such as battery manufacture, lead paint production and use, foundries, roofing and glazing), the biological monitoring would be the quantitative determination of lead in a whole blood sample. The biological effect monitoring would be the measurement of zinc protoporphyrin (ZPP), because exposure to inorganic lead causes the inhibition of ferrocheletase, an enzyme that converts protoporphyrin into haem using the iron ion in red blood cells. ${ }^{9}$ This biochemical effect results in a zinc ion instead of a ferrous ion binding to protoporphyrin creating ZPP. This is a delayed action seen up to 2 months after the lead exposure. ${ }^{9}$ The measurement of ZPP in whole blood as a biomarker of effect can assist screening for lead exposure.

\subsubsection{Biological Mechanism and Factors Affecting Exposure Assessment}

As previously stated, any biological sample, fluid or tissue could be considered suitable for biological monitoring. However, blood and urine are the two biological samples most often used. ${ }^{6}$ This is especially true in Great Britain, as a non-invasive biological monitoring approach is favoured, ${ }^{10}$ meaning the majority of biological monitoring is based on urine samples. This approach also makes it easier for non-medically trained staff within workplaces to collect and send samples through the post. However, it is crucial to understand the pathways of uptake and excretion of a chemical, because sometimes a blood sample will be required when urine sampling is not considered appropriate. It is also important to understand dose, distribution, possible accumulation and metabolism of chemical substance after single or multiple exposures to fully evaluate and interpret biological monitoring results. In addition, individual behaviour and characteristics also have to be considered when evaluating results from different employees exposed to the same exposure. For example, the difference in speed and depth of breathing whilst performing workplace activities between individuals due to manual tasks or working in heat, the physiology and body mass of individuals in addition to age and gender will all influence uptake, metabolism and 
excretion. Furthermore, often being a smoker will increase the risk of hand to mouth contamination over non-smokers.

\subsubsection{Kinetics - Dose, Half-Life, Distribution and Elimination}

After exposure to a chemical, the amount that is taken in by inhalation, absorption or ingestion is called the dose, whilst the total concentration of that chemical found in the body, whether it be from the dose, accumulative doses or the background level concentration of a chemical found in a population, is termed the body burden. A biological half-life relates to the time required for the chemical substance in a biological system to reduce to half of its original value. ${ }^{11}$ The half-life can vary considerably depending on the chemical substance and also differs depending on the biological matrix, for example in blood, urine or organs. ${ }^{12}$ Compounds such as isocyanates can have a very short half-life of only 2 hours. ${ }^{13}$ As shown in Table 1 , elements tend not to have such short half-lives but can still vary greatly, for example cobalt has a half-life of 2-3 days ${ }^{9}$ whereas lead can be as long as 4 weeks. ${ }^{14}$ Biphasic or triphasic half-lives are known to exist, where an element has an initial rapid elimination followed by a second or third slower elimination.

\section{Table 1 The biological half-lifes of common occupational elements in urine(unless stated otherwise)}

\begin{tabular}{|c|c|c|}
\hline Element & Half Life & Ref \\
\hline Antimony & $\begin{array}{c}10-95 \text { hours } \\
20-40 \text { days }\end{array}$ & 9 \\
\hline $\begin{array}{c}\text { Arsenic } \\
\text { (Varied, depending on species) }\end{array}$ & $\begin{array}{c}20 \text { hours } \\
300 \text { hours }\end{array}$ & 15 \\
\hline Beryllium & Not fully understood & 9 \\
\hline $\begin{array}{c}\text { Cadmium in urine - } \\
\text { critical level from }\end{array}$ & Up to 30 years (liver \& kidney) & 14 \\
accumulation in liver \& kidney & & \\
\hline
\end{tabular}




\begin{tabular}{|c|c|c|}
\hline Element & Half Life & Ref \\
\hline Cadmium (blood) & 100 days & 14 \\
\hline Chromium & $\begin{array}{c}7 \text { hours } \\
15-30 \text { days } \\
3-5 \text { years }\end{array}$ & 16,17 \\
\hline Cobalt & $2-3$ days & 9 \\
\hline Lead (inorganic) & $3-4$ weeks (blood) & 14 \\
\hline Mercury & $1-3$ months & 18 \\
\hline $\begin{array}{c}\text { Mercury (blood) } \\
\text { Elemental \& inorganic } \\
\text { Methyl mercury }\end{array}$ & $\begin{array}{c}\text { 1-3 days } \\
1-3 \text { week } \\
50 \text { days }\end{array}$ & 18 \\
\hline Nickel & $17-53$ hours & 14 \\
\hline Platinum & $\begin{array}{l}50 \text { hours } \\
24 \text { days }\end{array}$ & 9 \\
\hline Selenium & $\begin{array}{c}1 \text { day } \\
8-20 \text { days } \\
65-116 \text { days }\end{array}$ & 9 \\
\hline Silicon (Silica) & $\begin{array}{l}\text { Ingested }-<24 \text { hours } \\
\text { Inhaled - unknown }\end{array}$ & 19 \\
\hline Thallium & $15-30$ days & 9 \\
\hline Titanium & Not fully understood & 20 \\
\hline Vanadium & $15-40$ hours & 9 \\
\hline
\end{tabular}

As shown in Figure 1 no matter the route of exposure, the blood is responsible for the circulation of the dose of the chemical substance around the body to the organs including the liver (the liver is the site of most elemental biotransformation, such as the methylation of arsenic) and ultimately excretion via the kidneys in urine. As the blood circulates the chemical around the body, the term critical organ relates to those 
organs where the first signs of an adverse effect can be detected. ${ }^{12}$ This adverse effect within the human body is also known as the critical effect. ${ }^{12}$

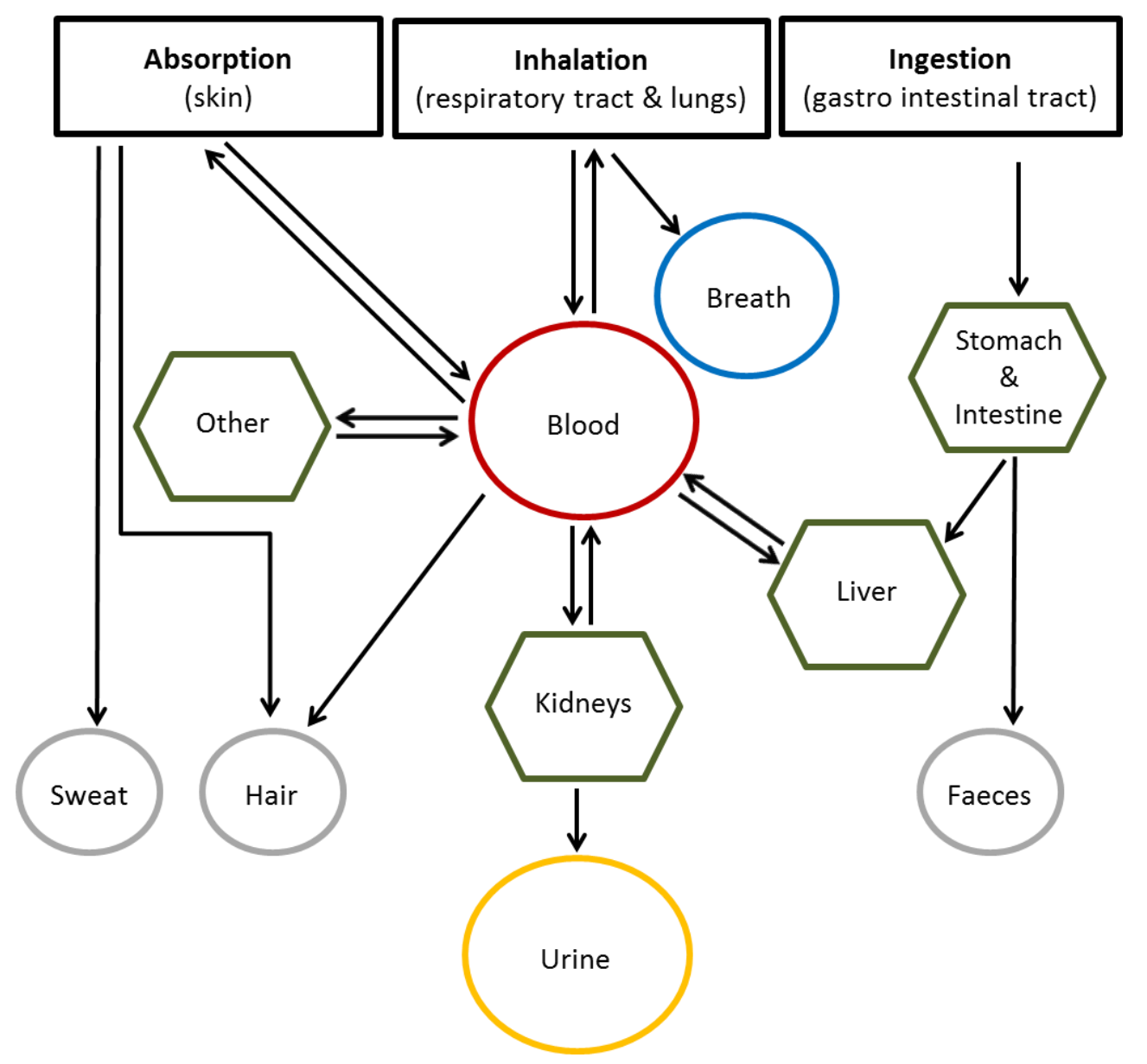

Figure 1 Distribution and excretion pathways of an element in the human body after exposure by absorption, inhalation or ingestion.

\subsubsection{Kidney Function}

The dose of a chemical substance eliminated in a urine sample is heavily influenced by kidney function and the degree of dilution. ${ }^{12}$ The kidneys are constantly producing urine by a complex system of filtration, reabsorption and elimination. From the blood flowing through the renal glomeruli, the glomeruli filter out water, salts, amino acids, sugars and small proteins into the renal tubules. ${ }^{6,12}$ Much of this filtrate known as primary urine is needed by the body, resulting in more than $99 \%$ being reabsorbed. ${ }^{12}$ The remaining water and other unwanted substances that have not been reabsorbed 
are eliminated from the body as urine. Some chemical substances such as cadmium and mercury are bound to small proteins causing them to be reabsorbed in the renal tubules instead of being eliminated in the urine. ${ }^{6}$ This reabsorption causes accumulation within the renal tubules. The elimination of cadmium in urine can be used as a biomarker of the critical organ. ${ }^{6}$ Renal dysfunction affecting glomerular filtration capacity (GFC) is a consequence of the critical concentration of cadmium, resulting in the excretion of cadmium in the urine. Furthermore, a biomarker of effect would be the measurement of retinol binding protein in a urine sample, ${ }^{9}$ with increased protein in the urine resulting in the kidney's inability to reabsorb it in the renal tubules being a suitable form of biological effect monitoring to assess cadmium exposure.

\subsubsection{Urinary Adjustment}

In a clinical setting 24 hour urine collections are normal practice. ${ }^{21}$ However, the only practical workplace approach to biological monitoring means spot urine collections either during or at the end of the working shift. This can result in a wide range of dilution from one spot urine collection to the next and amongst different individuals experiencing similar workplace chemical exposures. The analyte of interest measured in urine samples will be significantly affected by the urine concentration or dilution depending on the fluid balance of the individual. ${ }^{21}$ There are several approaches to this problem all involving a measurement of adjustment, however the two most common are creatinine correction and specific gravity. ${ }^{6}$ Specific gravity or relative density of urine corrects for difference in urine density i.e. changes in urine flow. ${ }^{6}$ Adjustment is calculated by specific gravity increment in relation to a reference density of either 1.018 or $1.024 .{ }^{6}$ The second and most common method of dilution adjustment, ${ }^{21}$ and the one favoured by HSL, is creatinine correction. Creatinine is related to muscle mass, as it is a metabolite of creatine (an important muscle energy store). ${ }^{21}$ The elimination of creatinine is entirely through glomerular filtration, with the production and excretion remaining relatively constant within an individual, increasing from childhood into adulthood, with a slow decline after the age of 65 years. ${ }^{21}$ Creatinine corrected 
results are expressed as moles of analyte per mole of creatinine or less often as grams of analyte per grams of creatinine. ${ }^{6}$ However, both methods have their limitations. When a urine sample is extremely dilute or concentrated, the correction is likely to not be as accurate as with a urine sample of equal fluid balance. At HSL, a urine sample with a creatinine concentration of less than $3 \mathrm{mmol} / \mathrm{L}$ or greater than $30 \mathrm{mmol} / \mathrm{L}$ is flagged as a possible unreliable result. Similar limitations can be used for specific gravity, when relative density of a urine sample falls outside the range of $1.010-$ 1.030. ${ }^{6}$ Because creatinine is dependent on muscle mass, women tend to have lower levels of creatinine than men, resulting in a different creatinine corrected estimate of dose in women than in men. ${ }^{6}$ However, from a biological monitoring approach most guidance values and reference ranges are expressed as either uncorrected concentrations or creatinine adjusted values. ${ }^{21}$ In addition, the biological monitoring of workplace exposure will eliminate the variation of creatinine production and the inaccuracies in creatinine adjustment observed in urine samples from children and those over 65 years of age.

\subsubsection{Guidance Values}

The correct interpretation of biological monitoring results is crucial in maintaining the effectiveness of its use in exposure assessment. The main method of interpreting results is to compare them to background reference ranges and guidance values. Different countries and organisations have different kinds of guidance values and the term 'biological monitoring guidance value' (BMGV) is an umbrella term to encompass them all. Workplace guidance values have been produced by the American Conference of Governmental Industrial Hygienists $(\mathrm{ACGIH}){ }^{22}$ the German Science Foundation $(D F G)^{23}$ and $\mathrm{HSE}^{24}$ in Great Britain. The different guidance values have different origins and can become confusing if not fully understood. Guidance values can be divided into health based and non-health based. Health based guidance values include the American produced Biological Exposure Indices (BEI), the German produced Biological Tolerance values (Biologische Arbeitsstoff-Toleranzwerte (BAT)) and several of HSE's BMGVs (for example, mercury). Health based guidance values are founded on 
biomarker levels not associated with ill health, but associated with airborne exposure. For example, both the BEI and BAT are based on an exposure-based value found after an 8-hour exposure at either an American or German airborne exposure limit. ${ }^{9,25}$ There is a selection of non-health based guidance values. The German Biologischer Leit-Wert (BLW) values are for substances that lack the evidence for a BAT value but where biological monitoring is considered useful and needed. In Great Britain the focus of HSE's non-health based BMGVs is a unique approach based on the idea of 'good occupational hygiene practice'. ${ }^{25}$ This has no link to health or airborne measurements, but is instead the $90^{\text {th }}$ percentile biological monitoring value taken from large-scale surveys of workplaces following good occupational practice to control exposure.

To determine if an individual is occupationally exposed it is important to ascertain the levels that chemical substances occur at in the non-occupationally exposed background population. HSL has established background unexposed reference ranges for many elements based on background level studies, ${ }^{26}$ in addition the German Biological Reference Value (Biologischer Arbeitsstoff-Referenzwert (BAR)) is based on the same premise. Unfortunately, many elements do not have established guidance values or unexposed reference ranges. Such elements include the individual species of arsenic, which is addressed in Chapter 2 of this thesis. The BMGVs and background reference ranges for some elements in addition to highlighting those that do not exist (listed as not available (N/A)) are shown in Table 2.

Table 2 Background reference ranges and BMGVs for a variety of elements considered carcinogens, mutagens and sensitisers in humans $\mathbf{s}^{9,22,23,26}$

\begin{tabular}{|l|c|c|}
\hline \multicolumn{1}{|c|}{ Element } & $\begin{array}{c}\text { Background Reference } \\
\text { Range }\end{array}$ & $\begin{array}{c}\text { Biological Monitoring Guidance } \\
\text { Values }\end{array}$ \\
\hline Antimony & $\mathrm{HSL}<0.4 \mu \mathrm{mol} / \mathrm{mol}$ creatinine & $\mathrm{N} / \mathrm{A}$ \\
\hline $\begin{array}{l}\text { Arsenic: } \\
- \text { Individual species } \\
-\Sigma \text { of inorganic \& } \\
\text { methylated species }\end{array}$ & $\begin{array}{c}\text { Established in Chapter } 2 \text { of this } \\
\text { thesis }\end{array}$ & $\mathrm{N} / \mathrm{A}$ \\
\hline
\end{tabular}




\begin{tabular}{|c|c|c|}
\hline Element & $\begin{array}{l}\text { Background Reference } \\
\text { Range }\end{array}$ & $\begin{array}{c}\text { Biological Monitoring Guidance } \\
\text { Values }\end{array}$ \\
\hline Beryllium & $\begin{array}{c}\text { BAR }<50 \mathrm{ng} / \mathrm{L} \\
\mathrm{HSL}<0.22 \mu \mathrm{mol} / \mathrm{mol} \text { creatinine }\end{array}$ & N/A \\
\hline $\begin{array}{l}\text { Cadmium } \\
\text { Critical Level }\end{array}$ & $\mathrm{HSL}<0.54 \mu \mathrm{mol} / \mathrm{mol}$ creatinine & $\begin{array}{c}\text { BEI } 4 \mu \mathrm{mol} / \mathrm{mol} \text { creatinine } \\
\text { BLW }<0.8 \mu \mathrm{g} / \mathrm{L}\end{array}$ \\
\hline Cadmium (Blood) & $\begin{array}{c}\text { HSL }<9 \mathrm{nmol} / \mathrm{L} \text { non-smoker } \\
\mathrm{HSL}<16 \mathrm{nmol} / \mathrm{L} \text { smoker } \\
\text { BAR } 1 \mu \mathrm{g} / \mathrm{L}\end{array}$ & BEI $5 \mu \mathrm{g} / \mathrm{L}$ or $45 \mathrm{nmol} / \mathrm{L}$ \\
\hline Chromium & $\begin{array}{c}\text { BAR }<0.6 \mu \mathrm{g} / \mathrm{L} \\
\mathrm{HSL}<2.9 \mu \mathrm{mol} / \mathrm{mol} \text { creatinine }\end{array}$ & $\begin{array}{l}\text { BEI } 25 \mu \mathrm{g} / \mathrm{L}(\sim 40 \mu \mathrm{mol} / \mathrm{mol} \text { creatinine }) \\
\text { HSE BMGV } 10 \mu \mathrm{mol} / \mathrm{mol} \text { creatinine }\end{array}$ \\
\hline Cobalt & $\mathrm{HSL}<2.5 \mu \mathrm{mol} / \mathrm{mol}$ creatinine & BEI $15 \mu \mathrm{g} / \mathrm{L}(\sim 20 \mu \mathrm{mol} / \mathrm{mol}$ creatinine $)$ \\
\hline $\begin{array}{l}\text { Lead inorganic } \\
\text { (Blood) }\end{array}$ & $<5 \mu \mathrm{g} / \mathrm{dL}$ & $\begin{array}{l}\text { CLAW suspension limits: } \\
60 \mu \mathrm{g} / \mathrm{dL} \\
30 \mu \mathrm{g} / \mathrm{dL}-\mathrm{WRA}^{*} \\
50 \mu \mathrm{g} / \mathrm{dL} \text { young people }\end{array}$ \\
\hline Lead organic & $\mathrm{HSL}<4.1 \mu \mathrm{mol} / \mathrm{mol}$ creatinine & $\begin{array}{c}\text { CLAW suspension limits } \\
60 \mu \mathrm{mol} / \mathrm{mol} \text { creatinine } \\
14 \mu \mathrm{mol} / \mathrm{mol} \text { creatinine - WRA* }\end{array}$ \\
\hline Manganese & $\mathrm{HSL}<1.45 \mu \mathrm{mol} / \mathrm{mol}$ creatinine & N/A \\
\hline Mercury & $\mathrm{HSL}<1.43 \mu \mathrm{mol} / \mathrm{mol}$ creatinine & $\begin{array}{c}\text { BAT } 14 \mu \mathrm{mol} / \mathrm{mol} \text { creatinine } \\
\text { BEI } 11 \mu \mathrm{mol} / \mathrm{mol} \text { creatinine } \\
\text { HSE BMGV } 20 \mu \mathrm{mol} / \mathrm{mol} \text { creatinine }\end{array}$ \\
\hline Mercury (Blood) & $\mathrm{HSL}<16 \mathrm{nmol} / \mathrm{L}$ & BLV $50 \mathrm{nmol} / \mathrm{L}$ \\
\hline Nickel & $\begin{array}{c}\text { BAR } 3 \mu \mathrm{g} / \mathrm{L} \\
\mathrm{HSL}<10.7 \mu \mathrm{mol} / \mathrm{mol} \text { creatinine }\end{array}$ & $\begin{array}{c}\text { BAT } 70 \mu \mathrm{g} / \mathrm{L}(\sim 100 \mu \mathrm{mol} / \mathrm{mol} \text { creatinine })- \\
\text { soluble nickel } \\
\text { BAT } 45 \mu \mathrm{g} / \mathrm{L}(\sim 60 \mu \mathrm{mol} / \mathrm{mol} \text { creatinine })- \\
\text { poorly soluble nickel }\end{array}$ \\
\hline Platinum & $\mathrm{HSL}<0.01 \mu \mathrm{mol} / \mathrm{mol}$ creatinine & $\mathrm{N} / \mathrm{A}$ \\
\hline Selenium & $\mathrm{HSL}<40 \mu \mathrm{mol} / \mathrm{mol}$ creatinine & $\begin{array}{c}\text { BAT }<150 \mu \mathrm{g} / \mathrm{L}(\sim 160 \mu \mathrm{mol} / \mathrm{mol} \\
\text { creatinine })\end{array}$ \\
\hline Silicon (Silica) & $\mathrm{N} / \mathrm{A}$ & N/A \\
\hline Thallium & $\mathrm{HSL}<0.26 \mu \mathrm{mol} / \mathrm{mol}$ creatinine & N/A \\
\hline Titanium & $\mathrm{HSL}<33.6 \mu \mathrm{mol} / \mathrm{mol}$ creatinine & N/A \\
\hline
\end{tabular}




\begin{tabular}{|l|c|c|}
\hline \multicolumn{1}{|c|}{ Element } & $\begin{array}{c}\text { Background Reference } \\
\text { Range }\end{array}$ & $\begin{array}{c}\text { Biological Monitoring Guidance } \\
\text { Values }\end{array}$ \\
\hline Vanadium & HSL $<10.7 \mu \mathrm{mol} / \mathrm{mol}$ creatinine & N/A \\
\hline
\end{tabular}

N/A - Not Available

*WRA - Women of reproductive capacity

\subsubsection{Biological Monitoring of Elements}

Metals and metalloids make up 100 of the 118 elements on the periodic table, ${ }^{27}$ of which 20 are known to be toxic. ${ }^{12}$ However, it is likely that other elements are also toxic but not enough scientific information/evidence has been gathered to categorise them as such. In contrast to this, many elements such as copper, iron, manganese, selenium and zinc are considered necessary for the everyday functioning of the human body. They have essential biological functions forming complexes with proteins and enzymes, for example iron, copper and zinc are associated with ferritin and copper with $\alpha$-amylase. ${ }^{28}$ The insufficient intake of an essential element will result in a deficiency however, toxic effects can occur if intake or exposure is high. Some elements have species of opposing potency such as chromium (trivalent chromium is an essential element whilst hexavalent chromium is toxic). After exposure and uptake of an element, it may transform into either a more toxic or less toxic species, but they do not break down in the human body, meaning they stay in the body until they are excreted. ${ }^{12,29}$ The vast majority of elements are completely eliminated in urine, hence being easily and accurately measured in a urine sample. However, elements such as mercury and cadmium, depending on the type of exposure are sometimes measured in a blood sample and workplace exposure to inorganic lead legally requires the measurement of a blood sample as per the Control of Lead at Work Act (CLAW). ${ }^{8}$ As previously stated in section 1.1.2, biological monitoring is one way that workplaces can demonstrate the exposure practice of ALARP to chemicals categorised as carcinogens, mutagens and sensitisers. Elements considered carcinogenic to humans ${ }^{30-32}$ are arsenic, beryllium, chromium, lead and nickel, whilst elements such as cobalt, iron, platinum, selenium, titanium and vanadium are classified as "reasonably anticipated to be $^{\prime 32}$ or "probably/possibly" ${ }^{31}$ carcinogenic to humans by the Agency for Toxic 
Substances and Disease Registry (ATSDR) or the International Agency for Research on Cancer (IARC) respectively. Mutagenic elements include cadmium, mercury and chromium, $^{33,34}$ whilst elements such as antimony, beryllium, chromium, cobalt, manganese, nickel, selenium and tin are considered dermal or respiratory sensitisers. ${ }^{32}$

As previously stated, blood and urine are the two biological samples most frequently used for occupational exposure assessment. However, in recent decades the interest in breath analysis in the form of exhaled breath condensate specifically within the field of airway and lung inflammation has expanded tremendously. ${ }^{35}$ As the primary route of occupational exposure for many industries is by inhalation, it is possible that exhaled breath condensate could be useful as a biological matrix for biological monitoring purposes, especially for elements where inhalation exposures are not fully understood and where biological monitoring is currently not possible. 


\subsection{Exhaled Breath Condensate (EBC)}

The potential for breath analysis as a diagnostic tool was identified as early as $\mathbf{1 7 8 4}$ when Antoine Lavoisier proved the consumption of oxygen and production of carbon dioxide during each exhalation of breath. Nearly 100 years later, observations were made by Anstie in 1874 of alcohol excretions in breath. ${ }^{36}$ The link between diabetes and acetone in exhaled breath was demonstrated in 1887 by Nebelthau. ${ }^{36}$ In the early 1900 s the development of alcohol breath tests was started, with the first patented device in $1931 .^{36}$

However, in modern day breath analysis there are two areas of breath research; those which concentrate on the gaseous phase of breath and volatile organic compounds, for example the electric nose and those which concentrate on the aqueous part of breath, known as exhaled breath condensate (EBC).

Inhalation is one of the primary routes of occupational exposure, with numerous inhalation exposures leading to inflammation and oxidative stress causing occupational asthma, various respiratory diseases and cancer. ${ }^{20}$ It is thought that EBC might be a useful biological monitoring matrix where either biological monitoring is currently not possible using traditional biological matrices such as urine or blood (for example respirable crystalline silica exposure) or where the interpretation of elemental species is difficult in a biological sample (for example hexavalent and trivalent chromium). It may also further the understanding of inhalation exposures and how elements behave and reside in lungs.

Scientists in the former Soviet Union were the first to report (in Russian) about EBC over 35 years ago. ${ }^{37}$ It took until 1992 for the first EBC paper to be published by Western Scientists. ${ }^{38}$ A publication by Dodig and Cepelak $^{39}$ in 2013 stated that between the period of 1980 - 2012 there were 869, 917 and 3628 published articles on EBC listed on databases PubMed, OVID and Scopus respectively. However, the vast majority of EBC publications have a strong emphasis on pulmonary disease diagnosis, with those who report a biomarker measurement focused on the study of specific 
respiratory diseases such as asthma, ${ }^{40-44}$ chronic obstructive pulmonary disease, ${ }^{40,44-46}$ silicosis, $^{47-50}$ asbestosis, ${ }^{47,50,51}$ obstructive sleep apnea, ${ }^{52}$ cystic fibrosis ${ }^{43,53,54}$ adult respiratory distress syndrome ${ }^{55}$ and lung cancer. ${ }^{56}$ Biomarkers of interest in these studies represent oxidative stress and inflammatory derived biomarkers, such as cytokines, $^{56,57}$ isoprostanes, ${ }^{48,49,58,59}$ leukotrienes, ${ }^{48,58,60} \mathrm{pH}$ changes, ${ }^{42,61}$ nitrogen reactive species such as nitrates/nitrites and nitrotyrosineoxides, ${ }^{42,43,46}$ hydrogen peroxide, ${ }^{41,42,62,63}$ and aldehydes. ${ }^{47,49,59,62,63}$ These biomarker studies all investigate potential applications in: the discrimination of different respiratory inflammatory types, early diagnosis of disease, detecting the severity of airway inflammation, the monitoring of treatment or disease control and the ability to predict disease exacerbation. ${ }^{64}$ Only a handful of publications have investigated EBC as a potential biological monitoring matrix to monitor occupational exposure to elements such as chromium, ${ }^{65-68}$ nickel and iron, ${ }^{68}$ chromium, nickel and aluminium, ${ }^{69}$ beryllium, ${ }^{70}$ lead, ${ }^{71}$ cobalt and tungsten, ${ }^{72,73}$ manganese $^{74}$ and potassium, cadmium, copper, antimony, zinc and strontium. ${ }^{75,76}$

\subsubsection{What is EBC?}

$E B C$ is a biological fluid. It consists mainly of water vapour but also small droplets of airway lining fluid from within the bronchial and alveoli regions of the lungs. However, it can also contain droplets from the entire respiratory tract (as shown in Figure 2) including the mouth and trachea. ${ }^{77}$

Within these droplets of airway lining fluid, is an unknown fraction of both volatile and non-volatile substances. Volatile substances are in the form of water-soluble compounds in the condensed water of the EBC sample such as ammonia, hydrogen peroxide and ethanol. ${ }^{78,79}$ Non-volatile substances can include cytokines, lipids and salts ${ }^{78}$ but also environmental and occupational contaminants. 


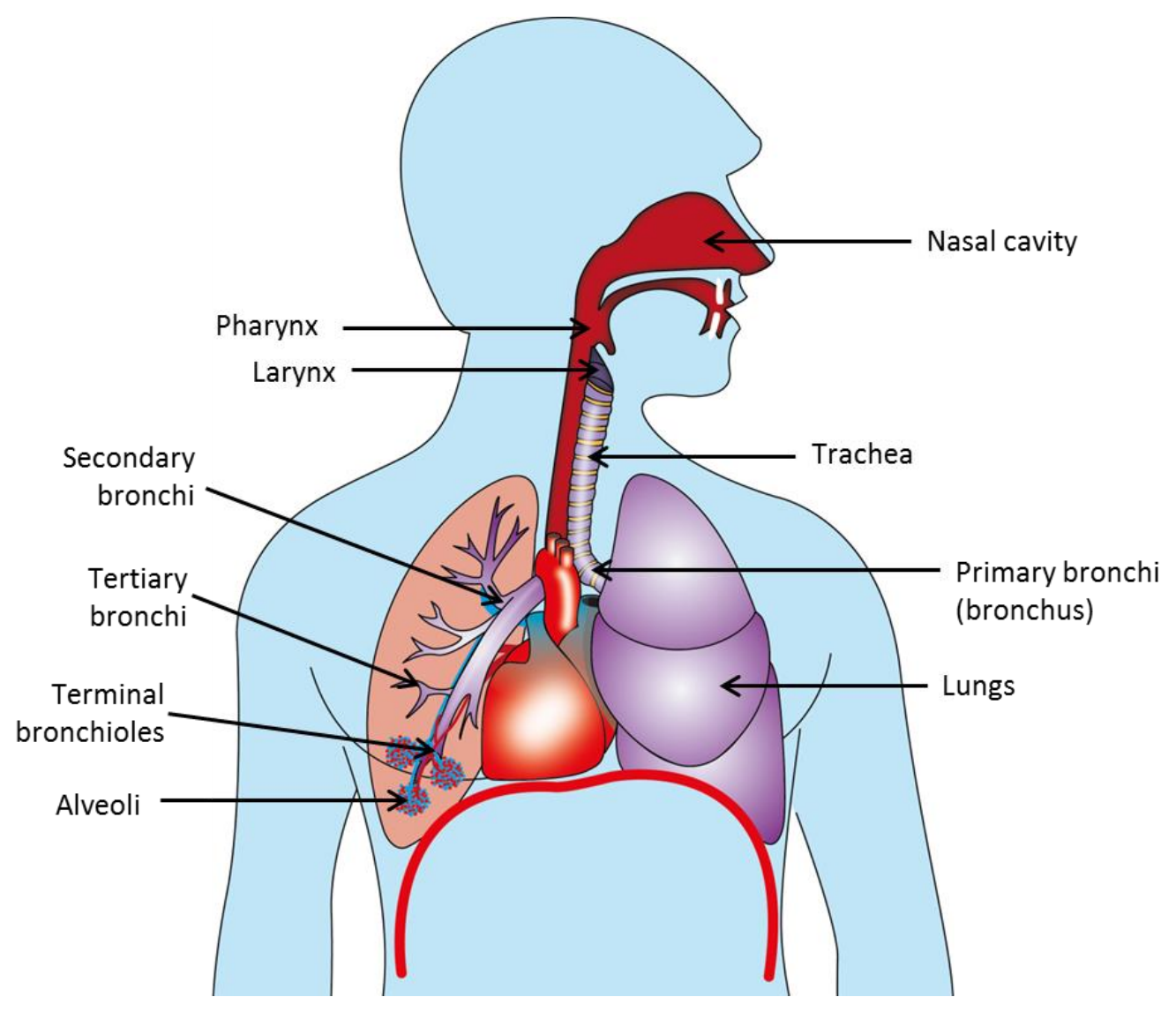

Figure 2 The respiratory tract created by HSE

\subsubsection{The Mechanisms of EBC Production}

The respiratory epithelium is lined with airway lining fluid. The exact mechanism of the aerosolisation of the airway lining fluid is not fully understood ${ }^{80}$ however, two possible models exist. The first model is termed 'turbulent airflow'. During regular tidal breathing, a turbulent airflow is created in the respiratory tract due to the opening of closed bronchioles and alveoli, causing droplets of the airway lining fluid containing non-volatile substances to become aerosolised. ${ }^{39,81}$ The second model is termed 'bronchiole fluid film burst' and was proposed in 2009 by Johnson \& Morawska. ${ }^{82}$ The elastic bronchioles have a surface consisting of longitudinal folds, which are lined with epithelial lining fluid. During exhalation, these folds disappear as the bronchiole contract and the fluid is expelled. This process continues until a flat, thin film of this fluid is formed over the surface blocking the expanding bronchiole, as the bronchioles open during inhalation a bubble is created which bursts in droplet aerosols that are 
held in the alveoli until exhalation. The bronchiole fluid film burst mechanism seems the likelier of the two concepts due to inaccuracies in the turbulent airflow model when respiratory conditions other than regular breathing are applied. For example, increased breathing or coughing, which should increase turbulent airflow, does not increase aerosol formation. ${ }^{82}$ In addition, the Johnson \& Morawska study determined that the aerosol formation increased only through increased inhalation rate and not during increased exhalation rate, proving that the aerosol formation is produced during the inhalation phase of breathing. ${ }^{82}$

The respiratory zone is a term used to describe the regions of the lungs which incorporate the respiratory bronchioles, alveolar ducts and alveoli. The airway lining fluid from the respiratory zone contains approximately $80 \%$ phospholipids, $10 \%$ cholesterol, $10 \%$ proteins and a very small amount of carbohydrate, which corresponds directly with the composition of EBC but in lower concentrations. ${ }^{39}$

\subsubsection{EBC Devices \& Collection Technique}

All EBC collection devices are based on a freezing cooling chamber, to cool and condense the exhaled breath, consisting of an inert material for the surface of the condensing cooling chamber such as glass, aluminium or Teflon as recommended by the American Thoracic Society/European Respiratory Society Task Force. ${ }^{81}$ The effectiveness of EBC collection depends on the volume of breath passing into the condenser and the temperature gradient of the exhaled air to the cooling chamber.

\subsubsection{Devices}

Prior to the availability of commercial EBC collection devices, research groups devised their own custom-built EBC collection equipment. The custom built devices have included: immersing a Teflon lined tube in an ice filled bucket to create a cooling chamber $^{61}$ and a double glass wall which icy water can circulate through as the EBC collected on the walls of the glass, subsequently dripping into a collection container below. ${ }^{83}$ These laboratory limitations resulted in the construction of in-house portable 
devices, some of which included breathing through a straw into a polypropylene tube cooled by ice ${ }^{84}$ or cooling gel. ${ }^{42}$ These in-house devices are termed 'open-end' meaning there is no recirculation, which results in a collection efficiency of only $50 \%{ }^{36}$ The commercially available EBC collection devices used in studies within the literature are described below.

The EcoScreen, (Erich Jaeger $\mathrm{GmbH}$, Hoechberg, Germany) (now discontinued) and the EcoScreen2 (FILT, Berlin, Germany). ${ }^{85}$ The EcoScreen was a device using a one-way tubing system, saliva trap and a reusable cooled PTFE coated aluminium double lumen lamellar system, with a disposable polypropylene collection cup. The cooling system was electrically cooled by a standard electrical connection that froze the EBC sample during collection. The EcoScreen2 now collects EBC in a disposable polyethylene bag. It also claims to have the ability of fractionated EBC collection in two separate chambers to distinguish between EBC from the mouth and upper respiratory tract and EBC from the lower respiratory tract. This device also has the added extra of a built in spirometer, enabling the measurement of exhaled volume. However, neither of the two EcoScreen devices is portable, being approximately the size of a desktop PC. This type of device is ideal for in-house laboratory research or hospital based research where volunteers are able to provide an EBC sample. However, they are not as suitable for biological monitoring EBC collection where sampling is usually completed postworking shift at the place of work.

RTube (Respiratory Research Inc., Charlottesville, VA, USA). ${ }^{86}$ The RTube is a portable device consisting of a disposable t-section containing a non-rebreathing valve and saliva separator. It also employs a syringe plunger to remove all EBC sample off the surface of the polypropylene collection tube. This device is considered completely portable, as it does not require a standard electrical connection to cool the condensing chamber. Cooling is achieved by the placement of an aluminium sleeve over the collection tube. However, this does mean that the sleeve will need to be chilled in a freezer or refrigerator prior to use and that numerous sleeves may be required for large or repeat collections, in addition to the temperature of the sleeve increasing over the duration of EBC collection. 
The TurboDECCS (Medivac SRL, Parma, Italy; previously by ItalChill). ${ }^{87}$ The TurboDECCS $^{-1}$ (Transportable Unit for the Research on Biomarkers Obtained from Disposable Exhaled Condensate Collections Systems) is the EBC collection device used to collect EBC samples in the work reported in chapter 4 and chapter 5 of this thesis. The TurboDECCS, as shown in Figure 3, is composed of two sections. The 'turbo' is the refrigeration device that incorporates a Peltier-cooling unit made of aluminium, which houses the EBC collection tube. The turbo requires a standard electrical connection to cool the condensing chamber however; the temperature is adjustable from $-10^{\circ} \mathrm{C}$ to room temperature. The 'DECCS' refers to the disposable polyethylene respiratory system comprising of a mouthpiece connected to a one-way aspiration valve with a saliva trap with an EBC collection tube inserted into the chilling unit as shown in Figure 3. Although the TurboDECCS is restricted to an electrical connection, unlike the EvoScreen, the unit is small and easily transported to different workplaces to collect samples.

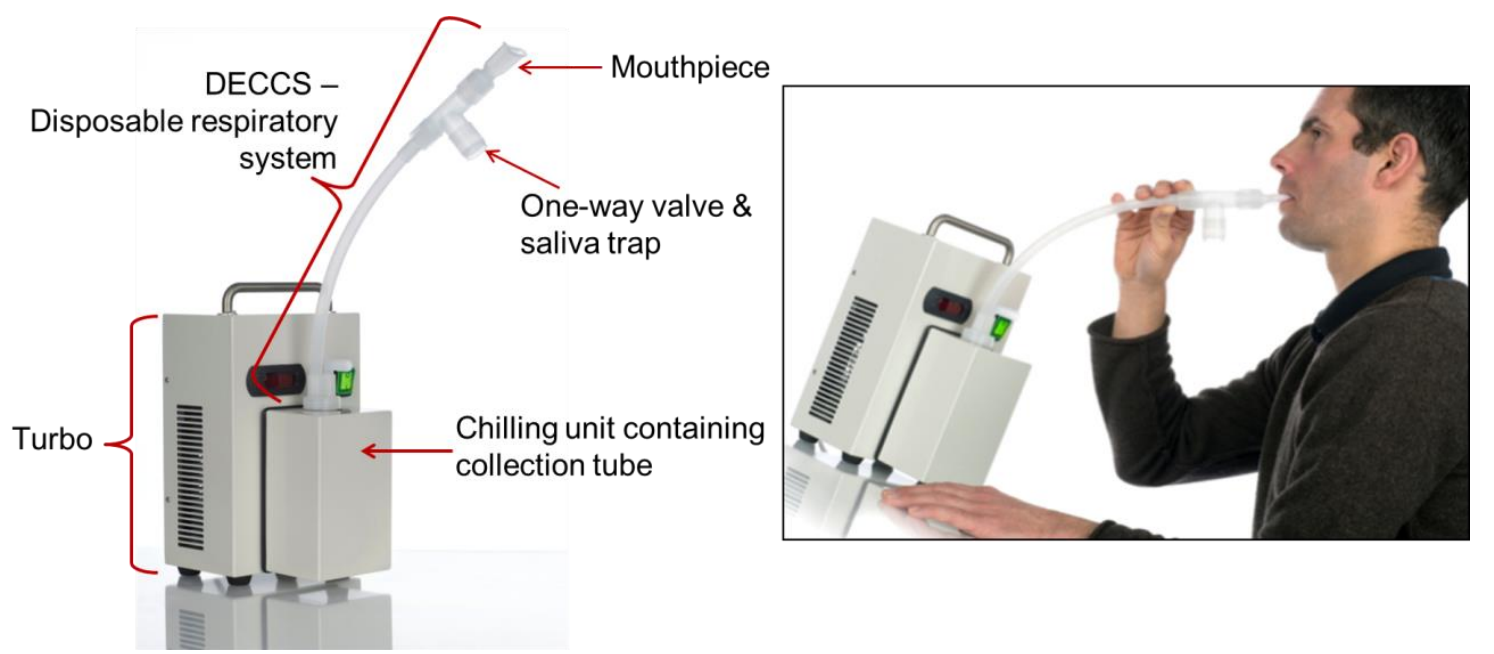

Figure 3 The Turbo-DECCS exhaled breath condensate collection device.

\subsubsection{Collection Technique}

The collection of EBC is non-invasive and the sampling of EBC does not cause an inflammatory response itself. ${ }^{88} \mathrm{EBC}$ is collected through regular, calm tidal breathing through the mouth estimated to be approximately 15-20 breaths per minute via a mouthpiece. ${ }^{39}$ The exhaled air produced during regular tidal breathing passes into the 
frozen cooling chamber (normally at temperatures between $-5^{\circ} \mathrm{C}$ to $-30^{\circ} \mathrm{C}$ depending on the manufacturer), where the EBC is cooled and collected as a condensate on the surface of the chamber or collection vessel.

Some methodologies suggest subjects wear a nose clip. A study by Hoffmeyer et $a l^{88}$ examining biomarkers of inflammatory response found no difference in $\mathrm{pH}$, nitrogen oxides and leukotriene B measurements for EBC samples collected from subjects with a nose clip than without, though when subjects wore a nose clip a slightly higher volume of EBC was collected. Wearing a nose clip will prevent subjects breathing through their nose and the mixing of nasal air with bronchial air. ${ }^{64}$ Nonetheless, the discomfort experienced by some individuals when wearing a nose clip has the potential to impede volunteer recruitment and increase volunteer dropout.

It is suggested that EBC samples must be immediately frozen at $-80^{\circ} \mathrm{C}$ until analysis, ${ }^{36,81}$ however this practice must be validated by a stability study of the biomarker or element of interest. Chapter 4 of this thesis highlights this issue, where the freezing of EBC samples containing chromium species affected the integrity of hexavalent chromium, causing it to deteriorate.

\subsubsection{EBC Collection Volume}

The volume of EBC volume collected can vary, with studies suggesting ten minutes of tidal breathing will collect an estimated $1-2 \mathrm{~mL} .{ }^{77}$ A number of suggestions has been made for the difference in EBC volume collected between individuals such as gender, age, fitness, smoking status and lung status. A study by Liu and Thomas ${ }^{44}$ investigated some of these suggestions by collecting EBC from healthy, asthmatic and chronic obstructive pulmonary disease individuals. They found EBC volume was not affected by age, gender, disease status or lung function of the individuals. The lack of correlation between $E B C$ volume and lung function ${ }^{55}$ is supported by numerous studies, as EBC volume is directly correlated to tidal volume and minute/ventilation volume. ${ }^{44,55,89}$ Tidal volume refers to the volume of air displaced in the lungs between normal inhalation and exhalation. In a young human adult this is thought to be approximately 
$500 \mathrm{~mL}$ per inspiration. Minute/ventilation volume refers to the amount of gas inhaled or exhaled from an individual's lungs in one minute. Individuals with higher minute/ventilation volume and/or higher tidal volume will produce more EBC. There is also a linear correlation between the volume of EBC produced and the length of collection time when breathing at a constant respiratory rate. ${ }^{81} \mathrm{~A}$ study by McCafferty et $a l^{89}$ reported reduced $\mathrm{EBC}$ volume when individuals were inhaling cooler and drier air.

The droplets of airway lining fluid can be considerably diluted by the condensed water vapour in EBC samples, which has been reported to only affect the measurement of non-volatile substances. ${ }^{64}$ This dilution will vary between individuals, as differences in dilution will depend on the size and number of droplets of lining fluid during regular tidal breathing. Unfortunately, there is no gold standard dilution marker for EBC. ${ }^{77}$ It has been suggested that a stable compound in blood which can diffuse through cell membranes in the lungs but is not produced itself in the alveoli or respiratory tract is a promising possibility to standardise EBC dilution. ${ }^{36}$ The biomarkers to correct for dilution which have been investigated include urea, $^{55,90}$ total protein content, ${ }^{55}$ ions/electrolytes (potassium, chloride, ammonia) 53,91,92 and the conductivity of Iyophilised samples. ${ }^{91}$ Until a suitable biomarker of dilution correction has been found the correlation between tidal volume and minute/ventilation volume with EBC volume suggests that $\mathrm{EBC}$ results should be reported per volume of $\mathrm{EBC}{ }^{55}$ 


\subsection{Arsenic}

\subsubsection{Physical Properties}

Arsenic is a metalloid residing directly below phosphorous in group 15 of the period table. Arsenic has both metallic and non-metallic properties and, in biological systems, arsenic can often substitute for phosphorous because of its close chemical characteristics. Arsenic has the atomic number 33 and a mass of 74.922 amu. It is mono-isotopic and is an ubiquitous element found in all rock, soil, water and air at trace levels. ${ }^{93}$ It is ranked as the $20^{\text {th }}$ most abundant element in the earth's crust, $14^{\text {th }}$ in seawater and $12^{\text {th }}$ in the human body. ${ }^{94}$ More than 200 identified minerals are found to contain arsenic such as realgar, orpiment and arsenolite with the most abundant arsenic containing mineral being arsenopyrite. ${ }^{93,94}$

Arsenic displays several valence states: arsine (-3); elemental (0); arsenite (+3) and arsenate $(+5)$. There are over fifty identified arsenic compounds or species all of which display very different levels of toxicity. ${ }^{95}$

\subsubsection{Arsenic Toxicity and Effects on Human Health}

Arsenic toxicity is dependent on its species and how each species is metabolised in the human body. ${ }^{96}$ However, the toxicity of arsenic and its carcinogenicity is still not fully understood. The variety of arsenic species provide a range of toxicological behaviours and, as arsenic undergoes rapid metabolism in the human body after exposure, it is difficult to differentiate which effects come from which species. Arsenic species of varying toxicities are shown in Figure 4.

Arsenic compounds can be categorised by: inorganic and organic compounds, with organic compounds composed of species that can be metabolised by humans and those that cannot. Inorganic arsenic compounds are considered to be more toxic than organic compounds. ${ }^{94}$ The US National Toxicology Program (NTP) has classified inorganic arsenic as a "human carcinogen"97 and IARC has classified inorganic arsenic 
as a Group 1 agent, defined as "carcinogenic to humans". ${ }^{31}$ A common way of evaluating the toxicity of a substance is the $L_{50}$ value. The International Union of Pure and Applied Chemistry (IUPAC) definition of an $\mathrm{LD}_{50}$ is the statistically derived dose of a chemical expected to kill $50 \%$ of a given population under defined conditions. ${ }^{11}$ However, these values expressed as unit mass of the toxic substance per unit mass of the test subject are not/rarely for humans and are usually for an oral ingested dose. The estimated lethal dose for humans ingesting inorganic arsenic is $1-3 \mathrm{mg} / \mathrm{kg}$ per day, however inhalation or skin absorption exposures to inorganic arsenic are not associated with acute lethal doses, ${ }^{98}$ unless in the form of arsine $\left(\mathrm{AsH}_{3}\right)$. Arsine is a gas which is the most acutely toxic arsenic compound. ${ }^{28}$ Table 3 outlines the LD $_{50}$ values for various arsenic compounds.

The adverse health effects caused by exposure to inorganic arsenic (arsenite $\left(\mathrm{As}^{3+}\right)$ and arsenate $\left.\left(A s^{5+}\right)\right)$ are vast, with health effects differing greatly between acute poisoning, long-term exposure and the route of exposure. The initial health effects from oral exposures and early signs of poisonings are nausea, vomiting and diarrhoea, abdominal pain and dehydration, ${ }^{99,100}$ with acute doses and poisonings resulting in confusion, hallucinations and an impaired memory, ${ }^{101}$ which in the long term can result in neurological effects. The most characteristic health effect from long-term acute oral exposures (commonly seen in arsenic contaminated drinking water) are skin manifestations as a result of vascular disease, ${ }^{101}$ such as hyperkeratinisation (excessive development of thick horny keratin in the epidermis) on the palms and soles of feet, cyanosis and gangrene most commonly known as Blackfoot Disease. ${ }^{100,101}$ However, unlike acute poisoning, there is no evidence of neurological effects from long term exposure to lower environmental or occupational levels of arsenic. ${ }^{100}$ Arsenic in drinking water is also related to increased cancer risks of the skin, lungs, bladder and kidney. ${ }^{100}$ The inhalation of inorganic arsenic results primarily in respiratory irritation of the mucous membrane which can lead to laryngitis, bronchitis, rhinitis and tracheobronchitis with higher exposures resulting in nasal septum perforation. ${ }^{102}$ Other health effects associated with arsenic exposure through either ingestion or inhalation exposures are hypertension, hypotension, cardiovascular problems, cardiomyopathy and anaemia. ${ }^{102}$ As a result of repeated exposures, arsenic is known 
to accumulate in both the kidney and liver over repeated exposure, meaning kidney

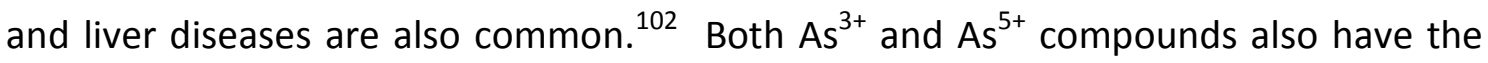
ability to be taken up by foetal blood and readily cross the placenta. ${ }^{93}$

Table 3 LD $_{50}$ values of arsenic compounds in laboratory experiments.

\begin{tabular}{|c|c|c|c|}
\hline Arsenic Compound & Test subject & LD50 (mg/kg) & Ref \\
\hline Arsine & Mice/Rat & $2-3$ & 103 \\
\hline \multirow[t]{2}{*}{ Arsenic trioxide } & Rat & 145 & 104 \\
\hline & Mice & 35 & 105 \\
\hline Calcium arsenite & Rats & 20 & 106 \\
\hline \multirow[t]{2}{*}{ Sodium arsenite } & Mice & 8 & 93 \\
\hline & Rat & 24 & 93 \\
\hline Potassium arsenite & Rat & 14 & 106 \\
\hline \multirow[t]{3}{*}{ Calcium arsenate } & Rat & 20 & 106 \\
\hline & Rat & 53 & 107 \\
\hline & Rat & $>400$ (dermal) & 107 \\
\hline \multirow[t]{2}{*}{ Lead arsenate } & Rat & 231 & 107 \\
\hline & Rat & >500 (dermal) & 107 \\
\hline Sodium arsenate & mouse & 21 & 93 \\
\hline DMA & Rat & $700-2600$ & 106 \\
\hline MMA & Rat & $700-1800$ & 106 \\
\hline Arsenobetaine & Rat & $>10000$ & 105 \\
\hline Arsenocholine & Rat & 6500 & 106 \\
\hline Trimethylarsine oxide & Rat & 10600 & 108 \\
\hline
\end{tabular}

Organic arsenic compounds occur when arsenic bonds with carbon. Compounds of organic arsenic vary in toxicity and this is primarily due to whether they are human metabolites of arsenic such as dimethylarsinate (DMA) and monomethylarsonate (MMA) or organic arsenic compounds that are not human metabolites such as arsenobetaine $(A B)$ and arsenocholine $(A C)$. 


\begin{tabular}{|c|c|}
\hline $\begin{array}{l}\text { Inorganic } \\
\text { Arsenic }\end{array}$ & $\begin{array}{l}\|_{\text {OH }}^{\mathrm{O}} \\
\mathrm{As}^{5+} \\
\text { Arsenate }\end{array}$ \\
\hline $\begin{array}{l}\text { Methylated } \\
\text { Metabolites }\end{array}$ & $\begin{array}{l}\mathrm{MMA}^{5+} \\
\text { Methylarsonate } \\
\text { Dimethylarsonate } \\
\text { DMA }\end{array}$ \\
\hline $\begin{array}{c}\text { Oxo- } \\
\text { dimethylated } \\
\text { Analogues }\end{array}$ & $\begin{array}{c}\text { DMAA } \\
\text { Dimethylarsinoylacetate }\end{array}$ \\
\hline $\begin{array}{c}\text { Thio- } \\
\text { dimethylated } \\
\text { analogues }\end{array}$ & 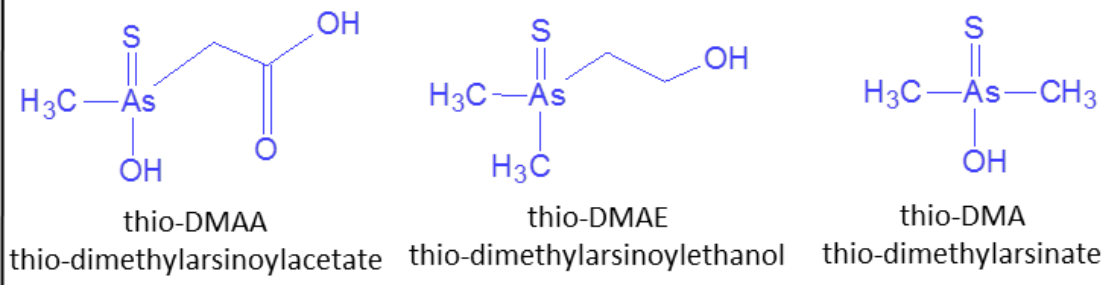 \\
\hline $\begin{array}{l}\text { Dietary } \\
\text { Arsenic }\end{array}$ & 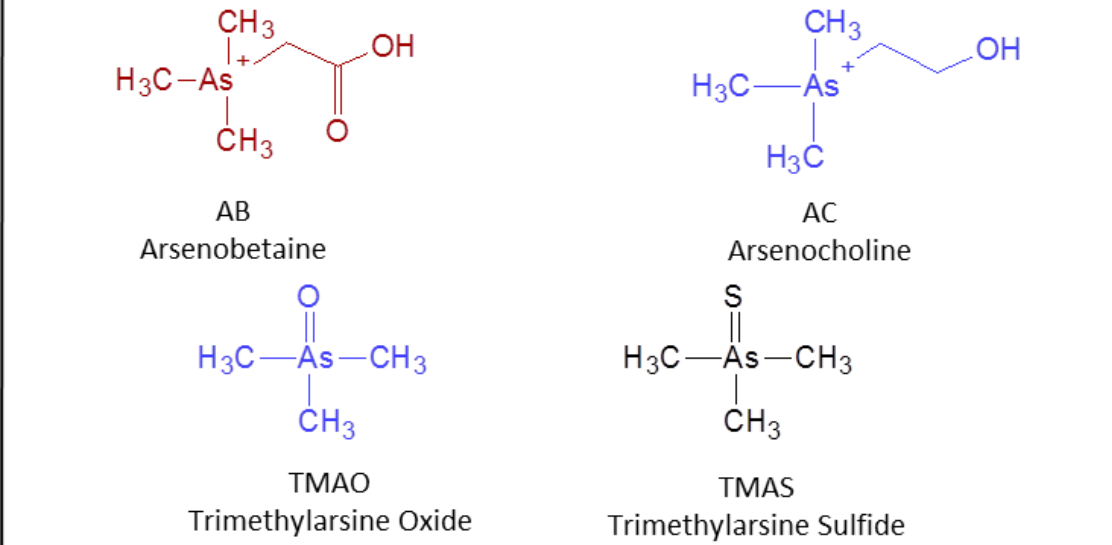 \\
\hline
\end{tabular}

Figure 4 Structures of arsenic compounds relevant to biological monitoringand the work in this thesis. Red structures indicate arsenic compounds analysed for biological monitoring purposes at HSL. Blue structures indicate those investigated in Chapter 3 of this thesis, but are not usually analysed for biological monitoring. 


\subsubsection{Arsenic Compounds - Inorganic Arsenic}

In the environment inorganic arsenic is naturally occurring primarily through volcanic action, but also as a result of industrial processes such as mining, smelting and burning of arsenic rich coal. ${ }^{93}$ Inorganic arsenic exists as the valence states arsenite $(+3$, trivalent: $\left.\mathrm{As}^{3+}\right)$ and arsenate $\left(+5\right.$, pentavalent: $\left.\mathrm{As}^{5+}\right)$. Arsenic trioxide $\left(\mathrm{As}_{2} \mathrm{O}_{3}\right)$, sodium arsenite $\left(\mathrm{NaAsO}_{2}\right)$ and arsenic trichloride $\left(\mathrm{AsCl}_{3}\right)$ are the most common forms of $\mathrm{As}^{3+}$ compounds. ${ }^{100,109}$ Arsenic pentoxide $\left(\mathrm{As}_{2} \mathrm{O}_{5}\right)$, arsenic acid $\left(\mathrm{H}_{3} \mathrm{AsO}_{4}\right)$ and arsenates such as lead arsenate $\left(\mathrm{PbHAsO}_{4}\right)$ and calcium arsenate $\left(\mathrm{Ca}_{3}\left(\mathrm{AsO}_{4}\right)_{2}\right)$ are common $\mathrm{As}^{5+}$ compounds. ${ }^{100,109}$ Trivalent arsenic species are considered more toxic than pentavalent ones as reflected by their $\mathrm{LD}_{50}$ values as shown in Table 3. In humans, after exposure to inorganic arsenic, it is absorbed into the blood with a half-life of $1-2$ hours. ${ }^{15}$ In the event of $\mathrm{As}^{5+}$ exposure, $\mathrm{As}^{5+}$ is reduced to $\mathrm{As}^{3+}$ mainly in the blood and liver. $\mathrm{As}^{3+}$ is then taken up into cells where it is converted to methylated metabolites. The main excretory pathway is in urine in two phases estimated at 20 and 300 hours. $^{15}$

\subsubsection{Arsenic Compounds - Organic Arsenic}

\subsubsection{Human Methylated Metabolites}

Most organisms will metabolise inorganic arsenic to monomethylated (MMA) arsenic species and dimethylated (DMA) arsenic species, which are shown in Figure 4. Only humans excrete significant amounts of the pentavalent monomethylated $\left(\mathrm{MMA}^{5+}\right)$ species, ${ }^{110}$ with the pentavalent dimethylated species $\left(\mathrm{DMA}^{5+}\right)$ being the primary endpoint from inorganic exposure in humans and most mammals. ${ }^{100}$ However, in rats trimethylarsine oxide (TMAO) is found to be the end-point metabolite from inorganic exposure. ${ }^{15}$ The primary site for arsenic methylation is the liver, however studies have shown methylating activity in the testes, kidney and lung tissue. ${ }^{111,112}$ For arsenic methylation to occur the presence of a methyltransferase enzyme such as As3MT is crucial, ${ }^{113,114}$ enabling the transfer of the methyl donor S-adenosylmethionine (SAM) in the presence of glutathione (GSH). ${ }^{115}$ This exact sequence of events has not been fully 
elucidated, however the classical or originally proposed arsenic biotransformation pathway in most mammals including humans, is that after the initial reduction step of $\mathrm{As}^{5+}$ to $\mathrm{As}^{3+}$, it is followed by steps of oxidation methylation and reduction methylation producing $\mathrm{MMA}^{5+}, \mathrm{MMA}^{3+}, \mathrm{DMA}^{5+}$ and finally $\mathrm{DMA}^{3+}$, as shown in Figure $5 .{ }^{116}$

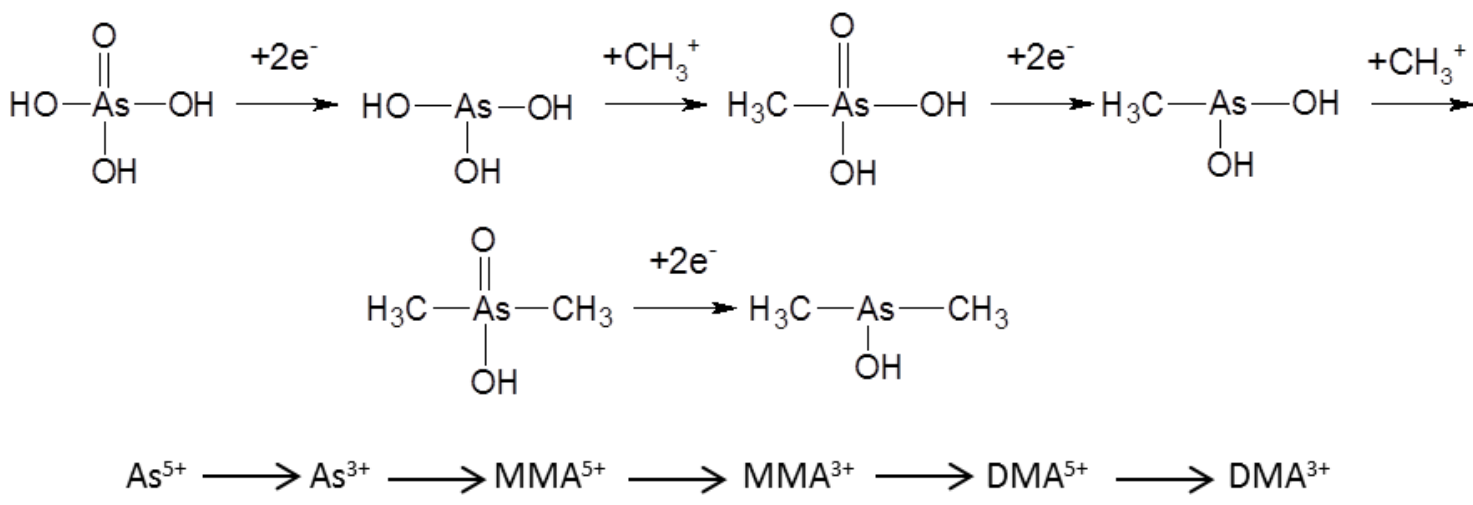

Figure 5 The proposed classical pathway of inorganic arsenic biotransformation of oxidation methylation and reduction methylation steps in humans.

Historically the methylation of arsenic was considered a detoxification pathway until in vitro studies found $\mathrm{MMA}^{3+}$ to be more toxic than inorganic arsenic whilst both $\mathrm{MMA}^{3+}$ and $\mathrm{DMA}^{3+}$ damaged DNA more than $\mathrm{As}^{3+} .{ }^{115,117}$ An in vitro study by Mass et al ${ }^{118}$ supported this viewpoint, determining that both trivalent methylated species caused DNA damage to human lymphocytes. The fact there is a lack of trivalent methylated species found in urine, which contradicted $\mathrm{DMA}^{3+}$ being the end point metabolite ${ }^{119}$ and that both $\mathrm{MMA}^{5+}$ and $\mathrm{DMA}^{5+}$ do not seem to undergo further conversion and are found not to be involved in the oxidative methylation pathway, ${ }^{120}$ has led researchers to investigate biotransformation and propose new pathways. ${ }^{121-123}$ The proposed new pathways, though all slightly different, involve steps of methylation and oxidation to produce $\mathrm{MMA}^{3+}$ and $\mathrm{DMA}^{3+}$ first, followed by $\mathrm{MMA}^{5+}$ and $\mathrm{DMA}^{5+}$ second. In addition, DMA is not metabolised from MMA. The literature scrutinises the existence of trivalent methylated species in human urine due to both their instability and rareity. ${ }^{119}$ Studies by Gong et $a l_{,}^{124}$ Mandel et $a l^{125}$ and Suzuki et $a l^{126}$ have reported $\mathrm{MMA}^{3+}$ and $\mathrm{DMA}^{3+}$ in human urine but subsequently had their sample integrity questioned. ${ }^{119}$ 
Both MMA and DMA have been shown to cause adverse effects to the bladder, kidney, thyroid and gastrointestinal tract in animal studies, with DMA causing carcinogenic effects. ${ }^{109,127}$ However, the lack of conclusive evidence for human toxicity, led to IARC classifying methylated arsenic species of DMA and MMA as Group 2B agents defined as "possibly carcinogenic to humans". ${ }^{31}$ In recent years, the discovery of thiodimethylarsinate (thio-DMA) (as discussed in Chapter 3 of this thesis) has led to further questions of the credibility of trivalent methylated species.

\subsubsection{Non-Human Occurring Metabolites}

Species of arsenic from a dietary source constitute two groups, tetra alkylarsonium compounds such as arsenobetaine (AB), arsenocholine (AC) and trimethylarsine oxide (TMAO) (as shown in Figure 4) and trialkylarsine oxide compounds like arsenosugars (Figure 6). Other additional dietary compounds include thioarsenicals (Figure 4 and Figure 6) and arsenolipids (Figure 6). As indicated by their $\mathrm{LD}_{50}$ values in Table $3, A B$, $A C$ and TMAO are considered to be non-toxic and, as such, IARC has classified $A B$ and organic arsenicals not metabolised by humans as Group 3 agents, defined as "not classifiable as to its carcinogenicity to humans". ${ }^{31}$ They are primarily found in marine organisms such as algae, seaweed, shellfish, squid and fish ${ }^{128,129}$ with $A B$ being the most abundant and wide spread species. Arsenosugars and arsenolipids are also associated with marine organisms but will be discussed further in section 1.3.4.3. The primary source of arsenobetaine is marine algae. ${ }^{128}$ The cell membranes of marine algae have transport systems to take-up essential phosphate from seawater however, when arsenate species are present in water, this transport system cannot differentiate between the two, therefore leading to arsenic within the cell. ${ }^{128}$ The algae produce arsenic species such as $A B$ in addition to methylated species as a way of detoxifying $\mathrm{As}^{5+}$, hence how these non-human arsenic species and their methylated metabolites, in addition to inorganic arsenic, can enter the marine food chain and subsequently humans via their diet. Humans and mammals are unable to produce or metabolise $A B$ or $\mathrm{AC}$ and they are quickly excreted in urine. ${ }^{130}$ The same was thought of TMAO, until several studies demonstrated the presence of TMAO in human urine after the 
ingestion and metabolism of an arsenosugar. ${ }^{131-133}$ Although these dietary species are predominately found in marine life, it is important to note that they are not confined to marine organisms and scientific exploration has found other species capable of metabolising $A B$ from inorganic arsenic, such as earthworms, fungi and birds. ${ }^{134}$

\subsubsection{New Generation of Oxo- and Thioarsenicals}

Approximately 20 arsenosugars have been identified of which Figure 6 shows the four compounds $\left(\mathrm{DMA}^{5+}\right.$-sugar-glycerol, $\mathrm{DMA}^{5+}$-sugar-phosphate, $\mathrm{DMA}^{5+}$-sugar-sulfate and $\mathrm{DMA}^{5+}$-sugar-sulfonate) deemed the most widespread. ${ }^{135}$ Arsenolipids are a group of compounds which include arsenic fatty acids, hydrocarbons and glycophospholipids. ${ }^{135}$ Unlike $A B$ and $A C$, arsenosugars and arsenolipids are metabolised in humans and can be transformed after ingestion. However, like $A B$ and $A C$ they are very much associated with marine organisms and are found in algae, seaweed and fish oils. $^{132,133,136,137}$ Upon ingestion of both arsenosugars and arsenolipids, the major metabolite in urine is DMA. ${ }^{132}$ However a number of DMA analogues called dimethylarsinoyl compounds (where the arsinoyl group represents either $A s=0$ or $A s=S$ ) can also be found in urine. These compounds, termed oxo- and thioarsenicals are shown in Figure 4 and include: dimethylarsenoacetate (DMAA); dimethylarsenoethanol (DMAE); thio-dimethylarsenoacetate (thio-DMAA); thiodimethylarsenoethanol (thio-DMAE); thio-dimethylarsinate thio-DMA (also referred to as dimethylthioarsinic acid (DMTA)). The first study to report an oxoarsenical in human urine after the ingestion of an arsenosugar was Francesconi et $a l^{131}$ in 2002 . This study reported DMAE alongside the first reporting of methylated TMAO, excreted in human urine along with numerous unknowns. The first study to report DMAA was Hansen et $a l^{138}$ in 2003. DMAE, DMAA and the primary metabolite DMA were identified in the urine of sheep located in North Ronaldsway (Scotland), which are unique in the fact they are chronically exposed to arsenosugars due to a diet composed primarily of seaweed. It was the study of these sheep by the same research group which also identified the first thioarsenical ${ }^{136}$ (thio-DMAA) in 2004. Further human dosing studies of an arsenosugar by Francesconi's group ${ }^{132,133}$ resulted in the excretion of 12 arsenic 
compounds including: DMA, TMAO, DMAE, DMAA, thio-DMAA, thio-DMAE, thio-DMA, trimethylarsine sulphide (TMAS), the arsenosugar itself, a thio-arsenosugar and two remaining unknowns, which were an oxo- thioarsenical pair. The first investigation of human metabolism of an arsenolipid was by Schmeisser et $a l^{139}$ and resulted in the excretion of dimethylarsenopropanoic acid (DMAP), thio-dimethylarsenopropanoic acid (thio-DMAP), dimethylarsenobutanoic acid (DMAB) and thiodimethylarsenobutanoic acid (thio-DMAB). Two arsenolipid compounds, along with DMAP, DMAB, thio-DMAP and thio-DMAB are shown in Figure 6.

A limitation of the analysis of these compounds is the lack of commercially available standards, to either aid identification or to quantitatively analyse them. These arsenic compounds will possibly become more prevalent in the human diet and our ability to now detect unidentified arsenic species in human urine (thought primarily to be because of the advancement in analytical technology), is going to become a concern if commercially available standards are not available to help identify and quantify them. Chapter 3 of this thesis outlines the prominence of these arsenic compounds within routine biological monitoring urine samples and the difficulty in identifying them.

\subsubsection{Toxicity of Arsenosugars, Arsenolipids and their Respective Human Metabolites}

This emergence in the identification of numerous new metabolites resulting from the ingestion of arsenosugars and arsenolipids in humans, is a toxicological concern. In addition, the fact that thio-DMA has been reported in the urine of individuals exposed to inorganic arsenic in drinking water ${ }^{140,141}$ raises issues of its carcinogenicity. Of the four common arsenosugars shown in Figure 6, in vitro toxicological studies of only $\mathrm{DMA}^{5+}$-sugar-glycerol and $\mathrm{DMA}^{5+}$-sugar-sulphate have been performed and found both to be neither genotoxic nor cytotoxic. ${ }^{142,143}$ However, in a study of $\mathrm{DMA}^{3+}$-sugarglycerol, although never seen in a biological system, ${ }^{135}$ it was observed to be significantly cytotoxic to cells in comparison to $\mathrm{DMA}^{5+}$ and $\mathrm{MMA}^{5+}$, but less so than $\mathrm{As}^{3+}$ and $\mathrm{As}^{5+} .{ }^{143}$ Additionally, another in vitro study found thio-DMA ${ }^{5+}$-sugar-glycerol exerted low cytotoxicity. ${ }^{144}$ An in vitro study looking at three arsenic containing 
hydrocarbons found them to be as cytotoxic as $\mathrm{As}^{3+}$ in human bladder and liver cells, ${ }^{145}$ however no cytotoxicity was found with arsenolipid metabolites DMAP and thioDMAP. $^{146}$

The DMA analogue, thio-DMA has gained the greatest attention as it has been reported as a product of both inorganic arsenic ${ }^{141}$ and arsenosugar ${ }^{95,132}$ metabolism. All in vitro toxicity tests of thio-DMA, found it to be the same or more toxic than $\mathrm{As}^{3+}$ in human bladder cells, ${ }^{147-149}$ human lung cells ${ }^{150}$ and human skin cells, ${ }^{151}$ with some studies reporting a reduction in cytotoxic effects with GSH depletion and an increase with GSH addition. ${ }^{152,153}$ Naramandura's research group have done much investigation of thioarsenicals in terms of in vitro toxicity studies, animal studies and metabolism determination. A study of human bladder cells found exposure to thio-DMA generated a high level of intracellular reactive oxygen species which was suggested to be the cause of cell death rather than the thio-DMA itself. ${ }^{148}$ They reported that in hamsters, thio-DMA bound to proteins in the same way as trivalent arsenic species, especially $\mathrm{DMA}^{3+}$ and was efficiently taken up into red blood cells (RBC), converted to $\mathrm{DMA}^{5+}$ and distributed around the body to the liver, kidneys and lungs. ${ }^{154}$ However, thio-arsenical dimethylmonothioarsinic acid (dithio-DMA or DMDTA) did not bind to proteins or modify but was excreted unchanged. ${ }^{154,155} \mathrm{~A}$ study of in vitro human RBC also demonstrated that thio-DMA was converted from $\mathrm{DMA}^{3+}$, which was swiftly taken up into RBC and rapidly released after conversion. ${ }^{156}$ 


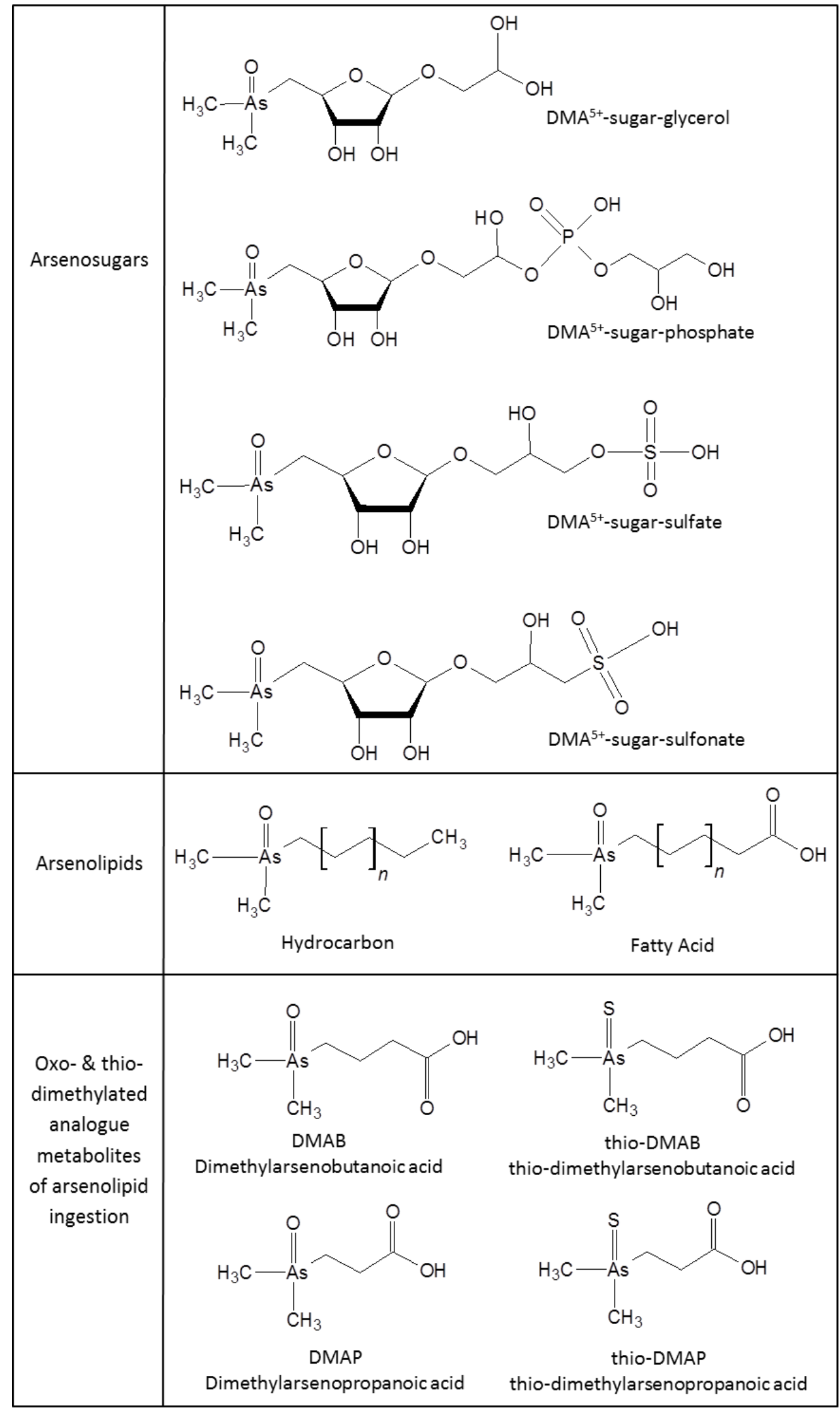

Figure 6 Arsenic compounds known as arsenosugars, arsenolipids and the metabolised dimethylated analogues after arsenolipid ingestion. 


\subsubsection{Dietary Influences}

The metabolism of arsenic compounds varies depending on the specific compound and the exposure route. The metabolism of arsenic species will also influence the different toxicities between these various arsenic compounds and their toxic effects in different animal species. For example, the toxicity of an ingested methylated arsenic compound in human urine from a dietary source will differ from that of a methylated arsenic compound in human urine as a result of inorganic arsenic exposure. ${ }^{15}$ Arsenic is ranked as the $12^{\text {th }}$ most abundant element in the human body ${ }^{117}$ and the vast majority of this exposure in the general population is through diet. As discussed above, $A B, A C$, TMAO, arsenosugars, arsenolipids and other oxo- and thioarsenicals are all dietary species of arsenic. However, inorganic arsenic, $\mathrm{DMA}^{5+}$ and $\mathrm{MMA}^{5+}$ are also found in dietary sources. DMA and sometimes MMA can be found in rice, other plant based foods and seafood. People who eat seafood have increased urinary $D_{M} A^{5+}$ compared to those who do not. ${ }^{157}$ Inorganic arsenic can be found in chicken, ${ }^{109,158}$ rice, ${ }^{158-161}$ cereal, $^{109,162}$ mushrooms, ${ }^{163,164}$ wine and beer, ${ }^{165,166}$ fruit juices ${ }^{166,167}$ and dairy products. ${ }^{109}$ Only trace levels are generally found in seafood and seaweed, ${ }^{130}$ with the exception of brown algae and bivalves such as blue mussels. ${ }^{130,135,137,158}$ Arsenic exists in all natural waters with concentrations ranging from $<0.5$ to $>5000 \mu \mathrm{g} / \mathrm{L}$, ${ }^{168}$ with inorganic arsenic being the predominant species found. ${ }^{168}$ The World Health Organisation (WHO) recommends a maximum value for arsenic in drinking water of 10 $\mu \mathrm{g} / \mathrm{L}^{169}$. Although freshwater normally contains less than this, most frequently less than $1 \mu \mathrm{g} / \mathrm{L},{ }^{170}$ ground water drinking supplies with high levels of naturally present inorganic arsenic occur in Argentina, Bangladesh, Chile, China, Hungary, India, Mexico, the US and Vietnam. ${ }^{168,170,171}$ Elevated levels of arsenic in private drinking water supplies of up to $435 \mu \mathrm{g} / \mathrm{L}$ have also been reported in Cornwall, UK. ${ }^{172,173}$

\subsubsection{Industrial and Occupational Exposure to Arsenic}

Occupational exposure to arsenic compounds occurred as far back as 5000 years ago. The melting of a glacier caused the frozen body of a man to be discovered in 1991 by two German tourists in the Italian Alps. ${ }^{174}$ The ice, which had helped to preserve the 
man's body and his belongings, enabled scientists to take biological samples for analytical investigation. In addition to dating the body as being 5300 years old, analysis of the iceman's hair revealed high levels of arsenic and copper. An axe was found amongst his possessions composed of $99.7 \%$ copper; the elevated arsenic and copper results have led scientists to believe he may have been involved in copper smelting. ${ }^{174}$

Occupational exposure to inorganic arsenic can occur in many industries and occupations, as outlined in Table 4.

Table 4 Industries and occupations where exposure to inorganic arsenic occurs.

\begin{tabular}{|c|c|c|}
\hline \multicolumn{3}{|c|}{ Industries \& Occupations ${ }^{109}$} \\
\hline $\begin{array}{l}\text { Semi-conductor } \\
\text { Manufacture }\end{array}$ & Glass Production & Microelectronics \\
\hline Wood Preservation & $\begin{array}{c}\text { Burning/Recycling of } \\
\text { Preserved Wood }\end{array}$ & $\begin{array}{c}\text { Pesticide Manufacture or } \\
\text { Use }\end{array}$ \\
\hline $\begin{array}{c}\text { Burning of Arsenic Rich } \\
\text { Coal }\end{array}$ & Refining Metal Ore & Non-ferrous Smelting \\
\hline Copper Smelting & Alloy Manufacture & $\begin{array}{c}\text { Assembly/Recycling of } \\
\text { Lead Acid Batteries }\end{array}$ \\
\hline Pyrotechnics & Pigments \& Dyes & Electrophotography \\
\hline
\end{tabular}

Occupational inhalation of airborne arsenic is in the form of particulate matter that deposits in the lungs, followed by absorption. ${ }^{93}$ Little is known about the deposition and absorption of inhaled arsenic in humans but it is thought that the form of inorganic arsenic is a fundamental factor. ${ }^{93}$ Inhaled insoluble $\mathrm{As}^{5+}$ is thought to be absorbed at a much lower rate than $\mathrm{As}^{3+}$. A study by Yager et $\left.a\right|^{175}$ correlated arsenic air measurements with arsenic speciated urinary measurements of boiler maintenance workers in coal-fired power plants in the US. The workers were exposed to arsenic in the form of calcium arsenate. Yager et $a l^{175}$ noted that air measurements for a 8-hour time weighted average of $10 \mu \mathrm{g} / \mathrm{m}^{3}$, resulted in a mean sum of inorganic arsenic and methylated metabolites in urine a third lower than in copper smelters who were 
exposed to arsenic trioxide and workers at a arsenic trioxide refinery. However, a study by Morton \& Mason ${ }^{157}$ found that arsenate exposed timber treatment workers had significantly higher urinary levels of inorganic arsenic and methylated metabolites than arsenite exposed semi-conductor workers. This difference was judged to be as a result of higher levels of exposure, different routes of exposure and poor worker behaviour concerning PPE for the arsenate wood preservation workers compared to improved engineering controls and PPE of the semi-conductor workers. ${ }^{157}$ In the 1950s, a study involving the inhalation of sodium arsenite in eight volunteers (some of whom had lung cancer) found the deposition of arsenic particulate in the lungs to be approximately $40 \%$, of which $75-85 \%$ of this amount was absorbed. This indicated that the overall absorption, expressed as a percentage of inhaled arsenic was a total of 30$34 \%$ of the initial inhaled dose. ${ }^{98}$ However, reported results based on individuals with ill health must be viewed with caution, as this may not be true of people without lung disease. Smelters exposed to arsenic trioxide dusts excreted approximately $40-60 \%$ of the inhaled arsenic in their urine. ${ }^{176,177}$ Those with the highest urinary arsenic levels showed a higher excretionary rate of the absorbed arsenic compared to those with the lowest urinary arsenic values. ${ }^{177}$

When arsenic is ingested more than $90 \%$ of the dissolved dose is absorbed from the gastrointestinal tract regardless of whether is it $\mathrm{As}^{3+}$ or $A s^{5+} \cdot{ }^{93}$ It is also likely that a proportion of the inhaled particles of inorganic arsenic in the form of dusts will be swallowed and absorbed from the gastrointestinal tract. ${ }^{98}$

\subsubsection{Biological Monitoring of Arsenic Exposure}

After exposure to inorganic arsenic, it is quickly distributed throughout the body and rapidly cleared from the blood in humans. ${ }^{28}$ This rapid clearance of arsenic from blood makes it an unsuitable matrix for biological monitoring but it would be suitable for investigating very recent and high level exposure, ${ }^{93}$ such as an accidental poisoning. The main route of excretion, after either inorganic or organic arsenic exposure, is via urine. $A s \mathrm{As}^{3+}$ binds to proteins, excretion after exposure to $\mathrm{As}^{5+}$ tends to be faster than $\mathrm{As}^{3+} .{ }^{28}$ Regardless of the extent of exposure, the distribution of arsenic in urine of 
various population groups is reasonably constant with $10-30 \%$ inorganic arsenic, 10 $20 \%$ MMA and $60-70 \%$ DMA. $^{28}$ Though it has been suggested that a higher proportion of MMA in urine indicates an individual's poor methylation capacity. ${ }^{137}$

Total arsenic analysis is now considered completely inappropriate due to the inability to distinguish between non-toxic $A B$ and toxic inorganic species. Speciation methods often have long sample acquisition times making them unsuitable for routine analysis, therefore the analytical method of choice for many laboratories is hydride generation. Hydride generation eliminates dietary $A B$ and $A C$ due to them not forming a hydride, but instead through reduction and hydride generation gives a total combined value of the inorganic arsenic species and the methylated metabolites, DMA and MMA. Unfortunately, the increased incidence of DMA and MMA in food and drink has resulted in hydride generation being deemed no longer fit for purpose due to the inability to distinguish methylated metabolites of dietary exposure from methylated metabolites of inorganic arsenic exposure. Furthermore, it is known that the technique of hydride generation struggled to convert $100 \%$ of the DMA present in a urine sample to $\mathrm{As}^{3+}$ to form a hydride. The only way to accurately determine the type of exposure and distribution of arsenic species in a urine sample is to perform speciation analysis. A difficultly with this approach is that there are no background reference values for any of the five species of arsenic. Chapter 2 of this thesis attempts to rectify this issue by demonstrating a routine fast arsenic speciation method and reporting a mean and $90^{\text {th }}$ percentile values for five species of arsenic in a background occupationally unexposed population. Another problem is the lack of BMGV for any of the five species of arsenic, however there is the U.S. BEI of $35 \mu \mathrm{g} / \mathrm{L}$ (approximately $40 \mu \mathrm{mol} / \mathrm{mol}$ creatinine) and the German BAT of $50 \mu \mathrm{g} / \mathrm{L}$ (approximately $55 \mu \mathrm{mol} / \mathrm{mol}$ creatinine); both are based on the sum of inorganic species and methylated species. 


\subsection{Chromium}

\subsubsection{Physical Properties}

Chromium $(\mathrm{Cr})$ is the first metal of group 6 in the periodic table. Chromium has the atomic number 24 and a mass of 51.996 amu. It has four stable isotopes of which ${ }^{52} \mathrm{Cr}$ (83.76\%) is the most abundant, ${ }^{50} \mathrm{Cr}(4.31 \%),{ }^{53} \mathrm{Cr}(9.55 \%)$ and ${ }^{54} \mathrm{Cr}(2.38 \%)$ being the other three. Chromium is ubiquitous and classed as the $24^{\text {th }}$ most naturally occurring element ${ }^{178}$ found in rock, soil, plants, animals and volcanic dusts, however not naturally in air.

Chromium is a transition element that exhibits nine valence states from -2 to +6 . However only +3 and +6 are environmentally and thermodynamically stable and are considered the only species of interest in relation to human exposure. Hexavalent chromium exists in the valence state $+6 / \mathrm{Cr}(\mathrm{VI})$ such as chromate $\left(\mathrm{CrO}_{4}{ }^{2-}\right)$ or dichromate $\left(\mathrm{Cr}_{2} \mathrm{O}_{7}{ }^{2-}\right)$ and is toxic. Trivalent chromium exists in the valence state $+3 / \mathrm{Cr}(\mathrm{III})\left(\mathrm{CrCl}_{3}\right.$ and $\left.\mathrm{Cr}_{2} \mathrm{O}_{3}\right)$ and is deemed an essential element in humans, needed for the metabolism of sugar, protein and fat. ${ }^{179}$

The stability of both species of chromium is heavily dependent on $\mathrm{pH}$, with trivalent chromium being stable under acidic conditions and hexavalent chromium under more alkaline conditions. Stability and pH conditions of both chromium species are discussed further in Chapter 4 of this thesis.

\subsubsection{Chromium Toxicity and Effects on Human Health}

Chromium toxicity is dependent on its species, with $\mathrm{Cr}(\mathrm{VI})$ being considerably more toxic than $\mathrm{Cr}(\mathrm{III})$. After exposure to $\mathrm{Cr}(\mathrm{VI})$, it is naturally reduced to $\mathrm{Cr}(\mathrm{III})$ by biological reduction processes. Although $\mathrm{Cr}(\mathrm{III})$ is an essential element, dermal exposure can still result in health effects such as skin irritation and allergic contact dermatitis, ${ }^{180}$ however due to its poor solubility it is far less efficient at producing an allergic 
response than $\mathrm{Cr}(\mathrm{VI}) .{ }^{180} \mathrm{Cr}(\mathrm{III})$ is not considered carcinogenic, ${ }^{28}$ with IARC classifying it as a Group 3 agent, defined as "not classifiable as to its carcinogenicity to humans". ${ }^{31}$

Hexavalent chromium however is considered to be carcinogen in addition to being a mutagen, asthmagen and inducer of skin dermatitis. ${ }^{178}$ It is rarely found naturally in the environment only occurring as the mineral crocoite $\left(\mathrm{PbCrO}_{4}\right),{ }^{181}$ therefore most environmental $\mathrm{Cr}(\mathrm{VI})$ existence is normally as a result of industrial processes and manmade production. IARC has classified $\mathrm{Cr}(\mathrm{VI})$ as a Group 1 agent, defined as "carcinogenic to humans". ${ }^{31}$ Compounds of $\mathrm{Cr}(\mathrm{VI})$ can be soluble or insoluble which is also linked to toxicity. Water soluble $\mathrm{Cr}(\mathrm{VI})$ compounds, such as sodium chromate $\left(\mathrm{NaCrO}_{4}\right)$, sodium dichromate $\left(\mathrm{Na}_{2} \mathrm{Cr}_{2} \mathrm{O}_{7}\right)$, potassium chromate $\left(\mathrm{K}_{2} \mathrm{CrO}_{4}\right)$ and potassium dichromate $\left(\mathrm{K}_{2} \mathrm{Cr}_{2} \mathrm{O}_{7}\right)$, are more toxic than less soluble $\mathrm{Cr}(\mathrm{VI})$ compounds such as barium chromate $\left(\mathrm{BaCrO}_{4}\right)$ and lead chromate $\left(\mathrm{PbCrO}_{4}\right)^{28,109}$ The health effects associated with $\mathrm{Cr}(\mathrm{VI})$ exposure are vast, with the route of exposure resulting in different toxicity endpoints. Acute ingestion of $\mathrm{Cr}(\mathrm{VI})$ can cause gastroenteritis, gastrointestinal haemorrhage and renal failure. ${ }^{181}$ Skin contact with $\mathrm{Cr}(\mathrm{VI})$ compounds can cause dermal irritation and allergic contact dermatitis, ulceration and burns to the skin. ${ }^{16,181}$ Inhalation exposures can result in respiratory effects such as ulceration of the nasal mucosa, perforation of the nasal septum, bronchitis, occupational asthma, pneumonia and cancers such as nasal, sinus and lung, in addition to other health effects such as a weakened immune system and/or kidney and liver damage. ${ }^{16,179}$

As demonstrated in Table 5, the $\mathrm{LD}_{50}$ of $\mathrm{Cr}(\mathrm{VI})$ for an oral ingested dose is compound dependent. There are several suggestions in the literature estimating the human lethal dose to $\mathrm{Cr}(\mathrm{VI})$ of from 1 to $3 \mathrm{~g}$, ${ }^{181}$ between 50 to $70 \mathrm{mg} / \mathrm{kg}$ body weight for soluble chromate $^{17}$ and 0.5 to $1.0 \mathrm{~g}$ for potassium dichromate. ${ }^{182}$ However, concentrations of $\mathrm{Cr}(\mathrm{VI})>200 \mathrm{mg} / \mathrm{L}$ which are non-fatal to humans can result in gastritis, nephrotoxicity and hepatotoxicity. ${ }^{183}$ It is thought that due to limited information and conflicting studies there is insufficient evidence to suggest that $\mathrm{Cr}(\mathrm{VI})$ is carcinogenic to humans when exposure is via ingestion. ${ }^{183,184}$ This is thought to be due to it being reduced to $\mathrm{Cr}(\mathrm{III})$ by the gastrointestinal system prior to absorption. ${ }^{28}$ However accidental or suicidal ingestions of extremely high doses (for example, a 44-year-old male ingested 
an estimated $4.1 \mathrm{mg} / \mathrm{kg}$ chromic acid, ${ }^{185}$ a 33-year-old male ingested $125 \mathrm{~mL}$ of chromic acid ${ }^{186}$ and a 17 -year-old male ingested $5 \mathrm{~g}$ of potassium dichromate ${ }^{182}$ ) have resulted in fatalities. Conversely, there has been a report of a 58-year-old male surviving an ingestion of $30 \mathrm{~g} / \mathrm{L}$ (estimated $3 \mathrm{~g}$ ) of potassium dichromate and being discharged with no renal or liver failure. ${ }^{187}$ Table 5 also shows there is a stark difference between the $\mathrm{LD}_{50}$ value for male and female rats, with females showing a much a greater sensitivity to the toxicity of $\mathrm{Cr}(\mathrm{VI})$ compounds than males.

Table $5 \mathrm{LD}_{50}$ values of chromium compounds in laboratory experiments

\begin{tabular}{|c|c|c|c|c|}
\hline Species & Chromium Compound & Test subject & $\begin{array}{c}\mathrm{LD}_{50} \\
(\mathrm{mg} / \mathrm{kg})\end{array}$ & Ref \\
\hline $\mathrm{Cr}(\mathrm{VI})$ & Strontium chromate $\left(\mathrm{SrCrO}_{4}\right)$ & Male rats & 811 & 16 \\
\hline $\mathrm{Cr}(\mathrm{VI})$ & Chromium trioxide $\mathrm{CrO}_{3}$ & $\begin{array}{c}\text { Female Rats } \\
\text { Male Rats }\end{array}$ & $\begin{array}{l}25 \\
29\end{array}$ & 16 \\
\hline $\mathrm{Cr}(\mathrm{VI})$ & Sodium chromate $\left(\mathrm{Na}_{2} \mathrm{CrO}_{4}\right)$ & $\begin{array}{c}\text { Female Rats } \\
\text { Male Rats }\end{array}$ & $\begin{array}{l}13 \\
28\end{array}$ & 188 \\
\hline $\mathrm{Cr}(\mathrm{VI})$ & Sodium dichromate $\left(\mathrm{Na}_{2} \mathrm{Cr}_{2} \mathrm{O}_{7}\right)$ & $\begin{array}{c}\text { Female Rats } \\
\text { Male Rats }\end{array}$ & $\begin{array}{l}14 \\
21\end{array}$ & 188 \\
\hline $\mathrm{Cr}(\mathrm{VI})$ & Potassium dichromate $\left(\mathrm{K}_{2} \mathrm{Cr}_{2} \mathrm{O}_{7}\right)$ & $\begin{array}{c}\text { Female Rats } \\
\text { Male Rats }\end{array}$ & $\begin{array}{l}17 \\
26\end{array}$ & 188 \\
\hline $\mathrm{Cr}(\mathrm{VI})$ & Calcium chromate $\left(\mathrm{CaCrO}_{4}\right)$ & $\begin{array}{c}\text { Female Rats } \\
\text { Male Rats }\end{array}$ & $\begin{array}{l}108 \\
249\end{array}$ & 189 \\
\hline $\mathrm{Cr}(\mathrm{VI})$ & Ammonium dichromate $\left(\left(\mathrm{NH}_{4}\right)_{2} \mathrm{Cr}_{2} \mathrm{O}_{7}\right)$ & $\begin{array}{c}\text { Female Rats } \\
\text { Male Rats }\end{array}$ & $\begin{array}{l}19 \\
22\end{array}$ & 188 \\
\hline $\operatorname{Cr}(I I I)$ & Chromium acetate $\mathrm{Cr}\left(\mathrm{CH}_{3} \mathrm{COO}\right)_{3}$ & Rats & 2365 & 16 \\
\hline $\operatorname{Cr}(I I I)$ & Chromium nitrate $\mathrm{Cr}\left(\mathrm{NO}_{3}\right)_{3}$ & $\begin{array}{c}\text { Female Rats } \\
\text { Male Rats }\end{array}$ & $\begin{array}{l}183 \\
200\end{array}$ & 189 \\
\hline
\end{tabular}

The toxicity and especially genotoxicity of $\operatorname{Cr}(\mathrm{VI})$ is linked to its ability to cross cell membranes and although it has been shown to be unreactive towards DNA, ${ }^{184}$ it has the ability to undergo intracellular reduction which produces the reactive chromium species and oxygen radicals which cause the DNA damage. ${ }^{28}$ Ascorbate with glutathione are the principal reducers of $\mathrm{Cr}(\mathrm{VI})^{190,191}$ however, cysteine, hydrogen 
peroxide and mitochondrial enzymes have all been found to aid the reduction of $\mathrm{Cr}(\mathrm{VI})$ to $\mathrm{Cr}(\mathrm{V}), \mathrm{Cr}(\mathrm{IV})$ and subsequently $\mathrm{Cr}(\mathrm{III}) .{ }^{28}$ During this process reactive hydroxyl radicals and reactive oxygen species (ROS) are generated ${ }^{184}$ leading to oxidative stress and damage to DNA, affecting DNA replication. ${ }^{28}$ The reason that $\mathrm{Cr}(\mathrm{III})$ is considerably less toxic is because of its poor ability to permeate cell membranes, therefore $\mathrm{Cr}(\mathrm{VI})$, which is reduced to $\mathrm{Cr}(\mathrm{III})$ prior to cell absorption, has its genotoxic abilities supressed. Although $\mathrm{Cr}(\mathrm{III})$ can be oxidised to $\mathrm{Cr}(\mathrm{VI})$ in alkaline conditions, there is no evidence that biological systems can convert $\mathrm{Cr}(\mathrm{III})$ to $\mathrm{Cr}(\mathrm{VI}) .{ }^{181,184}$

\subsubsection{Industrial and Occupational Exposure to Chromium}

In an occupational setting exposure to chromium is primarily through inhalation, however, skin absorption and ingestion are also exposure pathways. Chromium is a multi-functional metal that has the ability to provide alloys and materials with added strength, hardness and resistance to temperature, in addition to wear and corrosion resistance. ${ }^{192,193}$ For example, chromium is used to harden steel during the manufacture of stainless steel, chromium plating provides a hard shiny coating to other metals and plastics to restrict corrosion and provide an enhanced appearance and chromium is added to paints and pigments for its corrosive resistant abilities in extreme conditions such as those required in the maritime and aeronautic industries. ${ }^{193,194}$ Table 6 outlines many of the industries, occupations and activities where exposure to $\mathrm{Cr}(\mathrm{VI})$ occurs.

Table 6 Industries and occupations where exposure to hexavalent chromium occurs.

\section{Industries \& Occupations Involved with the Production or Use of Chromium Containing Metals and Goods

Production

Chromates

Stainless Steel

Chrome Paints

Chrome Pigment \& Dye

Chromium Alloys 


\section{Industries \& Occupations Involved with the Production \\ or Use of Chromium Containing Metals and Goods 33,179,195}

\section{Industries}

Chrome plating Leather tanning Steel mills

Foundries Wood preservation Construction

Plasma Cutting Pulp production

Industrial processes

Welding Grinding Polishing

Pesticides Paint spraying / removal Smelting

The industries with the highest exposure to $\operatorname{Cr}(\mathrm{VI})$ compounds include chromate production, ferrochrome and chrome pigment production, chrome plating and stainless steel welding. ${ }^{16}$ Therefore it might not be surprising that there is an increased risk of occupational cancer amongst several of these types of industries and notably amongst chromate production workers, surface engineering chrome-platers and pigment production workers. ${ }^{183,195,196}$ Other health effects associated with inhalation exposure such as respiratory irritations have also been reported at concentrations as low as $2 \mu \mathrm{g} / \mathrm{m}^{3}$ in the chrome plating industry. ${ }^{183}$ In contrast to the $\mathrm{LD}_{50}$ of an oral ingested dose in rats (see Table 5), strontium chromate and zinc chromate due to their solubility are associated with the greatest risk of developing lung cancer. ${ }^{181}$ Table 7 shows the different industrial uses of specific chromium compounds.

Table 7 The purpose and use of specific compounds of hexavalent and trivalent chromium in different industries.

\begin{tabular}{|c|c|c|}
\hline $\begin{array}{c}\text { Hexavalent Chromium } \\
\text { Compound }\end{array}$ & \multicolumn{2}{|c|}{ Industrial Uses ${ }^{109,195}$} \\
\hline \multirow{2}{*}{ Ammonium dichromate } & Leather Tanning & Textile pigments \& dyes \\
\hline \multirow{2}{*}{ Barium chromate } & Corrosion inhibitors & $\begin{array}{c}\text { Paint, Ink \& Plastic pigments } \\
\text { \& dyes }\end{array}$ \\
\cline { 2 - 3 } & \multicolumn{2}{|c|}{ Pyrotechnics } \\
\hline
\end{tabular}




\begin{tabular}{|c|c|c|}
\hline $\begin{array}{l}\text { Hexavalent Chromium } \\
\text { Compound }\end{array}$ & \multicolumn{2}{|c|}{ Industrial Uses ${ }^{109,195}$} \\
\hline Calcium chromate & Corrosion inhibitors & $\begin{array}{c}\text { Paint, Ink \& Plastic pigments } \\
\text { \& dyes }\end{array}$ \\
\hline \multirow{2}{*}{ Chromic trioxide } & Corrosion inhibitors & Metal finishing \\
\hline & \multicolumn{2}{|c|}{ Wood Preservation } \\
\hline \multirow[t]{2}{*}{ Lead chromate } & $\begin{array}{c}\text { Paint, Ink \& Plastic pigments } \\
\text { \& dyes }\end{array}$ & $\begin{array}{l}\text { Chrome yellow paint } \\
\text { (automotive finishes) }\end{array}$ \\
\hline & \multicolumn{2}{|c|}{ Synthetic resins } \\
\hline Potassium chromate & \multicolumn{2}{|c|}{ Textile pigments \& dyes } \\
\hline Potassium dichromate & \multicolumn{2}{|c|}{ Paint, Ink \& Plastic pigments \& dyes } \\
\hline \multirow{3}{*}{ Sodium chromate } & Textile pigments \& dyes & $\begin{array}{c}\text { Paint, Ink \& Plastic pigments } \\
\text { \& dyes }\end{array}$ \\
\hline & Wood Preservation & Corrosion inhibition \\
\hline & Leather tanning & Catalysts \\
\hline \multirow{2}{*}{ Sodium dichromate } & $\begin{array}{c}\text { Manufacture of chromium } \\
\text { chemicals }\end{array}$ & Leather tanning \\
\hline & \multicolumn{2}{|c|}{ Textile pigments $\&$ dyes } \\
\hline Strontium chromate & \multicolumn{2}{|c|}{ Metal finishing } \\
\hline Zinc chromate & $\begin{array}{c}\text { Paint, Ink \& Plastic pigments } \\
\text { \& dyes }\end{array}$ & Metal coating primer \\
\hline $\begin{array}{l}\text { Trivalent Chromium } \\
\text { Compounds }\end{array}$ & \multicolumn{2}{|c|}{ Industrial Uses ${ }^{195}$} \\
\hline Chromium acetate & Emulsion Hardener & $\begin{array}{c}\text { Paint, Ink \& Plastic pigments } \\
\qquad \text { dyes }\end{array}$ \\
\hline Chromium nitrate & Corrosion inhibitor & Textile pigments \& dyes \\
\hline \multirow{2}{*}{ Chromic Sulfate } & Surface Engineering & Ceramic Glazes \\
\hline & \multicolumn{2}{|c|}{ Textile pigments \& dyes } \\
\hline
\end{tabular}




\subsubsection{Biological Monitoring of Chromium Exposure}

No matter the route of exposure, $\mathrm{Cr}(\mathrm{VI})$ is eventually reduced by all biological fluids to $\mathrm{Cr}(\mathrm{III})$. It is possible to measure chromium exposure in urine, red blood cells (RBC) and plasma. However, the measurement of urine and plasma samples results in the inability to distinguish between $\mathrm{Cr}(\mathrm{VI})$ and $\mathrm{Cr}(\mathrm{III})$. The measurement of $\mathrm{RBC}$ is indicative of $\mathrm{Cr}(\mathrm{VI})$ exposure only, (measured as $\mathrm{Cr}(\mathrm{III})$, due to $\mathrm{Cr}(\mathrm{VI})$ cell permeation) however it has been shown that some $\mathrm{Cr}(\mathrm{VI})$ will also be reduced in the plasma, limiting RBC penetration. ${ }^{192}$ In addition, chromium concentration within the RBC will remain elevated for the life of the cell. ${ }^{192}$ Due to its invasive nature and the need for trained medical staff, neither employers or employees favour providing a blood sample for biological monitoring of $\mathrm{Cr}(\mathrm{VI})$ exposure assessment. Therefore, a urine sample is widely accepted and, although this is not an ideal approach to determining $\mathrm{Cr}(\mathrm{VI})$ exposure, it is a practical one and is widely used. ${ }^{192}$ Furthermore, for interpretation of results there are several BMGVs for chromium in urine. Virtually all food contains chromium in the trivalent form ${ }^{193}$ and a background unexposed reference range based on the $95^{\text {th }}$ percentile has been established for a UK population of $<2.9 \mu \mathrm{mol} / \mathrm{mol}$ creatinine. $^{26}$ Because trivalent chromium is very poorly absorbed from the gastrointestinal tract, approximately only $1 \%$ of an ingested oral dose is excreted in urine. ${ }^{193}$ This helps to eliminate the high variability in the dietary contribution of chromium from urinary total chromium measurements, which is very common with total arsenic analysis and dietary arsenic. The current chromium HSE BMGV ${ }^{24}$ of 10 $\mu \mathrm{mol} / \mathrm{mol}$ of creatinine is based on the $90^{\text {th }}$ percentile of data from companies deemed to have good occupational practice to control exposure. The BEI for total chromium based on urine collected at the end of shift at the end of the working week is $25 \mu \mathrm{g} / \mathrm{L}$ $(40 \mu \mathrm{mol} / \mathrm{mol} \text { creatinine })^{22}$

Urinary chromium levels can reflect both past and recent exposure to $\operatorname{Cr}(\mathrm{VI})$, with daily accumulation occurring through the working week of the occupationally exposed, ${ }^{192}$ therefore it is suggested that urine samples are taken towards the end of the working shift towards the end of the working week. The absorption and excretion of $\operatorname{Cr}(\mathrm{VI})$ in the urine is dependent primarily on the type of $\operatorname{Cr}(\mathrm{VI})$ compound and its solubility but 
also the route of exposure, the level of exposure, the dose, body burden and the length of exposure. Studies in welders have suggested the excretion of chromium in urine after exposure by inhalation as a two or three stage process with elimination half-lives of 7 hours, 15-30 days and 3-5 years. ${ }^{16,17}$ Studies in chrome platers have shown a two-stage process with half-lives of 2-3 days followed by 1 month. ${ }^{197}$ One study investigating chromium levels in a 44-year-old plasma cutter found the chromium body burden so high after many years of exposure that the half-life of chromium in urine was 129 months after exposure ceased. ${ }^{198}$

As the primary route of exposure to $\mathrm{Cr}(\mathrm{VI})$ is by inhalation ${ }^{192,199}$ an alternative biological monitoring matrix to help further understand $\mathrm{Cr}(\mathrm{VI})$ inhalation exposures and how $\mathrm{Cr}(\mathrm{VI})$ resides and behaves in the lungs and is subsequently excreted might be EBC. Chapter 4 of this thesis describes the first publication to report a speciation method to simultaneously separate both $\mathrm{Cr}(\mathrm{III})$ and $\mathrm{Cr}(\mathrm{VI})$ in $\mathrm{EBC}$ samples using micro liquid chromatography coupled to inductively coupled plasma mass spectrometry ( $\mu$ LCICP-MS). In addition, Chapter 5 of this thesis describes the first published investigation of both $\mathrm{Cr}(\mathrm{III})$ and $\mathrm{Cr}(\mathrm{VI})$ levels in EBC samples of occupationally exposed individuals and a control group. Both of these studies are the initial investigations into understanding $\mathrm{EBC}$ in relation to the inhalation of $\mathrm{Cr}(\mathrm{VI})$ compounds. 


\subsection{Silicon and Silica}

\subsubsection{Physical Properties}

Silicon $(\mathrm{Si})$ is a metalloid in group 14 of the periodic table. Silicon has the atomic number 14 and a mass of 28.086 amu. It has three stable isotopes of which ${ }^{28} \mathrm{Si}$ $(92.23 \%)$ is the most abundant, the other two being ${ }^{29} \mathrm{Si}(4.67 \%)$ and ${ }^{30} \mathrm{Si}(3.10 \%)$. Silicon is classed as the 2nd most abundant element in the Earth's crust being found in most of the world's rocks (igneous, sedimentary and metamorphic), sands and soils, in addition to water and plants.

Silicon, like carbon, has four electrons in its outer orbital and although it exhibits eight valence states, only -4 and +4 are stable. Silicon itself is considered harmless and is rarely found naturally in the environment; its strong affinity with oxygen ensures most silicon is in the form of silica $\left(\mathrm{SiO}_{2}\right)$ or in the form of silicates.

As shown in Table 8, silica can be found in two forms: crystalline and amorphous.

Table 8 The classification of silica forms ${ }^{200}$

\begin{tabular}{|c|c|c|c|}
\hline \multirow{3}{*}{ Silica Form } & Origin & \multicolumn{2}{|c|}{ Classification } \\
\hline \multirow{4}{*}{ Crystalline } & Natural & $\begin{array}{c}\alpha \text {-quartz } \\
\alpha \text {-tridymite } \\
\alpha \text {-cristobalite } \\
\text { Coesite } \\
\text { Moganite }\end{array}$ & $\begin{array}{c}\beta \text {-quartz } \\
\beta \text {-tridymite } \\
\beta \text {-cristobalite } \\
\text { Stishovite }\end{array}$ \\
\cline { 2 - 4 } & Synthetic & $\begin{array}{c}\text { Keatite } \\
\text { Porosils }\end{array}$ & Silica W \\
\hline \multirow{4}{*}{ Amorphous } & Natural & $\begin{array}{c}\text { Opal } \\
\text { Diatomaceous earths } \\
\text { Vitreous silica }\end{array}$ & $\begin{array}{c}\text { Biogenic silica } \\
\text { Silica fibres }\end{array}$ \\
\cline { 2 - 4 } & Synthetic & $\begin{array}{c}\text { Fused silica } \\
\text { Precipitated silica } \\
\text { Silica gel }\end{array}$ & Fumed silica \\
& & Colloidal silica \\
\hline
\end{tabular}


The difference between crystalline and amorphous silica relates to the particle structure. ${ }^{200}$ Silicon-oxygen tetrahedra $\left(\mathrm{SiO}_{4}\right)$ make up all forms of crystalline and amorphous silica (with the exception of stishovite). ${ }^{200}$ Each silicon-oxygen tetrahedron consists of a silicon atom surrounded by four oxygen atoms; each oxygen atom is shared by two tetrahedra. ${ }^{200}$ The difference is that the tetrahedra of crystalline silica produce a regular three-dimensional framework, which shares each corner with other tetrahedra. The pattern of the crystal structures created are specific to the type of crystalline silica. ${ }^{200}$ Whereas, the tetrahedra in amorphous silica are not regular and the orientation is random which does not result in a crystal structure. ${ }^{200}$

$\alpha$-Quartz is the thermodynamically stable form of crystalline silica (other forms exist in a metastable state) and as such makes up the majority of natural crystalline silica. ${ }^{109}$ Commercial silica is obtained from this naturally occurring source, of which sand and gravel, quartz crystals and diatomites make up the three major commercial products. Rocks and stones composed of crystalline silica include agate, flint, jasper, sandstone, and chert. ${ }^{109}$ Cristobalite is a natural constituent of volcanic lava and is produced when either quartz or amorphous silica are subject to very high temperatures. ${ }^{201}$ Cristobalite is also formed in the manufacture of silica bricks that are used in industrial furnaces.

\subsubsection{Silica Toxicity and Effects of Human Health}

Amorphous silica is considered non-toxic however crystalline silica has been classified as a group 1 agent by IARC, defined as "carcinogenic to humans" but only when it is inhaled in its quartz or cristobalite forms. ${ }^{31}$ In addition, both the National Toxicology Program (NTP) and the American Conference on Governmental Industrial Hygienists (ACGIH) label crystalline silica as a human carcinogen ${ }^{97}$ and suspected human carcinogen, respectively. Amorphous silica is classified as a group 3 agent by IARC, defined as "not classifiable as to its carcinogenicity to humans". ${ }^{31}$

Silica as a whole is the most abundant dietary element in humans after iron and zinc. $^{202}$ Its health benefits include the reduced risk of cardiovascular disease and alopecia along with strengthening of bones, skin and blood vessels. ${ }^{202}$ Humans can be 
exposed to silica from a variety of sources including cosmetics (lipsticks and powders), toiletries (toothpaste), creams (hand and nail) and detergents in addition to cosmetic and medical implants. However the greatest source of exposure is from diet, with an estimated intake of between $20-50 \mathrm{mg}$ silica per day in Western populations. ${ }^{203,204}$ This concentration of dietary intake is more than twice that of a typical dietary intake of zinc and iron. ${ }^{205}$ As silica is taken up and accumulated in plants for structural purposes, it makes plant based foods (especially cereals) ${ }^{202}$ a high contributor of silica in the diet. ${ }^{202,205}$ Beer is said to be one of the highest dietary contributors of silica in a Western diet, especially in men. ${ }^{205,206}$

Exposure to crystalline silica associated with the inhalation of crystalline particulate is linked to various lung diseases including emphysema, COPD, lung cancer and fibrotic pneumoconiosis, more commonly known as silicosis. ${ }^{97}$ Silicosis is the hardening and scarring of lung tissue that results in the loss of lung function. ${ }^{207}$ It is a terminal, irreversible, fibrotic pulmonary disease, with no treatment or cure, with effects that will continue to develop and progress well after exposure has ceased.

For exposure to occur, crystalline silica has to be small enough to enable it to become airborne (mostly as a dust fraction) and inhaled into the alveoli region of the lungs. Many sizes of crystalline silica have the potential to become airborne if the conditions are right. Respirable crystalline silica (RCS) however, is the portion of airborne crystalline silica small enough to enter the gas-exchange regions of the lungs (alveoli) when inhaled. Therefore, the RCS particles must have an aerodynamic diameter less than approximately $10 \mu \mathrm{m} .{ }^{208}$ For instance, upon inhalation of airborne dust containing particles of crystalline silica there are three particle size fractions: inhalable, thoracic and respirable. The inhalable fraction is related to the largest particle sizes that are breathed into the nose and mouth only. The thoracic fraction relate to smaller particles that are able to penetrate the head airways and enter the airways of the lungs (bronchial tubes). The respirable fraction relates to smallest particles $(<10 \mu \mathrm{m})$ and it is this fraction, termed RCS, that is capable of penetrating beyond the terminal bronchioles and into the gas exchange region of the lungs (alveoli). 


\subsubsection{Silicosis}

Silicosis is believed to only be an occupational disease, as environmental levels of silica dust are not considered high enough to cause silicosis in the general background population. However, this is obviously dependent on environment and circumstance, as silicosis has been found in both men and woman of Himalayan villages due to frequent exposure to crystalline silica through dust storms. ${ }^{209}$

Silicosis is categorised into three types, with chronic silicosis being the most common. $^{207}$

1. Acute - Silicosis develops very quickly from a few months up to as long as 2 years after exposure. It results from exposure to very high levels of RCS over a short period of time.

2. Accelerated - Silicosis usually develops 5-10 years after exposure to high levels of RCS. The onset of symptoms is longer than with acute silicosis however, progression of the disease is fast.

3. Chronic - Silicosis usually develops from between 10 years up to 40 years after exposure. Exposure is in the form of long term low level exposure to RSC. ${ }^{207}$

Symptoms of silicosis are similar to many other lung disorders, which is one reason for under diagnosis of the disease in the past. Symptoms begin with shortness of breath, development of a cough, continuing to a fever, cyanosis (bluish discolouration of the skin around either the lips or tips of the fingers), loss of appetite, fatigue, pulmonary edema, pneumonia, chest pain and finally respiratory failure. ${ }^{201,210,211}$ In addition, sufferers of silicosis have an increased risk and susceptibility towards other health conditions such as mycobacterial infections, tuberculosis, autoimmune disease and lung cancer. ${ }^{201,212}$

After inhalation, RCS deposits in the lungs causing epithelial injury and macrophage activation, which in turn leads to inflammation. RCS persists and accumulates in the lungs which causes three reactions: ${ }^{97}$

1. The release of cytokines and growth factors from macrophages and epithelial cells 
2. The release of reactive oxygen and nitrogen intermediates

3. Stimulation of oxidative stress in the lungs

It is these three reactions or pathways, which contribute to silicosis and other lung diseases.

\subsubsection{Industrial \& Occupational Exposure to Silica}

Throughout history, occupational exposure in the form of inhalation of dusts has been linked to lung related diseases. Afflictions termed "miners' phthisis," "dust consumption," "mason's disease," "grinders' asthma," "potters rot" and "stonecutters disease" are all now collectively called silicosis. ${ }^{212}$ The number of industries and occupations where the potential for inhalation of RCS occurs is vast, as displayed in Table 9, however many of these industries and occupations are dependent on certain activities (outlined in Table 10) within the workplace to create the correct environment for RCS exposure.

Table 9 Occupations and industries where exposure to RCS occurs

\begin{tabular}{|c|c|c|}
\hline \multicolumn{2}{|c|}{ Industries \& Occupations ${ }^{200,201,208}$} \\
\hline Construction & Quarrying & Milling \\
\hline Mining & Tunnelling & Grindstone Production \\
\hline Tombstone production & Stone Mason & Foundries \\
\hline Boiler scaling & Agriculture & Fotteries \\
\hline Arts \& Sculpture & Jewellery Making & Recycling \\
\hline Road \& Highway & Ceramics & Shipbuilding \& Repair \\
\hline Auto-mobile repair & Hydraulic Fracturing & Fitting \\
\hline
\end{tabular}


Interestingly, high instances of RCS exposure and silicosis have been reported in dry cutting workers of artificial stone in ||$_{\text {srael }}{ }^{213}$ and the manufacturers of kitchen/bathroom worktops in Spain ${ }^{214,215}$, North America ${ }^{216}$ and an unspecified location, ${ }^{217}$ both of which had not previously been recognised as industries of RCS exposure.

Table 10 Workplace activities that create RCS when working with crystalline silica containing rock, soil, sand, gravel, resources and products

\begin{tabular}{|c|c|c|}
\hline \multicolumn{3}{|c|}{ Activities creating aerosolised dust containing RCS particulate ${ }^{200,201,208}$} \\
\hline Drilling & Breaking & Crushing \\
\hline Cutting & Sawing/Slicing & Abrasive blasting \\
\hline Sand blasting & Grinding & Sanding \& scaling \\
\hline Excavation \& Digging & Hammering & Casting \& Moulding \\
\hline Furnace installation & Dry sweeping & Pressurised air blowing \\
\hline Polishing \& Buffering & Mixing & Ploughing \& Harvesting \\
\hline Demolition & Blasting & Etching \& Engraving \\
\hline
\end{tabular}

\subsubsection{Biological Monitoring of Silica}

Unfortunately, biological monitoring is not available to assess and monitor exposure to RCS. This is mainly due to urinary levels of silica being so high, due to the sheer abundance of silica in the environment, diet and from general human lifestyle. Silica (measured as silicon) measurements at $\mathrm{mg} / \mathrm{L}$ concentrations have been reported in urine, $^{19,218,219}$ blood $^{219}$ and serum ${ }^{19}$ by either graphite furnace atomic absorption spectroscopy (GFAAS) or inductively coupled plasma atomic emission spectrometry (ICP-EAS) of control populations not occupationally exposed to silica. The fact that there is large inter-individual variation of background urinary silica levels within populations ${ }^{19,218}$ and that the excretion of an ingested silica dose is under 24 hours ${ }^{19}$ 
all add to the difficulty of occupational silica measurement. There is also the additional problem of how to distinguish between occupational RCS and the high levels of amorphous silica. Total silica in urine measured as silicon by inductively coupled plasma mass spectrometry (ICP-MS) at HSL has experienced an inability to distinguish differences between occupational samples and background population samples.

Analysis of silicon by ICP-MS also has its own challenges. Firstly, silicon is not analytically sensitive (i.e. has a high limit of detection) due to elevated background levels of silicon on an ICP-MS, meaning the change in response from background to stimulus is low. The natural abundance of silicon in the materials of the ICP-MS hardware, the quartz sample introduction set-up and analytical reagents all contribute to high background levels of silicon. Secondly, silicon has several polyatomic interferences $\left({ }^{1} \mathrm{H}^{27} \mathrm{Al},{ }^{14} \mathrm{~N}_{2},{ }^{12} \mathrm{C}^{16} \mathrm{O}\right)$ which collision/reaction cell technology cannot sufficiently reduce to achieve the low limits of detection usually associated with and expected of ICP-MS. Furthermore, RCS is soluble only in hydrofluoric acid or strong alkaline conditions such as potassium hydroxide and sodium hydroxide. Strong concentrations of sodium or potassium hydroxide are not conducive to ICP-MS analysis and hydrofluoric acid requires a specialist sample introduction system, together with platinum-tipped cones for ICP-MS use. In addition, insoluble particles in a liquid sample are not compatible with ICP-MS analysis. It is also important to note that it is not known whether urinary measurements would be representative of inhaled RCS that deposits and accumulates in the lungs after exposure.

The only way of assessing workplace exposure is environmental air monitoring of which there is an HSE workplace exposure limit (WEL) of $0.1 \mathrm{mg} / \mathrm{m}^{3}$ based on an 8 hour time weighted average. ${ }^{24}$ However, RCS levels in coal mines and mills, granite quarrying and processing other stone crushing industries, foundries, ceramic industries, construction and sandblasting operations frequently exceed the WEL. ${ }^{200}$ Therefore, limiting occupational exposure is fully reliant on engineering controls such as ventilation, extraction, suppression of dust and dust collection along with respiratory protective equipment (RPE) such as dust masks. However, with no biological monitoring, it is not known how effective any of these controls are both in 
terms of worker behaviour (for example correctly wearing RPE) or how successful they are at stopping or reducing exposure to the respirable fraction of RCS whose particle sizes are mostly not visible to the human eye. For example, it is unknown whether the smallest particles of RCS are able to penetrate through the tiny holes within the fabric of dust masks.

A global estimate suggests that 28000 people die annually from occupational exposure to $\mathrm{RCS}^{220}$ of which Britain contributes approximately 800 deaths, ${ }^{221}$ averaging 15 people a week. Exposure to RCS is a global problem and it is clear from the number of estimated annual deaths that the past, present and future process of assessing and controlling workplace exposure to reduce the risk of future ill health has been and will continue to be a difficult task for environmental monitoring alone. The development of a biological monitoring method to assess exposure to RCS would be a significant improvement in the battle to reduce the risk and occurrence of silicosis and other health effects associated with RCS exposure. Chapter 6 of this thesis investigates the initial stages of developing a method to measure RCS in EBC samples by ICP-MS, with the aim of establishing a biomonitoring method for RCS. 


\subsection{Speciation}

\subsubsection{Definition}

The International Union for Pure and Applied Chemistry (IUPAC) defined chemical species as chemical compounds that differ in isotopic composition, conformation, oxidation state or in the nature of their complexed or covalently bonded substituents. $^{222}$ This itemised distinction of a chemical species has led to three definitions to describe speciation:

1. Chemical Species - Chemical elements: specific form of an element defined as to isotopic composition, electronic or oxidative state, complex or molecular structure

2. Speciation Analysis - Analytical chemistry: analytical activities of identifying and/or measuring the quantities of one or more individual chemical species in a sample.

3. Speciation of an element - Distribution of an element amongst defined chemical species. ${ }^{222}$

Elemental speciation is the separation of an element into its different oxidation states or chemical forms and subsequent quantification. Elemental speciation is considered crucial when the toxicity of an element is dependent on its oxidation state or chemical form.

The speciation of elements such as chromium, arsenic and silicon is vital because the toxicity and biological availability of these and other elements depend on their different oxidative states or chemical compounds in addition to the quantity. For example, there have been over fifty identified arsenic species in the literature all with varying toxicities. ${ }^{95}$ For compounds associated with human occupational exposure, arsenic speciation not only separates oxidative states $\mathrm{As}^{3+}$ and $\mathrm{As}^{5+}$ but can also separate organic arsenic compounds such MMA and DMA. Chromium is an excellent example of the differing toxicities of two oxidation states of the same element and the importance of speciation to distinguish between an essential element and a 
carcinogen. The separation of crystalline silica from amorphous silica is an example of chemical composition speciation, in addition to further separation of crystalline silica by size.

It should be noted, the value of speciation and the separation and quantification of each individual species depends entirely on maintaining the integrity of the species throughout any extraction, dilution and analytical measurement.

\subsubsection{Speciation using Hyphenated Separation Techniques}

Elemental speciation is most commonly achieved by the hyphenation of a chromatographic separation technique to an elemental detection system. Such separation techniques include liquid chromatography (LC), gas chromatography (GC) and capillary electrophoresis (CE). ${ }^{223}$ Detection techniques are generally spectroscopy based and include inductively coupled plasma mass spectrometry (ICP-MS), ICP atomic emission spectrometry (ICP-AES) and atomic absorption spectroscopy (AAS). The most utilised elemental speciation technique is the hyphenation of LC to ICP-MS. The first study to couple LC and ICP-MS was published in $1986 .{ }^{224}$ It is considered the best combination ${ }^{225}$ because of its simplicity, robustness and reliability. ${ }^{223}$

In this work a range of analytical techniques were utilised. LC-ICP-MS was used for arsenic and chromium, time of flight tandem mass spectrometry (TOF-MS/MS) for arsenic and single particle ICP-MS for silica analysis. The next section will describe these techniques and the reason for their selection in more detail.

\subsubsection{Liquid chromatography (LC)}

The hyphenation of LC to ICP-MS is very straightforward; the column outlet is simply connected to the nebuliser of the ICP by narrow bore tubing, whose length should be kept to a minimum. Figure 7 shows a schematic of the components of LC-ICP-MS. Due to the small particle size of the stationary phase, the mobile phase must be pumped through the column, with the sample being introduced to the eluent via an injection port prior to being carried to the column where the separation of species takes place. 
Separation in LC depends on the interactions of the analyte with a stationary phase (contained in the chromatographic column) and the liquid mobile phase. The mobile phase can be either an isocratic or gradient elution. In an isocratic elution the concentration and/or constituents of the mobile phase are constant during the chromatographic run, as opposed to a change in mobile phase concentration and/or constituents associated with a gradient elution. The polarity, solubility, ionic charge and molecular mass of the elemental species of interest will determine the type of chromatographic column (stationary phase) and subsequently the type of mobile phase to be used. ${ }^{223,226}$

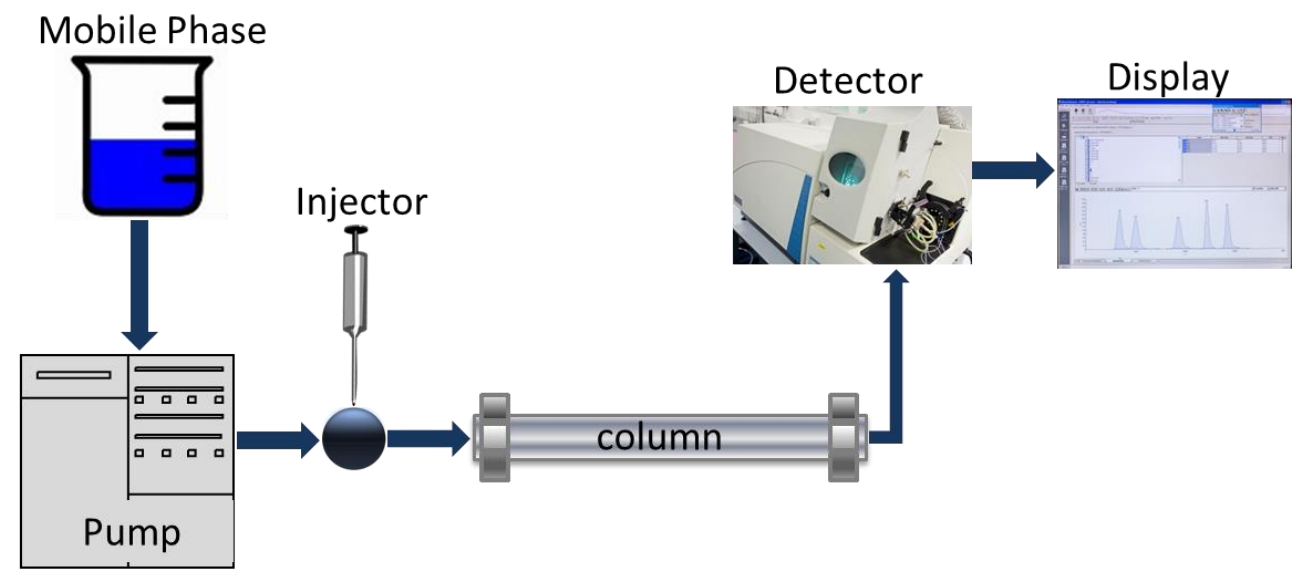

\section{Figure 7 Block diagram showing components of a traditional LC system coupled to a detector.}

\subsubsection{Columns and Stationary Phases}

The chromatographic columns that are commonly used for elemental speciation have included ion exchange, reverse phase and size exclusion based stationary phases.

Ion exchange columns normally contain stationary phases with charged organic functional groups, which are either anionic or cationic. Anionic and cationic columns can be categorised as either strong or weak. The functional group in a strong ion exchange column would mostly be quaternary amines and sulfonic acid for anion and cation exchange respectively. An example of weak ion exchange functional groups would be primary amines and organic acids, for anions and cations respectively. ${ }^{225,227}$ 
Therefore, ion exchange is suitable for the separation of ionic compounds based on charge, where species are retained on the stationary phase of the opposite charge. For example, the stationary phase of a cation exchange column has negatively charged sites which positively charged species interact with. The opposite is true for anion exchange, where negatively charged species interact with positively charged sites in the stationary phase.

Commonly used reverse phase columns are known as C18 or C8 columns, because they are packed with silica bonded to 18-carbon or 8-carbon aliphatic chains. This non-polar stationary phase works with a polar mobile phase to partition the elemental species of interest. Strongly retained species have a greater affinity for the stationary phase than the mobile phase. Different mixtures of solvents or a gradient elution with an increasing organic solvent content are common mobile phases for reverse phase chromatography to elute strongly retained species. Mobile phase $\mathrm{pH}$ can also influence separation. Reverse phase is the most commonly used column for traditional LC 225 mostly because it has a stable stationary phase, showing good resolution and high reproducibility. Unfortunately, this technique is limited because most organic solvents are not compatible with ICP-MS, reducing the scope and number of applications suitable for LC-ICP-MS. ${ }^{223,225}$

Size exclusion chromatography columns work on the premise of separation by hydrodynamic radius size. The stationary phase consists of a gel with pores of a specific dimension; species that are too large for these pores are not retained and elute from the column first. Smaller species that are able to interact with the pores of the stationary phase are retained. The mobile phases used in size exclusion chromatography do not play a critical role in the interactions of the stationary phase and the species of interest like the techniques mentioned above, as separation is effected by physical interactions between the species and the stationary phase. 


\subsubsection{Elemental Detection}

\subsubsection{Inductively Coupled Plasma Mass Spectrometry (ICP-MS)}

Inductively coupled plasma - mass spectrometry (ICP-MS) is the analytical technique used for the work reported in this study for the measurement of arsenic, chromium and silicon. ICP-MS is ideal due to its ability to: analyse liquid samples, low detection limits, capability of dealing with argon and polyatomic interferences and its selective range of elemental and isotopic choice.

ICP-MS can measure trace metals, metalloids and inorganic elements in liquid samples. The technique is relatively new compared to other atomic spectrometry instrumentation but has become the method of choice for elemental analysis as a result of it being a fast and accurate technique that can analyse, detect and measure either single or multi-elements in a single sample either semi-quantitatively or fullyquantitatively at low detection limits (ppt range). It does this by using an argon plasma to ionise atoms of the element to be detected. A quadrupole mass spectrometer then detects the ions by their mass to charge ratio $(\mathrm{m} / \mathrm{z})$.

Since the first publication introducing ICP-MS in $1980^{228}$ and the first available commercial ICP-MS in $1983^{229}$ this analytical technique, used to measure trace multielements, has greatly developed by: increasing sensitivity, further reducing detection limits and eliminating argon polyatomic interferences. As such, ICP-MS is widely and routinely used in a variety of different scientific fields such as clinical, industrial, environmental, geological and pharmaceutical. The dexterity of ICP-MS allows it to analyse practically all the elements in the periodic table with the exception of those located at the top right hand side of the periodic table (helium, carbon, nitrogen, oxygen, fluorine and neon) in addition to hydrogen and argon.

As demonstrated in Figure 8, an ICP-MS is comprised of four components: a sample introduction system, plasma generation, sampling interface and ion optics and the mass analyser/detector. 
An argon plasma is equivalent to a very hot flame operating at temperatures up to $7000^{\circ} \mathrm{C}$ and is generated by applying a radio frequency (RF) to a stream of argon gas. The stream of argon passes through a quartz torch that at one end is placed within a copper load coil. When the RF power is applied this creates a current that oscillates creating a magnetic field. An ignition spark is then applied to the argon gas, which strips electrons from argon atoms, which become trapped in the magnetic field. As argon gas continues to flow through the torch these electrons collide with more and more argon atoms. A schematic diagram of a plasma is shown in Figure 9. This continuing process of collisions enables a self-sustaining plasma to be created. This is called inductive coupling hence the name inductively coupled plasma. ${ }^{230}$

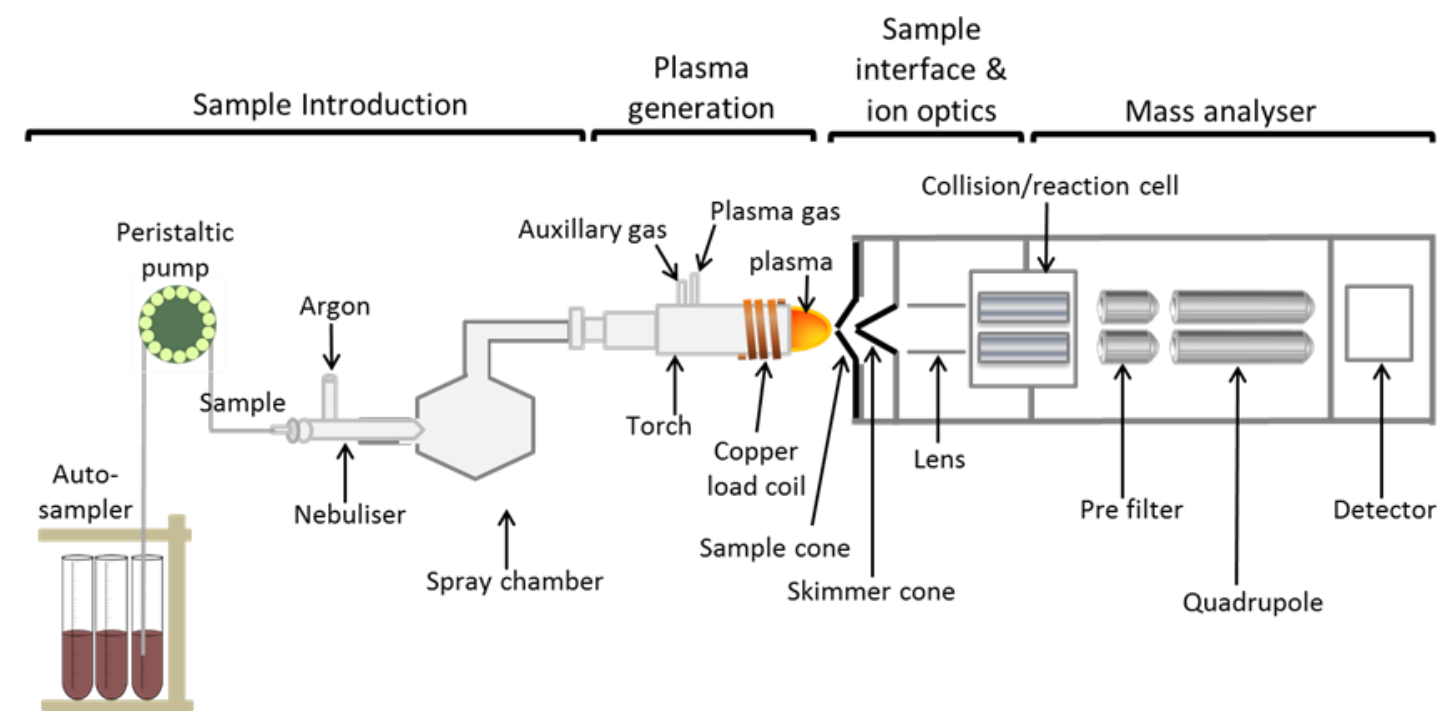

Figure 8 A schematic diagram of a quadrupole ICP-MS with collision/reaction cell 


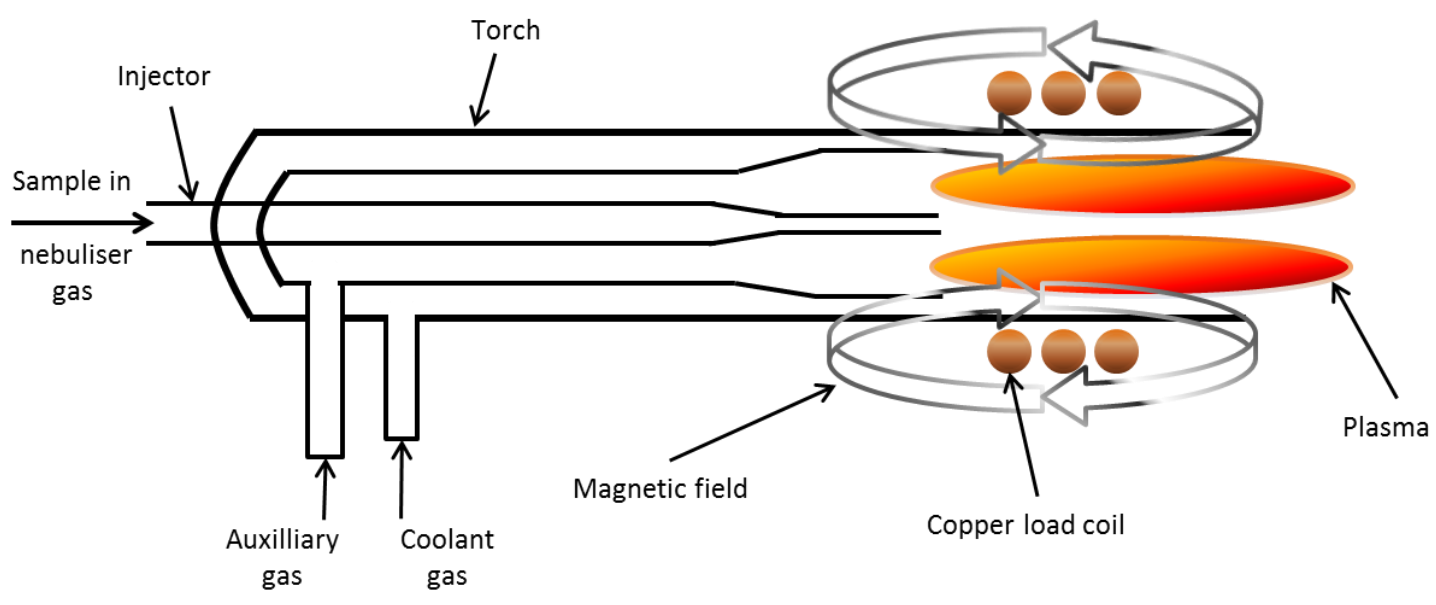

Figure 9 A schematic diagram of a plasma (adapted with permission from Nelms, 2005) $)^{230}$

Sample uptake is controlled by the peristaltic pump via a sample probe to the nebuliser and spray chamber. Within the nebuliser, the sample is met with a stream of argon gas, which causes the sample to aerosolise. Only $1-4 \%$ of the sample creates this aerosol ${ }^{231}$ (the rest going to waste). The argon then carries the aerosolised sample into the torch and the argon plasma where it is vaporised, atomised and ionised, to give positively charged ions.

The mass analyser section of the ICP-MS is kept under vacuum and to separate the high temperatures and pressure difference of the plasma from the vacuum of the MS an interface consisting of a sample and skimmer cone (of either nickel or platinum) plus a series of ion lenses is required. This interface allows only the positively charged ions to move into the MS (any negatively charged ions including electrons are discarded). ${ }^{232}$ The most common MS in an ICP is known as a quadrupole MS as it contains four parallel rods (as demonstrated in Figure 10) that hold opposing positive and negative charges for the ions to pass through. 


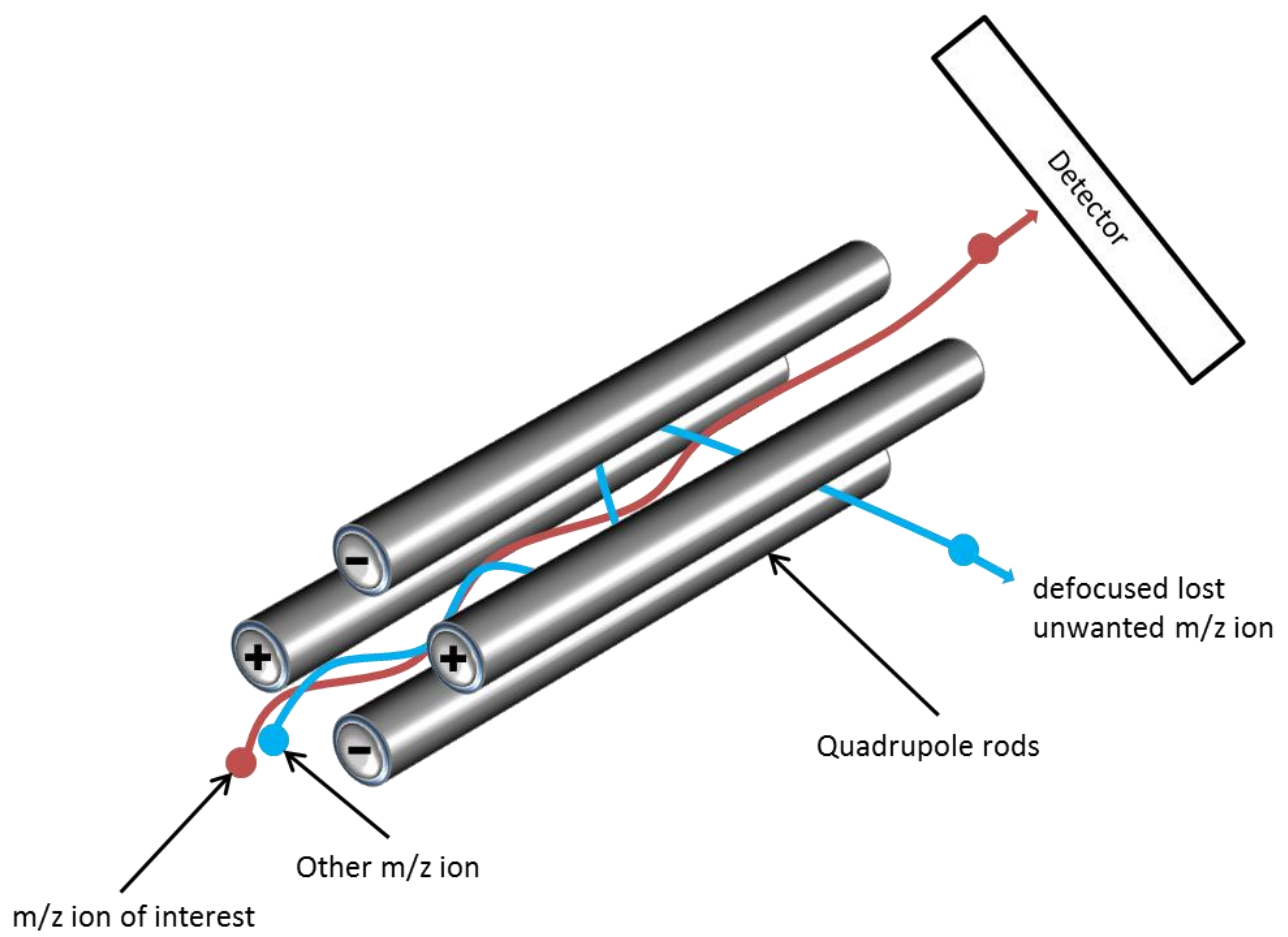

Figure 10 Quadrupole rods within the mass analyser section of an ICP-MS

The RF and direct current (DC) voltage charge on the rods is specific to the element of interest being detected, meaning only elements of a single $\mathrm{m} / \mathrm{z}$ find a stable trajectory to the detector and all other ions of a different $\mathrm{m} / \mathrm{z}$ are defocused within the rods and lost. ${ }^{232}$ Multi element analysis is achieved by changing the charge on the rods to favour each selected $\mathrm{m} / \mathrm{z}$ to allow sequential measurement of the different masses. ${ }^{232}$

One of the limitations of ICP-MS is spectral interferences. Whereas isobaric interferences can occur from isotopic overlap between the elements, polyatomic interferences occur when ions either from the plasma and/or other atoms in the sample matrix interfere with the ions of the element of interest, meaning the MS cannot differentiate between them (as shown in Table 11). To eliminate isobaric interferences, it may be possible to select an alternative elemental isotope that does not have the same influence of interference, for example ${ }^{111} \mathrm{Cd}$ instead of ${ }^{114} \mathrm{Cd}$ and ${ }^{118} \mathrm{Sn}$ instead of ${ }^{114} \mathrm{Sn}$. On the other hand, if the removal of the interfering ions from 
the sample matrix is not possible, polyatomic interferences can only be reduced by collision or reaction cell technology. The use of collision or reaction cells to eliminate interferences is commonplace in today's ICP-MS instrumentation and applications. The collision/reaction cell is positioned before the quadrupole of the MS. An inert gas is introduced (for example helium) which will either react with the interfering ion (and not the ions of interest) or collide with the polyatomic ions subsequently breaking bonds to produce single ion masses the quadrupole rods can discard, reducing the interference.

Table 11 Examples of isobaric and polyatomic interferences of ions seen in ICP-MS applications.

\begin{tabular}{|c|c|c|}
\hline $\begin{array}{c}\text { Element \& Mass of } \\
\text { Interest }\end{array}$ & $\begin{array}{c}\text { Polyatomic } \\
\text { Interferences }\end{array}$ \\
\hline${ }^{28} \mathrm{Si}$ & - & ${ }^{1} \mathrm{H}^{27} \mathrm{Al},{ }^{14} \mathrm{~N}_{2},{ }^{12} \mathrm{C}^{16} \mathrm{O}$ \\
\hline${ }^{52} \mathrm{Cr}$ & - & ${ }^{40} \mathrm{Ar}^{12} \mathrm{C},{ }^{36} \mathrm{Ar}^{16} \mathrm{O}$ \\
\hline${ }^{75} \mathrm{As}$ & - & ${ }^{40} \mathrm{Ar}^{35} \mathrm{Cl}$ \\
\hline${ }^{78} \mathrm{Se}$ & ${ }^{78} \mathrm{Kr}$ & ${ }^{14} \mathrm{~N}^{64} \mathrm{Zn}$ \\
\hline${ }^{80} \mathrm{Se}$ & ${ }^{80} \mathrm{Kr}$ & ${ }^{40} \mathrm{Ar}_{2},{ }^{40} \mathrm{Ar}^{40} \mathrm{Ca}$ \\
\hline
\end{tabular}

Table 11 shows some of the spectral interferences of ICP-MS applications and the polyatomic interferences of silicon, chromium and arsenic which are relevant to the ICP-MS applications discussed in this thesis.

\subsubsection{Single Particle ICP-MS}

Single particle ICP-MS (spICP-MS) analysis is a relatively new addition to the scope of ICP-MS. The first publication to test the theory of ICP-MS to analyse single particles (gold colloid) was by Degueldre et al in $2003,{ }^{233}$ however it was several years until interest in spICP-MS expanded. 
Natural and engineered particles are added to food, packaging, hygiene products and clothing in addition to widespread industrial uses. ${ }^{234}$ Therefore, the detection and characterisation of single particles or nanoparticles in both food, water and biological samples is considered essential in understanding the potential risks of exposure to both elemental engineered nanoparticles and single particle particulate ${ }^{235}$ such as respirable crystalline silica. The introduction of spICP-MS offers the potential to measure individual particles of a known elemental status.

As previously stated in section 1.6.3.1, the liquid sample to be analysed by the ICP-MS is aerosolised within the spray chamber before being vaporised, atomised and ionised in the plasma. During regular ICP-MS analysis the element of interest within a sample is homogenously distributed meaning that the ions produced in the plasma and travelling to the detector, do so at a constant rate producing a steady signal. However, with single particle analysis, the element of interest within the sample is not homogenously distributed and, as each single particle passes into the plasma, it is desolvated and ionised creating a cloud or plume of ions (rather than a continuous stream) which is measured as a single pulse.

To perform spICP-MS, the ICP-MS data are collected in time resolved analysis (TRA) mode, with a low flow rate and a much reduced data acquisition time (dwell time) of 3 ms. Regular ICP-MS analysis at the HSL would use a dwell of $100 \mathrm{~ms}$. Figure 11 demonstrates the difference in the detection of ions for a $1 \mu \mathrm{g} / \mathrm{L}$ ionic gold standard over an acquisition time of $60 \mathrm{~s}$ with a dwell time of $100 \mathrm{~ms}$ compared to that with a dwell time of $3 \mathrm{~ms}$.

Assuming the quantity of suspended particles in the sample is not too concentrated; this setup allows the ICP-MS to detect each individual pulse, which represents a single particle as shown in Figure 12. 

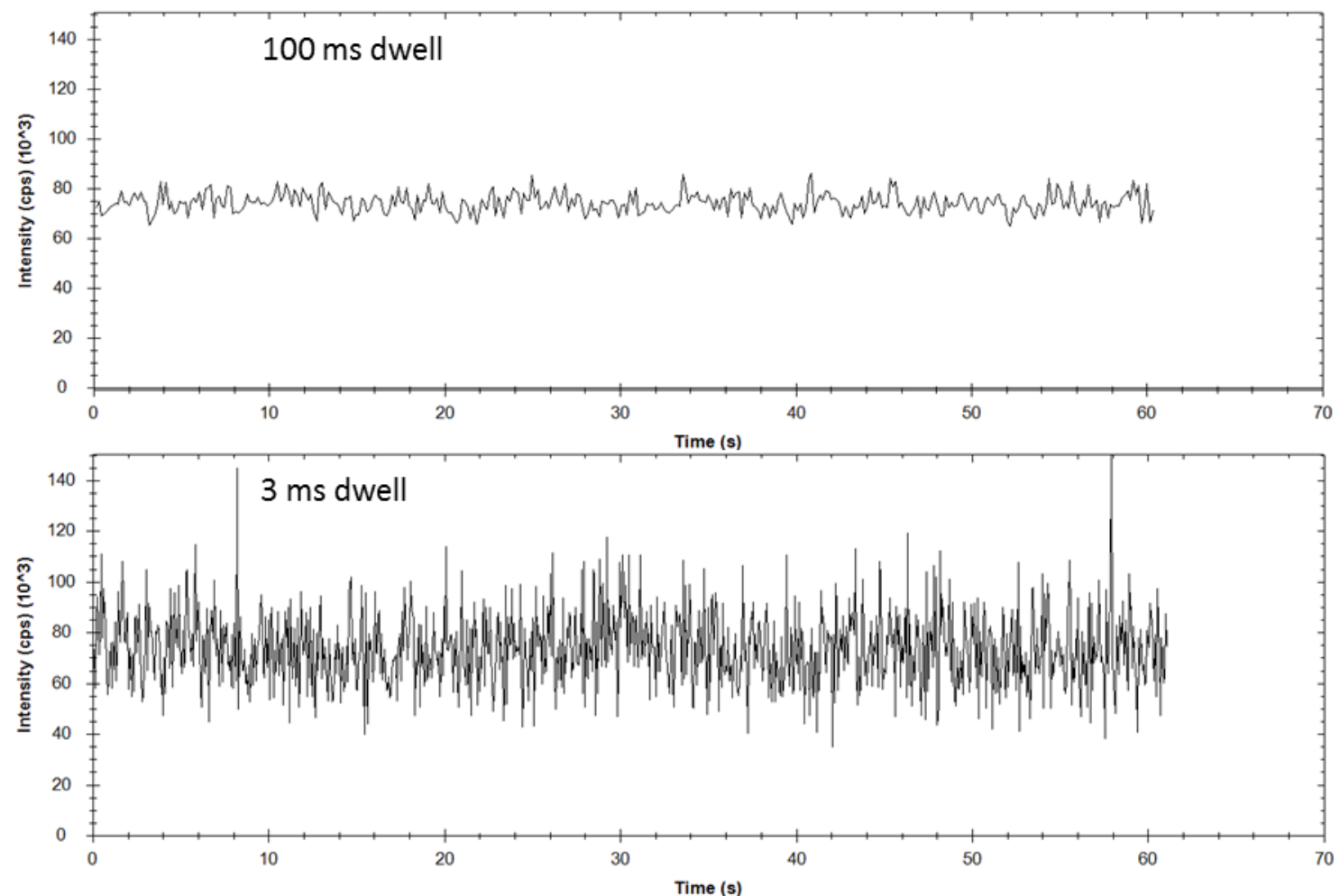

Figure 11 Time scan of a $1 \mu \mathrm{g} / \mathrm{L}$ ionic gold standard over a $60 \mathrm{~s}$ acquisition time, demonstrating the difference in the signal obtained by the detection of gold ions when the dwell time is $100 \mathrm{~ms}$ compared to $3 \mathrm{~ms}$ using spICP-MS.

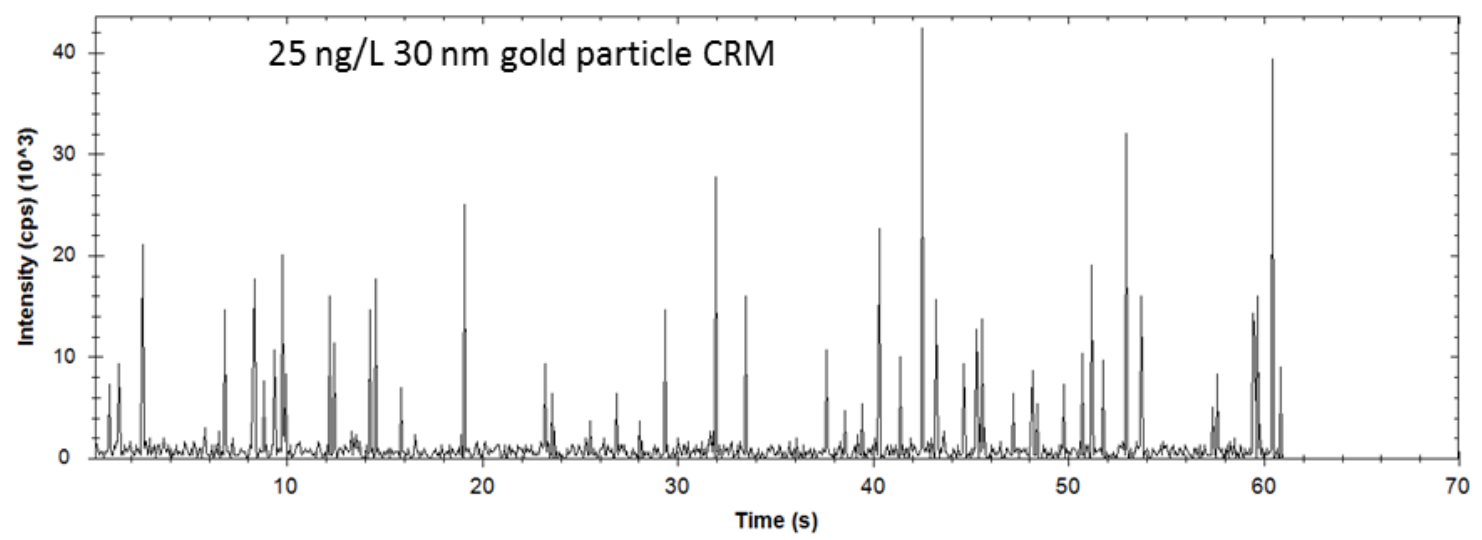

Figure 12 Time scan of a $25 \mathrm{ng} / \mathrm{L} 30 \mathrm{~nm}$ gold particle certified reference material using SpICP-MS. 
Therefore, the assumption is that the frequency of pulses is directly linked to the number concentration of particles, whilst the intensity of the pulse is proportional to the mass of the element. From the intensity of the pulse, a particle mass and diameter can be calculated from an ionic calibration curve and a known particle size reference material assuming the sample flow rate, nebulisation efficiency, density of the particle and the sensitivity of the ICP-MS for the element of interest in counts per second (cps) per $\mu \mathrm{g} / \mathrm{L}$ are already known. The limit of detection for spICP-MS is related to the size of the particle, as the detection is based on the single particle generating a large enough plume of ions to create a pulse detectable from the continuous background baseline, hence why peak height relates to particle size.

spICP-MS is a relatively new concept and although the ICP-MS hardware could measure single particles the software was not capable of calculating particle number concentration or particle size from the data it generated. Initial work on spICP-MS demanded offline data processing of numerous mathematical equations; the details to these approaches can be found in the literature. ${ }^{233,235-239}$ However, in 2015 many of the ICP-MS manufacturers launched single particle software as an update to existing software. Chapter 6 of this thesis discusses spICP-MS and its potential in the development of a biological monitoring method for RCS in EBC samples. The fundamentals of spICP-MS will be explained further in the discussion.

\subsubsection{Tandem Mass Spectrometry - MS/MS}

Tandem mass spectrometry, known as MS/MS, involves a multiple step of mass selection, with some form of mass fragmentation. The initial stage (MS1) of MS/MS is where an ion of interest is selected and measured according to its $\mathrm{m} / \mathrm{z}$. The second stage allows the selected ion to be subsequently broken down to its fragment or product ions by collision induced dissociation followed by detection, separation and measurement by the second MS of these fragment or product ions according to their $\mathrm{m} / \mathrm{z}$ (MS2). This production of fragment ions helps to provide structural and molecular information to aid identification of a polyatomic compound. The spectrum provided by 
MS2 would only consist of product ions from the initial ion of choice, with the elimination of any background or matrix components.

Collision induced dissociation is a similar concept to collision cell technology found in ICP-MS where the ion of interest is subjected to a neutral gas (argon, helium or nitrogen) causing collisions which break down the chemical bonds of the ion revealing the polyatomic compound's fragment ions.

\subsubsection{Time of Flight (TOF) MS/MS}

Time of flight (TOF) MS detects ions according to their $\mathrm{m} / \mathrm{z}$ by means of their flight times. ${ }^{240}$ The mass analyser region unlike in ICP-MS (where quadrupole rod's voltages change) operates under the same conditions. All the ions are subjected to an electric field giving the same kinetic energy to achieve a mass-dependent velocity to the detector. ${ }^{240,241}$ Lighter $\mathrm{m} / \mathrm{z}$ ions will achieve a higher velocity than heavier ions, ${ }^{240}$ resulting in measurements of the intensity and flight time producing a mass spectrum representative of all the ions present in the sample. ${ }^{241}$

A common ionisation technique for TOF is electrospray ionisation (ESI). The sample containing the element of interest is injected with a mobile phase, consisting usually of an organic-aqueous solvent (such as formic acid with methanol and water or acetonitrile and water) ${ }^{242}$ through a needle to create an aerosol. The needle is attached to a high voltage power supply, where the element of interest is ionised by gaining a proton (known as protonation) from the solvent it was injected with as it leaves the tip of the needle. This technique produces ions of a positive charge and mass $[\mathrm{M}+1]^{+}$. It is also possible to achieve negative ionisation $[\mathrm{M}-1]^{-}$with the use of ammonia solution as the mobile phase enabling deprotonation of the sample during ionisation. 


\subsubsection{Chromatographic Column and LC System used in this Study.}

\subsubsection{Dionex ${ }^{\mathrm{TM}}$ IonPac ${ }^{\mathrm{TM}}$ AG7 Anion Exchange Column}

The Dionex IonPAC AG7 (Thermo Scientific, Hemel Hempstead, UK) is a $4 \mathrm{~mm} \times 50 \mathrm{~mm}$, $10 \mu \mathrm{m}$ i.d anion exchange column ideally suited to the separation of polyvalent anions; polyvalent anions and chelating agents; and complex sample matrices. It is also marketed as a column suitable for the determination of cyanide, sulphide, iodide, hexavalent chromium and arsenic species. The IonPAC AG7 is suitable for eluents of $\mathrm{pH}$ $0-14$ with a maximum flow rate of $3.0 \mathrm{~mL} / \mathrm{min}$. It is marketed as a speciality packed column as it contains both anionic and cationic ion-exchange capabilities. The patented column packing consists of three regions:

- Inert, nonporous, chemically and mechanically stable core

- A core surface completely covered by a sulfonated region

- An outer layer of permanently attached submicron anion exchange MircoBeads ${ }^{\mathrm{TM}}$ with an alkyl quaternary ammonium functional group.

\subsubsection{Micro-flow Liquid Chromatography ( $\mu \mathrm{LC})$ System}

The oneFAST system was designed as a high throughput sample introduction system for ICP-MS or ICP-AES by Elemental Scientific (Warrington, UK). However, its automated sample injection valve designed to speed up sample uptake and rinse times was reminiscent of the rheodyne valve found on traditional HPLC systems, giving it the potential to be adapted as an LC system for speciation. For busy commercial laboratories, due to its size and the considerable price difference, the oneFAST system has some advantage over traditional LC systems. The oneFAST system comprises of a vacuum pump and small switching valve unit (Figure 13) which can easily fit between the auto-sampler and ICP-MS spray chamber (Figure 13) to enable coupling to the anion exchange column. 

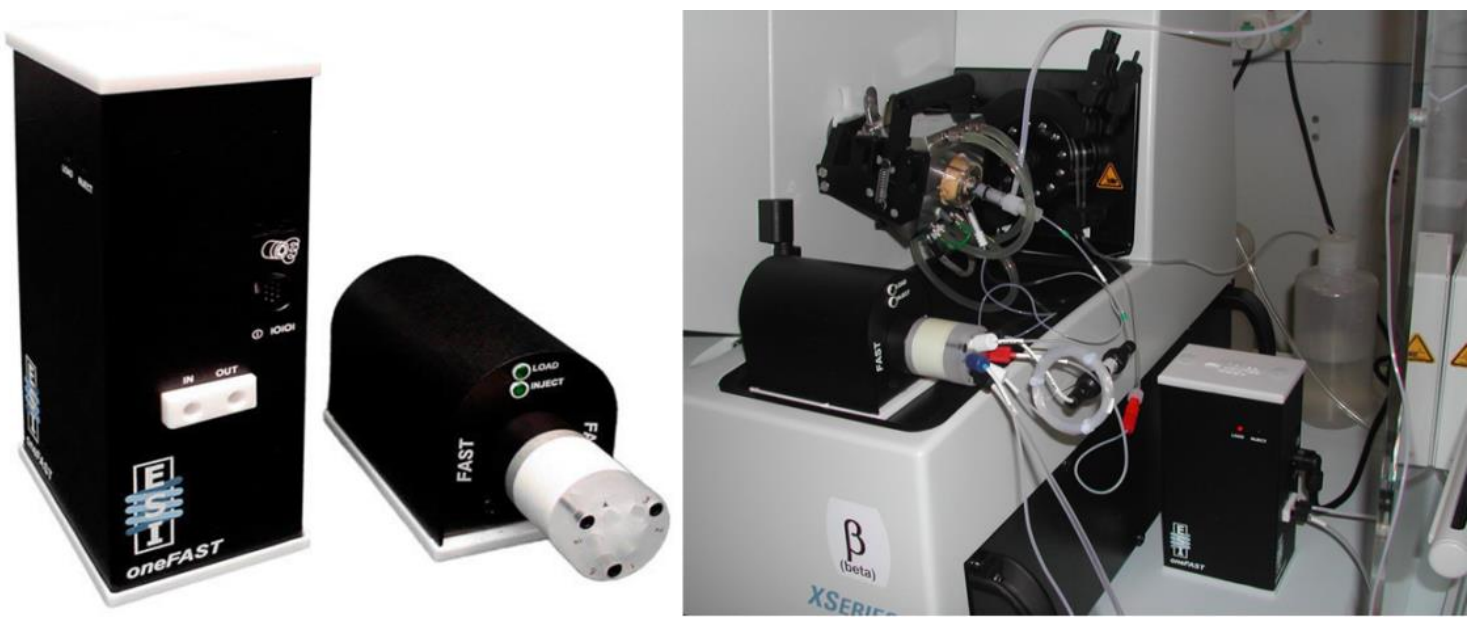

Figure 13 The oneFAST system comprising of a vacuum pump and switching valve unit (left) kindly supplied by Elemental Scientific. The oneFAST hyphenated to and, situated alongside the ICP-MS (right).

The switching valve of the oneFAST system consists of 6 ports allowing connections for: sample introduction; anion exchange column; mobile phase; waste and the sample loop (taking up two connections of the switching valve). A traditional LC system incorporates a LC pump to control the flow of mobile phase onto the column; the oneFAST system lacks this ability and so a constant flow rate of both mobile phase and injection of sample onto the column is controlled by the peristaltic-pump of the ICPMS.

The default position of the switching valves is SAMPLE LOAD; in this position, mobile phase is constantly taken up and injected onto the column and into the ICP-MS. The auto-sampler probe goes to the sample in the auto-sampler rack to be analysed and the sample is loaded into the sample loop by the vacuum pump, with any excess sample going to waste, as shown in Figure 14. When the sample loop is full, the switching valve shifts to SAMPLE INJECTION. As shown in Figure 14, in SAMPLE INJECTION the sample already loaded in the sample loop is injected onto the column and ICP-MS, followed by the mobile phase. When the length of time allocated to inject the sample onto the column has expired, the switching valve reverts to SAMPLE LOAD, any remaining sample still in the sample loop goes to waste and the mobile phase continues to be pumped onto the column as before. 

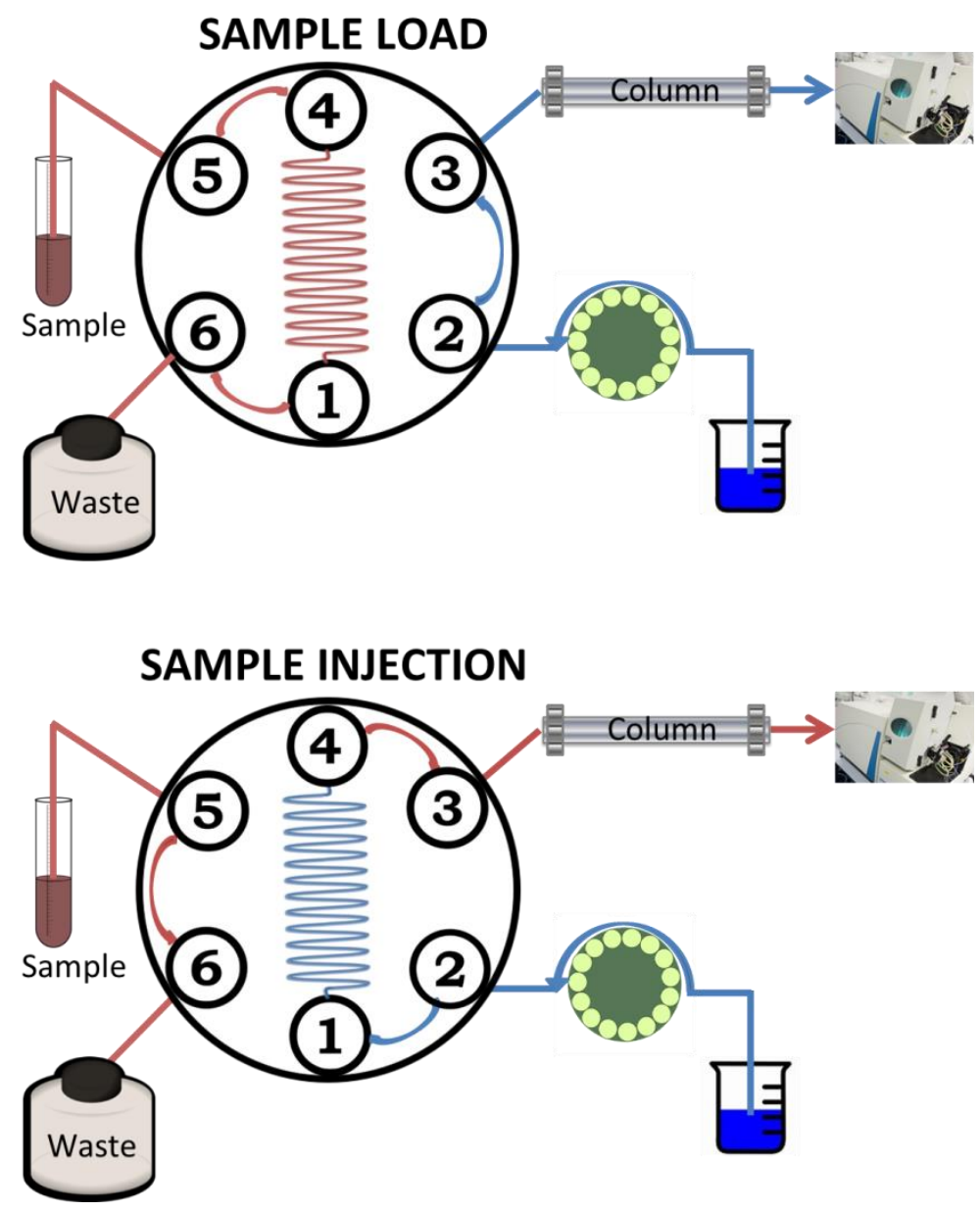

Figure 14 Switching valve positions of the oneFAST system, demonstrating uptake of mobile phase and sample

The advantages of the oneFAST system are that it works at low pressure, it uses smaller chromatographic columns and the analysis uses less mobile phase hence producing less waste. There are two limitations to the oneFAST system. Firstly, as the peristaltic-pump of the ICP-MS is controlling the flow rate onto the column, enough pressure cannot be generated on longer length chromatographic columns to pump the sample and mobile phase through the column; in addition the pressure cannot be monitored like a traditional LC system. The second limitation is the oneFAST system is only capable of an isocratic mobile phase or a step gradient mobile phase. A linear gradient mobile phase is not possible, however Elemental Scientific have since rectified this issue with the development of numerous improved speciation specific products. In the system used here, the use of a gradient mobile phase was achieved by injecting a different mobile phase onto the column in the same way the sample was injected. 


\subsection{Aims \& Objectives}

This work was funded by the Health \& Safety Executive and the Health \& Safety Executive's Health \& Safety Laboratory in an effort to further biological monitoring research of elements which are of carcinogenic, occupational concern: inorganic arsenic, hexavalent chromium and respirable crystalline silica. Current biological monitoring methods are either no longer deemed fit for purpose (arsenic), not ideal (chromium) or are not available (silica).

The aims of this PhD project were:

1. To develop new analytical speciation methods for arsenic, chromium and silica, to assess occupational exposure.

2. To evaluate exhaled breath condensate, for its potential as a new biological matrix for speciation analysis of chromium and, single particle analysis of respirable crystalline silica.

To achieve these aims, the following objectives were set:

- To develop an arsenic speciation method that can be employed for routine analysis and to establish an unexposed background reference range for each of the five species of arsenic to aid the interpretation of biological monitoring results.

- To develop a chromium speciation method to simultaneously detect and separate both hexavalent and trivalent chromium during a single sample injection of the same analysis.

- To investigate exhaled breath condensate as a potential biological matrix for the exposure assessment of hexavalent chromium and respirable crystalline silica.

- To develop a methodology using spICP-MS to detect and separate respirable crystalline silica in exhaled breath condensate samples, in an effort to quantify particle number and particle size. 


\subsection{Publications Outlined in the Chapters of this Thesis}

Chapter $2 \quad \mu$ LC-ICP-MS determination of unexposed UK urinary arsenic speciation reference values.

E. Leese, J. Morton, E. Tan, P.H.E. Gardiner and V.A. Carolan. J. Anal. Toxicol., 2014, 38(1): 24-30.

Chapter 3 The investigation of unexpected arsenic compounds observed in routine biological monitoring urinary speciation analysis.

E. Leese, M. Clench, Morton, J. P.H.E. Gardiner and V.A. Carolan. Toxics. 2017, 5(2): doi:10.3390/toxics5020012

Chapter 4 Development of a method for the simultaneous detection of $\mathrm{Cr}(\mathrm{III})$ and $\mathrm{Cr}(\mathrm{VI})$ in exhaled breath condensate samples using $\mu \mathrm{LC}-\mathrm{ICP}-\mathrm{MS}$.

E. Leese, J. Morton. P.H.E. Gardiner and V.A. Carolan. J. Anal. At. Spectrom., 2016, 31(4): 924-933.

Chapter 5 The simultaneous detection of trivalent and hexavalent chromium in exhaled breath condensate: A feasibility study comparing workers and controls.

E. Leese, J. Morton, P.H.E. Gardiner and V.A. Carolan. Int. J. Hyg. Environ. Health., 2017, 220: 415-423.

Chapter 6 Exhaled breath condensate: A novel matrix for biological monitoring to assess occupational exposure to respirable crystalline silica.

E. Leese, J.F. Staff, V.A. Carolan and J. Morton. Ann. Work. Exp. Health. (formerly Ann. Occup. Hyg.), 2017, 61(7): 902 - 906. DOI: 10.1093/annweh/wxx047 


\subsection{Disclaimer}

The published studies reported in the results chapters of this thesis have been published as full open access and, were performed and written by the author of this thesis, except in the circumstances outlined below.

Chapter 2 - E. Leese, J. Morton, E. Tan, P.H.E. Gardiner and V.A. Carolan. LLC-ICP-MS determination of unexposed UK urinary arsenic speciation reference values. J. Anal. Toxicol., 2014, 38(1): 24-30.

The main statistical analysis and the Bayesian Regression Model were performed by Emma Tan (Statistician, Data Analytics Team, Health \& Safety Executive's Health \& Safety Laboratory, UK). However, Mann-Whitney statistical tests using Graphpad Prism were performed by the author of this thesis.

Chapter 3 - E. Leese, M. Clench, Morton, J. P.H.E. Gardiner and V.A. Carolan. The investigation of unexpected arsenic compounds observed in routine biological monitoring urinary speciation analysis. Toxics., 2017, 5(2): doi:10.3390/toxics5020012

Electrospray ionisation (ESI-qQ-TOF-MS/MS) analysis was conducted at Sheffield Hallam University by Professor Malcolm Clench.

Chapter 6 - E. Leese, J.F. Staff, V.A. Carolan and J. Morton. Exhaled breath condensate: A novel matrix for biological monitoring to assess occupational exposure to respirable crystalline silica. Ann. Work. Exp. Health. (formerly Ann. Occup. Hyg.), 2017, 61(7): 902 - 906. doi: 10.1093/annweh/wxx047

The transmission electron microscopy (TEM) analysis was performed by James Staff (Higher Analytical Scientist, Biological Monitoring Team, Health \& Safety Executive's Health \& Safety Laboratory, UK). The corresponding author of the publication was Dr Jackie Morton. The author of this thesis wrote the spICP-MS methodology and results sections. 


\section{Chapter 2.}

$\mu \mathrm{LC}$-ICP-MS Determinations of Unexposed UK urinary Arsenic Speciation Reference Values

E. Leese, J. Morton, E. Tan, P.H.E. Gardiner and V.A. Carolan. J. Anal. Toxicol., 2014, 38(1): 24-30.

Published - Government Open Access Licence

https://academic.oup.com/jat/article/38/1/24/830716/LC-ICP-MS-Determinations-ofUnexposed-UK-Urinary 


\title{
$\mu L C-I C P-M S$ Determinations of Unexposed UK Urinary Arsenic Speciation Reference Values
}

\author{
Elizabeth Leese $^{1,2 *}$, Jackie Morton ${ }^{1}$, Emma Tan ${ }^{1}$, Philip H.E. Gardiner ${ }^{2}$ and Vikki A. Carolan ${ }^{2}$ \\ ${ }^{1}$ Health and Safety Laboratory, Harpur Hill, Buxton, Derbyshire SK17 9JN, UK and ${ }^{2}$ Biomedical Research Centre, Sheffield Hallam \\ University, Howard Street, Sheffield S1 1WB, UK
}

"Author to whom correspondence should be addressed. Email: liz.leese@ hsl.gsi.gov.uk

This study provides background levels for five arsenic species in urine, based on urinary data obtained from 95 nonoccupationally exposed volunteers based in the UK. Using a novel, sensitive, robust and reliable speciation methodology, five species of arsenic (arsenobetaine $[A B]$, arsenite $\left[\mathrm{As}^{3+}\right]$, arsenate $\left[\mathrm{As}^{5+}\right]$, monomethylarsonic acid $\left[\mathrm{MMA}^{5+}\right]$ and dimethylarsinic acid $\left[\mathrm{DMA}^{5+}\right]$ ) were determined in urine samples collected from 95 adults. The analytical instrumentation used to analyze the urine samples was a hyphenated micro liquid chromatography $(\mu \mathrm{LC})$ system coupled to an inductively coupled plasma mass spectrometry (ICP-MS). Separation was achieved using an anion exchange micro-sized column. The results presented give the 95th percentile of concentrations, both uncorrected for creatinine $(\mu \mathrm{g} / \mathrm{L})$ and creatinine corrected $(\mu \mathrm{mol} / \mathrm{mol})$ in urine for the 95 volunteers. Statistical analysis was performed on the dataset using a Bayesian model to determine and quantify effects of gender, smoking and diet. The statistical results show that the consumption of fish, shellfish and red wine has a significant elevating effect on AB, DMA and MMA urinary concentrations; however, no significant effect was observed for smoking. The regression model results indicate that creatinine correction was effective for arsenic species $\mathrm{As}^{3+}, \mathrm{MMA}$, DMA and $\mathrm{AB}$. The background levels established here can be used as reference values to help aid interpretation of arsenic speciation results and better assess exposure.

\section{Introduction}

The International Agency for Research on Cancer (IARC) has classified inorganic arsenic as a Group 1 agent, defined as carcinogenic to humans (1). Thus, it is imperative that exposure be adequately controlled. Biological monitoring assesses whether a person has been exposed to a substance or its metabolites, as it encompasses all routes of potential exposure. Inorganic arsenic exists as arsenite (trivalent, $\mathrm{As}^{3+}$ ) and arsenate (pentavalent, $\mathrm{As}^{5+}$ ). When humans are exposed to inorganic arsenic, the arsenic is reduced and methylated in the body to produce a range of organic arsenic species, including dimethylarsinic acid $\left(\mathrm{DMA}^{5+}\right)$ and monomethylarsonic acid $\left(\mathrm{MMA}^{5+}\right)$ (2). Pentavalent methylated species are considered relatively nontoxic, since it is thought that they cannot bind with molecules in the body (3). In the UK, the most common route of exposure to arsenic is from dietary sources. Dietary species of arsenic known as tetra alkylarsonium compounds include arsenobetaine (AB), arsenocholine and trimethylarsine oxide, and are thought to be nontoxic (4). The dietary sources of these species of arsenic include seafood, shellfish, poultry and rice (4-8), and following ingestion, they are eliminated in the urine unchanged (4).

Urine is the main excretory pathway for elimination of arsenic from the body, in the general proportion $10-30 \%$ of inorganic arsenic, $10-20 \%$ of $\mathrm{MMA}^{5+}$ and $60-80 \%$ of $\mathrm{DMA}^{5+}$ are eliminated primarily via this route after inorganic exposure (2). The ease and noninvasive sample collection makes it the biological sample of choice in most studies, although determination of arsenic levels in blood $(9,10)$ and serum $(11)$ has been reported.

Currently, there are no unexposed background reference values or exposed UK guidance values for any individual arsenic species in urine. The US biological exposure index (BEI) (12) and the German biological tolerance value (BAT) (13) are 35 and $50 \mu \mathrm{g} / \mathrm{L}$, respectively, which are based on the summation of inorganic arsenic and its metabolites, but not including $\mathrm{AB}$. This is a controversial, less well-understood area, because there seems to be increasing numbers of foodstuffs containing other arsenic compounds, e.g., DMA $(6,7)$, and therefore, exposure to arsenic compounds either from environmental or from occupational sources could be overestimated. The BEI and BAT approach of the sum of the species less $\mathrm{AB}$ is an attempt to correct for dietary exposure, but does not account for dietary DMA, MMA and arsenosugars. To better assess environmental and occupational exposure, arsenic speciation must be undertaken as a routine assay.

There are several population studies from around the world that have reported urinary arsenic concentrations in unexposed individuals. The two most comprehensive are the National Health and Nutrition Examination Survey (NHANES) (14) in the USA, where 2557 participants' urine samples were measured for seven species of arsenic, and the Human Biomonitoring of Environmental Chemicals in Canada (HBECC) (15), where $~ 6400$ participants' urine samples were measured for six species of arsenic. In addition, similar national surveys have been conducted in France $(n=2102$ participants) (16) and Korea ( $n=5087$ participants) (17). However, both these studies used a total inorganic arsenic and the metabolites minus $\mathrm{AB}$ technique to measure arsenic $(16,17)$. There is also urinary arsenic speciation data for unexposed individuals in smaller scale clinical studies from around the world such as Germany (18), UK (19, 20), Italy (21) and Spain (22).

It is generally accepted that urinary arsenic speciation provides the most coherent and detailed picture of exposure to arsenic species and compounds. However, there is still a lack of established reference values to use when trying to assess exposure to individual species. By improving and modifying a novel micro liquid chromatography ( $\mu \mathrm{LC}$ )-inductively coupled plasma mass spectrometry (ICP-MS) speciation method, previously developed at the Health and Safety Laboratory (20) to have greater sensitivity and lower limits of quantification (LOQs), this paper aims to provide reference background values of five arsenic species in the urine of unexposed individuals from a cohort of UK samples. Information on diet and lifestyle was collected by questionnaire, as exposure to arsenic can occur through diet and environmental sources. Such data will provide a valuable contribution to the knowledge in this area. 


\section{Experimental}

\section{Instrumentation}

Separation was achieved using a hyphenated $\mu \mathrm{LC}$ system with an ICP-MS (XSERIES 2, Thermo Fisher Scientific, Hemel Hempstead, UK). The separation of arsenic species $\mathrm{As}^{3+}, \mathrm{As}^{5+}, \mathrm{MMA}^{5+}$, $\mathrm{DMA}^{5+}$ and $\mathrm{AB}$ was achieved using a $5-\mathrm{cm}$ anion exchange guard column (Dionex AG7 $4 \mathrm{~mm} \times 50 \mathrm{~mm}$ i.d., Thermo Fisher Scientific). The micro-flow delivery of sample and mobile phase was accomplished using an ESI OneFAST system (Elemental Scientific, Warrington, UK), which consists of a six port switching value and a $1-\mathrm{mL}$ sample loop. The delivery of mobile phase and sample onto the column was controlled by the ICP-MS peristaltic pump at a constant rate of $0.2 \mathrm{~mL} / \mathrm{min}$. The ICP-MS was operated in a collision cell mode using $7 \%$ hydrogen in helium $(\sim 3.5 \mathrm{~mL} / \mathrm{min})$. The ICP-MS conditions were optimized using a $10-\mu \mathrm{g} / \mathrm{L}$ tuning solution containing arsenic, cobalt, indium and uranium [made in $1 \%(\mathrm{v} / \mathrm{v})$ nitric acid from $1000 \mathrm{mg} / \mathrm{L}$ stock standards (ICP-MS standards, BDH, Poole, UK)].

\section{Reagents}

Arsenic speciation compounds sodium arsenite $\left(\mathrm{NaAsO}_{2}\right)$, sodium arsenate $\left(\mathrm{Na}_{2} \mathrm{HAsO}_{4} \cdot 7 \mathrm{H}_{2} \mathrm{O}\right)$ and sodium cacodylate (DMA; $\mathrm{Na}\left(\mathrm{CH}_{3}\right)_{2} \mathrm{AsO}_{2} \cdot 3 \mathrm{H}_{2} \mathrm{O}$ ) were all purchased from Fisher Scientific (Loughborough, UK). Disodium methyl arsenate (99\% MMA; $\mathrm{CH}_{3} \mathrm{AsO}(\mathrm{OH})_{2}$ ) was purchased from ChemService (West Chester, PA, USA). $\mathrm{AB}\left(\mathrm{C}_{5} \mathrm{H}_{11} \mathrm{AsO}_{2}\right)$ was purchased from Sigma-Aldrich (Dorset, UK). Single standard stock solutions of $1000 \mathrm{mg} / \mathrm{L}$ were prepared in $1 \%(\mathrm{v} / \mathrm{v})$ nitric acid (Romil, Cambridge, UK) of $\mathrm{As}^{3+}, \mathrm{As}^{5+}$, MMA, DMA and $\mathrm{AB}$ in plastic volumetrics. All stock solutions were stored at $4^{\circ} \mathrm{C}$. The separation of five arsenic species used a mobile phase of ammonium carbonate purchased from VWR (Leicestershire, UK). Two mobile phases were prepared daily by dissolving ammonium carbonate in ultrapure deionized water $(18.2 \mathrm{M} \Omega \mathrm{hm} \mathrm{cm})$ from a Millipore system (Merck Millipore, Billerica, MA, USA) to make $2 \mathrm{mM}$ (mobile Phase A) and $70 \mathrm{mM}$ (mobile Phase B) solutions.

A 1-mg/L mixed species solution of all the five species was prepared in $1 \%(\mathrm{v} / \mathrm{v})$ nitric acid from the individual 1000-mg/L single standard stock solutions on a weekly basis. Using the $1-\mathrm{mg} / \mathrm{L}$ mixed species solution standards of $0.5,1,2,5,10$ and $20 \mu \mathrm{g} / \mathrm{L}$ were prepared daily in $2 \mathrm{mM}$ of ammonium carbonate (mobile Phase A).

\section{Study group and sample collection}

The volunteers in this study $(n=95)$ consisted of 53 males and 42 females who were not occupationally exposed to arsenic. All volunteers were over the age of 18 years, with the mean age of people in this study 41.1 years. Individuals volunteered in response to an email outlining the study sent to everyone at the Health and Safety Laboratory (HSL), UK, and the Biomedical Research Centre, Sheffield Hallam University, Sheffield, UK. Participating volunteers were provided with informed consent, as granted by the NRES Committee East Midlands-Leicester, REC number 12/EM/0314. Volunteer information was collected via a questionnaire, which was returned by post with the urine sample. The information collected included: gender, age, smoking habits and diet including vitamin and supplement use in the previous 7 days. All urine samples were collected in $25 \mathrm{~mL}$ urine collection bottles (Sterilin Ltd., Newport, UK) and sent to the HSL by first class post. All urine samples had creatinine content analyzed upon arrival and were then kept frozen at $-80^{\circ} \mathrm{C}$ until analysis.

\section{Sample preparation and analysis}

Urine samples were thawed and mixed on a roller mixer before analysis. Samples were diluted 1 in 15 with $2 \mathrm{mM}$ ammonium carbonate solution. All samples were analyzed in duplicate.

The certified reference materials (CRMs) used were Clinchek urine control for arsenic species Level 1 and 2 (Lot 923) (Recipe, Munich, Germany). In addition, a blank urine and spiked urine sample (blank urine with all the five species of arsenic added at a concentration of $10 \mu \mathrm{g} / \mathrm{L}$ ) was analyzed at the beginning and the end of each series of analyses and after every 10 samples. External quality assurance for four of the arsenic species (not $\mathrm{AB}$ ) was undertaken in November 2012 by participation in the G-EQAS (Friedrich-Alexander-University, Erlangen-Nuremberg, Germany) trace element quality schemes.

Urinary creatinine concentrations were determined on all urine samples by the Jaffe alkaline picrate method (23) on a Cobas Mira Plus (Horiba Diagnostics, Northampton, UK). Creatinine (a breakdown product of creatine phosphate in muscle, and is usually produced at a fairly constant rate by the body) allows a urine sample to be normalized for dilution or concentration. In determining the creatinine levels using mathematical modeling, it was possible to investigate the effectiveness of this correction for each arsenic species.

\section{Statistical analysis}

The relationship was modeled between urinary concentration and several factors (including gender, smoking and dietary habits) using a multiple regression model. The Bayesian regression model used in this study had the form

$$
\begin{aligned}
\operatorname{1n}\left(\operatorname{As}_{i}\right)= & \mu+\beta_{1} 1 \text { n }\left(\text { creatinine }_{i}\right)+\beta_{2} I\left(\text { smoking }_{i}\right)+\beta_{3} I\left(\text { gender }_{i}\right) \\
& +\beta_{4} I\left(\text { supplements }_{i}\right)+\beta_{5} I\left(\text { fish }_{i}\right)+\beta_{6} I\left(\text { shellfish }_{i}\right) \\
& +\beta_{7} I\left(\text { seaweed }_{i}\right)+\beta_{8} I\left(\text { rice }_{i}\right)+\beta_{9} I\left(\text { mushrooms }_{i}\right) \\
& +\beta_{10} I\left(\text { red wine }_{i}\right) \beta_{11} I\left(\text { realale }_{i}\right)+\beta_{12} I\left(\text { poultry }_{i}\right) \\
& +\beta_{13} I\left(\text { (ricemilk }_{i}\right) \beta_{14} I\left(\operatorname{bran}_{i}\right)+\varepsilon_{i}
\end{aligned}
$$

where $\mathrm{As}_{i}$ is the urinary concentration of arsenic on the $i$ th individual; $\mu$, the mean concentration (on the log scale) of a nonsmoking female who has not taken dietary substances represented in the model; creatinine ${ }_{i}$, the creatinine concentration with associated effect $\beta_{1} ; I\left(\right.$ smoker $\left._{i}\right)=0$, if sample was from a nonsmoker and 1 if smoker $\left(\beta_{2}\right.$ represents the smoking adjustment to the mean concentration); $I\left(\right.$ gender $\left._{i}\right)=0$, if the urine sample was from a female and 1 , if male $\left(\beta_{3}\right.$ represents the male adjustment). Indicator variables and adjustments for the intake of supplements, fish, shellfish, seaweed, rice, mushrooms, red wine, real ale, poultry, rice milk and bran are similarly represented. $\varepsilon_{i}$ are normally distributed residual errors with mean zero and standard deviation $\sigma$.

One of the difficulties in analyzing urinary data is that measurements are often below the LOQ (referred to as nondetects). 
However, it is known that these measurements lie between zero and the LOQ, i.e., they are left censored. The data were modeled using Markov Chain Monte Carlo (MCMC) methods in WinBUGs (24) within a Bayesian framework, treating the nondetects as left-censored.

\section{Results}

\section{Analytical method}

The method described in this study is a sensitive, robust and reproducible method for the routine determination of five species of arsenic in a urine sample. The chromatograms in Figure 1 show that this optimized speciation method achieves full separation in $<6 \mathrm{~min}$. The calibration range was $0-20 \mu \mathrm{g} / \mathrm{L}$ for all the five species of arsenic. The limit of detection (LOD) was calculated as three times the standard deviation of the blank; this was between 0.003 and $0.051 \mu \mathrm{g} / \mathrm{L}$ for all the five species of arsenic. The LOQ for undiluted urine was calculated as 15 times the mean background equivalent concentration (BEC) for all the five species of arsenic. The BEC, LOD and LOQ for each arsenic species are summarized in Table I. Of the 95 volunteer urine samples measured, no level was below the LOQ for DMA and only $1 \%$ of $\mathrm{AB}, 2 \%$ of MMA and $12 \%$ of $\mathrm{As}^{3+}$ were less than the LOQ. However, $63 \%$ of the $\mathrm{As}^{5+}$ values were less than the LOQ of $0.04 \mu \mathrm{g} / \mathrm{L}$

\section{Analytical validation}

The results for the in-house spiked urine quality control sample shown in Table II gave an average recovery of $93 \%$ for $\mathrm{As}^{5+}$, MMA, DMA and $\mathrm{AB}$ and $90 \%$ for $\mathrm{As}^{3+}$ in the daily spiked urine samples $(n=29)$. The external quality assurance and CRMs analyzed are presented in Table II. Clinchek Levels 1 and 2 are certified for arsenic species; analyses gave good results for arsenic species $\mathrm{As}^{5+}$, MMA, DMA and AB, which were all within target range, whereas $\mathrm{As}^{3+}$ did not show good agreement and gave a result below the target range. However, the method is continually validated through participation in the German External Quality Assessment Scheme (GEQAS); the results for the 50th GEQAS in November 2012 being shown in Table II. The results were within the target criteria for all the five species of arsenic. This may suggest possible instability of $\mathrm{As}^{3+}$ in the Clinchek CRMs.
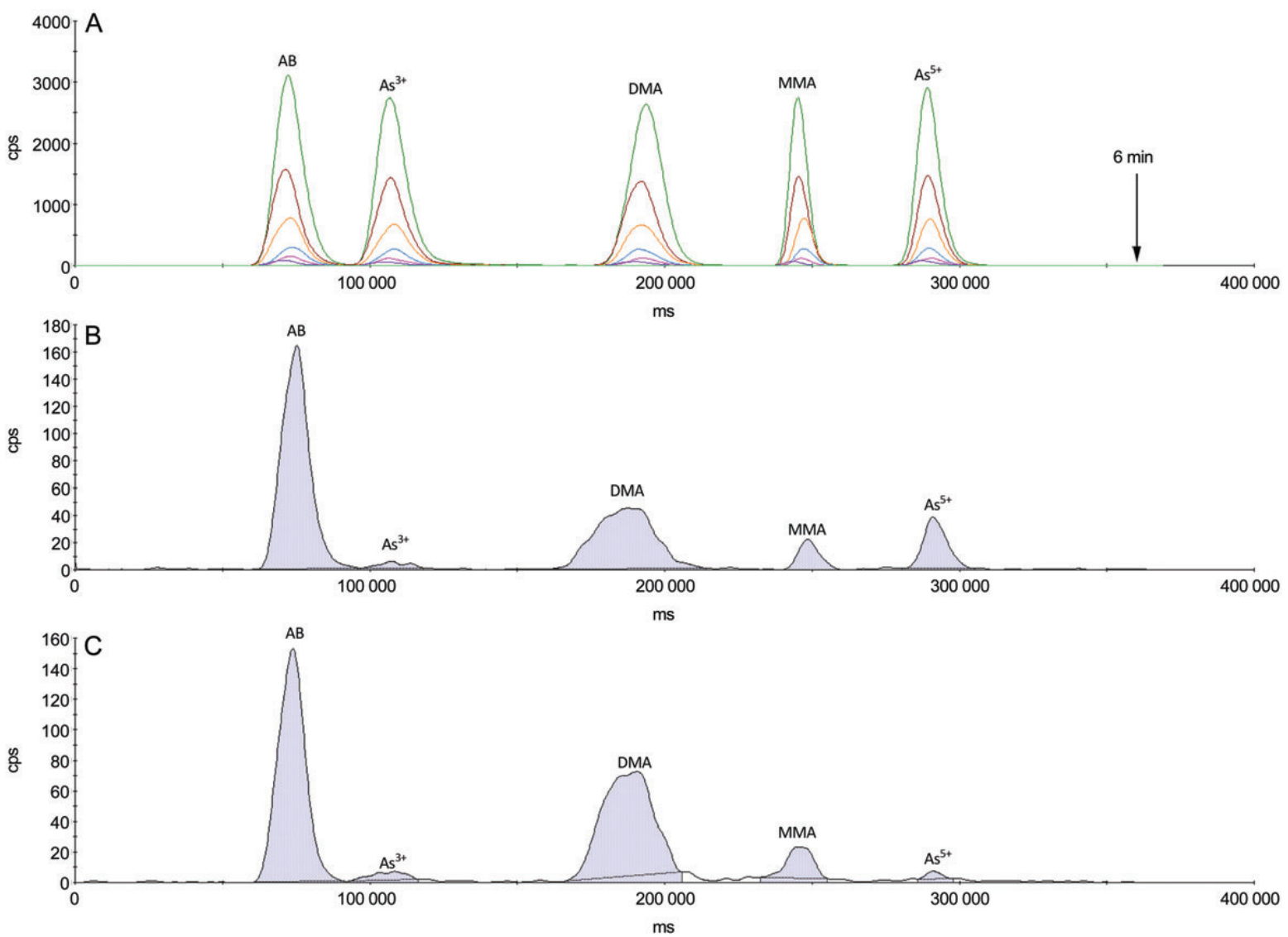

Figure 1. Chromatograms showing full separation of five arsenic species in $<6$ min using an ESI OneFAST system coupled to a 5-cm Dionex AG7 anion exchange column, with mobile phases 2 and $70 \mathrm{mM}$ of ammonium carbonate solution. (A) Overlay of the individual standard calibration chromatograms of $0-20 \mu \mathrm{g} / \mathrm{L}$ calibration. (B) CRM Clinchek 1 for arsenic species in urine. (C) A urine sample of an unexposed individual adult. 


\section{Study results}

Of the 95 volunteers who participated, 82 were nonsmokers ( 33 females and 49 males) and 13 were smokers ( 9 females and 4 males). The urine results are summarized in Table III; the median and the 95th percentile concentrations are expressed both uncorrected for creatinine $(\mu \mathrm{g} / \mathrm{L})$ and creatinine corrected $(\mu \mathrm{mol} / \mathrm{mol})$ in urine to adjust for urinary dilution. Creatinine concentration ranged between 0.89 and $26.45 \mathrm{mmol} / \mathrm{L}$. Nine individuals had a low (below $3 \mathrm{mmol} / \mathrm{L}$ ) or high (above $30 \mathrm{mmol} / \mathrm{L}$ ) creatinine; however, creatinine concentration was not an exclusion criterion (the reason being that excluding these individuals would exclude a much higher proportion of females than males). As $63 \%$ of $\mathrm{As}^{5+}$ measurements were below the LOQ, the median $\mathrm{As}^{5+}$ concentration could not be quantified. The 95th percentiles for $\mathrm{As}^{3+}$ and $\mathrm{As}^{5+}$ were 0.54 (0.99 $\mu \mathrm{mol} / \mathrm{mol}$ creatinine $)$ and $0.23 \mu \mathrm{g} / \mathrm{L}(0.35 \mu \mathrm{mol} / \mathrm{mol}$ creatinine), respectively. MMA, DMA and AB gave 95 th percentiles of $2.37(3.08 \mu \mathrm{mol} / \mathrm{mol}$ creatinine $), 12.68(16.08 \mu \mathrm{mol} / \mathrm{mol}$ creatinine) and $126.7 \mu \mathrm{g} / \mathrm{L}(174.7 \mu \mathrm{mol} / \mathrm{mol}$ creatinine $)$, respectively. The median and 95 th percentile concentrations for male and female urinary arsenic species are also presented in

\section{Table}

The background equivalent concentration (calibration curve intercept), LOD (signal-to-background noise) and limit of quantification for all the five species of arsenic in $\mu \mathrm{g} / \mathrm{L}$, in addition to the number and percentage of samples less than the LOO

\begin{tabular}{llllll}
$\begin{array}{l}\text { Arsenic } \\
\text { species }\end{array}$ & $\begin{array}{l}\mathrm{BEC} \\
(\mu \mathrm{g} / \mathrm{L})\end{array}$ & $\begin{array}{l}\mathrm{LOD} 3 \times \mathrm{SD} \text { of the } \\
\text { blank }(\mu \mathrm{g} / \mathrm{L})\end{array}$ & $\begin{array}{l}\mathrm{LO0} \\
(\mu \mathrm{g} / \mathrm{L})\end{array}$ & $\begin{array}{l}\text { No. of samples } \\
<\mathrm{L} 00\end{array}$ & $\begin{array}{l}\% \\
<\mathrm{LO0}\end{array}$ \\
\hline $\mathrm{As}^{3+}$ & 0.001 & 0.004 & 0.02 & 11 & 12 \\
$\mathrm{As}^{5+}$ & 0.003 & 0.051 & 0.04 & 60 & 63 \\
MMA & 0.002 & 0.030 & 0.04 & 2 & 2 \\
$\mathrm{DMA}$ & 0.003 & 0.003 & 0.04 & 0 & 0 \\
$\mathrm{AB}$ & 0.002 & 0.003 & 0.03 & 1 & 1 \\
\hline
\end{tabular}

Table III. Females were observed to have lower median and 95th percentiles of uncorrected for creatinine $(\mu \mathrm{g} / \mathrm{L})$ concentrations for arsenic species $\mathrm{As}^{3+}, \mathrm{As}^{5+}$ and MMA. However, for the creatinine-corrected data, females show a lower median concentration than males, but a higher 95 th percentile for $\mathrm{As}^{3+}$.

\section{Statistical results}

General statistical analysis using Prism Graphpad version 4 for Windows was performed, comparing each arsenic species in relation to gender and smoking habits. Using a two-tailed MannWhitney nonparametric statistical test, there was no significant difference between smokers and nonsmokers for inorganic arsenic. Furthermore, no significant difference was found between females and males for $\mathrm{As}^{3+}, \mathrm{As}^{5+}$, MMA or $\mathrm{AB}$; however, a statistically significant higher value for DMA was found in females in the creatinine-corrected values only. The most likely reason for this could be that women tend to have lower creatinine levels than men (23), resulting in a higher corrected DMA value for women than for men.

The Bayesian regression model was fitted separately for $\mathrm{As}^{3+}$, MMA, DMA and AB; however, due to the large proportion (63\%) of $\mathrm{As}^{5+}$ measurements below the LOQ, the analysis was not carried out for $\mathrm{As}^{5+}$. When the model was fitted to the data, a creatinine coefficient of one was highly plausible for $\mathrm{As}^{3+}, \mathrm{MMA}$, DMA and $\mathrm{AB}$ (i.e., the credible interval for the parameter $\beta_{1}$ for each of these species included unity). This indicated that a creatinine correction may be effective for these species. The model was subsequently refitted to the data with $\beta_{1}$ set to unity, which corresponds to fitting a regression model to creatininecorrected concentrations. The model considered all the variables including gender, smoking and dietary factors. Variables that were considered to be nonsignificant at the 5\% level were subsequently removed from the model.

\section{Table II}

Results obtained for five species of arsenic in urinary CRM and an external quality assurance scheme

\begin{tabular}{|c|c|c|c|c|c|c|c|c|c|}
\hline \multirow[t]{3}{*}{ Arsenic species } & \multirow[t]{3}{*}{$\begin{array}{l}\text { In-house spiked urine }(10 \mu \mathrm{g} / \mathrm{L}) \\
\% \text { recovery }(n=29)\end{array}$} & \multicolumn{2}{|c|}{$\begin{array}{l}\text { Clinchek Level 1-923 } \\
\text { (Cert As species } \mu \mathrm{g} / \mathrm{L} \text { ) }\end{array}$} & \multicolumn{2}{|c|}{$\begin{array}{l}\text { Clinchek Level 2-923 } \\
\text { (Cert As species } \mu \mathrm{g} / \mathrm{L} \text { ) }\end{array}$} & \multicolumn{4}{|c|}{$\begin{array}{l}\text { G-EQAS_-Round } \\
50(\mu \mathrm{g} / \mathrm{L})\end{array}$} \\
\hline & & \multirow[b]{2}{*}{ Target } & \multirow[b]{2}{*}{ Result } & \multirow[b]{2}{*}{ Target } & \multirow[b]{2}{*}{ Result } & \multicolumn{2}{|l|}{$50-2 A$} & \multicolumn{2}{|l|}{$50-2 B$} \\
\hline & & & & & & Target & Result & Target & Results \\
\hline $\begin{array}{l}\mathrm{As}^{3+} \\
\mathrm{As}^{5+} \\
\mathrm{MMA} \\
\mathrm{DMA} \\
\mathrm{AB}\end{array}$ & $\begin{array}{l}9.0 \pm 0.8(90 \%) \\
9.3 \pm 0.9(93 \%) \\
9.3 \pm 1.0(93 \%) \\
9.3 \pm 1.1(93 \%) \\
9.3 \pm 1.8(93 \%)\end{array}$ & $\begin{array}{l}1.44-3.84 \\
2.10-4.90 \\
1.50-3.50 \\
5.88-13.7 \\
12.6-21.0\end{array}$ & $\begin{array}{r}0.5 \pm 0.1 \\
4.2 \pm 0.3 \\
2.8 \pm 0.5 \\
9.5 \pm 0.5 \\
15.4 \pm 1.1\end{array}$ & $\begin{array}{l}7.05-11.8 \\
18.9-31.5 \\
5.03-8.38 \\
32.6-54.3 \\
23.0-34.6\end{array}$ & $\begin{array}{r}2.9 \pm 0.6 \\
30.5 \pm 1.8 \\
7.2 \pm 0.5 \\
43.6 \pm 1.8 \\
27.7 \pm 1.2\end{array}$ & $\begin{array}{l}1.7-4.1 \\
3.8-8.6 \\
1.0-2.8 \\
8.3-14.9 \\
\quad-\end{array}$ & $\begin{array}{r}2.2 \\
6.0 \\
1.7 \\
11.6 \\
-\end{array}$ & $\begin{array}{c}4.9-9.1 \\
13.5-24.3 \\
6.8-11.6 \\
64.8-96 \\
-\end{array}$ & $\begin{array}{r}8.1 \\
21.8 \\
10.9 \\
95.9 \\
-\end{array}$ \\
\hline
\end{tabular}

Table III

Statistical summary of median and 95th percentile concentrations for all the five species of arsenic in both $\mu \mathrm{g} / \mathrm{L}$ and $\mu \mathrm{mol} / \mathrm{mol}$ creatinine, for both total urine samples and female and male urine samples

\begin{tabular}{|c|c|c|c|c|c|c|c|c|c|c|}
\hline & \multicolumn{2}{|l|}{$\mathrm{As}^{3+}$} & \multicolumn{2}{|l|}{$\mathrm{As}^{5+}$} & \multicolumn{2}{|l|}{ MMA } & \multicolumn{2}{|l|}{ DMA } & \multicolumn{2}{|l|}{$A B$} \\
\hline & $\mu \mathrm{g} / \mathrm{L}$ & $\mu \mathrm{mol} / \mathrm{mol}$ creatinine & $\mu \mathrm{g} / \mathrm{L}$ & $\mu \mathrm{mol} / \mathrm{mol}$ creatinine & $\mu \mathrm{g} / \mathrm{L}$ & $\mu \mathrm{mol} / \mathrm{mol}$ creatinine & $\mu \mathrm{g} / \mathrm{L}$ & $\mu \mathrm{mol} / \mathrm{mol}$ creatinine & $\mu \mathrm{g} / \mathrm{L}$ & $\mu \mathrm{mol} / \mathrm{mol}$ creatinine \\
\hline \multicolumn{11}{|l|}{ Total: $n=95$} \\
\hline Median & 0.11 & 0.19 & $<\mathrm{LOO}$ & $<\mathrm{L} 00$ & 0.56 & 0.90 & 2.44 & 4.30 & 3.87 & 10.0 \\
\hline 95th Percentile & 0.54 & 0.99 & 0.23 & 0.35 & 2.37 & 3.08 & 12.68 & 16.08 & 126.7 & 174.7 \\
\hline \multicolumn{11}{|l|}{ Females: $n=42$} \\
\hline Median & 0.07 & 0.15 & $<\mathrm{LOO}$ & $<\mathrm{L} 00$ & 0.48 & 0.95 & 2.43 & 5.35 & 6.77 & 19.4 \\
\hline 95th percentile & 0.44 & 1.05 & 0.15 & 0.38 & 2.10 & 3.42 & 12.68 & 26.7 & 333.9 & 672.8 \\
\hline \multicolumn{11}{|l|}{ Male: $n=53$} \\
\hline Median & 0.15 & 0.22 & $<\mathrm{LOO}$ & $<\mathrm{LOO}$ & 0.57 & 0.90 & 2.44 & 4.10 & 3.42 & 6.10 \\
\hline 95th percentile & 0.6 & 0.97 & 0.31 & 0.97 & 2.67 & 2.69 & 12.59 & 11.1 & 101.5 & 129.4 \\
\hline
\end{tabular}


The results of the Bayesian model showed that there was no significant dietary difference between females and males. However, it did show a statistically significant difference between gender for arsenic species $\mathrm{AB}$ and DMA only. The mean urinary $\mathrm{AB}$ and $\mathrm{DMA}$ values for females were $\sim 77$ and $28 \%$ higher than males. In agreement with the nonparametric statistical test, the Bayesian model found no significant difference between smokers and nonsmokers; however, there were only 13 smokers (14\% of the study group) in the dataset. As expected, the consumption of fish resulted in increased urinary $\mathrm{AB}$ and DMA concentrations. Volunteers who had eaten fish had urinary DMA concentrations twice as high as that in those who had not eaten fish and $\mathrm{AB}$ concentrations 10 times higher than that in those who had not eaten fish. The Bayesian model also showed that the mean concentration for urinary MMA was twice as high for those who had consumed fish than that in those who had not. However, the consumption of shellfish did not have an effect on urinary MMA concentrations and showed only a $27 \%$ increase in urinary DMA concentrations and a 2 -fold increase in urinary $\mathrm{AB}$ concentrations. Red wine also had a statistically significant effect on urinary $\mathrm{AB}$; the consumption of red wine was found to increase $\mathrm{AB}$ concentrations by $79 \%$ even after adjusting for consumption of fish and shellfish (of the 31 individuals who had consumed red wine, nine had consumed neither fish nor shellfish). Other dietary factors such as rice, mushrooms, real ale, poultry, rice milk and bran were not found to be statistically significant in the model for any of the four arsenic species.

To quantify and compare the variation of lognormally distributed measurements between the different arsenic species, the geometric coefficient of variation (GCV) was determined. The GCV quantifies intrasubject variability and is calculated as:

$$
\mathrm{GCV}=\sqrt{e^{s^{2}}-1}
$$

where $s$ is the standard deviation of the data after a lognormal transformation. The greater the GCV, the greater the variability in the measurements. AB showed the greatest variability with a GCV of 2.96, and DMA showed the lowest variability with a GCV of 0.77 As $^{3+}$ and MMA had a GCV of 1.38 and 0.80 , respectively.

The correlation between species was determined by calculating Pearson's correlation coefficient (a measure of the linear association between two variables) for the natural logarithm of the pairs of species. Nondetects were dealt with by imputation, whereby their values were replaced with the corresponding central estimates from the MCMC analysis, rather than by a common fixed value. This ensured that the nondetects did not introduce an artificial pattern to the data at low concentrations that could potentially dominate the dataset (25). A strong positive correlation was found between MMA and DMA; a moderate correlation was found between $\mathrm{As}^{3+}$ and MMA, $\mathrm{As}^{3+}$ and DMA, MMA and $\mathrm{AB}$ as well as DMA and $\mathrm{AB}$. A very weak correlation was found between $\mathrm{As}^{3+}$ and $\mathrm{AB}$. The correlation coefficient was not calculated for $\mathrm{As}^{5+}$ due to the very high proportion (63\%) of measurements below the LOQ.

\section{Discussion}

Exposure to arsenic can occur through diet, environmental or occupational sources. Reported here is the determined urinary arsenic speciation concentrations collected from 95 volunteers who are not occupationally exposed to arsenic. The median uncorrected for creatinine $(\mu \mathrm{g} / \mathrm{L})$ concentrations for all the five species of arsenic gave good agreement with other studies where 'control' unexposed samples were reported and for background level studies $(14,15,19,21)$. Both the NHANES (14) and HBECC (15) studies quantified DMA and AB only, due to the large proportion of reported nondetects for $\mathrm{As}^{3+}, \mathrm{As}^{5+}$ and MMA. The LODs reported in the NHANES study were $1.2 \mu \mathrm{g} / \mathrm{L}$ for $\mathrm{As}^{5+}, 1 \mu \mathrm{g} / \mathrm{L}$ for $\mathrm{As}^{3+}, 0.9 \mu \mathrm{g} / \mathrm{L}$ for MMA, $1.7 \mu \mathrm{g} / \mathrm{L}$ for DMA and $0.4 \mu \mathrm{g} / \mathrm{L}$ for $\mathrm{AB}(26)$, and most of the 95 th percentiles reported in the HBECC study are marked as 'use with caution' or 'unreliable to publish' (15). It could be argued that both of these arsenic speciation methods are not suitable for the analysis of control samples. The LOD and LOQ values reported for this novel arsenic speciation method demonstrated in this study are lower and, therefore, better suited to the analysis of background samples. The LOQs reported in both Heitland and Koster (18) and Morton and Leese (20) could not achieve LOQ concentrations of $<0.1 \mu \mathrm{g} / \mathrm{L}$ for any of the five species of arsenic, whereas in this study the LOQ ranges from 0.02 to $0.04 \mu \mathrm{g} / \mathrm{L}$. Despite differing LOD and LOQ, the 95th percentile uncorrected for creatinine concentrations $(\mu \mathrm{g} / \mathrm{L})$ for all the arsenic species apart from $\mathrm{AB}$ gave very good agreement with those reported by both the NHANES (14) study for US unexposed residents and Heitland and Koster (18) for German unexposed residents $(n=98)$. The median and the 95th percentile for creatinineuncorrected concentration $(\mu \mathrm{g} / \mathrm{L})$ for total inorganic arsenic and methylated metabolites (not including $\mathrm{AB}$ in this study) are 3.1 and $15.1 \mu \mathrm{g} / \mathrm{L}$, respectively, which are both lower than the BEI (12) and BAT (13) values of 35 and $50 \mu \mathrm{g} / \mathrm{L}$, respectively. In this study, the 95 th percentile concentration for $\mathrm{AB}$ gave a much higher value of $126.7 \mu \mathrm{g} / \mathrm{L}(174.7 \mu \mathrm{mol} / \mathrm{mol}$ creatinine $)$, compared with 35.0 (13) and $22.7 \mu \mathrm{g} / \mathrm{L}$ (18) reported in other studies. However, the HBECC (15) study gave a slightly higher 95th percentiles of $4.5 \mu \mathrm{g} / \mathrm{L}$ for $\mathrm{As}^{3+}$ and $30 \mu \mathrm{g} / \mathrm{L}$ for DMA, although their reported value of $110 \mu \mathrm{g} / \mathrm{L}$ for $\mathrm{AB}$ is similar to that obtained in our study. The predominant dietary species of arsenic (AB) is found primarily in seafood, shellfish, algae and seaweed products; however, it can also be found in poultry (5) and cereal products such as rice and bran (6). AB is thought to be the end point of arsenic metabolism in marine animals. It is not, however, believed to be accumulative in humans, and following ingestion, $\mathrm{AB}$ is eliminated unchanged in urine, within a couple of days. However, a study by Newcombe et al. (7) reported that $\mathrm{AB}$ was present in the urine of volunteers after 12 days of an AB-free diet (no seafood or shellfish, chicken or mushrooms), however rice was consumed. Newcombe et al. (7) suggested that dietary arsenic (AB) is present in rice. In our study, 11 urine samples had AB present, when no fish, seafood or seaweed products had been eaten in the preceding 7 days, but red wine, poultry and rice had been consumed. The median and 95th percentile concentrations for DMA in this study are 2.44 $(4.30 \mu \mathrm{mol} / \mathrm{mol}$ creatinine $)$ and $12.68 \mu \mathrm{g} / \mathrm{L}(16.08 \mu \mathrm{mol} / \mathrm{mol}$ creatinine), respectively. The presence of methylated metabolites in urine samples, which show no exposure to inorganic arsenic, suggests that dietary sources are not only responsible for the presence of $\mathrm{AB}$ in urine, but also DMA and to a lesser extent MMA. Pearson et al., (8) also reported that methylated metabolites can be from dietary sources, showing that excretion of DMA in urine increased after eating American long-grain rice. 
Elevated DMA in urine after the consumption of different types of fish has also been reported (4).

The regression model allowed us to investigate whether creatinine correction to the uncorrected data is appropriate. The results indicated that creatinine correction was effective for each of the arsenic species, where regression modeling was carried out $\left(\mathrm{As}^{3+}, \mathrm{MMA}, \mathrm{DMA}\right.$ and $\left.\mathrm{AB}\right)$.

\section{Conclusion}

The arsenic speciation method described in this paper has been used to determine urinary arsenic speciation results in a cohort of nonoccupationally exposed volunteers in the UK. This speciation method demonstrates high sensitivity, enabling accurate determinations of five species of arsenic in control urinary samples. Despite this, $63 \%$ of samples had no As ${ }^{5+}$ present.

Statistical analysis indicated that creatinine correction was effective for each of the arsenic species, where regression modeling was carried out $\left(\mathrm{As}^{3+}, \mathrm{MMA}, \mathrm{DMA}\right.$ and $\left.\mathrm{AB}\right)$. Fish consumption was associated with higher levels of MMA, DMA and $\mathrm{AB}$, shellfish with higher levels of DMA and $\mathrm{AB}$ and red wine with higher levels of $\mathrm{AB}$.

It can be expected that dietary sources of arsenic are not only responsible for the presence of $\mathrm{AB}$, but also for DMA and possibly MMA. The existence of methylated metabolites in a urine sample does not necessarily equate to evidence of inorganic arsenic exposure and its subsequent methylation. Therefore, a method where an amalgamated value (total less $\mathrm{AB}$ ) is determined is not an accurate measurement of exposure. To make an inferred exposure assessment, a speciation result is required and this could be further improved by keeping a diet log. There is a need for a detailed characterization of arsenic content in foodstuffs and beverages to make a more informed assessment. It would also be useful to establish further occupational exposure levels based on speciation results.

The results from this study mean that 95 th percentile background reference ranges have been established for $\mathrm{As}^{3+}, \mathrm{As}^{5+}$, MMA, DMA and AB. This will allow better interpretation of any subsequent exposure.

\section{Acknowledgments}

The authors thank all the volunteers for participating in this study and Kate Jones from the Health and Safety Laboratory for her help and support during this project.

\section{Funding}

This publication and the work it describes were funded by the Health and Safety Executive (HSE). Its contents, including any opinions and/or conclusions expressed, are those of the authors alone and do not necessarily reflect HSE policy.

\section{References}

1. IARC. (2011) Agents Classified by the IARC Monographs, Vol. 1105. UK: IARC. World Health Organisation. http://monographs.iarc. $\mathrm{fr} / \mathrm{ENG} /$ Classification/index.php (11 June 2013, date last accessed).

2. Huang, Y.K., Huang, Y.L., Hsueh, Y.M., Yang, M.H., Wu, M.M., Hsu, L.I. et al. (2008) Arsenic exposure, urinary speciation, and the incidence of urothelial carcinoma: a twelve year follow up study. Cancer Causes and Control, 19, 829-839.

3. Saha, J.C., Dikshit, A.K., Bandyopadhyay, M. (1999) A review of arsenic poisoning and its effects on human health. Critical Reviews in Environmental Science and Technology, 29, 281-313.

4. Heinrich-Ramm, R., Mindt-Prufert, S., Szadkowski, D. (2002) Arsenic species excretion after controlled seafood consumption. Journal of Chromatography, 778, 263-273.

5. Pinel-Raffaitin, P., Le Hecho, I., Amouroux, D., Potin-Gautter, M. (2007) Distribution and fate of inorganic and organic arsenic species in landfill leachates and biogases. Environmental Science and Technology, 41, 4536-4541.

6. Cleland, B., Tsuchiya, A., Kalman, D.A., Dills, R., Burbacher, T.M., White, J.W. et al. (2009) Arsenic exposure within the Korean community (United States) based on dietary behaviour and arsenic levels in hair, urine, air and water. Environmental Health Perspectives 117, 632-638.

7. Newcombe, C., Raab, A., Williams, P.N., Deacon, C., Haris, P.I., Meharg, A.A. et al. (2010) Accumulation or production of arsenobetaine in human? Journal of Environmental Monitoring, 12, 832-837.

8. Pearson, G.P., Greenway, G.M., Brima, E.I., Harris, P.I. (2007) Rapid arsenic speciation using ion pair LC-ICPMS with a monolithic silica column reveals increased urinary DMA excretion after ingestion of rice. Journal of Analytical Atomic Spectrometry, 22, 361-369.

9. Sleijkovec, Z., Falnoga, I., Goessler, W., Van Elteren, J.T., Raml, R., Podgornik, H. et al. (2008) Analytical artefacts in the speciation of arsenic in clinical samples. Analytica Chimica Acta, 607, 83-91.

10. Ito, K., Palmer, C.D., Steuerwald, A.J., Parsons, P.J. (2010) Determination of five arsenic species in whole blood by liquid chromatography coupled with inductively coupled plasma mass spectrometry. Journal of Analytical Atomic Spectrometry, 25, $1334-1342$

11. Hasegawa, T., Ishise, J., Fukumoto, Y., Matsuura, H., Zhu, Y., Umemura, T. et al. (2007) Chemical speciation of arsenic species in human blood serum by liquid chromatography using phosphatidylcholine-coated ODS column with detection by ICP-MS. Bulletin of the Chemical Society of Japan, 80, 498-502.

12. American Conference of Governmental Industrial Hygienists. (2012) TLVs and BEIs: Threshold Limit Values for Chemical Substances and Physical Agents. USA: Signature Publications. www.acgih.org.

13. DFG German DRF Deutsche Forschungsgemeinschaft. (2012) List of MAK and BAT Values 2009. Germany: Wiley-VCH.

14. Centres for Disease Control and Prevention. (2009) Fourth National Report on Human Exposure to Environmental Chemicals. Centres for Disease Control and Prevention. http://www.cdc.gov/exposurereport/ (11 June 2013, date last accessed).

15. Health Canada. (2013) Second Report on Human Biomonitoring of Environmental Chemicals in Canada. Health Canada. www. healthcanada.gc.ca/biomonitoring (15 May 2013, date last accessed)

16. Saoudi, A., Zeghnoun, A., Bidondo, M.L., Garnier, R., Cirimele, V., Persoons, R. et al. (2012) Urinary arsenic levels in the French adult population: the French National Nutrition and Health Study, 20062007. Science of the Total Environment, 433, 206-215.

17. Lee, J.W., Lee, C.K., Moon, C.S., Choi, I.J., Lee, K.J., Yi, S.M. et al. (2012) Korea national survey for environmental pollutants in the human body 2008: heavy metals in the blood or urine of the Korean population. International Journal of Hygiene and Environmental Health, 215, 449-457.

18. Heitland, P., Koster, H.D. (2008) Fast determination of arsenic species and total arsenic in urine by HPLC-ICP-MS: concentration ranges for unexposed German inhabitants and clinical case studies. Journal of Analytical Toxicology, 32, 308-314.

19. Morton, J., Mason, H. (2006) Speciation of arsenic compounds in urine from occupationally unexposed and exposed persons in the UK using a routine LC-ICP-MS method. Journal of Analytical Toxicology, 30, 293-301.

20. Morton, J., Leese, E. (2011) Arsenic speciation in clinical samples urine analysis using fast micro-liquid chromatography ICP-MS Analytical and Bioanalytical Chemistry, 399, 1781-1788. 
21. Apostoli, P., Bartoli, D., Allesio, L., Buchet, J.P. (1999) Biological monitoring of occupational exposure to inorganic arsenic. Occupational and Environmental Medicine, 56, 825-832.

22. Torra, M., Auge, J.M., Ballell, B., Rodamilans, M., Casoliva, C., Corbella, J. (2004) Biological monitoring of environmental exposure to arsenic in urine samples from residents of the city of Barcelona, Spain, after selective extraction of the toxicologically relevant arsenic forms. Trace Elements and Electrolytes, 21, 248-251.

23. Cocker, J., Mason, H.J., Warren, N.D., Cotton, R.J. (2011) Creatinine adjustment of biological monitoring results. Occupational Medicine, 61, 349-353.
24. Lunn, D.J., Thomas, A., Best, N., Spiegelhalter, D. (2000) WinbugsBayesian modelling framework: concepts, structure and extensibility. Statistics and Computing, 10, 325-337.

25. Helsel, D. (2009) Much Ado about next to nothing: incorporating nondetects in science. The Annals of Occupational Hygiene, 54, 257-262.

26. Caldwell, K.L., Jones, R.L., Verdon, C.P., Jarrett, J.M., Caudill, S.P., Osterloh, J.D. (2009) Levels of urinary total and speciated arsenic in the US population: national health and nutrition examination survey 2003-2004. Journal of Exposure Science and Environmental Epidemiology, 19, 59-68. 


\section{Chapter 3.}

The Investigation of Unexpected Arsenic Compounds Observed in Routine Biological Monitoring Urinary Speciation Analysis

E. Leese, M. Clench, J. Morton, P.H.E. Gardiner and V.A. Carolan. Toxics., 2017, 5(2):

doi:10.3390/toxics5020012

Published - Creative Commons Re-use Open Access Licence

http://www.mdpi.com/2305-6304/5/2/12 
Article

\title{
The Investigation of Unexpected Arsenic Compounds Observed in Routine Biological Monitoring Urinary Speciation Analysis
}

\author{
Elizabeth Leese ${ }^{1,2, *}$, Malcolm Clench ${ }^{2}$, Jackie Morton ${ }^{1}$, Philip H.E. Gardiner ${ }^{2}$ and \\ Vikki A. Carolan ${ }^{2}$ \\ 1 Health and Safety Executive, Harpur Hill, Buxton, Derbyshire SK17 9JN, UK; jackie.morton@hsl.gsi.gov.uk \\ 2 Biomolecular Sciences Research Centre, Sheffield Hallam University, Howard Street, Sheffield S1 1WB, UK; \\ m.r.clench@shu.ac.uk (M.C.); p.h.gardiner@shu.ac.uk (P.H.E.G.); v.a.carolan@shu.ac.uk (V.A.C.) \\ * Correspondence: liz.leese@hsl.gsi.gov.uk; Tel.: +44-129-821-8434
}

Academic Editor: Wayne Briner

Received: 24 April 2017; Accepted: 18 May 2017; Published: 20 May 2017

\begin{abstract}
This study investigates the identity of two unexpected arsenic species found separately in a number of urine samples sent to the Health and Safety Executive's Health and Safety Laboratory for arsenic speciation (arsenobetaine, $\mathrm{AB}$; arsenite, $\mathrm{As}^{3+}$; arsenate, $\mathrm{As}^{5+}$; monomethylarsonic acid, $\mathrm{MMA}^{5+}$; and dimethylarsinic acid, $\mathrm{DMA}^{5+}$ ). Micro liquid chromatography coupled to inductively coupled plasma mass spectrometry ( $\mu \mathrm{LC}-\mathrm{ICP}-\mathrm{MS})$ and electrospray time of flight tandem mass spectrometry (ESI-QqTOF-MS/MS) were used to identify the two arsenic peaks by comparison to several characterized arsenicals: arsenocholine, AC; trimethyl arsine oxide, TMAO; dimethylarsenoacetate, DMAA; dimethylarsenoethanol, DMAE; thio-dimethylarsinate, thio-DMA; thio-dimethylarsenoacetate, thio-DMAA and thio-dimethylarsenoethanol, thio-DMAE. The results from both the ICP-MS and ESI-QqTOF-MS/MS investigations indicate that the unexpected arsenic species termed peak 1 was thio-DMA. While the unexpected arsenic species termed peak 2 has yet to be identified, this investigation shows that it was not AC, TMAO, DMAA, DMAE, thio-DMA, thio-DMAA or thio-DMAE. This study demonstrates the incidence of unexpected arsenic species in both routine and non-routine urine samples from both workers and hospital patients.
\end{abstract}

Keywords: arsenic; speciation; thioarsenical; thio-DMA; urine; exposure; ICP-MS; oxoarsenicals

\section{Introduction}

Identifying arsenic species in urine samples presents both an analytical and biological challenge, as the metabolism and biotransformation of arsenic compounds in humans is complex [1]. In part this is due to the fact that there is no exact agreed process for this human metabolic pathway of arsenic in the literature, with Hayakawa [2], Naranmandura [3] and Wang [4] all proposing slightly different biomethylation pathways. In 1945, the Challenger pathway [5] was first proposed in humans suggesting an initial reduction of arsenate to arsenite followed by steps of oxidation methylation and reduction methylation as follows: arsenate $>$ arsenite $>$ monomethylarsonic acid $>$ monomethylarsonous acid $>$ dimethylarsinic acid $>$ dimethylarsinous acid $\left(\mathrm{As}^{5+}>\mathrm{As}^{3+}>\mathrm{MMA}^{5+}\right.$ $>\mathrm{MMA}^{3+}>\mathrm{DMA}^{5+}>\mathrm{DMA}^{3+}$ ). More recent studies [2-4] have suggested that the pentavalent methylated species are not intermediates as presumed in the Challenger pathway but rather an end product of arsenic biotransformation. It has also been shown that the presence of the mammalian methyltransferase enzyme As3MT [6,7] (previously named Cyt19) [8] is crucial for arsenic metabolism, as it enables the transfer of the methyl donor S-adenosyl-L-methionine (SAM) in the presence of glutathione (GSH) [2,9]. It is suggested that the primary site of arsenic methylation in humans is the 
liver $[8,10]$, because the liver retains and accumulates arsenic [11] in addition to being a rich source of GSH [12].

In the general population, the primary source of arsenic exposure is through ingestion from dietary and water sources. It is also possible that workplace exposure could occur in industries such as semiconductors (using gallium arsenide and arsine gas) and glass making. Inorganic arsenic and the methylated metabolite DMA can be found in rice and rice products $[13,14]$. The most common non-rice based dietary arsenic compounds are arsenobetaine $[\mathrm{AB}]$, arsenocholine $[\mathrm{AC}]$, trimethylarsine oxide [TMAO] and arsenosugars. These dietary species are found in marine algae, squid, crustaceans, bivalves and fish $[15,16]$. Humans are unable to further metabolise $A B$ and $A C$ after ingestion [17-19], as a result of four stable carbon bonds making them quasi-inert [19]. Subsequently, $\mathrm{AB}$ and $\mathrm{AC}$ are excreted in the urine unchanged and assumed to be completely non-toxic [17-19]. Arsenosugars, unlike $\mathrm{AB}$ and $\mathrm{AC}$, are bio-accessible and can be transformed into other metabolites after human ingestion. These possible metabolites are $\mathrm{DMA}^{5+}$ (the major metabolite) and numerous DMA analogues: dimethylarsenoacetate [DMAA]; dimethylarsenoethanol [DMAE]; thio-dimethylarsenoacetate [thio-DMAA]; thio-dimethylarsenoethanol [thio-DMAE]; and thio-dimethylarsinate or dimethylthioarsinic acid [thio-DMA / DMTA]. These compounds are named dimethylarsinoyl compounds where the arsinoyl group represents either $\mathrm{As}=\mathrm{O}$ or $\mathrm{As}=\mathrm{S}$ and so are commonly termed oxo- and thioarsenicals [20]. Both monomethylated and di-thiolated forms such as dimethyldithioarsinic acid [dithio-DMA/DMDTA], monomethylthioarsonic acid [thio-MMA/MMTA] and monomethyldithioarsonic acid [dithio-MMA/MMDTA] have also been proposed [21-25].

The first study to report a thioarsenical metabolite in "natural" biological samples was from the urine of sheep that inhabit the beaches of North Ronaldsway (Orkney Island, Scotland). These sheep were unique in that they are chronically exposed to arsenosugars as a result of their diet of seaweed [26]. The study determined and confirmed the thioarsenical using anion exchange high performance liquid chromatography (HPLC) coupled to an inductively coupled plasma mass spectrometry (ICP-MS) and electrospray mass spectrometry (ES-MS) [26]. Studies published by Francesconi's research group $[15,27,28]$ have reported the almost complete biotransformation of an ingested synthetically prepared arsenosugar, resulting in the excretion of a mix of twelve thio- and oxoarsenicals in urine. The investigation of these twelve arsenicals was performed using HPLC-ICP-MS and HPLC-ES-MS, with anion, cation or reverse phase chromatography to ensure full separation of all the arsenicals.

In later studies, thio arsenicals were reported in human urine samples from population studies [29-31]. These groups recognized the importance of accurate identification and employed ES-MS in addition to HPLC-ICP-MS. It is already known that $\mathrm{DMA}^{5+}$ is the major human urinary metabolite following both inorganic arsenic exposure and dietary arsenosugar exposure [32]; the literature implies perhaps the same premise for thio-DMA and possibly other thio-arsenicals being metabolites following both inorganic exposure or dietary arsenosugar exposure. This will be of some concern as toxicological studies have shown thio-DMA to be genotoxic, cytotoxic and as toxic or more cytotoxic than arsenite in studies with rats [33,34], human bladder cells $[23,35,36]$ and human lung cells [37-39], with one study describing thio-DMA as "one of the most toxicologically potent arsenic species, relevant to arsenic induced carcinogenicity" [23].

Since 2010, the Health and Safety Executive's (HSE's) Health and Safety Laboratory, based in Buxton, England, has been using a sensitive, robust and reliable speciation method for determining five species of arsenic in urine (arsenobetaine $[\mathrm{AB}]$, arsenite $\left[\mathrm{As}^{3+}\right]$, arsenate $\left[\mathrm{As}^{5+}\right]$, monomethylarsonic acid $\left[\mathrm{MMA}^{5+}\right]$ and dimethylarsinic acid $\left[\mathrm{DMA}^{5+}\right]$ ) using a micro LC system coupled to an ICP-MS with a specialty anion exchange micro column. In this study, we investigate two different unexpected arsenic peaks observed in the analysis of urine samples from forensic cases, occupational exposure, hospital patients and as part of a background level study. The two unexpected peaks have been termed peak 1 and peak 2. Identification was facilitated using micro liquid chromatography coupled to ICP-MS ( $\mu \mathrm{LC}-\mathrm{ICP}-\mathrm{MS}$ ) and electrospray time of flight tandem mass spectrometry (ESI-Qq-TOF-MS/MS). 
Previous unidentified DMA analogues which include; DMAA, DMAE, thio-DMAA, thio-DMAE and thio-DMA have been reported in urine of specific population studies exposed to inorganic arsenic contaminated drinking water [29,31], populations exposed to excessive amounts of arsenosugars $[26,30,40]$ and in specific human dosing or ingestion studies $[15,27,28,41,42]$. However, this is the first study to report the observation of these similar or same arsenic compounds not from specific epidemiological populations, but routine biological monitoring urine samples. These samples are from a variety of industrial companies and geographical locations with no unifying commonalties as to the source of exposure to help aid either the identification of the arsenic species or with interpretation/feedback for customers. Based on an extensive review of the literature and on the availability of arsenic compounds, seven arsenicals (DMAA, DMAE, thio-DMAA, thio-DMAE, thio-DMA, TMAO and AC) were used to help determine the two unexpected arsenic peaks. These two arsenic peaks to date have not been observed at the same time in the same urine sample. Arsenic structures in addition to the structures of the seven arsenic compounds investigated in this study can be found in Figure S1 in the supplementary information.

\section{Materials and Methods}

\subsection{Standards and Reagents}

Arsenic speciation compounds sodium arsenite $\left(\mathrm{NaAsO}_{2}\right)$, sodium arsenate $\left(\mathrm{Na}_{2} \mathrm{HAsO}_{4} \cdot 7 \mathrm{H}_{2} \mathrm{O}\right)$ and sodium cacodylate (DMA; $\mathrm{Na}\left(\mathrm{CH}_{3}\right) 2 \mathrm{AsO}_{2} \cdot 3 \mathrm{H}_{2} \mathrm{O}$ ) were all from Fisher Scientific (Loughborough, UK). Disodium methyl arsenate $\left(99 \% \mathrm{MMA} ; \mathrm{CH}_{3} \mathrm{AsO}(\mathrm{OH})_{2}\right)$ was from ChemService (West Chester, PA, USA). Arsenobetaine (AB; $\mathrm{C}_{5} \mathrm{H}_{11} \mathrm{AsO}_{2}$ ) was from Sigma-Aldrich (Dorset, UK). Arsenocholine ( $\mathrm{AC} ; \mathrm{C}_{5} \mathrm{H}_{14} \mathrm{AsBrO}$ ) and trimethyl arsine oxide (TMAO; $\mathrm{C}_{3} \mathrm{H}_{9} \mathrm{AsO}$ ) were both from Argus Chemicals (Vernio, Italy). Analytical quantities of the oxoarsenicals and thioarsenicals dimethylarsenoacetate (DMAA), dimethylarsenoethanol (DMAE), thio-dimethylarsinate (thio-DMA; $\left.\left(\mathrm{CH}_{3}\right) 2 \mathrm{As}(\mathrm{S}) \mathrm{OH}\right)$, thio-dimethylarsenoacetate (thio-DMAA) and thio-dimethylarsenoethanol (thio-DMAE) were all synthesized by Kevin Francesconi's group at Karl-Franzens University Graz. Ammonium carbonate and hydrogen peroxide were from VWR (Leicestershire, UK). All water used was ultrapure deionized water $(18.2 \mathrm{M} \Omega \mathrm{hm} \mathrm{cm}$ ) from a Millipore system (Merck Millipore, Billerica, MA, USA).

Single standard stock solutions of $1000 \mathrm{mg} \cdot \mathrm{L}^{-1}$ of $\mathrm{AB}, \mathrm{As}^{3+}, \mathrm{As}^{5+}, \mathrm{DMA}, \mathrm{MMA}, \mathrm{AC}$ and TMAO were prepared in $1 \%(v / v)$ nitric acid, and stored at $4{ }^{\circ} \mathrm{C}$. The analytical grade quantities of DMAA, DMAE, thio-DMAA, thio-DMAE and thio-DMA were reconstituted with $1 \mathrm{~mL}$ of water. Individual aliquots of $200 \mu \mathrm{L}$ were stored at $-80^{\circ} \mathrm{C}$ until analysis.

\subsection{Instrumentation}

\subsubsection{Species Identification by $\mu \mathrm{LC}-\mathrm{ICP}-\mathrm{MS}$}

Separation was performed using a hyphenated $\mu$ LC system with an ICP-MS (XSERIES 2, Thermo Fisher Scientific, Hemel Hempstead, UK). The separation of arsenic species was achieved using a $5 \mathrm{~cm}$ anion exchange guard column, (Dionex IONPAC AG7 $4 \mathrm{~mm} \times 50 \mathrm{~mm}$ i.d., $10 \mu \mathrm{m}$, Thermo Fisher Scientific). The micro-flow delivery of sample and mobile phase was accomplished using an ESI OneFAST system (Elemental Scientific, Warrington, UK), which consists of a six port switching valve and $1 \mathrm{~mL}$ sample loop. The ICP-MS was operated in collision cell (CCT) mode using $7 \%$ hydrogen in helium (approximately $3.5 \mathrm{~mL} / \mathrm{min}$ ), RF power $1400 \mathrm{~W}$, using a Ni Xt skimmer and a Pt sample cones.

The delivery of mobile phases and sample onto the column was controlled by the ICP-MS peristaltic pump at a constant $0.2 \mathrm{~mL} / \mathrm{min}$. The $\mu \mathrm{LC}$-ICP-MS method used comprises of a step gradient using two mobile phases of $2 \mathrm{mM}$ ammonium carbonate (mobile phase A) and $70 \mathrm{mM}$ ammonium carbonate (mobile phase B). Total acquisition time is $360 \mathrm{~s}$ with a sample injection time of $10 \mathrm{~s}$. Mobile phase A is the carrier solution, $120 \mathrm{~s}$ after the injection of the sample, mobile phase B is injected for $120 \mathrm{~s}$. 


\subsubsection{Species Identification by ESI-QqTOF-MS/MS}

Molecular identification was performed using an electrospray ionization quadrupole-time of flight (ESI-QqTOF) mass spectrometer (QStar XL MS-MS, Applied Biosystems, Concord, ON, Canada) with a turbo ion-spray source. The MS/MS experiments were carried out in positive ion mode with a collision energy of $20 \mathrm{eV}$ using argon as the collision gas. Samples were injected separately into the ESI-QqTOF-MS using syringe infusions.

\subsection{Sample Collection}

All participating individuals were provided with informed consent, in accordance with HS(G) 167 [43]. All urine samples analyzed for arsenic speciation were received at the Health and Safety Laboratory as part of the analytical service for biological monitoring to assess arsenic exposure. From 2012 to 2016, an unexpected peak was observed in $4 \%$ of urine samples analyzed. The urine samples arrive with little contextual data apart from name, date of birth and details of the sender. Urine samples containing an unidentified arsenic peak were from UK semi-conductor workers, South African miners, forensic incidents, hospital patients and a participant of an arsenic-unexposed background level study [44]. It is important to note that urine samples from South African Miners and hospital patients were only received at the Health and Safety Laboratory for arsenic speciation analysis if total arsenic concentrations exceeded the US biological exposure index (BEI) [45] of $35 \mu \mathrm{g} \cdot \mathrm{L}^{-1}$ (approximately $40 \mu \mathrm{mol} / \mathrm{mol}$ creatinine) at other laboratories. Upon receipt at the laboratory all urine samples for arsenic speciation are stored frozen at approximately $-20^{\circ} \mathrm{C}$ until analysis. For this study, samples that contained an unexpected peak had $1 \mathrm{~mL}$ aliquots taken and stored at $-80^{\circ} \mathrm{C}$ until identification analysis. Urine samples are not filtered prior to analysis and sample preparation involves only a 15-fold dilution with mobile phase A.

\section{Results}

\subsection{Identification of Unexpected Arsenic Peaks by $\mu L C-I C P-M S$}

During analysis of urinary arsenic speciation, both peak 1 and peak 2 eluted at different retention times to the five arsenic species routinely determined at the Health and Safety Laboratory $\left(\mathrm{AB}, \mathrm{As}^{3+}\right.$, $\mathrm{As}^{5+}, \mathrm{DMA}$ and MMA) (Figure 1A). Figure 1 shows that peak 1 (Figure 1B) is eluted between DMA and MMA at a retention time of $216 \mathrm{~s}$, peak 2 (Figure 1C) is eluted between MMA and $\mathrm{As}^{5+}$ at a retention time of $240 \mathrm{~s}$.

The analytical quantities of DMAA, DMAE, thio-DMAA, thio-DMAE, thio-DMA at approximate concentrations of $20-100 \mu \mathrm{g} \cdot \mathrm{L}^{-1}$, and $5 \mu \mathrm{g} \cdot \mathrm{L}^{-1}$ standards of AC and TMAO were injected onto the Dionex AG7 column. It was possible to observe each of the seven additional arsenic compounds with the existing $\mu$ LC-ICP-MS method, although not all species would be fully separated if injected together. Figure S2 in the supplementary information shows the elution retention times of each of these six oxoand thioarsenicals and $\mathrm{AC}$ when compared to the original five arsenic species. 

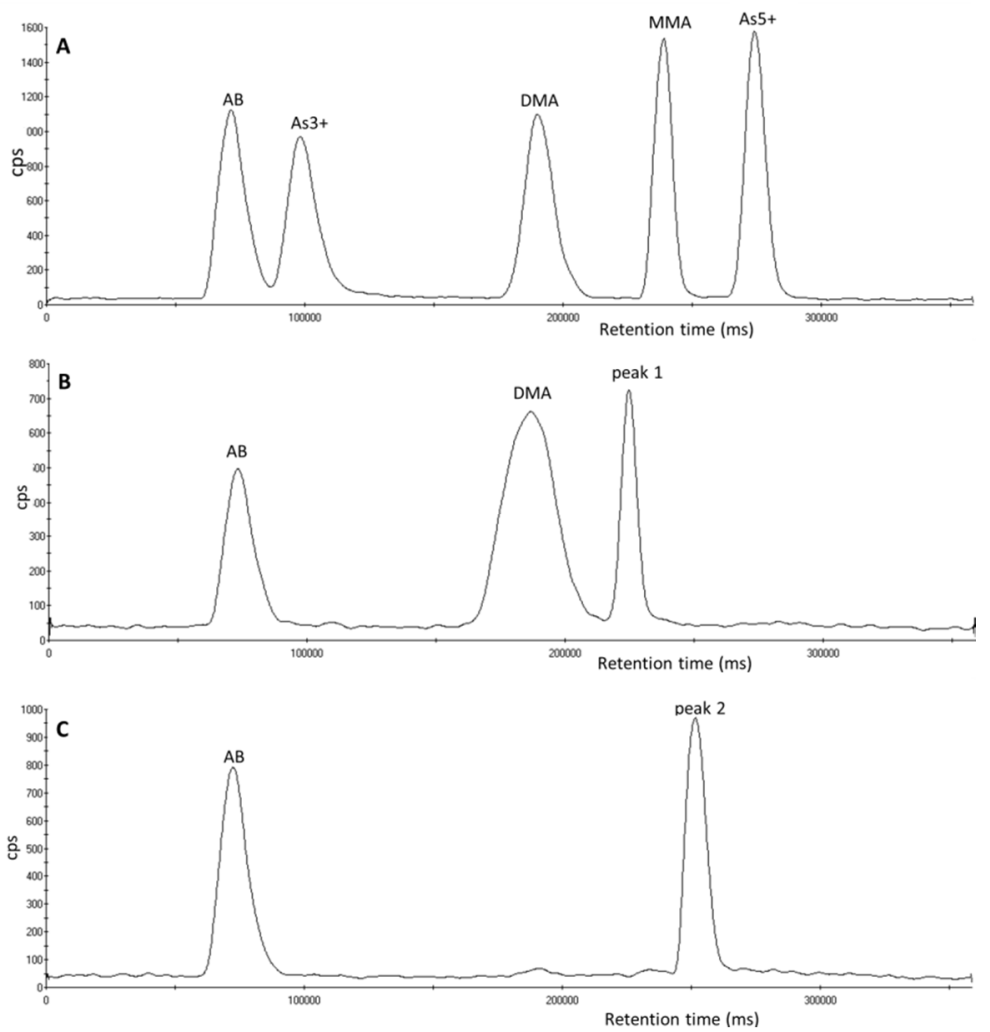

Figure 1. Chromatograms of arsenic species analyzed using an ESI OneFAST system coupled to a Dionex AG7 anion exchange column and ICP-MS using mobile phases $2 \mathrm{mM}$ and $70 \mathrm{mM}$ ammonium carbonate solution (A) A $5 \mu \mathrm{g} \mathrm{L}^{-1}$ standard of the five arsenic species. (B) A urine sample containing peak 1. (C) A urine sample containing peak 2.

\subsection{Investigation of Peak 1 by $\mu L C-I C P-M S$}

The urine samples analyzed where the arsenic peak, termed peak 1 was observed came from four different groups of individuals. The first group consisted of urine samples from two separate forensic cases, one of which was from a fatal arsenic poisoning where the approximate total arsenic concentration was $4800 \mu \mathrm{g} \cdot \mathrm{L}^{-1}(-11,500 \mu \mathrm{mol}$ mol creatinine) and contained the highest concentration of peak 1 in this study (approximate concentration $2800 \mu \mathrm{g} \cdot \mathrm{L}^{-1}$ ). The second group consisted of occupational samples from South African miners, where $33 \%$ of urine samples analyzed contained peak 1 . The third group were from hospital patients within the UK, where the presence of peak 1 accounts for $21 \%$ of all hospital patient samples analyzed, and the fourth group was a volunteer who participated in a study to determine unexposed background reference values for five arsenic species in a UK population. The approximate concentration of peak 1 in other urine samples presented here were all less than $50 \mu \mathrm{g} \cdot \mathrm{L}^{-1}$.

The urine samples that contained peak 1 varied in $\mathrm{pH}$ from $\mathrm{pH} 4-\mathrm{pH}$ 7.2. These differences in $\mathrm{pH}$ of the urine samples did not affect the retention time of peak 1 . The chromatogram of a urine sample containing peak 1 is shown in Figure 2B. When a urine sample containing peak 1 was spiked with a $20 \mu \mathrm{g} \cdot \mathrm{L}^{-1}$ solution containing the standard five species, peak 1 eluted separately from the other five arsenic species, however as shown in Figure 2A, peak 1 elutes on the shoulder of the MMA peak. Of the six oxo- and thioarsenicals (DMAA, DMAE, thio-DMA, thio-DMAA and thio-DMAE) and AC injected onto the column, thio-DMA and $\mathrm{AC}$ were the only species that had a similar retention time to peak 1 (see Figure 2B,D,E). When the urine sample containing peak 1 was spiked with thio-DMA at an approximate concentration of $90 \mu \mathrm{g} \cdot \mathrm{L}^{-1}$, the peak at the retention time of peak 1 increases (see Figure 2C). 

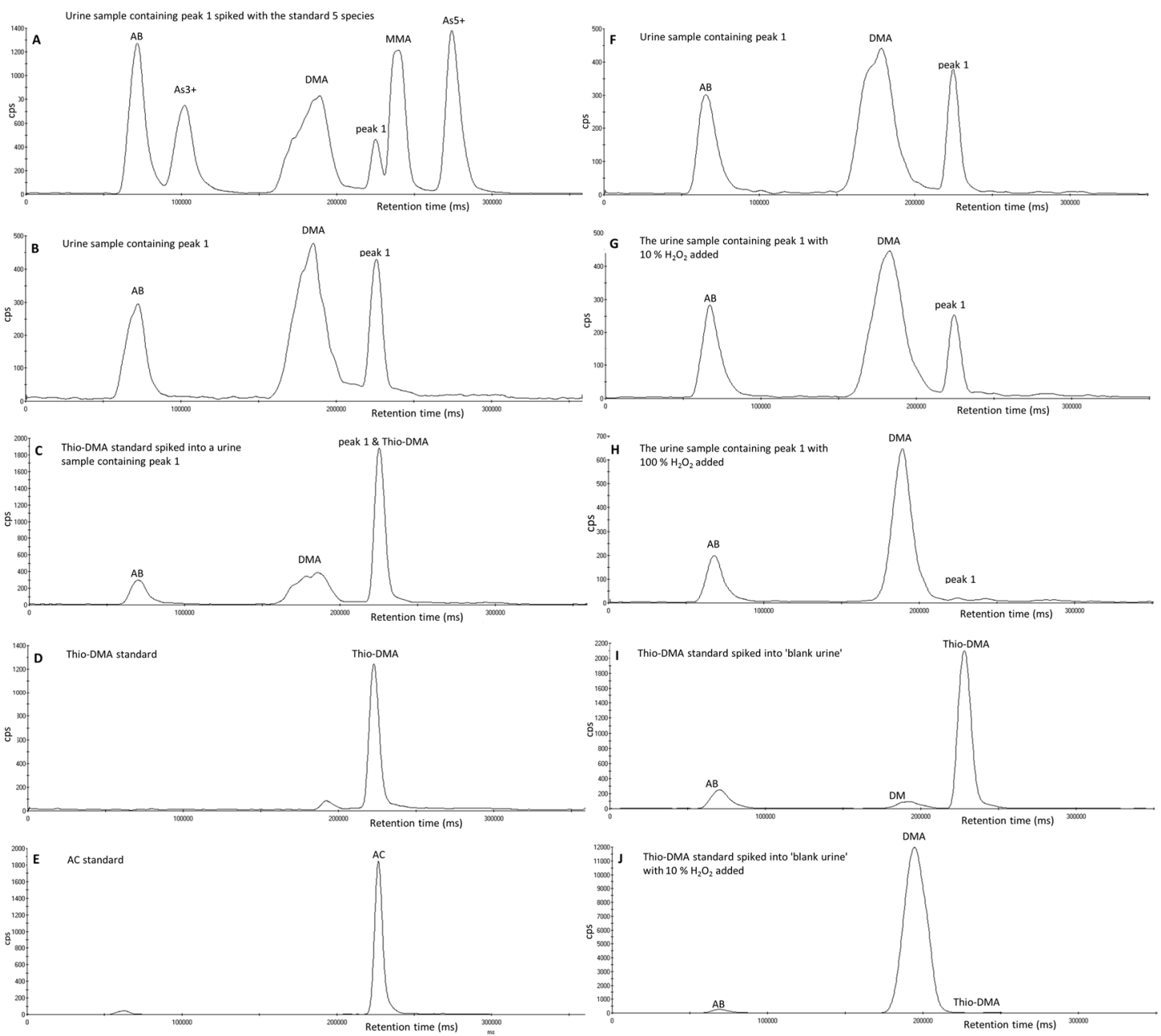

Figure 2. Chromatograms of arsenic species analyzed using an ESI OneFAST system coupled to a Dionex AG7 anion exchange column and ICP-MS using mobile phases $2 \mathrm{mM}$ and $70 \mathrm{mM}$ ammonium carbonate solution. (A) A urine sample containing peak 1 spiked with a $20 \mu \mathrm{g} \cdot \mathrm{L}^{-1}$ solution of the standard five species. (B) An unspiked urine sample containing peak 1. (C) The urine sample containing peak 1 spiked with an approximate concentration of $40 \mu \mathrm{g} \cdot \mathrm{L}^{-1}$ thio-DMA standard. (D) A thio-DMA standard at an approximate concentration of $40 \mu \mathrm{g} \cdot \mathrm{L}^{-1}$ and (E) A $5 \mu \mathrm{g} \cdot \mathrm{L}^{-1}$ AC standard. (F) The urine sample containing peak 1 . (G) The urine sample with $10 \% v / v \mathrm{H} 2 \mathrm{O} 2$ added (H) The urine sample with $100 \% \mathrm{H}_{2} \mathrm{O}_{2}$ added (I) Thio-DMA standard spiked into 'blank urine' not containing peak 1 (J) The spiked urine sample from chromatogram $\mathrm{D}$ with $10 \% v / v \mathrm{H}_{2} \mathrm{O}_{2}$ added.

When $10 \% v / v$ of $\mathrm{H}_{2} \mathrm{O}_{2}$ was added to a urine sample containing peak 1 , the height of peak 1 reduced while the DMA peak height increased (see Figure 2F,G). When this was repeated with $100 \%$ of $\mathrm{H}_{2} \mathrm{O}_{2}$, peak 1 completely disappeared and the DMA peak increased accordingly (see chromatogram $\mathrm{H}$ of Figure 2). When thio-DMA was treated with $10 \% v / v \mathrm{H}_{2} \mathrm{O}_{2}$, the thio-DMA peak was completely converted to a peak at the same retention time as DMA (see Figure 2I,J). When AC was treated with $100 \%$ of $\mathrm{H}_{2} \mathrm{O}_{2}$ the $\mathrm{AC}$ peak did not change retention time or reduce in peak height nor was there an effect on any of the other peaks. Therefore, this is evidence that peak 1 is not AC.

Interestingly, one particular urine sample containing peak 1 received from a hospital patient also showed the presence of an arsenic compound that eluted post column and was observed as a carryover peak following the injection of mobile phase $B$ in the following sample. A repeat injection of the urine sample followed by the injection of a mobile phase A blank sample showed the presence of the same peak as shown in Figure 3. Unfortunately, further investigation of this peak was prohibited due to 
conversion of this peak to peak 1 and DMA during weekend storage in the fridge at $4{ }^{\circ} \mathrm{C}$. The addition of $100 \% \mathrm{H}_{2} \mathrm{O}_{2}$ converted the very small remainder of this parent compound and all of peak 1 to DMA.
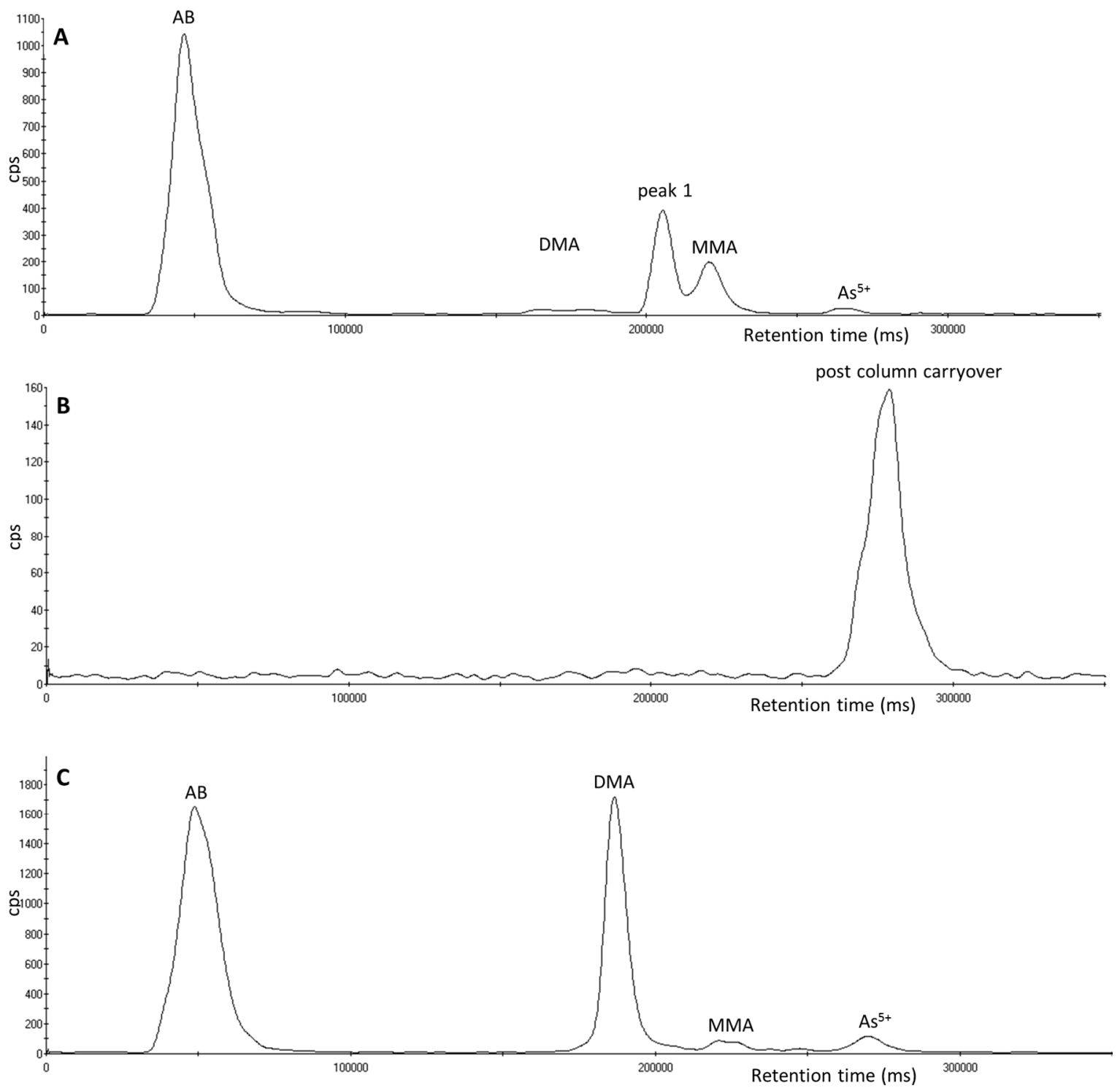

Figure 3. Chromatograms of arsenic species using an ESI OneFAST system coupled to a Dionex AG7 anion exchange column and ICP-MS using mobile phases $2 \mathrm{mM}$ and $70 \mathrm{mM}$ ammonium carbonate solution. (A) A urine sample from a hospital patient containing peak 1. (B) The post column carryover peak of an unknown into the blank sample immediately after the urine sample containing peak 1 . (C) Chromatogram of the 5-day-old urine sample with $100 \% \mathrm{H}_{2} \mathrm{O}_{2}$ added.

\subsection{Investigation of Peak 1 by ESI-QqTOF-MS/MS}

AC was not thought to be the identity of peak 1 because the arsenic compound termed peak 1 , was affected by the addition of $\mathrm{H}_{2} \mathrm{O}_{2}$ and $\mathrm{AC}$ was not. Early investigations using tandem mass spectrometry (not cited in the methodology) showed that when monitoring the protonated molecule $[\mathrm{M}+\mathrm{H}]^{+} \mathrm{m} / z 165$ of $\mathrm{AC}$, no signal was observed at the retention time of an AC standard, when the urine sample containing approximately $2800 \mu \mathrm{g} \cdot \mathrm{L}^{-1}$ of peak 1 was injected into the MS/MS.

While the $\mu$ LC-ICP-MS investigations of peak 1 had indicated that this peak was thio-DMA, further confirmation was sought using mass spectrometry. As shown in Figure 4, the $[\mathrm{M}+\mathrm{H}]^{+}$ion of thio-DMA is $m / z$ 155, with expected product ions of $m / z 137,121,109,107$ and 75. 
Since it was not possible in our laboratory to simultaneously analyze the arsenic species by coupling $\mu$ LC-ICP-MS with ESI-QqTOF-MS/MS, samples had to be analyzed separately using ESI-QqTOF-MS/MS. The samples were injected into the ESI-QqTOF-MS/MS using syringe infusion. To undertake this, an aqueous standard solution of thio-DMA of approximate concentration of $1600 \mu \mathrm{g} \cdot \mathrm{L}^{-1}$ was injected into the ESI-QqTOF-MS/MS (see Figure 4A). In a product ion scan of $m / z 155$, signals at $m / z 155,137$ and 109 were observed, and additionally a small product ion at $m / z 107$. Other minor peaks that were observed in the spectra of the thio-DMA standard were $\mathrm{m} / \mathrm{z} 127,113$, 99 and 95. In addition, a urine sample containing peak 1 has been fraction collected using the LC system on the ICP-MS and the fraction containing peak 1 was injected into the ESI-QqTOF-MS/MS (see Figure 4B). Again, the product ion mass spectrum of $m / z$ 155, gave peaks at $m / z 155,137,109$ corresponding to the product ion spectrum of the thio-DMA standard. The dominant peak was $\mathrm{m} / \mathrm{z} 95$ with intermediate peaks at $m / z$ 137, 113 and 109 and with smaller peaks at $m / z 155$ and 127, all of which concurred with the spectra of the thio-DMA standard.
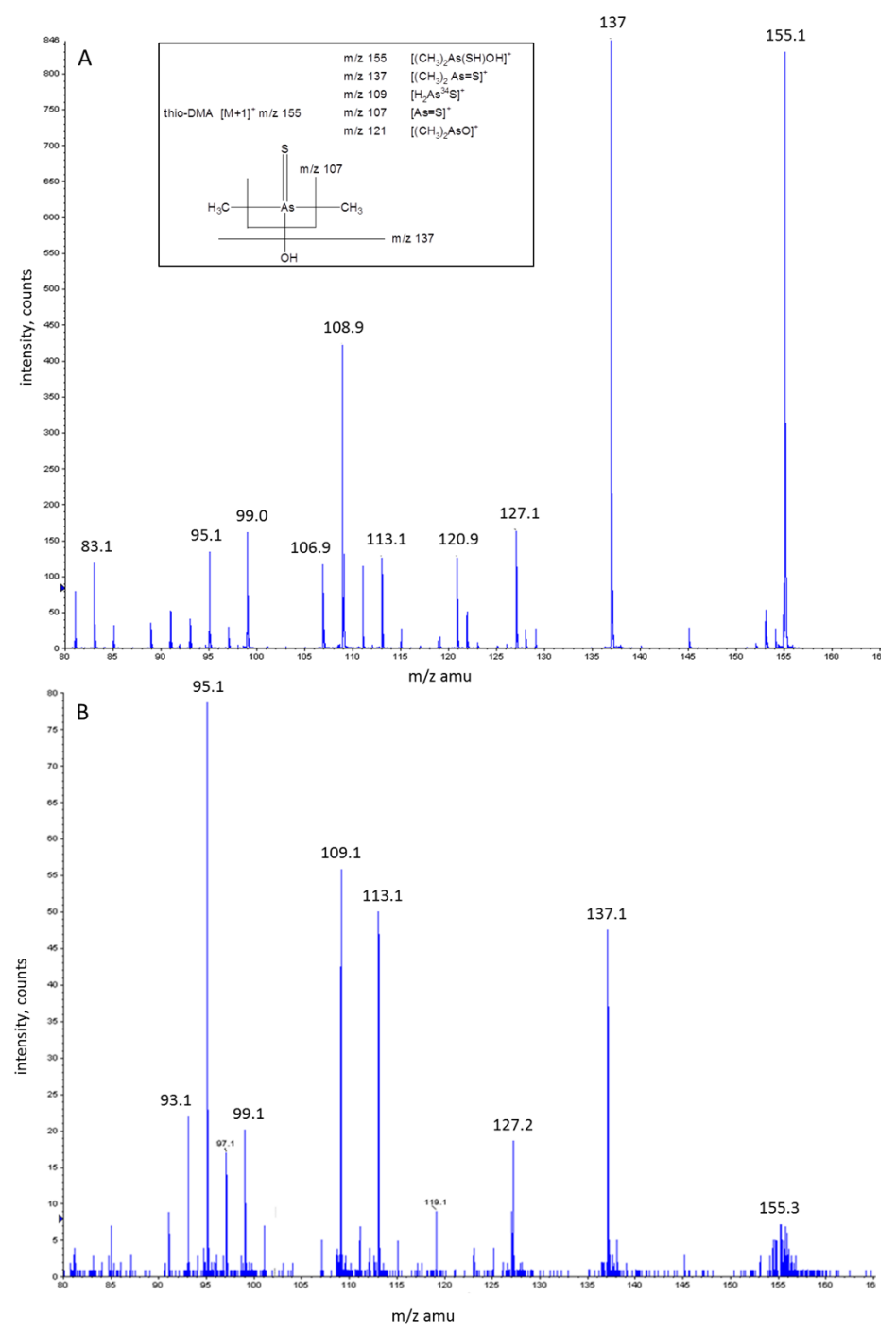

Figure 4. The chemical structure of thio-DMA, with suggested fragment ions when measured in positive ion mode by ESI-QqTOF-MS/MS within the two ESI-QqTOF-MS/MS spectra from product ion scans of $m / z$ 155, when infusing (A) Thio-DMA standard at an approximate concentration of $1600 \mu \mathrm{g} \cdot \mathrm{L}^{-1}$. (B) Fraction of a urine sample containing peak 1 with blank urine correction from a urine sample not containing peak 1 . 


\subsection{Investigation of Peak 2 by $\mu L C-I C P-M S$}

The urine samples containing the unexpected arsenic peak termed peak 2 came from two different groups of individuals. The first group were from different hospital patients, in separate hospitals within the UK. The symptoms of both individuals varied but both were surmised to have ingested an excessive amount of herbal remedies purchased online. The two individuals had consumed a different herbal remedy, one was kelp powder, administered orally by adding to food, the ingredient of the second herbal remedy was unknown, but was a black colored tablet administered orally. In total peak 2 has been observed in $9 \%$ of all hospital samples analyzed. The second group consisted of occupational urine samples from UK semi-conductor workers, peak 2 has been observed in $1 \%$ of all urine samples from semi-conductor workers.

The urine samples that contained peak 2 varied in $\mathrm{pH}$, from $\mathrm{pH}$ 5.5-pH 7.8. These differences in $\mathrm{pH}$ of the urine samples did not affect the retention time of peak 2. When a urine sample containing peak 2 was spiked with a $20 \mu \mathrm{g} \cdot \mathrm{L}^{-1}$ five species standard mix of arsenic species, peak 2 eluted separately from the other five arsenic species (see Figure 5A). Of the six oxo- and thioarsenicals (DMAA, DMAE, thio-DMA, thio-DMAA and thio-DMAE, TMAO) and AC injected onto the column, none matched the retention time of peak 2; however thio-DMAA is the closest. When a urine sample containing peak 2 was spiked with a standard of thio-DMAA, although not fully separated, two unresolved peaks were observed (see Figure 5B).
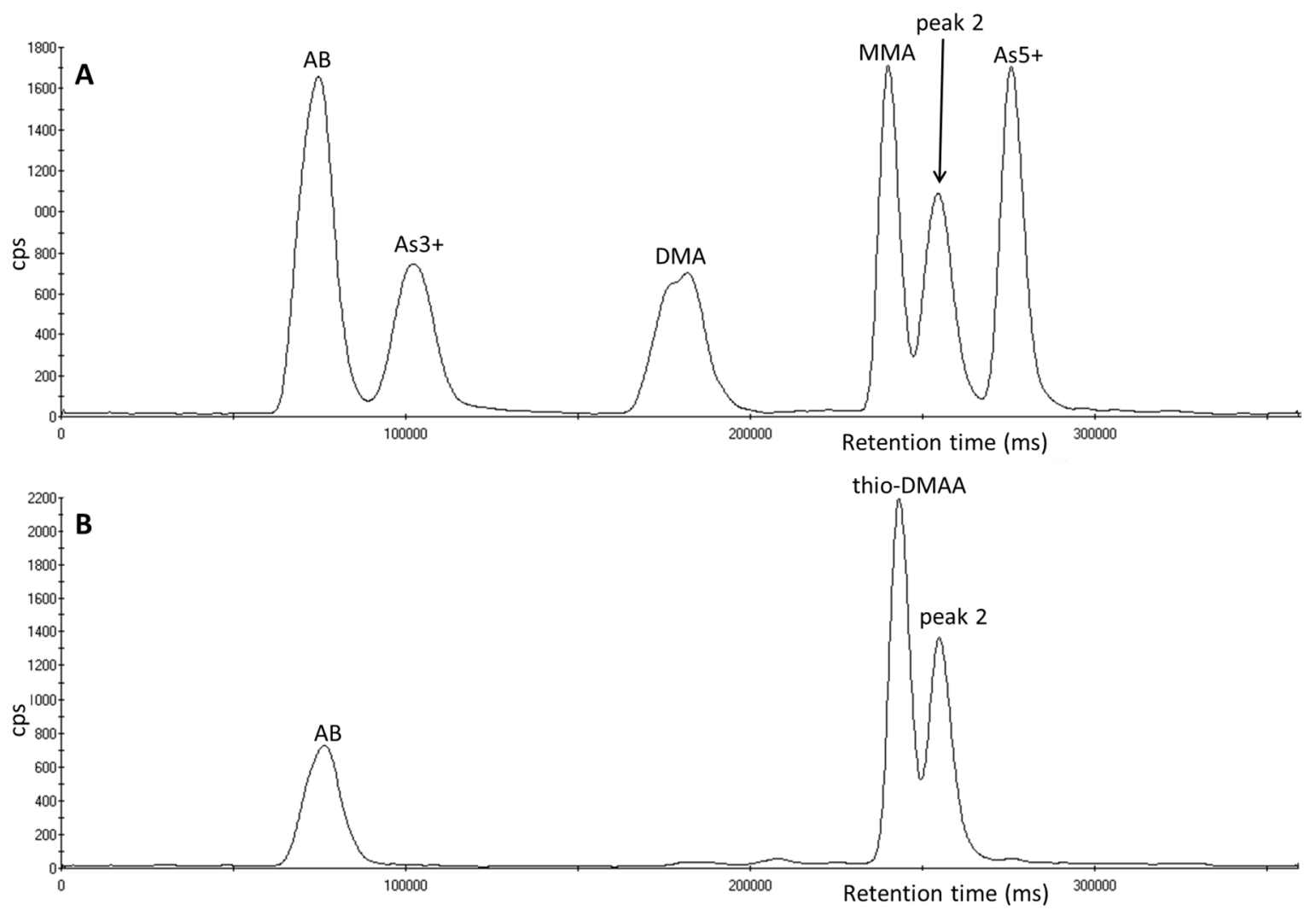

Figure 5. Chromatograms of arsenic species analyzed using an ESI OneFAST system coupled to a Dionex AG7 anion exchange column and ICP-MS using mobile phases $2 \mathrm{mM}$ and $70 \mathrm{mM}$ ammonium carbonate solution. (A) Chromatogram of a urine sample containing peak 2 spiked with a $20 \mu \mathrm{g} \cdot \mathrm{L}^{-1}$ standard mix of the five species of arsenic. (B) Chromatogram of the urine sample spiked with thio-DMAA.

When thio-DMAA was treated with $50 \% v / v \mathrm{H}_{2} \mathrm{O}_{2}$, the thio-DMAA peak was mostly converted to a peak at the same retention time as DMAA, leaving a much reduced sized peak of thio-DMAA. 
However when a urine sample containing peak 2 is treated with $100 \% \mathrm{H}_{2} \mathrm{O}_{2}$, the peak does not reduce or change in retention time and there is not a peak at the location assigned to DMAA. Chromatograms are shown in Figure S3 in the supplementary information.

\subsection{Investigation of Peak 2 by ESI-QqTOF-MS/MS}

Considerable efforts were made to analyze the urine samples with peak 2 by ESI-QqTOF-MS/MS. Two different tandem mass spectrometry instruments were used, different solvents were investigated and the fraction-collected peaks were freeze dried and reconstituted in an attempt to concentrate the samples. However, due to the inherent problems surrounding the difficulty in ionizing oxo- and thioarsenicals by MS-MS, there was insufficient sensitivity using the ESI-QqTOF-MS/MS to identify the protonated $[\mathrm{M}+\mathrm{H}]^{+}$ion of thio-DMAA at $m / z$ 197, or its expected product ions of $m / z 165,151$, 137, 107 and 75. Using negative ion mode was also unsuccessful. No definitive signal was observed across the mass range. The approximate concentration of the arsenic compound of peak 2 in all the urine samples used in this study was considered too low (estimated concentrations $<50 \mu \mathrm{g} \cdot \mathrm{L}^{-1}$ ) to detect any $[\mathrm{M}+\mathrm{H}]^{+}$or product ions which corresponded to DMAA, DMAE, thio-DMAA, thio-DMAE or thio-DMA. To aid identification of peak 2, no other analytical techniques were investigated as it was deemed that the ESI-QqTOF-MS/MS would have the lowest LOD to facilitate the identification of arsenic compounds at such low concentrations.

To date we have been unable to identify peak 2 , however this arsenic compound seems stable in urine, and to date has not converted to another peak when stored for 12 months frozen at either $-80{ }^{\circ} \mathrm{C}$ or $-20^{\circ} \mathrm{C}$.

\section{Discussion}

The investigation, by both $\mu \mathrm{LC}-\mathrm{ICP}-\mathrm{MS}$ and ESI-QqTOF-MS/MS, has determined that peak 1 is thio-DMA. Unfortunately, peak 2 has not been identified, although it has been possible to eliminate seven arsenic species in the process. This is not the only study to report unsuccessful identification of arsenic peaks in similar investigations $[15,27,28,41,43,46]$. While a human dosing study reported the almost complete biotransformation of an ingested synthetically prepared arsenosugar (2,3-dihydroxypropyl 5-deoxy-5-dimethylarsinoyl- $\beta$-D-riboside), which resulted in the excretion of twelve arsenicals in urine $[15,27,28]$, initially only three of the twelve could be identified [15], which were $\mathrm{DMA}^{5+}$, TMAO and DMAE. Repeat studies further identified DMAA, thio-DMAA, thio-DMAE, the arsenosugar and thio-arsenosugar and trace amounts of thio-DMA and TMAS, with two species remained unidentified $[27,28]$. All information in this challenging analytical field is valuable and contributes to the scientific knowledge and future studies of a similar nature.

The urine samples which contained thio-DMA (peak 1) were obtained from four sources consisting of two separate forensic cases, occupational exposure samples, a hospital patient and an individual participating in a background levels study. Only the urine samples from the two forensic cases had clear evidence of inorganic arsenic exposure (inorganic arsenic and elevated $\mathrm{DMA}^{5+}$ and $\mathrm{MMA}^{5+}$ were observed during speciation analysis). The urine sample of the additional hospital patient saw the elution of a further arsenic compound eluting post column in the following sample (Figure 3). This compound is potentially a "parent" arsenic compound and it was found to be quite unstable, as it had completely converted to thio-DMA after refrigeration at $4{ }^{\circ} \mathrm{C}$ and subsequently $\mathrm{DMA}^{5+}$ after the addition of hydrogen peroxide. The presence of this parent compound has not been observed previously or since, nor has it been observed in urine in the current literature to provide an identification suggestion. It is common knowledge in the literature $[23,25,26,30,38,47]$ that treatment of $\mathrm{DMA}^{5+}$ with hydrogen sulphide will produce thio-DMA. Furthermore, it is known that continued treatment of thio-DMA with hydrogen sulphide will produce dithio-DMA $[47,48]$ and the subsequent addition of hydrogen peroxide can convert dithio-DMA back to thio-DMA [49]. The study by Conklin [49] found the conversion between DMA, thio-DMA and dithio-DMA in aqueous standards was $\mathrm{pH}$ specific, with DMA only converting to its thio-analogues when the $\mathrm{pH}$ range was between $\mathrm{pH} 5-7$. Unfortunately, in 
our study, due to the rapid degradation of this observed parent compound in the urine sample and the lack of a dithio-DMA standard to match retention time, the authors were unable to make any initial analytical confirmation of the identity of the compound and can therefore only postulate the parent compound's identity as dithio-DMA.

It is possible that the source of peak 1 in the urine samples from the South African miners is either from dietary or occupational sources. However, in these samples inorganic arsenic was not observed, with the dominant species being $\mathrm{DMA}^{5+}$ and $\mathrm{MMA}^{5+}$ with smaller concentrations of $\mathrm{AB}$. These mixed findings of both inorganic exposure and dietary arsenosugar exposure are similar to those found in the literature. A study looking at women in Bangladesh exposed to inorganic arsenic in their drinking water found that $44 \%$ of urine samples from 75 women contained thio-DMA [31]. A similar study looking at a population exposed to inorganic arsenic through drinking water in West Bengal found thio-DMA in both urine and nails [29]. A noteworthy study by Raml [30] in 2006 determined thio-DMA, thio-DMAA and thio-DMAE in a human urine reference material, NIES 18. The thioarsenicals were reported to represent approximately $10 \%$ of the total urinary arsenic. Interestingly, the human urine used to produce this reference material is not spiked with arsenic species but is collected from Japanese men who were not occupationally exposed to arsenic, but whose diet is rich in seafood, algae and mollusks [30]. Studies have also shown that inorganic arsenic can be converted to thioarsenicals in ground water [48] and algae has also shown to be able to convert inorganic arsenic into both methylated oxo- and thioarsenicals [48], with arsenosugars being the major arsenic compound found in marine algae [50]. This goes some way to explain why thio-DMA is found in human urine from a variety of possible sources of exposure.

The ESI-QqTOF-MS/MS analysis in this study of thio-DMA is in agreement with the LC-ESI-MS/MS analysis of a thio-DMA standard by Fricke [47], who also found product ions at $m / z 137,121$ and 109. However, the ESI-QqTOF-MS/MS analysis in this study also produced product ions corresponding to $\mathrm{m} / \mathrm{z} 127$ and 113. The peak at $\mathrm{m} / \mathrm{z} 127$ is in agreement with Hansen [51] however the actual molecular structure for this signal and $m / z 113$ is unknown.

A study that found an unknown arsenic peak during the speciation of a rice extract (from rice cooked in arsenic contaminated water), also determined their unknown peak to be thio-DMA [52]. They employed the same analytical techniques used in this study to determine its identity; thio-DMA spikes, conversion using $\mathrm{H}_{2} \mathrm{O}_{2}$ and IC-ESI-MS analysis. However, they used negative ion mode MS, meaning peaks were identified at $\mathrm{m} / \mathrm{z}$ 153, 138, 123 and 105.

The urine samples which contained peak 2 came from two sources consisting of two separate hospital patients and UK semi-conductor workers. The only other arsenic species present in both the urine samples of the two separate hospital patients apart from peak 2 was arsenobetaine. This would suggest that the presence of peak 2 was not from inorganic arsenic exposure but most likely from dietary exposure. Furthermore, while the source of exposure for the semi-conductor workers potentially could be occupational inorganic exposure, the speciation analysis of their urine samples indicated otherwise.

Several studies have reported differences in the excretion of oxo- and thioarsenical metabolites after the ingestion of arsenosugars $[28,40,53]$. Suggested possible reasons for this human metabolic variation might be differences in biotransformation enzymes found in the liver, people's differing ability to transform or degrade arsenosugars, differences in gut flora or the ability to uptake arsenosugars [28]. This individual metabolic variation/uniqueness may suggest why these oxo- and thioarsenicals are not seen as frequently as other dietary compounds such as AB during our general arsenic speciation analysis at the Health and Safety Laboratory.

The results outlined in Figure 2F-J and Figure S3 show that when thioarsenicals, thio-DMA and thio-DMAA, are oxidized with $\mathrm{H}_{2} \mathrm{O}_{2}$ they begin to convert to their oxo analogues; $\mathrm{DMA}^{5+}$ and DMAA respectively. However as shown in Figure S2C,F, thio-DMAE and DMAE have identical retention times using the chromatographic conditions used in this study, therefore it is difficult to say if the same oxidation process also applies to thio-DMAE. The fact that peak 1 (thio-DMA) follows 
this same oxidative process and peak 2 does not, may suggest that peak 2 is not a thioarsenical; this hypothesis would agree with the literature where it is stated that the oxidizing agent hydrogen peroxide converts thioarsenicals to their oxoarsenical analogues [27,48]. Therefore, peak 2 could be one of the oxoarsenicals or an arsenosugar not analyzed in this study. The study by Raml [27] reported traces of an arsenosugar in urine but it was not sufficiently separated from the other arsenicals when using an anion exchange column. In addition a study in 2006 by Schmiesser [42] found arsenolipid metabolites in human urine after the ingestion of cod liver, known as arsenic fatty acids such as dimethylarsenopropanoic acid (DMAP), thio-dimethylarsenopropanoic acid (thio-DMAP), dimethylarsenobutanoic acid (DMAB) and thio-dimethylarsenobutanoic acid (thio-DMAB), with all but DMAP were determined using anion exchange chromatography.

Several research groups have recently attempted to better explain arsenic metabolic pathways using the liver of rats [54] and rat or mouse cecum [48,55]. Wang [48] suggests that pre-absorptive methylation of thioarsenicals is facilitated by gastrointestinal microbiota before transport across the gastrointestinal barrier, and post-absorptive formation of thio-arsenicals occurs when oxoarsenicals are converted due to the presence of $\mathrm{H}_{2} \mathrm{~S}$ formed in tissues. They also postulated that during in-vitro reactions both thio-DMA and dithio-DMA occur from $\mathrm{DMA}^{3+}$, while in-vitro human red blood cells take up $\mathrm{DMA}^{3+}$ and quickly convert it to thio-DMA. This research does advance the understanding of these pathways but it is worth noting that Wang [48], Suzuki [54] and Kubachka [55] have all proposed different possible pathways for the in vitro methylation, reduction and thiolation of inorganic arsenic as well as to mono-, di- and trimethylated species with a range of oxo- and thioarsenical products.

\section{Conclusions}

In conclusion, the investigation, by both $\mu \mathrm{LC}-\mathrm{ICP}-\mathrm{MS}$ and ESI-QqTOF-MS/MS, has determined that peak 1 is thio-DMA. Peak 2 has not been identified to date. However, this study has established that peak 2 is not thio-DMA, DMAA, thio-DMAA, DMAE, thio-DMAE, AC or TMAO. The fact that peak 2 occurs with $A B$ in the samples could suggest that its presence is a result of the consumption of seafood. In addition this study has shown that these two compounds occur in urine samples collected from normal and abnormal sources, they have not been observed in the same urine sample and that there may be a suggestion that thio-DMA is a breakdown product of dithio-DMA. The identification of these new arsenicals is extremely challenging for a combination of factors; there is a lack of available compounds; the sensitivity of organic mass spectrometric techniques used for the molecular identification of arsenic compounds and the overall knowledge of arsenic processes. Nevertheless, it is hoped that this study will inform and contribute to the continued research in oxo- and thioarsenicals in human urine samples.

Supplementary Materials: The following are available online at www.mdpi.com/2305-6304/5/2/12/s1, Figure S1: Structures of inorganic arsenic, methylated metabolites, oxo- and thio- dimethylated analogues and dietary arsenic compounds. The structures in blue indicate the seven arsenicals investigated in this study, Figure S2: Chromatograms of seven additional arsenic species and their retention times analysed using an ESI OneFAST system coupled to a Dionex AG7 anion exchange column and ICP-MS using mobile phases $2 \mathrm{mM}$ and $70 \mathrm{mM}$ ammonium carbonate solution (A) the standard five arsenic species (B) DMAA, (C) DMAE, (D) thio-DMA, (E) thio-DMAA (F) thio-DMAE (G) AC and (H) TMAO, Figure S3: Chromatograms of arsenic species analysed using an ESI OneFAST system coupled to a Dionex AG7 anion exchange column and ICP-MS using mobile phases $2 \mathrm{mM}$ and $70 \mathrm{mM}$ ammonium carbonate solution. (A) A urine sample containing peak 2. (B) The urine sample with $100 \% v / v \mathrm{H}_{2} \mathrm{O}_{2}$ added. (C) A thio-DMAA standard spiked into 'blank urine' (only containing AB arsenic species). (D) The spiked urine sample from chromatogram $\mathrm{C}$ with $50 \% v / v \mathrm{H}_{2} \mathrm{O}_{2}$ added. (E) DMAA standard spiked into 'blank urine' (only containing AB arsenic species).

Acknowledgments: The authors would like to thank Kevin Francesconi for providing the oxo- and thioarsenicals: thio-DMA, thio-DMAA, thio-DMAE, DMAA and DMAE used in this study, and Kate Jones and Craig Sams at the Health and Safety Laboratory for their help using tandem mass spectrometry. Funding: This publication and the work it describes were funded by the Health \& Safety Executive (HSE). Its contents, including any opinions and/or conclusions expressed, are those of the authors alone and do not necessarily reflect HSE policy.

Author Contributions: Elizabeth Leese as principal researcher conducted the ICP-MS analysis and wrote the paper. Malcolm Clench conducted the ESI-QqTOF-MS/MS analysis. Elizabeth Leese, Malcolm Clench, Jackie Morton, 
and Vikki Carolan conceived and designed the experiments. Malcolm Clench, Jackie Morton, Philip Gardiner and Vikki Carolan edited the paper.

Conflicts of Interest: The authors declare no conflict of interest.

\section{References}

1. Rehman, K.; Naranmandura, H. Arsenic metabolism and thioarsenicals. Metallomics 2012, 4, 881-892. [CrossRef] [PubMed]

2. Hayakawa, T.; Kobayashi, Y.; Cui, X.; Hirano, S. A new metabolic pathway of arsenite: Arsenic-glutathione complexes are substrates for human arsenic methyltransferase Cyt19. Arch. Toxicol. 2005, 79, 183-191. [CrossRef] [PubMed]

3. Naranmandura, H.; Suzuki, N.; Suzuki, K.T. Trivalent arsenicals are bound to proteins during reductive methylation. Chem. Res. Toxicol. 2006, 19, 1010-1018. [CrossRef] [PubMed]

4. Wang, S.; Li, X.; Song, X.; Geng, Z.; Hu, X.; Wang, Z. Rapid equilibrium kinetiv analysis of arsenite methylation catalysed by recombinant human arsenic (+3 oxidation state) methyltransferase (hAS3MT). J. Biol. Chem. 2012, 287, 38790-38799. [CrossRef] [PubMed]

5. Challenger, F. Biological methylation. Chem. Rev. 1945, 36, 315-361. [CrossRef]

6. Drobna, Z.; Naranmandura, H.; Kubachka, K.M.; Edwards, B.C.; Herbin-Davis, K.; Styblo, M.; Le, X.C.; Creed, J.T.; Maeda, N.; Hughes, M.F.; et al. Disruption of the arsenic (+3 oxidation state) methyltransferase gene in the mouse alters the phenotype for methylation of arsenic and affects distribution and retention of orally administered arsenate. Chem. Res. Toxicol. 2009, 22, 1713-1720. [CrossRef] [PubMed]

7. Hughes, M.F.; Edwards, B.C.; Herbin-Davis, K.M.; Saunders, J.; Styblo, M.; Thomas, D.J. Arsenic (+3 oxidation state) methyltransferase genotype affects steady-state distribution and clearance of arsenic in arsenic-treated mice. Toxicol. Appl. Pharmacol. 2010, 249, 217-223. [CrossRef] [PubMed]

8. Dopp, E.; Kligerman, A.D.; Diaz-Bone, R.A. Organoarsenicals. Uptake, metabolism and toxicity. Met. Ions Life Sci. 2010, 7, 231-265. [PubMed]

9. Roy, P.; Saha, A. Metabolsim and toxicity of arsenic: A human carcinogen. Curr. Sci. 2002, 82, 38-45.

10. Cohen, S.M.; Arnold, L.L. Methylated arsenicals: The implications of metabolism and carcinogenicity studies in rodents to human risk assessment. Crit. Rev. Toxicol. 2006, 36, 99-133. [CrossRef] [PubMed]

11. Drobna, Z.; Walton, F.S.; Paul, D.S.; Xing, W.; Thomas, D.J.; Stýblo, M. Metabolism of arsenic in human liver: The role of membrane transporters. Arch. Toxicol. 2010, 84, 3-16. [CrossRef] [PubMed]

12. Liu, J.; Waalkes, M.P. Liver is a target of arsenic carcinogenesis. Toxicol. Sci. 2008, 105, 24-32. [CrossRef] [PubMed]

13. Sun, G.X.; Williams, P.N.; Zhu, Y.G.; Deacon, C.; Carey, A.M.; Raab, A.; Feldmann, J.; Meharg, A.A. Survey of arsenic and its speciation in rice products such as breakfast cereals, rice crackers and Japanese rice condiments. Environ. Int. 2009, 35, 473-475. [CrossRef] [PubMed]

14. Zhu, Y.G.; Williams, P.N.; Meharg, A.A. Exposure to inorganic arsenic from rice: A global health issue? Environ. Pollut. 2008, 154, 169-171. [CrossRef] [PubMed]

15. Francesconi, K.A.; Tanggaar, R.; McKenzie, C.J.; Goessler, W. Arsenic metabolites in human urine after ingestion of an arsenosugar. Clin. Chem. 2002, 48, 92-101. [PubMed]

16. Niegel, C.; Matysik, F.M. Analytical methods for the determination of arsenosugars-A review of recent trends and developments. Anal. Chim. Acta 2010, 11, 83-99. [CrossRef] [PubMed]

17. Heinrich-Ramm, R.; Mindt-Prufert, S.; Szadkowski, D. Arsenic species excretion after controlled seafood consumption. J. Chromatogr. B 2002, 778, 263-273. [CrossRef]

18. Francesconi, K.A. Arsenic species in seafood: Origin and human health implications. Pure Appl. Chem. 2010, 82, 373-381. [CrossRef]

19. Feldmann, J.; Krupp, E.M. Critical review or scientific opinion paper: Arsenosugars-A class of benign arsenic species or justification for developing partly speciated arsenic fractionation in foodstuffs? Anal. Bioanal. Chem. 2011, 399, 1735-1741. [CrossRef] [PubMed]

20. Thomas, D.J. The chemistry and metabolism of arsenic. In Arsenic: Exposure Sources, Health Risks, and Mechanisms of Toxicity; John Wiley and Sons: Hoboken, NJ, USA, 2015; pp. 81-109. 
21. Pinyayev, T.S.; Kohan, M.J.; Herbin-Davis, K.; Creed, J.T.; Thomas, D.J. Preabsorptive metabolism of sodium arsenate by anaerobic microbiota of mouse cecum forms a variety of methylated and thiolated arsenicals. Chem. Res. Toxicol. 2011, 24, 475-477. [CrossRef] [PubMed]

22. Naranmandura, H.; Suzuki, N.; Suzuki, K.T. Reaction mechanism underlying the in vitro transformation of thioarsenicals. Toxicol. Appl. Pharmacol. 2008, 231, 328-335. [CrossRef] [PubMed]

23. Naranmandura, H.; Carew, M.W.; Xu, S.; Lee, J.; Leslie, E.M.; Weinfeld, M.; Le, X.C. Comparative toxicity of arsenic metabolites in human bladder cancer EJ-1 cells. Chem. Res. Toxicol. 2011, 24, 1586-1596. [CrossRef] [PubMed]

24. Naranmandura, H.; Suzuki, N.; Iwata, K.; Hirano, S.; Suzuki, K.T. Arsenic metabolism and thioarsenicals in hamsters and rats. Chem. Res. Toxicol. 2007, 20, 616-624. [CrossRef] [PubMed]

25. Naranmandura, H.; Iwata, K.; Suzuki, K.T.; Ogra, Y. Distribution and metabolism of four different dimethylated arsenicals in hamsters. Toxicol. Appl. Pharmacol. 2010, 15, 67-75. [CrossRef] [PubMed]

26. Hansen, H.R.; Pickford, R.; Thomas-Oates, J.; Jaspars, M.; Feldmann, J. 2-Dimethylarsinothioyl acetic acid identified in a biological sample: The first occurrence of a mammalian arsinothioyl metabolite. Angew. Chem. Int. Ed. 2004, 43, 337-340. [CrossRef] [PubMed]

27. Raml, R.; Goessler, W.; Traar, P.; Ochi, T.; Francesconi, A.K. Novel thioarsenic metabolites in human urine after ingestion of an arsenosugar, 2',3'-dihydroxypropyl 5-deoxy-5-dimethylarsinoyl-beta-D-riboside. Chem. Res. Toxicol. 2005, 18, 1444-1450. [CrossRef] [PubMed]

28. Raml, R.; Raber, G.; Rumpler, A.; Bauernhofer, T.; Goessler, W.; Francesconi, K.A. Individual variability in the human metabolism of an arsenic containing carbohydrate, $2^{\prime}, 3^{\prime}$-dihydroxypropyl 5-deoxy-5-dimethylarsinoyl-beta-D-riboside, a naturally occurring arsenical in seafood. Chem. Res. Toxicol. 2009, 22, 1534-1540. [CrossRef] [PubMed]

29. Mandal, B.K.; Suzuki, K.T.; Anzai, K.; Yamaguchi, K.; Sei, Y. A SEC-HPLC-ICP MS hyphenated technique for identification of sulphur containing arsenic metabolites in biological samples. J. Chromatogr. B: Anal. Technol. Biomed. Life Sci. 2008, 874, 64-76. [CrossRef] [PubMed]

30. Raml, R.; Goessler, W.; Francesconi, K.A. Improved chromatographic separation of thio-arsenic compounds by reversed-phase high performance liquid chromatography-inductively coupled plasma mass spectrometry. J. Chromatogr. A 2006, 1128, 164-170. [CrossRef] [PubMed]

31. Raml, R.; Rumpler, A.; Goessler, W.; Vahter, M.; Li, L.; Ochi, T.; Francesconi, K.A. Thio-dimethylarsinate is a common metabolite in urine samples from arsenic-exposed women in Bangladesh. Toxicol. Appl. Pharmacol. 2007, 222, 374-380. [CrossRef] [PubMed]

32. Leffers, L.; Ebert, F.; Taleshi, M.S.; Francesconi, K.A.; Schwerdtle, T. In vitro toxicological characterization of two arsenosugars and their metabolites. Mol. Nutr. Food Res. 2013, 57, 1270-1282. [CrossRef] [PubMed]

33. Yoshida, K.; Kuroda, K.; Zhou, X.; Inoue, Y.; Date, Y.; Wanibuchi, H.; Fukushima, S.; Endo, G. Urinary sulphur containing metabolite produced by intestinal bacteria following oral administration of dimethylarsinic acid to rats. Chem. Res. Toxicol. 2003, 16, 1124-1129. [CrossRef] [PubMed]

34. Kuroda, K.; Yoshida, K.; Yoshimura, M.; Endo, Y.; Wanibuchi, H.; Fukushima, S.; Endo, G. Microbial metabolite of dimethylarsinic acid is highly toxic and genotoxic. Toxicol. Appl. Pharmacol. 2004, 198, 345-353. [CrossRef] [PubMed]

35. Naranmandura, H.; Ogra, Y.; Iwata, K.; Lee, J.; Suzuki, K.T.; Weinfeld, M.; Le, X.C. Evidence for toxicity differences between inorganic arsenite and thioarsenicals in human bladder cancer cells. Toxicol. Appl. Pharmacol. 2009, 238, 133-140. [CrossRef] [PubMed]

36. Ebert, F.; Leffers, L.; Weber, T.; Berndt, S.; Mangerich, A.; Beneke, S.; Burkle, A.; Schwerdtle, T. Toxicological properties of the thiolated inorganic arsenic and arsenosugar metabolite thio-dimethylarsinic acid in human bladder cells. J. Trace Elem. Med. Biol. 2014, 28, 138-146. [CrossRef] [PubMed]

37. Leffers, L.; Unterberg, M.; Bartel, M.; Hoppe, C.; Pieper, I.; Stertmann, J.; Ebert, F.; Humpf, H.U.; Schwerdtle, T. In vitro toxicological characterisation of the S-containing arsenic metabolites thio-dimethylarsinic acid and dimethylarsinic glutathione. Toxicology 2013, 305, 109-119. [CrossRef] [PubMed]

38. Bartel, M.; Ebert, F.; Leffers, L.; Karst, U.; Schwerdtle, T. Toxicological characterization of the inorganic and organic arsenic metabolite Thio-DMA in cultured human lung cells. J. Toxicol. 2011, 2011, 373141.

39. Unterberg, M.; Leffers, L.; Hubner, F.; Humpf, H.U.; Lepikhov, K.; Walter, J.; Ebert, F.; Schwerdtle, T. Toxicity of arsenite and thio-DMA(V) after long-term (21 days) incubation of human urothelial cells: Cytotoxicity, genotoxicity and epigenetics. Toxicol. Res. 2014, 3, 456-464. [CrossRef] 
40. Ma, M.S.; Le, X.C. Effect of arsenosugar ingestion on urinary arsenic speciation. Clin. Chem. 1998, 44, 539-550. [PubMed]

41. Molin, M.; Ydersbond, T.A.; Ulven, S.M.; Holck, M.; Dahl, L.; Sloth, J.J.; Fliegel, D.; Goessler, W.; Alexander, J.; Meltzer, H.M. Major and minor arsenic compounds accouting for the total urinary excretion of arsenic following intake of blue mussels (Mytilus edulis): A controlled human study. Food Chem. Toxicol. 2012, 50, 2462-2472. [CrossRef] [PubMed]

42. Schmeisser, E.; Goessler, W.; Francesconi, A.K. Human metabolism of arsenolipids present in cod liver. Anal. Bioanal. Chem. 2006, 385, 367-376. [CrossRef] [PubMed]

43. HSE. Biological Monitoring in the Workplace: A Guide to its Practical Application to Chemical Exposure; HSG 167; HSE Books, 1997.

44. Leese, E.; Morton, J.; Tan, E.; Gardiner, P.H.; Carolan, V.A. $\mu$ LC-ICP-MS determinations of unexposed UK urinary arsenic speciation reference values. J. Anal. Toxicol. 2014, 38, 24-30. [CrossRef] [PubMed]

45. American Conference of Government Industrial Hygienists. TLVs and BEIs: Threshold Limit Values for Chemical Substances and Physical Agents \& Biological Exposure Indices; ACGIH Signature Publications: USA, 2016.

46. Suzuki, K.T.; Katagiri, A.; Sakuma, Y.; Ogra, Y.; Ohmichi, M. Distributions and chemical forms of arsenic after intravenous administration of dimethylarsinic and monomethylarsonic acid to rats. Toxicol. Appl. Pharmacol. 2014, 198, 336-344. [CrossRef] [PubMed]

47. Fricke, M.; Zeller, M.; Cullen, W.; Witkowski, M.; Creed, J. Dimethylthioarsinic anhydride: A standard for arsenic speciation. Anal. Chim. Acta 2007, 583, 78-83. [CrossRef] [PubMed]

48. Wang, Q.Q.; Thomas, D.J.; Naranmandura, H. Importance of being thiomethylated: Formation, fate, and effects of methylated thioarsenicals. Chem. Res. Toxicol. 2015, 28, 281-289. [CrossRef] [PubMed]

49. Conklin, S.D.; Fricke, M.W.; Creed, P.A.; Creed, J.T. Investigation of the pH effects on the formation of methylated thio-arsenicals, and the effects of $\mathrm{pH}$ and temperature on their stability. J. Anal. At. Spectrom. 2008, 23, 711-716. [CrossRef]

50. Francesconi, K.A.; Edmonds, J.S. Arsenic and marine organisms. Adv. Inorg. Chem. 1997, 44, 147-189.

51. Hansen, H.R.; Raab, A.; Jaspars, M.; Milne, B.F.; Feldmann, J. Sulfur containing arsenical mistaken for dimethylarsinous acid [DMA(III)] and identified as a natural metabolite in urine: Major implications for studies on arsenic metabolism and toxicity. Chem. Res. Toxicol. 2004, 17, 1086-1091. [CrossRef] [PubMed]

52. Ackerman, A.H.; Creed, P.A.; Parks, A.N.; Fricke, M.W.; Schwegel, C.A.; Creed, J.T.; Heitkemper, D.T.; Vela, N.P. Comparison of a chemical and enzymatic extraction of arsenic from rice and an assessment of the arsenic absorption from contaminated water by cooked rice. Environ. Sci. Technol. 2005, 39, 5241-5246. [CrossRef] [PubMed]

53. Le, X.C.; Cullen, W.R.; Reimer, K.J. Human urinary arsenic excretion after one-time ingestion of seaweed, crab, and shrimp. Clin. Chem. 1994, 40, 617-624. [PubMed]

54. Suzuki, K.T.; Mandal, B.K.; Katagiri, A.; Sakuma, Y.; Kawakami, A.; Ogra, Y.; Yamaguchi, K.; Sei, Y.; Yamanaka, K.; Anzai, K.; et al. Dimethylthioarsenicals as arsenic metabolites and their chemical preparations. Chem. Res. Toxicol. 2004, 17, 914-921. [CrossRef] [PubMed]

55. Kubachka, K.M.; Kohan, M.C.; Herbin-Davis, K.; Creed, J.T.; Thomas, D.J. Exploring the in vitro formation of trimethylarsine sulfide from dimethylthioarsinic acid in anaerobic microflora of mouse cecum using HPLC-ICP-MS and HPLC-ESI-MS. Toxicol. Appl. Pharmacol. 2009, 239, 137-143. [CrossRef] [PubMed]

(C) 2017 by the authors. Licensee MDPI, Basel, Switzerland. This article is an open access article distributed under the terms and conditions of the Creative Commons Attribution (CC BY) license (http://creativecommons.org/licenses/by/4.0/). 


\section{Supplementary Materials: The Investigation of Unexpected Arsenic Compounds Observed in Routine Biological Monitoring Urinary Speciation Analysis}

Elizabeth Leese, Malcolm Clench, Jackie Morton, Philip HE Gardiner and Vikki A Carolan

\begin{tabular}{|c|c|}
\hline $\begin{array}{c}\text { Inorganic } \\
\text { Arsenic }\end{array}$ & $\begin{array}{cc}\mathrm{O} & \\
\| & \mathrm{HO}-\mathrm{As}-\mathrm{OH} \\
\mathrm{HO}-\mathrm{As}_{\mathrm{S}} \mathrm{OH} & \mathrm{OH} \\
\mathrm{OH} & \mathrm{As}^{3+} \\
\mathrm{As}^{5+} & \text { Arsenite } \\
\text { Arsenate } & \\
\end{array}$ \\
\hline $\begin{array}{l}\text { Methylated } \\
\text { Metabolites }\end{array}$ & $\begin{array}{c}\mathrm{H}_{3} \mathrm{C}-\mathrm{As}-\mathrm{OH} \\
1 \\
\mathrm{OH} \\
\mathrm{MMA}^{3+} \\
\text { Monomethylarsonous acid } \\
\\
\mathrm{H}_{3} \mathrm{C}-\mathrm{As}_{1}-\mathrm{CH}_{3} \\
\mathrm{OH} \\
\mathrm{DMA}^{3+} \\
\text { Dimethylarsinous acid }\end{array}$ \\
\hline $\begin{array}{c}\text { Oxo- } \\
\text { dimethylated } \\
\text { Analogues }\end{array}$ & $\begin{array}{c}\mathrm{H}_{3} \mathrm{C}-\mathrm{AS} \\
\mathrm{H}_{3} \mathrm{C} \\
\text { DMAE } \\
\text { Dimethylarsinoylethanol }\end{array}$ \\
\hline $\begin{array}{c}\text { Thio- } \\
\text { dimethylated } \\
\text { analogues }\end{array}$ & 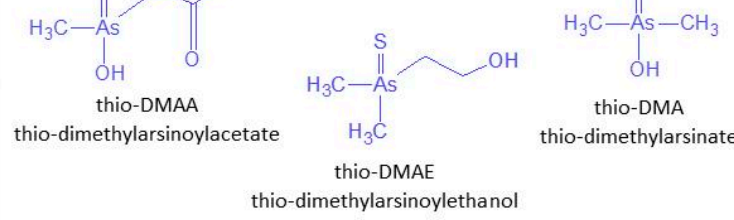 \\
\hline $\begin{array}{l}\text { Dietary } \\
\text { Arsenic }\end{array}$ & $\begin{array}{cc}\mathrm{H}_{3} \mathrm{C}-\mathrm{As}-\mathrm{CH}_{3} & \mathrm{H}_{3} \mathrm{C} \\
\mathrm{CH}_{3} & \mathrm{AC} \\
\text { TMAO } & \text { Arsenocholine } \\
\text { Trimethylarsine Oxide } & \end{array}$ \\
\hline
\end{tabular}

Figure S1. Structures of inorganic arsenic, methylated metabolites, oxo- and thio- dimethylated analogues and dietary arsenic compounds. The structures in blue indicate the seven arsenicals investigated in this study. 

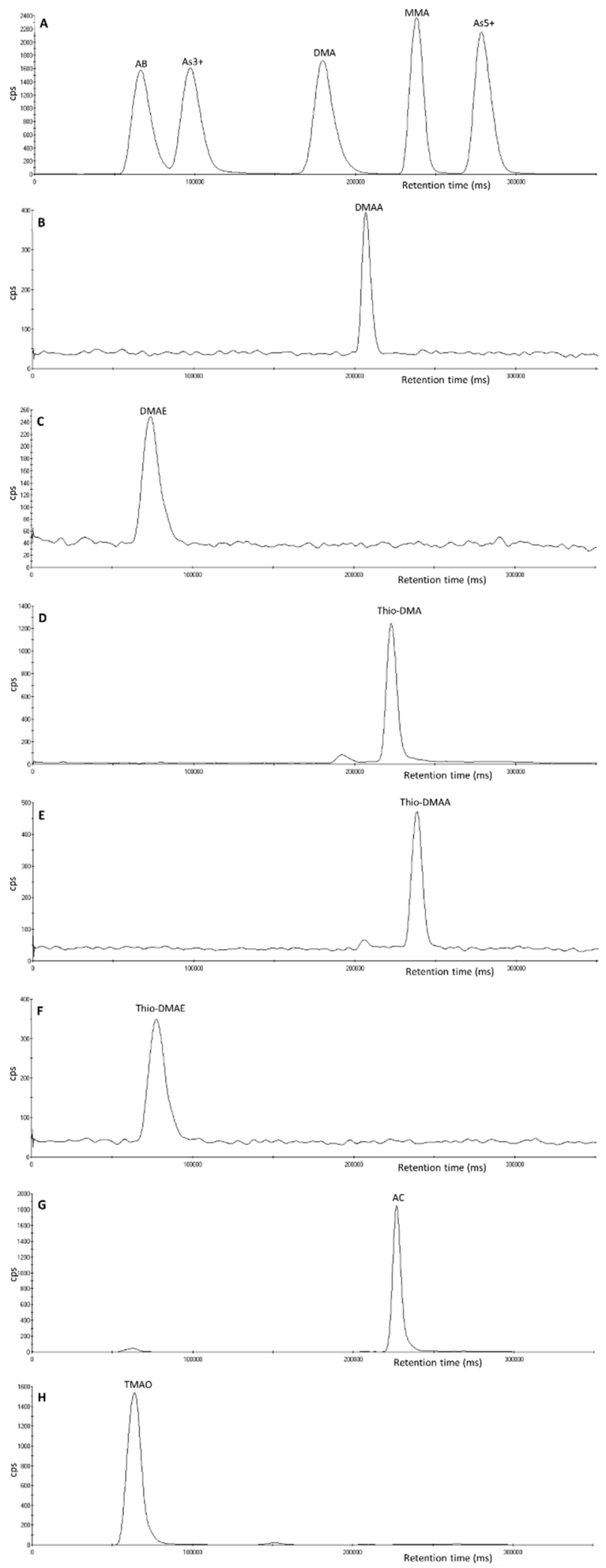

Figure S2. Chromatograms of seven additional arsenic species and their retention times analysed using an ESI OneFAST system coupled to a Dionex AG7 anion exchange column and ICP-MS using mobile phases $2 \mathrm{mM}$ and $70 \mathrm{mM}$ ammonium carbonate solution (A) the standard five arsenic species (B) DMAA, (C) DMAE, (D) thio-DMA, (E) thio-DMAA (F) thio-DMAE (G) AC and (H) TMAO. 

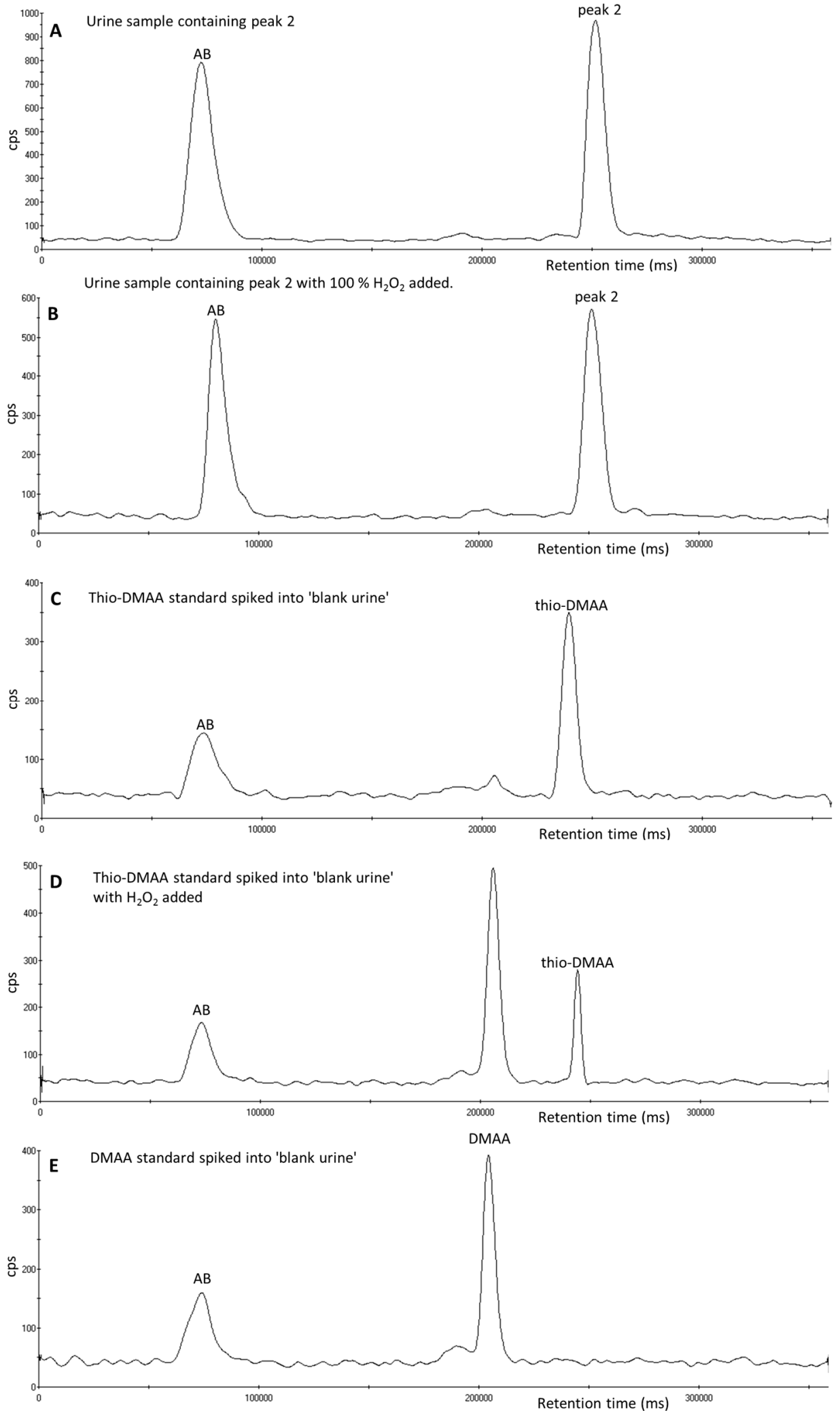

Figure S3. Chromatograms of arsenic species analysed using an ESI OneFAST system coupled to a Dionex AG7 anion exchange column and ICP-MS using mobile phases $2 \mathrm{mM}$ and $70 \mathrm{mM}$ ammonium carbonate solution. (A) A urine sample containing peak 2. (B) The urine sample with $100 \% v / v \mathrm{H}_{2} \mathrm{O}_{2}$ added. (C) A thio-DMAA standard spiked into 'blank urine' (only containing AB arsenic species). (D) The spiked urine sample from chromatogram $\mathrm{C}$ with $50 \% v / v \mathrm{H}_{2} \mathrm{O}_{2}$ added. (E) DMAA standard spiked into 'blank urine' (only containing $\mathrm{AB}$ arsenic species). 


\section{Additional Information}

The first paragraph of section 3.3 in Chapter 3 of this thesis states "Early investigations using tandem mass spectrometry (not cited in the methodology) showed that when monitoring the protonated molecule $[M+H]+m / z 165$ of arsenocholine $(A C)$, no signal was observed at the retention time of an AC standard, when the urine sample containing approximately $2800 \mu \mathrm{gL}^{-1}$ of peak 1 was injected into the MS/MS". The methodology for this is as follows:

Analysis was performed using a liquid chromatography tandem mass spectrometer (LC-MS/MS) (AB Sciex 3200, Warrington, UK). A $1 \mathrm{mg} / \mathrm{L}$ standard solution of AC was injected into the LC-MS by syringe infusion to identify the parent ion $\mathrm{m} / \mathrm{z} 165$ of AC. Using the automated software of the LC-MS, daughter ions m/z 165 and 105 were identified. A $0.1 \mathrm{mg} / \mathrm{L} \mathrm{AC}$ standard and the urine sample containing peak 1 were injected separately into the LC-MS and analysed using the ion transition $\mathrm{m} / \mathrm{z}$ 165/122 and $\mathrm{m} / \mathrm{z}$ 165/105.

The two chromatograms shown in Figure 15, show that the retention time of the 0.1 $\mathrm{mg} / \mathrm{L} \mathrm{AC}$ standard is 2.14 min whilst the retention time of the unknown in the urine sample is $2.56 \mathrm{~min}$. In addition, the ratio of the ion transition m/z 165 to 122 and 105 for the $0.1 \mathrm{mg} / \mathrm{L}$ AC standard is nearly $1: 1$, whereas for the unknown in the urine sample the second transition is approximately less than $20 \%$. Therefore, AC was not thought to be the identity of the peak 1 . 


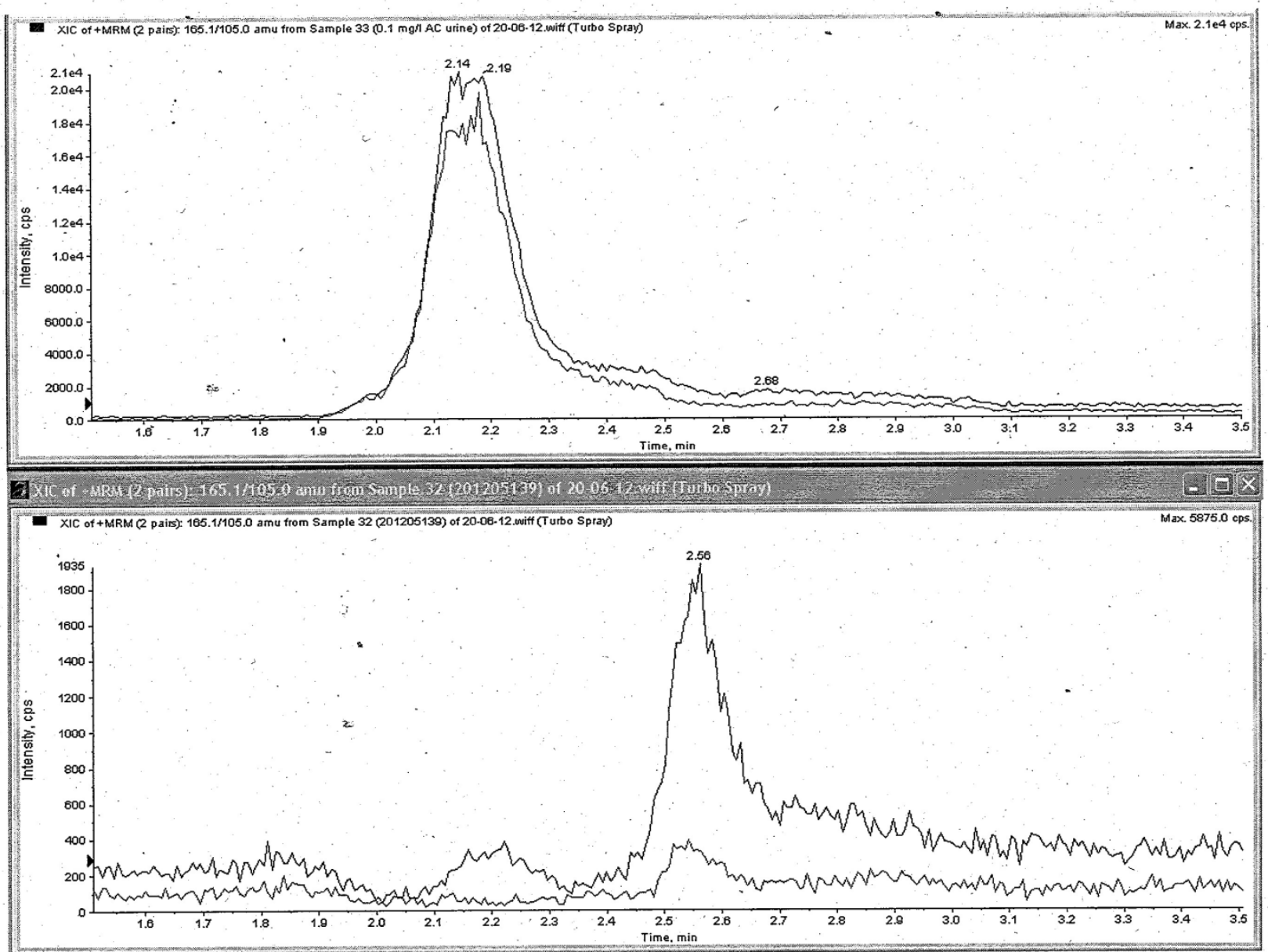

Figure 15 Comparative chromatograms showing the different retention times and ion transition ratios of a $0.1 \mathrm{mg} / \mathrm{L}$ standard of arsenocholine (top) and, a urine sample with unknown peak 1 (bottom). Ion transition ratios monitored were m/z 165/122 and $\mathrm{m} / \mathrm{z}$ 165/105. 


\section{Chapter 4.}

Development of a Method for the Simultaneous Detection of $\mathrm{Cr}(\mathrm{III})$ and Cr(VI) in Exhaled Breath Condensate Samples using $\mu$ LC-ICP-MS.

E. Leese, J. Morton. P.H.E. Gardiner and V.A. Carolan. J. Anal. At. Spectrom., 2016, 31(4): 924-933.

Published - Creative Commons Re-use Open Access Licence

http://pubs.rsc.org/en/content/articlelanding/2016/ja/c5ja00436e\#!divAbstract 


\title{
JAAS
}

\section{PAPER \\ Cite this: J. Anal. At. Spectrom., 2016, detection of $\mathrm{Cr}(\mathrm{II})$ and $\mathrm{Cr}(\mathrm{VI})$ in exhaled breath 31,924 condensate samples using $\mu$ LC-ICP-MS}

\begin{abstract}
Elizabeth Leese, ${ }^{\star a b}$ Jackie Morton, $^{a}$ Philip H. E. Gardiner ${ }^{\mathrm{b}}$ and Vikki A. Carolan ${ }^{\mathrm{b}}$
The method described in this study is a robust and sensitive method for the simultaneous determination of trivalent and hexavalent chromium ( $\mathrm{Cr}(\mathrm{II})$ and $\mathrm{Cr}(\mathrm{VI}))$ in an exhaled breath condensate (EBC) sample and drinking water proficiency testing material sample. The method uses a hyphenated micro liquid chromatography ( $\mu \mathrm{LC}$ ) system coupled to inductively coupled plasma mass spectrometry (ICP-MS). The optimised method incorporates a pH adjusted EDTA complexation step to stabilise $\mathrm{Cr}(\mathrm{VI})$ and $\mathrm{Cr}(\mathrm{II})$. Separation was achieved using an anion exchange micro-sized column. The limits of quantification were $0.040 \mu \mathrm{g} \mathrm{L}^{-1}$ and $0.013 \mu \mathrm{g} \mathrm{L}^{-1}$ for $\mathrm{Cr}(\mathrm{III})$ and $\mathrm{Cr}(\mathrm{VI})$ respectively. EBC is the non-invasive collection of condensate from cooled exhaled breath during regular tidal breathing. We propose that EBC may be a suitable alternative biological matrix to give a more comprehensive picture of exposure to $\mathrm{Cr}(\mathrm{VI})$ following an inhalation exposure. This study shows that water samples and EBC samples containing $\mathrm{Cr}$ (III) and $\mathrm{Cr}(\mathrm{VI})$ can be stored in the fridge $\left(\sim 4^{\circ} \mathrm{C}\right)$ for up to 6 weeks when they have been diluted 10 fold with an EDTA solution, and that EBC samples must not be frozen after collection if the integrity of $\mathrm{Cr}(\mathrm{VI})$ is to be maintained. This study has demonstrated a practical approach to collecting EBC samples from workers potentially occupationally exposed to hexavalent chromium and that the integrity of the chromium species will be maintained prior to analysis.
\end{abstract}

\section{Introduction}

Trivalent chromium is biologically important as an essential element for humans, needed for glucose and lipid metabolism. ${ }^{1}$ Hexavalent chromium however is considered toxic and IARC has classified it as a group 1 agent, defined as carcinogenic to humans. ${ }^{2}$ Hexavalent chromium is known to cause lung and nasal cancer as well as other health conditions such as skin allergies, skin ulcerations, nasal perforations, respiratory problems, kidney and liver damage. ${ }^{3}$ Trivalent chromium $[+3$, $\mathrm{Cr}(\mathrm{III})]$ is the most common form of chromium, whilst hexavalent chromium $[+6, \mathrm{Cr}(\mathrm{vI})]$ is rarely found naturally in the environment, therefore the majority of hexavalent chromium is a product of industry and manufacturing. ${ }^{4}$

Chromium compounds are occupationally important in many industries such as the production and welding of stainless steel including cutting and finishing, electroplating, smelting, pigments and paints used in aerospace and maritime industries, construction and those using pesticides and tanning agents. ${ }^{4}$ Exposure to $\mathrm{Cr}(\mathrm{vI})$ and its compounds can occur by ingestion or dermal exposure but in some industries the

${ }^{a}$ Health and Safety Executive, Harpur Hill, Buxton, Derbyshire, SK17 9JN, UK. E-mail: Liz.leese@hsl.gsi.gov.uk; Tel: +44 (0)1298218434

${ }^{\circ}$ Biomolecular Sciences Research Centre, Sheffield Hallam University, Howard Street, Sheffield $S 11 \mathrm{WB}$, UK inhalation of fumes and mists is most likely. Upon exposure to $\mathrm{Cr}(\mathrm{vI})$, the body naturally reduces $\mathrm{Cr}(\mathrm{VI})$ to $\mathrm{Cr}(\mathrm{III})^{5,6}$ before it is excreted in the urine, with studies suggesting occupational exposure to chromium mainly via inhalation results in elimination half-lives of 7 hours and 15-30 days. ${ }^{7}$ Apart from in bacterial cells, there is no evidence of humans or other biological systems having the ability to re-oxidise $\mathrm{Cr}(\mathrm{III})$ back to $\mathrm{Cr}(\mathrm{vI}) .^{8}$ One approach to assessing occupational exposure uses biological monitoring, involving the measurement of total chromium (without differentiation of $\mathrm{Cr}(\mathrm{III})$ and $\mathrm{Cr}(\mathrm{VI})$ ) in a urine sample. Background unexposed reference ranges and occupational guidance values for total chromium in urine have been established in the UK, ${ }^{9}$ Germany ${ }^{10}$ and the US. ${ }^{11} \mathrm{Cr}(\mathrm{vI})$ has the ability to permeate cell membranes meaning $\mathrm{Cr}(\mathrm{vI})$ can also be measured in red blood cells (RBC), ${ }^{12,13}$ determined as $\mathrm{Cr}(\mathrm{III})$. Once inside $\mathrm{RBCs} \mathrm{Cr}(\mathrm{VI})$ is rapidly reduced to $\mathrm{Cr}(\mathrm{III})$ as it binds with haemoglobin and other proteins. ${ }^{6,12}$ Methods for the separate measurements of $\mathrm{Cr}(\mathrm{III})$ and $\mathrm{Cr}(\mathrm{VI})$ have focused on the assessment of environmental sources of exposure such as natural water, ${ }^{14,15}$ drinking water, ${ }^{14,15}$ waste water ${ }^{16}$ and industrial waste. ${ }^{17}$

Whilst the measurement of total chromium in urine is a practical bio-monitoring approach, it does not give a comprehensive picture of exposure. It is possible that an alternative biological matrix to measure exposure to $\mathrm{Cr}(\mathrm{vI})$ for those exposed through inhalation might be exhaled breath 
condensate (EBC). EBC is the non-invasive collection of condensate from cooled exhaled breath during regular tidal breathing. The collection of exhaled air will contain mostly water vapour but also droplets of fluid from the respiratory tract, ${ }^{18}$ meaning EBC measurements will reflect markers and molecules from the mouth, tracheobronchial system and the alveoli. ${ }^{19} \mathrm{EBC}$ is reported to contain both volatile substances in the gaseous phase along with low volatile and non-volatile substances in droplets. ${ }^{20}$ The non-volatile substances will include salts, proteins, lipids, and environmental contaminants. ${ }^{18-20}$ Because EBC is relatively free of the interfering solutes found in other biological matrices it represents a suitable biological matrix for elemental analysis to assess inhalation exposure to trace elements. Studies have already shown that occupationally inhaled elements have been detected in EBC for cobalt and tungsten, ${ }^{21,22}$ iron, nickel and total chromium, ${ }^{23,24}$ manganese ${ }^{25}$ and hexavalent chromium (using liquid/liquid solvent extraction and analysis by electrothermal atomic absorption spectrometry). ${ }^{26}$ There are currently no publications for the simultaneous detection and determination of $\mathrm{Cr}(\mathrm{III})$ and $\mathrm{Cr}(\mathrm{vI})$ in an EBC sample. Due to the difference in toxicities of $\mathrm{Cr}(\mathrm{III})$ and $\mathrm{Cr}(\mathrm{VI})$ and the conversion from $\mathrm{Cr}(\mathrm{VI})$ to $\mathrm{Cr}(\mathrm{III})$ in the human body after exposure, it is important to measure both species of chromium to enable a more comprehensive picture of exposure assessment.

In order to measure $\mathrm{Cr}(\mathrm{III})$ and $\mathrm{Cr}(\mathrm{VI})$ it is necessary to maintain the integrity of each species in the sample. The stability of both trivalent and hexavalent chromium depends on $\mathrm{pH}$. In acidic solutions, trivalent chromium compounds mainly exist as $\mathrm{Cr}\left(\mathrm{H}_{2} \mathrm{O}\right)_{6}{ }^{3+}$ and hydrolyse to a hydroxide in less acidic and alkaline conditions. Hexavalent chromium compounds exist either as a chromate $\left(\mathrm{CrO}_{4}{ }^{2-}, \mathrm{HCrO}_{4}{ }^{-}, \mathrm{H}_{2} \mathrm{CrO}_{4}\right)$ or dichromate $\left(\mathrm{Cr}_{2} \mathrm{O}_{7}{ }^{2-}\right) \cdot{ }^{3,27}$ In extremely acidic conditions only, hexavalent chromium exists as $\mathrm{H}_{2} \mathrm{CrO}_{4}$; in these acidic conditions hexavalent chromium is a potent oxidant, which after oxidising can also begin to reduce to trivalent chromium compounds. In the $\mathrm{pH}$ range of 2-6 hexavalent chromium compounds exist as $\mathrm{HCrO}_{4}^{-}$and $\mathrm{HCr}_{2} \mathrm{O}_{7}^{-}$, with $\mathrm{HCr}_{2} \mathrm{O}_{7}^{-}$ reducing to $\mathrm{Cr}_{2} \mathrm{O}_{7}{ }^{2-}$ at concentrations higher than $10^{-2} \mathrm{M}$. Above $\mathrm{pH} 6$, hexavalent chromium only exists as $\mathrm{CrO}_{4}{ }^{2-} \cdot{ }^{3,8,27,28}$ Due to this $\mathrm{pH}$ dependence and the fact that trivalent chromium compounds are typically cationic in solution and hexavalent chromium compounds are typically anionic in solution, speciation using ion exchange chromatography methodologies is an analytical challenge. These challenges have resulted in analysis either only determining $\mathrm{Cr}(\mathrm{vI})$ using colorimetric analysis with diphenylcarbohydrazide (DPC) ${ }^{15}$ or by determining both $\mathrm{Cr}(\mathrm{vI})$ by anion chromatography and total chromium to calculate $\mathrm{Cr}(\mathrm{III})$ by subtraction. ${ }^{14} \mathrm{Cr}(\mathrm{III})$ and $\mathrm{Cr}(\mathrm{VI})$ have been simultaneously determined by ICP-MS using reverse phase chromatography combined with ion-pairing reagents. ${ }^{27,29}$ The use of non-traditional HPLC chromatographic methods such as solid phase extraction based fractionation, ${ }^{14,30,31}$ micro columns (PTFE tubes filled with activated alumina) ${ }^{32-35}$ and short column capillary electrophoresis ${ }^{36}$ with subsequent detection by AAS, ICP-AES or ICP-MS have all been employed as simple techniques to separate and determine $\mathrm{Cr}(\mathrm{III})$ and $\mathrm{Cr}(\mathrm{VI})$. Whilst all these methods have their own advantages and disadvantages (such as interferences, contamination, raised backgrounds, unsuitable limits of detection and time consuming procedures), it has meant that other research has focused on anion exchange chromatography coupled to ICP-MS. Both Chen et al. ${ }^{37}$ and Pantsar-Kallio and Manninen ${ }^{38}$ employed a dual column approach to overcome the issue of the opposite charges of the chromium species. Other authors overcame this ionic challenge by using derivatisation, where $\mathrm{Cr}$ (III) is complexed with EDTA to change the ionic charge from a cation to an anion complex, enabling separation of the two chromium species using the same sample injection on an anion exchange column. ${ }^{39,40}$ Suzuki and Serita ${ }^{41}$ were the first to report this technique using HPLC-AAS, with Chen et al. ${ }^{37}$ reporting that alternative complexing agents such as diethylenetriaminepentaacetic acid (DTPA) and 2,6-pyridinedicarboxylic acid (PDCA) were not as effective as EDTA. Other publications have reported the successful speciation of $\mathrm{Cr}(\mathrm{III})$ and $\mathrm{Cr}(\mathrm{VI})$ using newly introduced speciality anion columns with sulfonic and alkyl quaternary ammonium functional groups which boast both anionic and cationic properties, and have been employed by authors concerned with species' integrity related to sample pretreatment. ${ }^{42-44}$

The initial aim of this study was to develop a speciation method to simultaneously determine both $\mathrm{Cr}(\mathrm{III})$ and $\mathrm{Cr}(\mathrm{VI})$ using a small anion exchange speciality column with cationic capabilities using a OneFAST system coupled to an ICP-MS. The method developed was then used to investigate the stability of both chromium species in an EBC sample with the view to establishing a viable technique to employ for workplace monitoring. The objective, being to establish a method to allow the collection and analysis of EBC samples from workers who may be occupationally exposed to hexavalent chromium.

\section{Experimental}

\section{Reagents}

Chromium speciation compounds potassium dichromate $\left(\mathrm{K}_{2} \mathrm{Cr}_{2} \mathrm{O}_{7}\right)$ and chromium(III) chloride $\left(\mathrm{CrCl}_{3}\right)$ were obtained from Fisher Scientific (Loughborough, UK). Ammonia solution $\left(\mathrm{NH}_{3}\right)$, UpA nitric acid and UpA hydrochloric acid was supplied by Romil (UK). Butan-1-ol was from Fisher Scientific (Loughborough, UK).

All solutions were made using ultrapure deionised water $(18.2 \mathrm{M} \Omega \mathrm{hm} \mathrm{cm}$ ) from a Millipore system (Merck Millipore, Billerica, MA, USA). Single standard stock solutions of $1000 \mathrm{mg} \mathrm{L}^{-1}$ of $\mathrm{Cr}(\mathrm{III})$ and $\mathrm{Cr}(\mathrm{VI})$ were prepared fresh in water for each day of analysis.

A commercially available proficiency testing material (PTM) from Sigma Aldrich (UK) for $\mathrm{Cr}(\mathrm{vI})$ in drinking water (Sample WS13-4 Lot LRAA1427) was used to validate the method. Manufacturer instructions suggest the PTM is diluted 100 fold in water. However for this study the PTM was diluted 1000 fold in water to more accurately reflect the levels of $\mathrm{Cr}(\mathrm{vI})$ expected in EBC samples. Previous studies that measured total chromium in $\mathrm{EBC}^{24}$ or $\mathrm{Cr}(\mathrm{vI})$ only in $\mathrm{EBC}^{45}$ reported levels less than $10 \mu \mathrm{g}$ $\mathrm{L}^{-1}$ and $5 \mu \mathrm{g} \mathrm{\textrm {L } ^ { - 1 }}$ of chromium in EBC respectively. 


\section{Instrumentation}

Separation was achieved using a hyphenated $\mu$ LC system with an ICP-MS (XSERIES 2, Thermo Fisher Scientific, Hemel Hempstead, UK). The separation of $\mathrm{Cr}(\mathrm{III})$ and $\mathrm{Cr}(\mathrm{VI})$ was achieved using a $5 \mathrm{~cm}$ anion exchange guard column, (Dionex IONPAC AG7 $4 \mathrm{~mm} \times 50 \mathrm{~mm}$ i.d., $10 \mu \mathrm{m}$, Thermo Fisher Scientific). The Dionex AG7 anion exchange column has cationic exchange capability due to the presence of sulfonic and alkyl quaternary ammonium functional groups, and is suitable for a full $\mathrm{pH}$ range of $0-14$. The micro-flow delivery of both sample and mobile phase was accomplished using an ESI OneFAST system (Elemental Scientific, Warrington, UK), with a six port switching valve and $500 \mu \mathrm{L}$ sample loop. The ICP-MS was operated in collision cell (CCT) mode using $7 \%$ hydrogen in helium (approximately $3.5 \mathrm{~mL} \mathrm{~min}^{-1}$ ). The ICP-MS conditions were optimised daily using a $10 \mu \mathrm{g} \mathrm{L}^{-1}$ tuning solution containing arsenic, chromium, cobalt, indium and uranium (made in $1 \%(\mathrm{v} / \mathrm{v})$ nitric acid from $1000 \mathrm{mg} \mathrm{L}^{-1}$ stock standards (ICPMS standards, BDH, Poole, UK)). Table 1 summarises the ICPMS and OneFAST operating conditions.

\section{Exhaled breath condensate collection}

EBC was collected using a portable TURBO-DECCS condenser (Transportable Unit for Research on Biomarkers Obtained from Disposable Exhaled Condensate Collection Systems) from ItalChill (Parma, Italy). This technique consists of a disposable respiratory system comprising of a mouthpiece connected to a one-way aspiration valve and saliva trap with an EBC sample collection tube at the end inserted into a temperature controlled chilling unit set at $-5{ }^{\circ} \mathrm{C}$ (see Fig. 1). The collection of exhaled

Table 1 Operating conditions of the ICP-MS and OneFAST system

\begin{tabular}{ll}
\hline XSERIES2 ICP-MS Settings & Parameters \\
\hline RF power & $1400 \mathrm{~W}$ \\
Nebuliser gas flow & $0.89-0.90 \mathrm{~L} \mathrm{~min}^{-1}$ \\
Sample/skimmer cones & $\mathrm{Ni} / \mathrm{Ni} \mathrm{Xt}$ cones \\
Collision gas $7 \% \mathrm{He}^{+} \mathrm{H}_{2}$ flow rate & $3.5 \mathrm{~mL} \mathrm{~min}{ }^{-1}$ \\
Isotope monitored & $52 \mathrm{Cr}$ \\
Dwell time & $100 \mathrm{~ms}$ \\
\hline$\mu \mathrm{LC}$ conditions & Parameters \\
\hline & \\
Analytical column & Dionex IONPAC AG7 (4 mm \\
& $\times 50 \mathrm{~mm}, 10 \mu \mathrm{m})$ \\
Mobile phase & $4 \% \mathrm{v} / \mathrm{v}$ ammonia solution \& \\
& $3.2 \% \mathrm{v} / \mathrm{v}$ nitric acid, pH \\
Sample injection time & $1.8-2$ \\
Flow rate & $30 \mathrm{~s}$ \\
Total acquisition time & $1 \mathrm{~mL}$ min \\
Sample loop & $230 \mathrm{~s}$ \\
Peristaltic pump tubing & $500 \mu \mathrm{L}$ \\
ESI switching valve & $\mathrm{Green} /$ orange $0.38 \mathrm{~mm}$ \\
Valve to column connector & P6 - 6 port \\
Column to ICP-MS nebuliser & $0.25 \mathrm{ID}$ (green) \\
Nebuliser & $0.5 \mathrm{ID}($ orange) \\
& PFA-ST
\end{tabular}

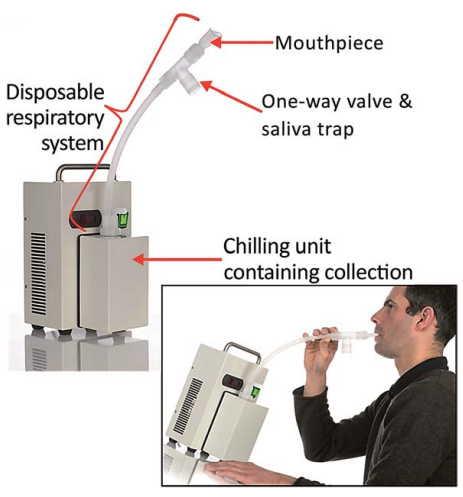

Fig. 1 TURBO-DECCS condenser consisting of a disposable respiratory system comprising of a mouthpiece connected to a one-way aspiration valve and saliva trap with an EBC sample collection tube at the end inserted into a temperature controlled chilling unit set at $-5^{\circ} \mathrm{C}$.

air results in low sample volumes of EBC; 15 minutes of regular tidal breathing will typically provide less than $2 \mathrm{~mL}$ of EBC sample. It was necessary to collect large volumes of EBC from a volunteer not occupationally exposed to hexavalent chromium for use in method development as a blank sample and for recovery. In addition to the volunteer supplying the bulk EBC, five further volunteers supplied EBC samples for the optimised method applications. Blank EBC was collected from the volunteer by regular tidal breathing through the mouth piece of the TURBO-DECCS. For the most part a complete seal around the mouthpiece with the mouth is maintained allowing periodic swallowing of any accumulated saliva.

\section{Method development}

See Fig. 2 for a flow chart outlining the six stages of the method development.

\section{Sample storage experiment}

1. Standards made in water. Individual chromium species standards of $\mathrm{Cr}(\mathrm{III})$ and $\mathrm{Cr}(\mathrm{VI})$, along with a mixed species standard, at a concentration of $5 \mu \mathrm{g} \mathrm{L}^{-1}$ were prepared in water. Four aliquots of each of the three standards were pipetted into individual sample tubes. The first set of aliquots was analysed by $\mu$ LC-ICP-MS immediately after preparation; the remaining sets were capped and one of each standard was stored in the freezer $\left(\sim-20{ }^{\circ} \mathrm{C}\right)$, the fridge $\left(\sim 4{ }^{\circ} \mathrm{C}\right)$ and at laboratory room temperature $\left(\sim 22{ }^{\circ} \mathrm{C}\right)$ for 24 hours. After 24 hours storage each aliquot was removed from their respective storage conditions, placed on a roller mixer and left to reach room temperature. After reaching room temperature each aliquot was analysed by $\mu$ LC-ICP-MS.

2. Derivatisation by complexation of $\mathrm{Cr}$ (III) with EDTA. A solution of $0.5 \mathrm{mM}$ EDTA was made in water and used to prepare a $5 \mu \mathrm{g} \mathrm{L} \mathrm{L}^{-1} \mathrm{Cr}$ (III) standard. The standard was analysed immediately and again in 1 hour after being left to stand at room temperature. 


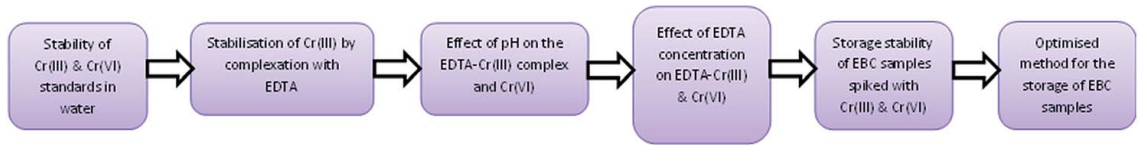

Fig. 2 Flow chart showing optimisation stages of the method development.

3. Effect of pH on the EDTA-Cr(III) complex and $\mathrm{Cr}(\mathrm{vI})$. In a preparation of $0.5 \mathrm{mM}$ EDTA in water, the $\mathrm{pH}$ is 5 . Six solutions of $0.5 \mathrm{mM}$ EDTA were prepared; five of those solutions were adjusted to achieve six different $\mathrm{pH}$ readings; $\mathrm{pH} 4,5,7$, 7.6, 8 and $9.10 \% \mathrm{v} / \mathrm{v}$ nitric acid was used to adjust to $\mathrm{pH} 4$ and $10 \% \mathrm{v} / \mathrm{v}$ ammonia solution was used to adjust to $\mathrm{pH} 7,7.6,8$ and 9.

4. Concentration of EDTA. To investigate the influence of the concentration of the EDTA solution on either retention time of the chromium species or peak shape a $5 \mu \mathrm{g} \mathrm{L} \mathrm{L}^{-1}$ mixed species standard was prepared in $0.5 \mathrm{mM}, 0.6 \mathrm{mM}$ and $0.7 \mathrm{mM}$ EDTA solutions. Each solution was adjusted to $\mathrm{pH} 8$ using $10 \% \mathrm{v} / \mathrm{v}$ ammonia solution, and left to stand at room temperature for 1 hour prior to analysis.

5. Storage stability of EBC samples. Two solutions of $0.5 \mathrm{mM}$ EDTA were prepared and adjusted to $\mathrm{pH} 7.6$ and $\mathrm{pH} 8$ using $10 \% \mathrm{v} / \mathrm{v}$ ammonia solution. Two equal volumes of "blank" EBC were spiked with $5 \mu \mathrm{g} \mathrm{L}^{-1} \mathrm{Cr}(\mathrm{III})$ and $5 \mu \mathrm{g} \mathrm{L}^{-1} \mathrm{Cr}(\mathrm{vI})$. Each of
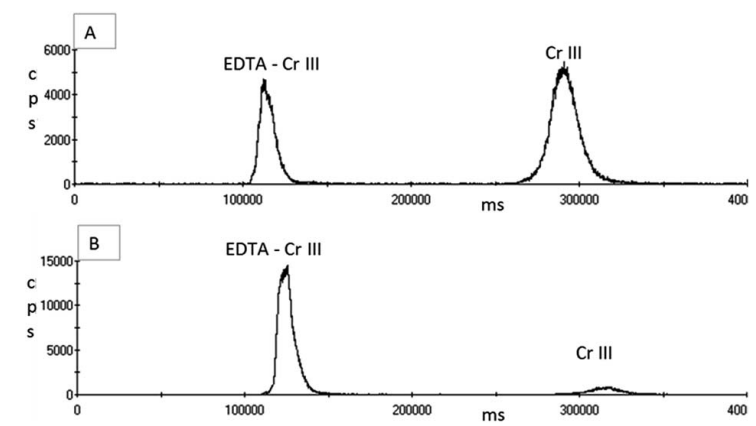

Fig. 4 Chromatograms of a $5 \mu \mathrm{g} \mathrm{L}{ }^{-1} \mathrm{Cr}($ (II) $)$ standard prepared in 0.5 mM EDTA analysed using an ESI OneFAST system coupled to a Dionex AG7 anion exchange column and ICP-MS using a nitric acid and ammonia mixed mobile phase. (A) Analysed immediately after preparation. (B) Analysed after being left to stand for 1 hour.
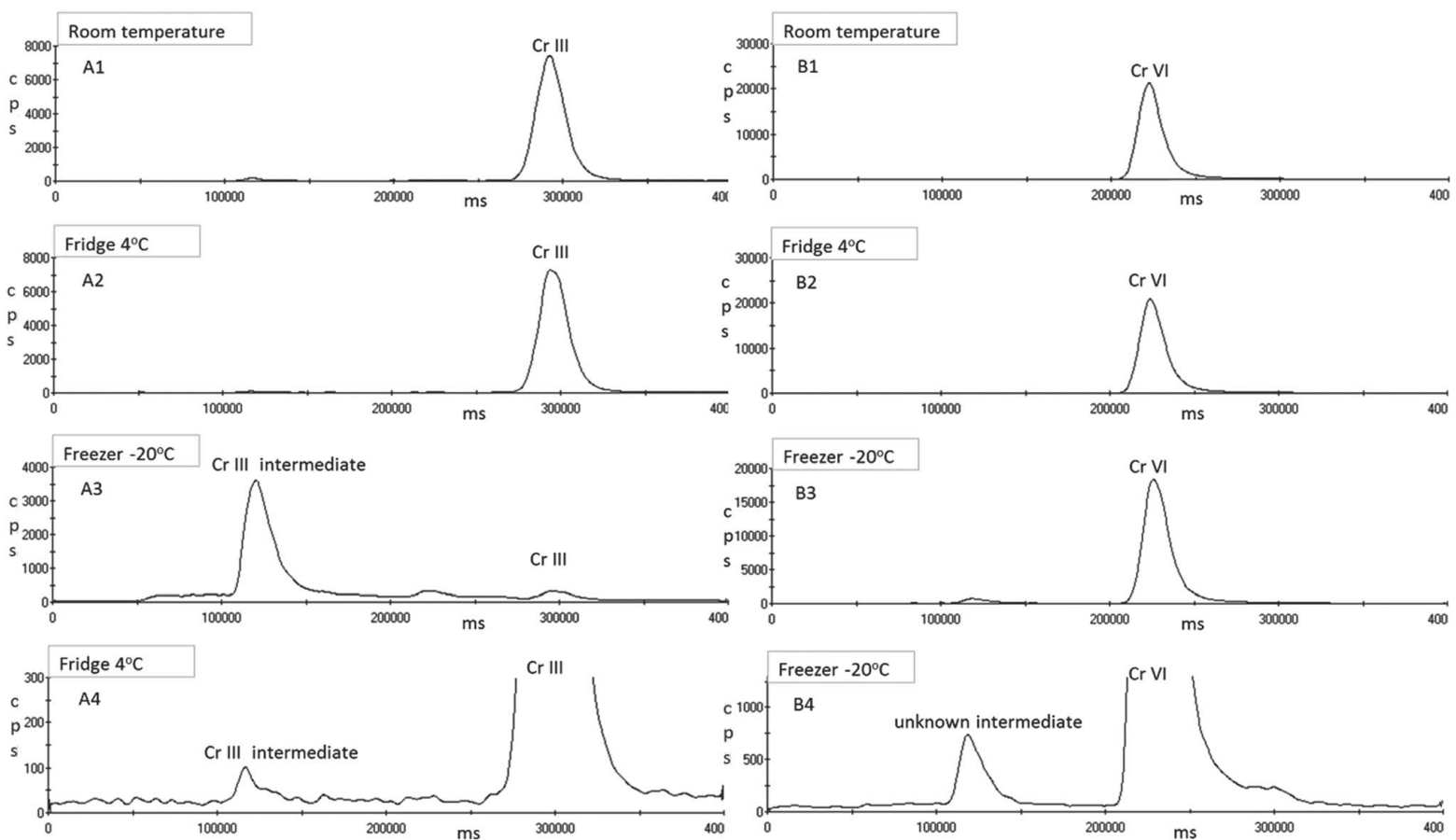

Fig. 3 Chromatograms of $5 \mu \mathrm{g} \mathrm{L}^{-1}$ single standards of $\mathrm{Cr}(\mathrm{VI})$ and $\mathrm{Cr}(\mathrm{III})$ analysed using an ESI OneFAST system coupled to a Dionex AG7 anion exchange column and ICP-MS using a nitric acid and ammonia mixed mobile phase. (A1-A4) Chromatograms of Cr(III). (B1-B4) Chromatograms of $\mathrm{Cr}(\mathrm{vl})$. (A1 \& B1) Chromium species stored at laboratory room temperature ( $22^{\circ} \mathrm{C}$ ). (A2 \& B2) Chromium species stored at fridge temperature $\left(4{ }^{\circ} \mathrm{C}\right)$. (A3 \& B3) Chromium species stored at freezer temperature $\left(-20^{\circ} \mathrm{C}\right)$ for 24 hour. (A4) Baseline of chromatogram (A2) showing $\mathrm{Cr}(\mathrm{\prime \prime})$ intermediate compound. (B4) Baseline of chromatogram (B3) showing the unknown chromium intermediate compound. 

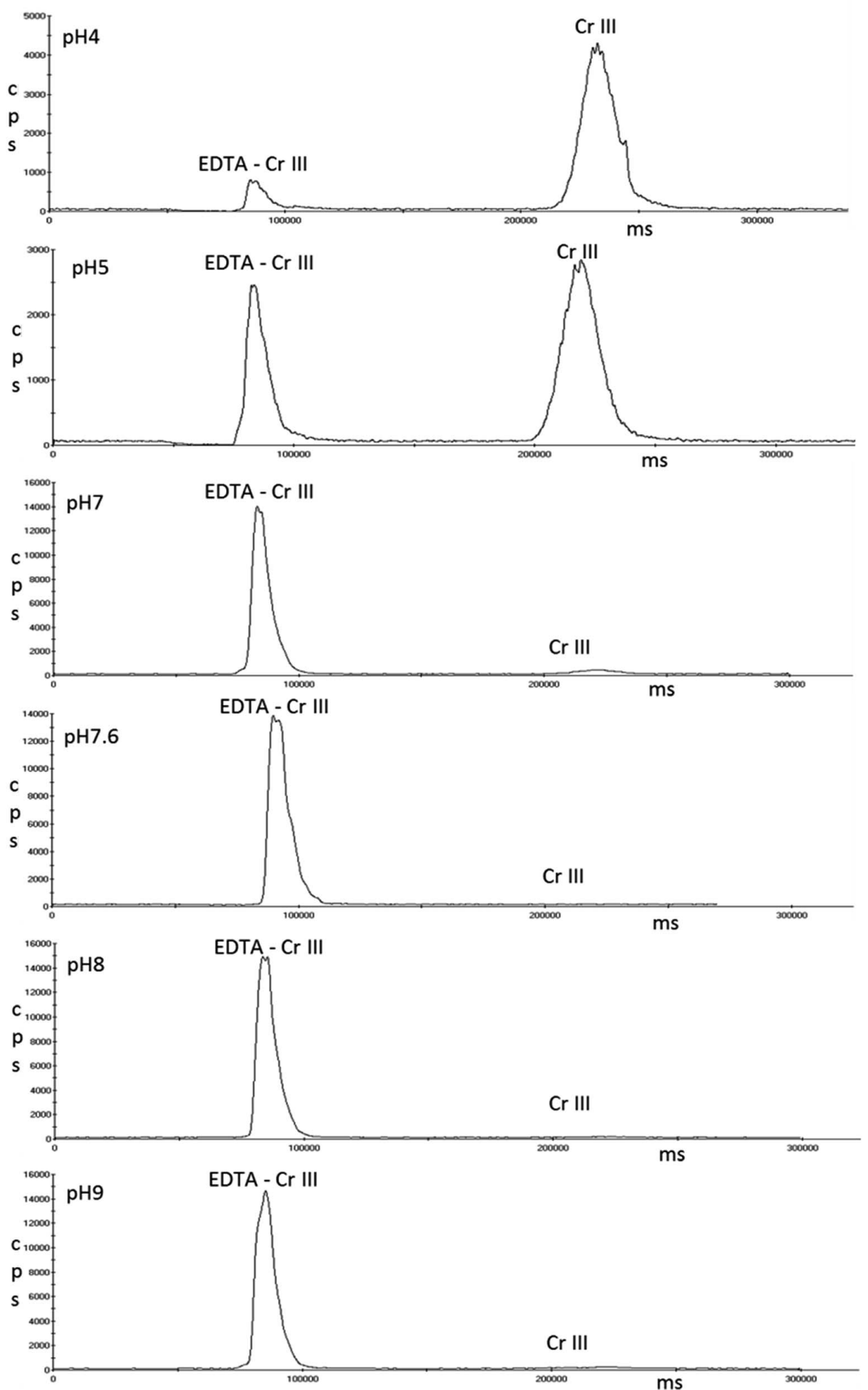

Fig. 5 Chromatograms showing the effect of pH of the EDTA solution to successfully complex and stabilise $\mathrm{Cr}($ (III), analysed using an ESI OneFAST system coupled to a Dionex AG7 anion exchange column and ICP-MS using a nitric acid and ammonia mixed mobile phase. 
the two spiked concentrations of EBC and a "blank" unspiked EBC sample were aliquoted in triplicate for four sets of samples, and then each diluted 10 fold with the $\mathrm{pH} 7.6$ solution of $0.5 \mathrm{mM}$ EDTA. Four further sets of these three samples were prepared and diluted 10 fold with the $\mathrm{pH} 8$ solution of $0.5 \mathrm{mM}$ EDTA. All samples were capped and left to stand at room temperature for 1 hour. One of the four sets of samples of each $\mathrm{pH}$ were analysed by $\mu \mathrm{LC}$-ICP-MS. The remaining sets were stored in the fridge $\left(\sim 4{ }^{\circ} \mathrm{C}\right)$, and analysed at 1 week, 4 weeks and 6 weeks.

6. Optimised method. Separation of $\mathrm{Cr}(\mathrm{III})$ and $\mathrm{Cr}(\mathrm{VI})$ was achieved using a Dionex AG7 column and an isocratic flow of ammonia solution and nitric acid mobile phase. The mobile phase was prepared daily in ultrapure deionised water, by adding $4 \% \mathrm{v} / \mathrm{v}$ ammonia solution and $3.2 \% \mathrm{v} / \mathrm{v}$ ultrapure nitric acid. The $\mathrm{pH}$ was adjusted to between $\mathrm{pH}$ 1.8-2 using the ammonia solution. The flow rate of both the mobile phase and sample injection onto the column is controlled by the ICP-MS peristaltic pump at a constant $1 \mathrm{~mL} \mathrm{~min}^{-1}$. The sample is injected onto the column for 30 seconds and overall sample acquisition time is 230 seconds.

The optimised method incorporates a derivatisation step, with a 10 fold dilution of the EDTA solution (at a concentration of $0.5 \mathrm{mM}$ adjusted to $\mathrm{pH} 8$ with $10 \% \mathrm{v} / \mathrm{v}$ ammonia solution) with the EBC sample. From the $1000 \mathrm{mg} \mathrm{L}^{-1}$ single standard stock solutions of $\mathrm{Cr}(\mathrm{III})$ and $\mathrm{Cr}(\mathrm{VI}) 100 \mu \mathrm{g} \mathrm{\textrm {L } ^ { - 1 }}$ single and mixed species working solutions were prepared daily. Using the $100 \mu \mathrm{g} \mathrm{L}^{-1}$ mixed species working solution, calibration standards of $0.01,0.02,0.05,0.1,0.5,1,2$ and $5 \mu \mathrm{g} \mathrm{L} \mathrm{L}^{-1}$ were prepared in the $0.5 \mathrm{mM}$ EDTA solution using a 10 fold dilution to $50 \mathrm{~mL}$. Blank and spiked EBC samples were diluted 10 fold with the EDTA solution to $1.5 \mathrm{~mL}$. The original 1 in 1000 preparation of the proficiency testing material to validate the method was subsequently diluted 10 fold to $50 \mathrm{~mL}$ with the EDTA solution. All samples including standards were left to stand at room temperature for a minimum of 1 hour prior to analysis by $\mu$ LC-ICP-MS

\section{Results \& discussion}

\section{Method development}

Following initial work with the AG7 ion exchange column, the simultaneous separation of $\mathrm{Cr}(\mathrm{III})$ and $\mathrm{Cr}(\mathrm{VI})$ was achieved in spiked aqueous samples using an isocratic flow of $4 \% \mathrm{v} / \mathrm{v}$ ammonia solution and $3.2 \% \mathrm{v} / \mathrm{v}$ ultrapure nitric acid, with $\mathrm{pH}$ adjustment to between $\mathrm{pH} 1.8-2$ using the ammonia solution.

1. Standards made in water. A spiked aqueous standard was stored at various temperatures for 24 hours prior to analysis
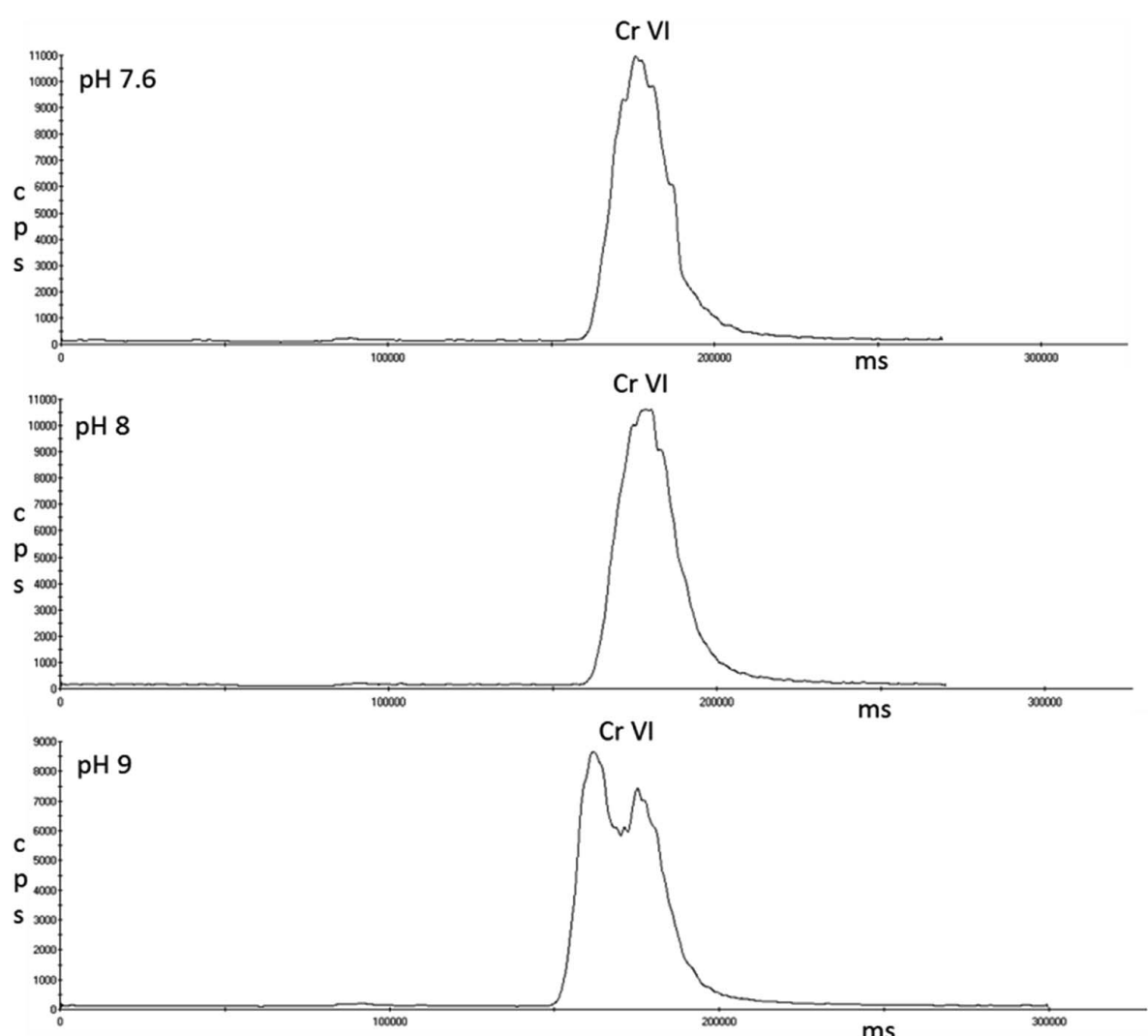

Fig. 6 Chromatograms showing the effect of $\mathrm{pH}$ of the EDTA solution on $\mathrm{Cr}(\mathrm{VI})$ analysed using an ESI OneFAST system coupled to a Dionex AG7 anion exchange column and ICP-MS using a nitric acid and ammonia mixed mobile phase. 
to determine stability. The results (see Fig. 3) show that $\mathrm{Cr}(\mathrm{vI})$ was stable when stored at both room temperature and refrigerated, but species instability and conversion of $\mathrm{Cr}(\mathrm{vI})$ to an unknown intermediate chromium species (chromatograms B3 and B4 of Fig. 3) with the same retention time as the $\mathrm{Cr}(\mathrm{III})$ intermediate was observed when stored frozen at $-20{ }^{\circ} \mathrm{C}$. Instability of $\mathrm{Cr}$ (III) was observed in all storage conditions (see Fig. 3). The $\mathrm{pH}$ of water standards is neutral, so it is possible that the unknown $\mathrm{Cr}(\mathrm{III})$ intermediate observed in chromatograms (A3) and (A4) of Fig. 3 is a result of the incomplete hydrolysis or dissociation of the trivalent chromium compound $\mathrm{Cr}\left(\mathrm{H}_{2} \mathrm{O}\right)_{6}{ }^{3+}$ to $\mathrm{Cr}(\mathrm{OH})^{2+}, \mathrm{Cr}(\mathrm{OH})_{2}{ }^{+}$or $\mathrm{Cr}(\mathrm{OH})_{3}$. Publications concerned with the determination of trace elements in EBC, specify that samples of EBC were frozen at $-80{ }^{\circ} \mathrm{C}$ after collection. ${ }^{20,21,24,25,46}$ Initial observations of freezing a spiked aqueous standard of chromium $\mathrm{Cr}(\mathrm{III})$ and $\mathrm{Cr}(\mathrm{VI})$ at $-80{ }^{\circ} \mathrm{C}$ showed the same pattern of conversion to intermediate species as the aqueous standard stored at $-20^{\circ} \mathrm{C}$ for 24 hours. If $\mathrm{Cr}(\mathrm{vI})$ cannot be successfully stored frozen, chromium speciation samples would need to be stored under refrigeration. Cr(III) cannot be successfully stored at any temperature so the complexation with EDTA is needed for stability.

2. Derivatisation by complexation of $\mathrm{Cr}$ (III) with EDTA. The first thing to note is that by complexing $\operatorname{Cr}$ (III) with EDTA the elution order of the two chromium species changes. EDTA does not form a complex with $\mathrm{Cr}(\mathrm{VI})$. Without complexation, $\mathrm{Cr}(\mathrm{III})$ elutes after $\mathrm{Cr}(\mathrm{vI})$ with a retention time of approximately 4.5 minutes. $\mathrm{Cr}$ (III) in solution exists in the form of a cationic hydroxy complex and is retained on the sulfonated region of the column whilst the $\mathrm{Cr}(\mathrm{vI})$ ions are retained on the alkyl quaternary ammonium region. However after complexation the
EDTA-Cr(III) complex elutes first with a retention time of just over 1 minute in the final optimised method, indicating the anionic EDTA-Cr(III) ions are no longer retained by the column.

The chromatograms in Fig. 4 show that the complexation reaction of $\mathrm{Cr}$ (III) with the EDTA solution was not instantaneous but achieved near full complexation after one hour at room temperature prior to analysis. In many of the studies published where EDTA complexation was used to stabilise or change the charge of $\mathrm{Cr}(\mathrm{III})$, concentrations lower than $0.5 \mathrm{mM}$ EDTA were used and heat was also used for the derivatisation reaction to occur. Both Suzuki \& Serita ${ }^{41}$ and Byrdy et al. $^{39}$ heated the samples in a heat block for 1 hour at $50{ }^{\circ} \mathrm{C}$; Gurleyuk \& Wallschlager ${ }^{40}$ heated their samples for 1 hour at $70{ }^{\circ} \mathrm{C}$ and Leist et al. ${ }^{47}$ heated for 1.5 hours at $70^{\circ} \mathrm{C}$. This approach would not be practical after the collection of EBC samples from workers at a place of work, and samples may not be delivered to the laboratory for 24 hours, meaning immediate heating of samples after collection in a heat block is not a viable option. However, Wolf $e t$ al. ${ }^{27}$ found that the complexation reaction was fully achieved with $0.5 \mathrm{mM}$ of EDTA when the samples were left to stand at room temperature for 30 minutes prior to analysis; having adjusted the $\mathrm{pH}$ of the EDTA solution to $\mathrm{pH}$ 7.6. The $\mathrm{pH}$ of the EDTA solution used in this study without any adjustment was $\mathrm{pH} 5$, which may explain why the complexation reaction shown in chromatogram B of Fig. 4 was not $100 \%$ effective.

3. Effect of $\mathbf{p H}$ on the EDTA-Cr(III) complex and $\mathrm{Cr}(\mathrm{vI})$. Having undertaken the experiment outlined in Section 2 of the method development - Derivatisation by complexation of $\mathrm{Cr}(\mathrm{III})$ with EDTA, it was necessary to determine the effect of $\mathrm{pH}$ on the ability of EDTA to successfully complex $\mathrm{Cr}$ (III). It can be seen in Fig. 5 that EDTA at pH 4, 5 and 7 did not fully complex and

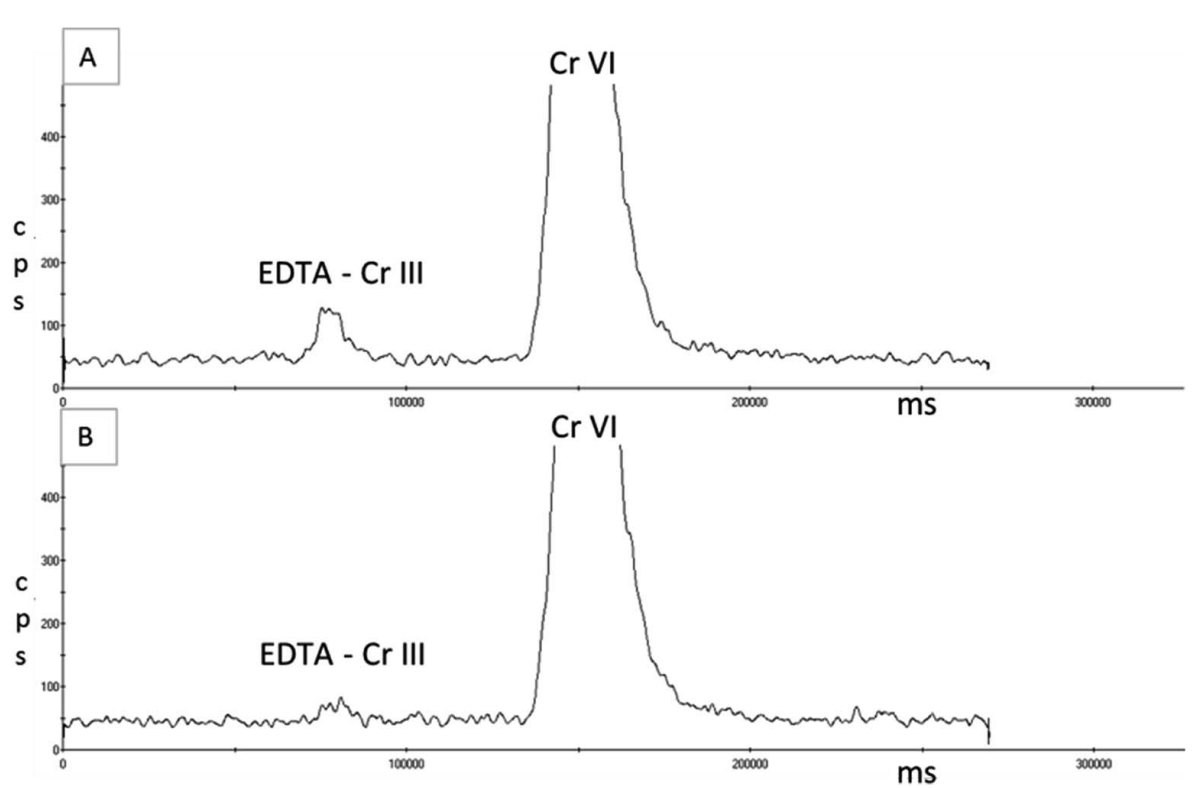

Fig. 7 Chromatograms of two $\mathrm{Cr}(\mathrm{VI})$ spiked EBC samples, stored at fridge temperature $\left(\sim 4{ }^{\circ} \mathrm{C}\right)$ for 6 weeks prior to analysis using an ESI OneFAST system coupled to a Dionex AG7 anion exchange column and ICP-MS using a nitric acid and ammonia mixed mobile phase. (A) EDTA pH 7.6. (B) EDTA pH 8 
stabilise $\mathrm{Cr}(\mathrm{III})$, meaning the elution of $\mathrm{Cr}$ (III) resulted in two peaks, whereas EDTA at pH 7.6, 8 and 9 show full complexation of $\mathrm{Cr}$ (III). The retention time of the EDTA-Cr(III) complex is the same throughout the $\mathrm{pH}$ range, however $\mathrm{pH}$ does affect the efficiency of complexation. The pH of the EDTA solution in the study reported by Wolf $e t$ al. $^{27}$ is within a range of $\mathrm{pH}$ 7.2-7.6.

Single standards of $5 \mu \mathrm{g} \mathrm{L}^{-1} \mathrm{Cr}(\mathrm{vI})$ were prepared in the three $\mathrm{pH}$ solutions of EDTA that achieved full complexation of $\mathrm{Cr}(\mathrm{III})$; pH 7.6, 8 and 9. Fig. 6 shows that $\mathrm{pH} 7.6$ and 8 had no effect on $\mathrm{Cr}(\mathrm{vI})$; however peak splitting was observed at $\mathrm{pH} 9$.

4. Concentration of EDTA. As $\mathrm{pH}$ is an important factor in the complexation of EDTA with $\mathrm{Cr}(\mathrm{III})$, it was necessary to determine if the increased concentration of EDTA was also an important factor. As both $\mathrm{pH} 7.6$ and $\mathrm{pH} 8$ have both shown to be a suitable $\mathrm{pH}$ for the EDTA solution, $\mathrm{pH} 8$ was selected for the $\mathrm{pH}$ of the three different concentrations of EDTA solutions. The varying concentrations of $0.5,0.6$ and $0.7 \mathrm{mM}$ EDTA solution at pH 8 made no difference to the retention, sensitivity or peak shape of either chromium species (data not shown).

5. Storage stability of EBC samples. Having developed the chromium speciation method, storage stability studies of $\mathrm{Cr}(\mathrm{III})$ and $\mathrm{Cr}(\mathrm{vI})$ in EBC samples were undertaken. Analysis of EBC spiked with single standards of $\mathrm{Cr}(\mathrm{III})$ and $\mathrm{Cr}(\mathrm{VI})$ at a concentration of $5 \mu \mathrm{g} \mathrm{L} \mathrm{L}^{-1}$ diluted 1 in 10 with a $0.5 \mathrm{mM}$ EDTA solution of either $\mathrm{pH} 7.6$ or $\mathrm{pH} 8$ showed that the EBC EDTA-Cr(III) complex was stable with no sign of deterioration or species conversion for the entire 6 week period at both $\mathrm{pH}$ values. The $\mathrm{Cr}(\mathrm{vr})$ sample was also stable for the 6 week storage period when the $\operatorname{Cr}(\mathrm{vI})$
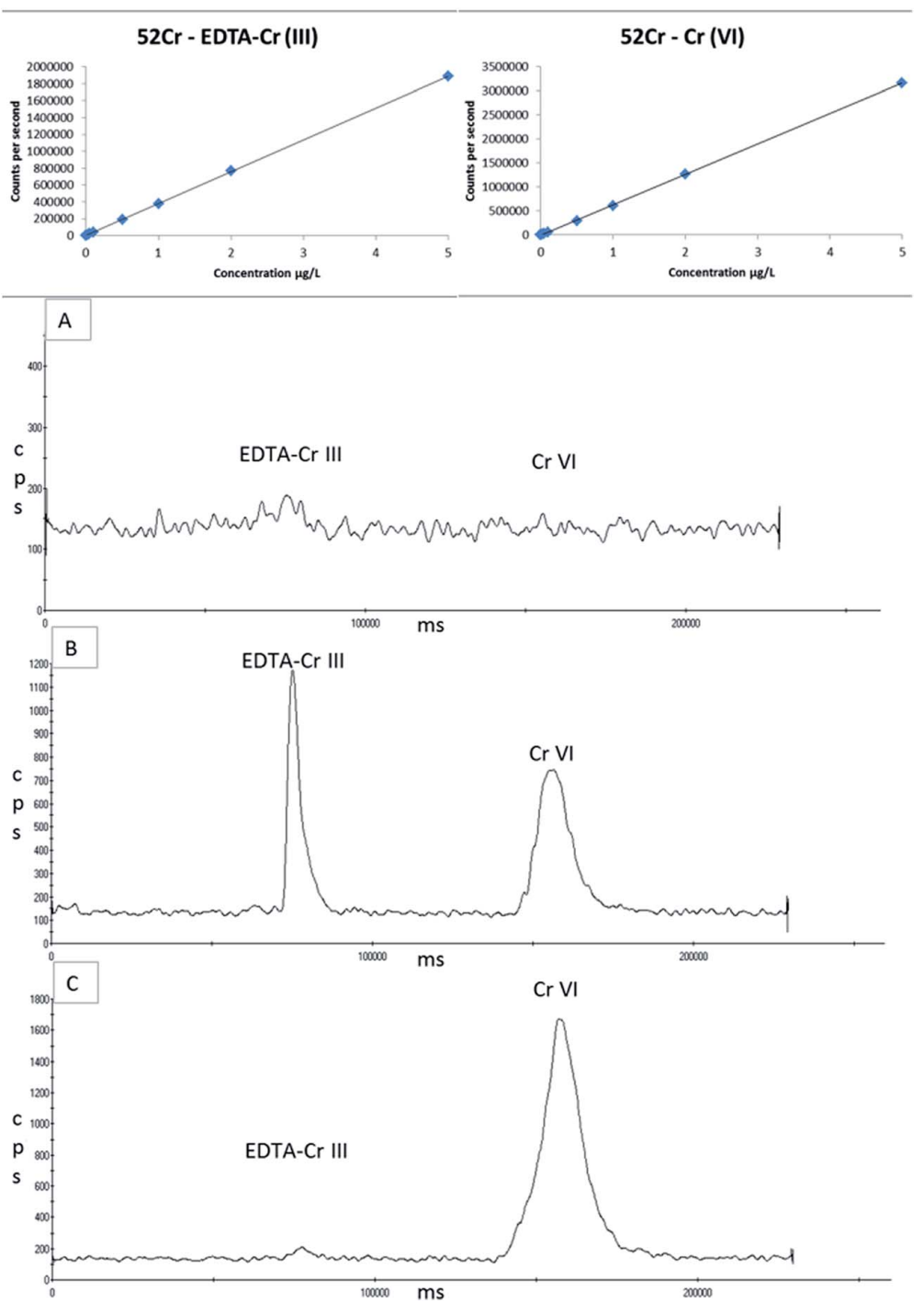

Fig. 8 Linear calibration curves of $0-5 \mu \mathrm{g} \mathrm{L}^{-1}$ for both EDTA-Cr(III) and $\mathrm{Cr}(\mathrm{vI})$ using an ESI OneFAST system coupled to a Dionex AG7 anion exchange column and ICP-MS using a nitric acid and ammonia mixed mobile phase. (A) Blank EBC sample. (B) A $2 \mu \mathrm{g} \mathrm{L}{ }^{-1}$ spiked blank EBC sample with EDTA-Cr(III) and $\mathrm{Cr}(\mathrm{VI})$. (C) Commercially available proficiency testing material for $\mathrm{Cr}(\mathrm{VI})$ in drinking water. 
spiked EBC samples were diluted with the $\mathrm{pH} 8$ solution of EDTA. However the $\mathrm{Cr}(\mathrm{vI})$ spiked EBC samples that had been diluted with the pH 7.6 EDTA solution showed signs of instability after only 1 week of storage. At $\mathrm{pH} 7.6$ the $\mathrm{Cr}(\mathrm{vI})$ spiked EBC sample began to show a peak at the retention time of EDTA-Cr(III) and this had increased in size when analysed at 4 weeks and again at 6 weeks showing a $6 \%$ recovery of EDTA$\mathrm{Cr}(\mathrm{III})$ in an $\mathrm{EBC} \mathrm{Cr}(\mathrm{VI})$ only sample whereas the $\mathrm{pH} 8$ solution showed no EDTA-Cr(III) at 6 weeks (see Fig. 7). The pH of undiluted EBC was seen to range from $\mathrm{pH}$ 6-6.5 and, although the prepared EBC was diluted 1 in 10 with the EDTA solution adjusted to $\mathrm{pH} 7.6$, the final $\mathrm{pH}$ of the dilution would be less than $\mathrm{pH}$ 7.6. Wolf et al. ${ }^{27}$ determined that when the $\mathrm{pH}$ of their EDTA/TBAOH solution was less than pH 7.4 more than $10 \%$ of their $\mathrm{Cr}(\mathrm{vI})$ samples were present as $\mathrm{HCrO}_{4}^{-}$and when it dropped to between $\mathrm{pH} 6$ and $\mathrm{pH} 7$ their $\mathrm{Cr}(\mathrm{vI})$ sample existed as $\mathrm{HCrO}_{4}{ }^{2-}$. Either the EBC Cr(vI) sample here is slowly reducing to $\mathrm{Cr}(\mathrm{III})$ in the neutral to acidic EDTA solution during storage or it is slowly converting to a different compound of $\mathrm{Cr}(\mathrm{vI})$ which has the same retention time as EDTA-Cr(III). It is neither appropriate nor practical to measure the $\mathrm{pH}$ of each person's EBC sample and adjust accordingly when work place monitoring, so an EDTA solution at $\mathrm{pH} 8$ was chosen to compensate for any reduction in $\mathrm{pH}$ as a result of the EBC.

6. Optimised method. The chromatogram in Fig. 8 shows that this optimised method achieves full separation of EDTA$\mathrm{Cr}$ (III) and $\mathrm{Cr}(\mathrm{VI})$ in 3 minutes $(180000 \mathrm{~ms})$. The calibration range used in this study was $0-5 \mu \mathrm{g} \mathrm{L}^{-1}$ for both species of chromium. The limit of detection (LOD) was calculated as three times the standard deviation of the blank; this was $0.006 \mu \mathrm{g} \mathrm{L}^{-1}$ and $0.002 \mu \mathrm{g} \mathrm{L}^{-1}$ for $\mathrm{Cr}(\mathrm{III})$ and $\mathrm{Cr}(\mathrm{VI})$ respectively. The limit of quantification (LOQ) was calculated as 10 times the mean background equivalent concentration (BEC); this was $0.040 \mu \mathrm{g} \mathrm{L}^{-1}$ and $0.013 \mu \mathrm{g} \mathrm{L}^{-1}$ for $\mathrm{Cr}(\mathrm{III})$ and $\mathrm{Cr}(\mathrm{vI})$ respectively.

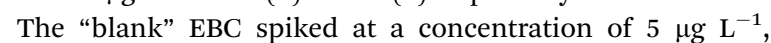
analysed on four separate occasions over the 6 week storage period, gave an average recovery of $91 \% \pm 0.13$ for $\mathrm{Cr}(\mathrm{III})$ and $95 \% \pm 0.02$ for $\mathrm{Cr}(\mathrm{vI})$. The proficiency testing material when prepared 1 in 1000 for this study has a target value of $2.81 \mu \mathrm{g} \mathrm{L}^{-1}$ and an acceptance range of 2.28-3.28 $\mu \mathrm{g} \mathrm{L}^{-1}$. The PTM sample was analysed three times and gave an average within range result of $2.65 \pm 0.03 \mu \mathrm{g} \mathrm{L}^{-1}$ for $\mathrm{Cr}(\mathrm{vI})$.

Calibration standards, samples and quality control material are diluted 10 fold with $0.5 \mathrm{mM}$ EDTA solution adjusted to $\mathrm{pH} 8$ with $10 \% \mathrm{v} / \mathrm{v}$ ammonia solution. These samples are left to stand a minimum of 1 hour prior to analysis $\mu$ LC-ICP-MS, and can be stored refrigerated for up to 6 weeks.

The use of ICP-MS can result in spectroscopic interferences, e.g. with polyatomic interferences ${ }^{40} \mathrm{Ar}^{12} \mathrm{C}^{+}$and ${ }^{36} \mathrm{Ar}^{16} \mathrm{O}^{+}$on ${ }^{52} \mathrm{Cr}$. However the optimised use of the collision cell will avoid most of these interferences. It is also possible that the sample matrix could create additional interferences given that element such as $\mathrm{C}, \mathrm{O}$ and $\mathrm{Cl}$ are abundant in biological samples. The main contributor to a potential high background signal intensity on ${ }^{52} \mathrm{Cr}$ due to ${ }^{40} \mathrm{Ar}^{12} \mathrm{C}^{+}$. Having injected a weak butanol solution with and without the EBC matrix, no peak or increase in background signal intensity was observed. The main chlorine based interference on ${ }^{52} \mathrm{Cr}$ would be the ${ }^{35} \mathrm{Cl}^{16} \mathrm{O}^{1} \mathrm{H}^{+}$. To investigate the impact of a chloride interference on the chromium species a weak solution of $\mathrm{HCl}$ was injected onto the column. Prior to complexing $\mathrm{Cr}(\mathrm{III})$ with EDTA, Cl eluted before $\mathrm{Cr}(\mathrm{VI})$ and $\mathrm{Cr}(\mathrm{III})$. However, after complexing Cr(III) with EDTA and altering the retention time, when a weak solution of $\mathrm{HCl}$ is injected onto the column or added to 'blank' EBC, $\mathrm{Cl}$ and EDTA-Cr(III) elute at the same time. However, unlike a urine sample, the chloride content in EBC is negligible, as can be seen in the blank EBC in Fig. 8a. Furthermore the recovery of the spiked sample for $\mathrm{Cr}$ (III) in the stability study was $91 \%$ so no over recovery from an interference is evident. To confirm the absence of a chloride interference, two EBC samples were collected from five volunteers who were not occupationally exposed to chromium. There was no significant peaks obtained at $\mathrm{Cr}(\mathrm{III})$ in fact both EDTA$\mathrm{Cr}(\mathrm{III})$ and $\mathrm{Cr}(\mathrm{VI})$ were less than the LOQ for all $10 \mathrm{EBC}$ samples analysed.

\section{Conclusion}

The method described in this study is a robust and sensitive method for the simultaneous determination of $\mathrm{Cr}(\mathrm{III})$ and $\mathrm{Cr}(\mathrm{VI})$ in an EBC sample. This speciation method has been validated with spiked samples of blank EBC and a commercially available drinking water proficiency testing material sample for $\mathrm{Cr}(\mathrm{vI})$. It has been demonstrated that water samples and EBC samples containing $\mathrm{Cr}(\mathrm{III})$ and $\mathrm{Cr}(\mathrm{vI})$ can be stored in the fridge $\left(\sim 4^{\circ} \mathrm{C}\right)$ for up to 6 weeks when they have been diluted 10 fold with the EDTA solution, and that EBC samples must not be frozen after collection if the integrity of $\mathrm{Cr}(\mathrm{vI})$ is to be maintained. This study has developed a practical approach to collecting EBC samples in the workplace from workers who are potentially occupationally exposed to hexavalent chromium and that the integrity of the chromium species will be intact prior to analysis by $\mu \mathrm{LC}-\mathrm{ICP}-$ MS. The next stage of this study will be to undertake site visits to collect EBC samples from chromium workers and apply the methods developed here to these 'real' samples.

\section{Acknowledgements}

This publication and the work it describes were funded by the Health and Safety Executive (HSE). Its contents, including any opinions and/or conclusions expressed, are those of the authors alone and do not necessarily reflect HSE policy.

\section{References}

1 G. F. Nordberg, B. A. Fowler, M. Nordberg and L. T. Friberg, Handbook on the toxicology of metals, Elsevier Academic Press, London, 3rd edn, 2007.

2 Agents classified by the IARC monographs, volumes 1-113, http://monographs.iarc.fr/ENG/Classification/latest_classif.php (Accessed September 2015).

3 R. Rakhunde, L. Deshpande and H. D. Juneja, Crit. Rev. Environ. Sci. Technol., 2012, 42(7), 776-810.

4 IARC monographs on the evaluation of carcinogenic risks to humans, Volume 100 C. A review of human carcinogens: 
arsenic, metals, fibres and dusts. http://monographs.iarc.fr/ ENG/Monographs/vol100C/index.php (accessed September 2015).

5 D. J. Paustenbach, B. L. Finley, F. S. Mowat and B. D. Kerger, J. Toxicol. Environ. Health, Part A, 2003, 66(14), 1295-1339.

6 F. S. De, D. Serra, A. Camoirano and P. Zanacchi, Biol. Trace Elem. Res., 1989, 21, 179-187.

7 P. Hoet, in Handbook of elemental speciation II: Species in the environment, food, medicine and occupational health, ed. R. Cornelis, J. Caruso, H. Crews and K. Heumann, WileyBlackwell, 2005, ch. 2.6.2, pp. 136-157.

8 A. Zhitkovich, Chem. Res. Toxicol., 2011, 24(10), 1617-1629.

9 HSL, Guidance on laboratory techniques in occupational medicine, HSE, 12th edn, 2013.

10 DFG German DRF Deutsche Forschungsgemeinschaft, List of MAK and BAT values 2009, Wiley-VCH, Germany, 2012.

11 American Conference of Governmental Industrial Hygienists, TLVS and BEIs: Threshold limit values for chemical substances and physical agents, Signature Publications, USA, 2015.

12 G. E. Corbett, D. G. Dodge, E. O'Flaherty, J. Liang, L. Throop, B. L. Finley and B. D. Kerger, Environ. Res., 1998, 78(1), 7-11.

13 J. P. Goulle, E. Saussereau, J. Grosjean, C. Doche, L. Mahieu, J. M. Thouret, M. Guerbet and C. Lacroix, Forensic Sci. Int., 2012, 217(1-3), 8-12.

14 M. L. Chen, Y. N. Zhao, D. W. Zhang, Y. Tian and J. H. Wang, J. Anal. At. Spectrom., 2010, 25(11), 1688-1694.

15 Y. M. Scindia, A. K. Pandey, A. R. Reddy and S. B. Manohar, Anal. Chim. Acta, 2004, 515(2), 311-321.

16 A. S. Stasinakis, N. S. Thomaidis and T. D. Lekkas, Anal. Chim. Acta, 2003, 478(1), 119-127.

17 L. Girard and J. Hubert, Talanta, 1996, 43(11), 1965-1974.

18 M. Corradi and A. Mutti, Acta Biol. Med., 2005, 76(2), 20-29.

19 S. A. Kharitonov and P. J. Barnes, Am. J. Respir. Crit. Care Med., 2001, 163(7), 1693-1722.

20 M. Goldoni, M. Corradi, P. Mozzoni, G. Folesani, R. Alinovi, S. Pinelli, R. Andreoli, D. Pigini, R. Tillo, A. Filetti, C. Garavelli and A. Mutti, J. Breath Res., 2013, 7(1), 017101.

21 M. Goldoni, S. Catalani, G. De Palma, P. Manini, O. Acampa, M. Corradi, R. Bergonzi, P. Apostoli and A. Mutti, Environ. Health Perspect., 2004, 112(13), 1293-1298.

22 H. C. Broding, B. Michalke, T. Goen and H. Drexler, Int. Arch. Occup. Environ. Health, 2009, 82(5), 565-573.

23 F. Hoffmeyer, T. Weiss, M. Lehnert, B. Pesch, H. Berresheim, J. Henry, M. Raulf-Heimsoth, C. H. Broding, J. Buenger, V. Harth and T. Bruening, J. Environ. Monit., 2011, 13(1), 212-218.

24 A. Caglieri, M. Goldoni, O. Acampa, R. Andreoli, M. V. Vettori, M. Corradi, P. Apostoli and A. Mutti, Environ. Health Perspect., 2006, 114(4), 542-546.

25 S. Hulo, N. Cherot-Kornobis, M. Howsam, S. Crucq, V. de Broucker, A. Sobaszek and J. L. Edme, Toxicol. Lett., 2014, 226(1), 63-69.
26 M. Goldoni, A. Caglieri, D. Poli, M. V. Vettori, M. Corradi, P. Apostoli and A. Mutti, Anal. Chim. Acta, 2006, 562(2), 229-235.

27 R. E. Wolf, J. M. Morrison and M. B. Goldhaber, J. Anal. At. Spectrom., 2007, 22(9), 1051-1060.

28 J. Scancar and R. Milacic, J. Anal. At. Spectrom., 2014, 29(3), 427-443.

29 K. Neubauer, W. Reuter and P. Perrone, Application Note, Perkin Elmer Life and Analytical Sciences, USA, 2003.

30 C. Puls and A. Limbeck, J. Anal. At. Spectrom., 2009, 24(10), 1434-1440.

31 M. M. L. Guerrero, E. V. Alonso, J. M. C. Pavon, M. T. S. Cordero and A. G. de Torres, J. Anal. At. Spectrom., 2012, 27(4), 682-688.

32 M. J. Marques, A. Morales-Rubio, A. Salvado and M. de la Guardia, Talanta, 2001, 53(6), 1229-1239.

33 J. Prokisch, S. A. Katz, B. Kovacs and Z. Gyori, J. Chromatogr. A, 1997, 774(1-2), 363-371.

34 R. P. Monasterio, G. E. Lascalea, L. D. Martinez and R. G. Wuilloud, J. Trace Elem. Med. Biol., 2009, 23(3), 157166.

35 M. Sperling, S. K. Xu and B. Welz, Anal. Chem., 1992, 64(24), 3101-3108.

36 B. H. Li and X. P. Yan, Electrophoresis, 2007, 28(9), 13931398.

37 Z. Chen, M. Megharaj and R. Naidu, Talanta, 2007, 72(2), 394-400.

38 M. Pantsar-Kallio and P. Manninen, Anal. Chim. Acta, 1996, 318(3), 335-343.

39 F. A. Byrdy, L. K. Olson, N. P. Vela and J. A. Caruso, J. Chromatogr. A, 1995, 712(2), 311-320.

40 H. Gurleyuk and D. Wallschlager, J. Anal. At. Spectrom., 2001, 16(9), 926-930.

41 Y. Suzuki and F. Serita, Ind. Health., 1985, 23(3), 207-220.

42 F. Seby, S. Charles, M. Gagean, H. Garraud and O. F. X. Donard, J. Anal. At. Spectrom., 2003, 18(11), 13861390.

43 C. Barnowski, N. Jakubowski, D. Stuewer and J. A. C. Broekaert, J. Anal. At. Spectrom., 1997, 12(10), 11551161.

44 L. Xing and D. Beauchemin, J. Anal. At. Spectrom., 2010, 25(7), 1046-1055.

45 M. Goldoni, A. Caglieri, G. De Palma, O. Acampa, P. Gergelova, M. Corradi, P. Apostoli and A. Mutti, J. Environ. Monit., 2010, 12(2), 442-447.

46 M. Gube, J. Ebel, P. Brand, T. Goeen, K. Holzinger, U. Reisgen and T. Kraus, Int. Arch. Occup. Environ. Health, 2010, 83(7), 803-811.

47 M. Leist, R. Leiser and A. Toms, The Application NotebookAdvertising Supplement Mass Spectrometry, Varian Inc., 2006, pp. 29-31. 


\section{Chapter 5.}

The Simultaneous Detection of Trivalent and Hexavalent Chromium in Exhaled Breath Condensate: A Feasibility Study Comparing Workers and Controls.

E. Leese, J. Morton, P.H.E. Gardiner and V.A. Carolan. Int. J. Hyg. Environ. Health, 2017, $220,415-423$.

Published - Government Open Access Licence

http://www.sciencedirect.com/science/article/pii/S1438463916302620 


\title{
The simultaneous detection of trivalent \& hexavalent chromium in exhaled breath condensate: A feasibility study comparing workers and controls
}

\author{
Elizabeth Leese ${ }^{\mathrm{a}, \mathrm{b}, *}$, Jackie Morton ${ }^{\mathrm{a}}$, Philip H.E. Gardiner ${ }^{\mathrm{b}}$, Vikki A. Carolan ${ }^{\mathrm{b}}$ \\ ${ }^{a}$ Health and Safety Executive, Harpur Hill, Buxton, Derbyshire SK17 9JN, UK \\ b Biomolecular Sciences Research Centre, Sheffield Hallam University, Howard Street, Sheffield S1 1WB, UK
}

\section{A R T I C L E I N F O}

\section{Article history:}

Received 24 August 2016

Received in revised form 7 November 2016

Accepted 1 December 2016

\section{Keywords:}

Chromium

Hexavalen

Trivalent

Exhaled breath condensate

$\mu$ LC-ICP-MS

Occupational exposure

\begin{abstract}
A B S T R A C T
The analytical method outlined in this feasibility study has been used to show that trivalent chromium $(\mathrm{Cr}(\mathrm{III}))$ and hexavalent chromium $(\mathrm{Cr}(\mathrm{VI}))$ can be detected and measured in exhaled breath condensate (EBC) samples. EBC samples and urine samples were collected from a cohort of 58 workers occupationally exposed to hexavalent chromium compounds and 22 unexposed volunteers (control group). Levels of $\mathrm{Cr}(\mathrm{III})$ and $\mathrm{Cr}(\mathrm{VI})$ were determined in $\mathrm{EBC}$ samples and total chromium levels were determined in urine samples. Pre and post working week samples for both EBC and urine were collected in tandem. Total chromium in urine samples was analysed by inductively coupled plasma mass spectrometry (ICP-MS). Analysis of $\mathrm{Cr}(\mathrm{III})$ and $\mathrm{Cr}(\mathrm{VI})$ in EBC samples used a hyphenated micro liquid chromatography $(\mu \mathrm{LC})$ system coupled to an ICP-MS. Separation was achieved using an anion exchange micro-sized column. The results showed that the occupationally exposed workers had significantly higher levels of $\mathrm{Cr}(\mathrm{III})$ and $\mathrm{Cr}(\mathrm{VI})$ in their EBC samples than the control group, as well as higher levels of total chromium in their urine samples. However, for the exposed workers no significant difference was found between pre and post working week EBC samples for either $\mathrm{Cr}(\mathrm{III})$ or $\mathrm{Cr}(\mathrm{VI})$. This study has established that $\mathrm{Cr}(\mathrm{III})$ and $\mathrm{Cr}(\mathrm{VI}) \mathrm{can}$ simultaneously be detected and measured in 'real' EBC samples and will help in understanding inhalation exposure.
\end{abstract}

Crown Copyright @ 2016 Published by Elsevier GmbH. This is an open access article under the Open Government License (OGL) (http://www.nationalarchives.gov.uk/doc/open-government-licence/ version/3/).

\section{Introduction}

In Great Britain (GB), many activities/occupations can involve the use of chemicals considered hazardous to worker health. It is important that exposure to chemicals which are classified as carcinogens, mutagens and sensitisers be reduced to as low as reasonably practicable as stated in legislation (Health \& Safety Executive (HSE), 2013a). Biological monitoring (the measurement of a chemical or its breakdown products in a biological sample) is

Abbreviations: EBC, exhaled breath condensate; $\mathrm{Cr}(\mathrm{III})$, trivalent chromium; $\mathrm{Cr}(\mathrm{VI})$, hexavalent chromium; ICP-MS, inductively coupled plasma mass spectrometry; $\mu \mathrm{LC}$, micro liquid chromatography; $\mathrm{COSHH}$, control of substances hazardous to health; ATSDR, agency for toxic substances and disease registry; IARC, international agency for research on cancer; HSE, Health \& Safety Executive; RBC, red blood cells: HSL, Health and Safety Laboratory; BMGV, biological monitoring guidance value. * Corresponding author at: Health \& Safety Executive, Harpur Hill, Buxton SK17 9JN, UK.

E-mail address: Liz.leese@hsl.gsi.gov.uk (E. Leese). one approach for assessing occupational exposure to a chemical in the workplace.

Hexavalent chromium ( $\mathrm{Cr}(\mathrm{VI}))$, which includes chromates, dichromates and chromic acid is one such chemical that is classified as both a sensitising agent and a carcinogen (Agency for toxic substances and disease registry (ATSDR), 2012; International agency for research on cancer (IARC), 2016)). Exposure to $\mathrm{Cr}(\mathrm{VI})$ compounds in the workplace can be through inhalation directly into the lungs from breathing in dust, fumes or mists; dermal absorption through the skin by contact with chromium solutions or solids; or by ingestion as a result of hand to mouth contamination from handling food, smoking or biting nails when hands are contaminated with chromium dust or solutions. Exposure to $\mathrm{Cr}(\mathrm{VI})$ compounds can cause respiratory irritations such as nosebleeds, ulcers and holes in the nasal septum, inflammatory respiratory problems, skin irritation and rashes from allergic dermatitis, upset stomachs, kidney and liver damage along with lung and nasal cancer (ATSDR, 2012; HSE, 2013b; Rakhunde et al., 2012). The types of industry and commercial processes where occupational expo- 
sure to $\mathrm{Cr}(\mathrm{VI})$ compounds might occur are chromate production, production of chromate pigments and dyes, electroplating and anodising, the production of stainless steel and other chromium alloys, the cutting, finishing and welding of chromium alloys and stainless steel, leather tanning and the production and spraying of paints used in the aeronautic and maritime industries (Genovese et al., 2015; HSE, 2013b; Rakhunde et al., 2012).

Exposure to $\mathrm{Cr}(\mathrm{VI})$ compounds leads to accumulation in various tissues such as the lungs, liver and kidneys (Genovese et al., 2015), with glutathione and ascorbate being responsible for reduction of $\mathrm{Cr}(\mathrm{VI})$ to trivalent chromium ( $\mathrm{Cr}(\mathrm{III})$ ) within cells with the aid of cysteine (De Flora et al., 1989; Paustenbach et al., 2003). $\mathrm{Cr}(\mathrm{III})$ is an essential element as it is needed for the regulation and metabolism of glucose, sugars and fats (Genovese et al., 2015) and thus is considered non-toxic. This difference in toxicity between the two chromium species is largely related to their permeability into cells. Although $\mathrm{Cr}(\mathrm{III})$ is more reactive than $\mathrm{Cr}(\mathrm{VI})$ (e.g. binding to DNA), $\mathrm{Cr}$ (III) is unable to permeate cell membranes, $\mathrm{Cr}$ (VI) however is easily taken up by cells; where it undergoes sequential reduction from $\mathrm{Cr}(\mathrm{V})$ to $\mathrm{Cr}$ (IV) and finally $\mathrm{Cr}$ (III) (De Flora et al., 1989; Hoet, 2005). Although exposure to $\mathrm{Cr}(\mathrm{VI})$ can be measured in red blood cells (RBC) determined as $\mathrm{Cr}(\mathrm{III})$, reduction of $\mathrm{Cr}(\mathrm{VI})$ to $\mathrm{Cr}(\mathrm{III})$ primarily occurs in plasma, reducing the uptake of $\mathrm{Cr}(\mathrm{VI})$ into RBC (Hoet, 2005). For the purposes of biological monitoring for exposure assessment, a urine sample is widely accepted, largely due to it being less invasive and easier to collect than a blood sample. All detectable chromium in a urine sample will be in the form of $\mathrm{Cr}$ (III) (De Flora, 2000) and although this is not an ideal approach to $\mathrm{Cr}(\mathrm{VI})$ exposure assessment, it is a practical one and widely used. A background unexposed reference range of $<2.9 \mu \mathrm{mol} / \mathrm{mol}$ creatinine (Morton et al., 2014) has been established by HSE's, Health and Safety Laboratory, and a Biological Monitoring Guidance Value (BMGV) of $10 \mu \mathrm{mol} / \mathrm{mol}$ creatinine has been determined, based on a Health \& Safety Executive (HSE) study where the 90th percentile was adopted from workplaces deemed to have good control (HSE, 2011). Urinary chromium levels can reflect both past and recent exposure, with daily accumulation through the working week of the occupationally exposed (Hoet, 2005). Studies have suggested the excretion of chromium in urine after exposure by inhalation as a two or three stage process (Hoet, 2005; Petersen et al., 2000), with elimination half-lives of $7 \mathrm{~h}, 15-30$ days and $3-5$ years (ATSDR, 2012; Hoet, 2005).

The main route of exposure to hexavalent chromium is by inhalation (De Flora, 2000; Hoet, 2005) so a suitable biological matrix to represent exposure by inhalation to hexavalent chromium compounds might be exhaled breath condensate (EBC). After an inhalation exposure of chromium into the lungs, it is partially removed by mucociliary clearance, but some is known to remain (Hoet, 2005). The exact bioavailability of respirable chromium is not known, however animal studies have shown that $53-85 \%$ of respirable $\mathrm{Cr}(\mathrm{VI})$ is cleared from the lungs by absorption into the blood stream or by mucociliary clearance in comparison to only $5-30 \%$ of respirable $\mathrm{Cr}(\mathrm{III})$, with the rest remaining in the lungs (ATSDR, 2012). The collection and measurement of EBC is a promising technique primarily due to its non-invasiveness and the lack of interfering solutes found in other biological matrices. EBC is the collection of condensate from cooled exhaled breath during regular tidal breathing. This collected exhaled air contains mostly water vapour but also droplets of fluid from the respiratory tract (Corradi and Mutti, 2005), meaning EBC measurements will reflect markers and molecules found in the mouth, tracheobronchial system and the alveoli (Kharitonov and Barnes, 2001). EBC is reported to contain both volatile substances in the gaseous phase along with low volatile and non-volatile substances in the form of droplets originating from the epithelial lining fluid, as aerosol particles in EBC (Rosias, 2012). The non-volatile substances will include salts, proteins, lipids and environmental and/or occupational contaminants (Corradi and Mutti, 2005; Goldoni et al., 2013; Kharitonov and Barnes, 2001).

There are only a small number of studies concerning the relationship between EBC measurements and occupational exposure, where detectable levels of metals in EBC have been reported such as; for cobalt and tungsten (Broding et al., 2009; Goldoni et al., 2004), beryllium (Hulo et al., 2016), manganese (Hulo et al., 2014), lead (Felix et al., 2015) and iron, nickel, aluminium and chromium (Caglieri et al., 2006; Goldoni et al., 2010; Goldoni et al., 2006; Gube et al., 2010; Hoffmeyer et al., 2011). To date there are only two published studies where $\mathrm{Cr}(\mathrm{VI})$ was measured in EBC samples of workers (Goldoni et al., 2010; Goldoni et al., 2006), however both used a method combining solvent extraction with complexation with diphenylcarbazide and measurement performed by atomic absorption spectrometry. There are no publications measuring $\mathrm{Cr}(\mathrm{VI})$ by inductively coupled plasma - mass spectrometry (ICPMS) and there are no publications reporting the measurement of $\mathrm{Cr}$ (III) in EBC samples of workers or background populations.

We have previously validated and published our novel analytical methodology in which the two chromium species were determined in EBC samples (Leese et al., 2016). The objective of this feasibility study was to determine if both $\mathrm{Cr}(\mathrm{III})$ and $\mathrm{Cr}(\mathrm{VI})$ could be detected and measured in 'real' EBC samples. Having collected samples the aim was to determine whether there was a difference between the levels of $\mathrm{Cr}(\mathrm{III})$ and $\mathrm{Cr}(\mathrm{VI})$ found in $\mathrm{EBC}$ samples between a control group not occupationally exposed to chromium compounds and an occupational group who are potentially occupationally exposed to $\mathrm{Cr}(\mathrm{VI})$ compounds by inhalation. In addition, any difference in chromium EBC levels would be investigated between pre and post working week samples from the occupationally exposed volunteers.

\section{Experimental}

\subsection{Study group}

The occupationally exposed group $(n=58)$ consisted of 53 males and 5 females, all were over the age of 18 years, with a mean age of 45 years. Individuals volunteered in response to an email sent to companies who had previously sent urine samples to the Health \& Safety Laboratory for chromium analysis as part of a biological monitoring program. The occupationally exposed workers were categorised into three types of workers and termed $\mathrm{Cr}(\mathrm{VI})$ Workers, Non-Cr(VI) Workers and Other Workers;

Cr(VI) Workers - Individuals who work directly with chromium compounds (e.g. $\mathrm{Cr}(\mathrm{VI})$ platers, jiggers and blenders).

Non-Cr(VI) Workers - Individuals who did not work directly with chromium compounds, but worked alongside colleagues working directly with chromium (e.g. nickel, silver or copper electroplaters, maintenance).

Other Workers - Individuals who work neither with chromium nor alongside chromium workers (e.g. inspectors, administration staff, and managers).

Table 1 shows all the types of occupations detailed by the occupationally exposed workers and in which of the three worker categories they were placed in this study.

The volunteers in the control group $(n=22)$ consisted of 16 males and 6 females, who were not occupationally exposed to $\mathrm{Cr}(\mathrm{VI})$ compounds. All were over the age of 18 years, with a mean age of 39 years. Individuals volunteered in response to an email request outlining the study, sent to staff at the Health \& Safety Laboratory.

The study was approved by the NHS Research Ethics Committee London - Camden \& Islington, REC number 14/LO/1273. All partici- 
Table 1

Types of occupational or workplace role listed on the questionnaire by all individual volunteers potentially exposed to occupational $\mathrm{Cr}(\mathrm{VI})$ compounds.

\begin{tabular}{l}
\hline Cr(VI) Workers \\
Chrome electroplater \\
Grinder \\
Plasma cutter \\
Blenders (chemical production of powders, liquids or solids) \\
Anodiser \\
Jigger \\
Passivater \\
Polisher \\
Non-Cr(VI) Workers (not working directly with chromium) \\
General area duties \\
Silver electroplating \\
Nickel electroplater \& electroless-plater \\
Copper plater \\
Zinc plater \\
Tin plater \\
Lab analyst \\
Other Workers \\
Production Team Leader \\
Inspector \\
Manager \\
Office worker \\
Supervisor \\
\hline
\end{tabular}

pating volunteers gave informed consent, and were provided with a participant information sheet. Volunteer information was collected via a questionnaire. Information collected included: gender, age and smoking status, and for the occupationally exposed group only; tasks and duties undertaken since providing the pre working week sample. Because this is a feasibility study only, information on length of employment status and instances of work related ill health were not obtained.

For the occupationally exposed workers, workplace site visits were conducted by the authors to collect both a urine and EBC sample from each volunteer on a Monday morning at the start of shift (pre working week) and again on a Thursday afternoon (post working week).

For the control group, urine samples and EBC samples were collected onsite at the Health and Safety Laboratory. Urine and EBC samples were collected from each control volunteer at the beginning of the week and again at the end of the working week as was done for the occupationally exposed group.

\subsection{Instrumentation}

All sample analysis was undertaken using inductively coupled plasma - mass spectrometry (ICP-MS). Both total chromium in urine and chromium species $\mathrm{Cr}(\mathrm{III})$ and $\mathrm{Cr}(\mathrm{VI})$ in EBC samples were determined using an XSERIES 2 ICP-MS (Thermo Fisher Scientific, Hemel Hempstead, UK) monitoring ${ }^{52} \mathrm{Cr}$. For both methods the ICPMS was operated in collision cell (CCT) mode using 7\% hydrogen in helium, RF power $1400 \mathrm{~W}$, using Ni Xt skimmer and sample cones. The ICP-MS conditions were optimised daily.

\subsubsection{EBC samples - chromium speciation}

Speciation analysis was performed using a hyphenated $\mu$ LC system with the XSERIES2 ICP-MS. The separation of $\mathrm{Cr}(\mathrm{III})$ and $\mathrm{Cr}(\mathrm{VI})$ was achieved using a $5 \mathrm{~cm}$ anion exchange column, (Dionex AG7 $4 \mathrm{~mm} \times 50 \mathrm{~mm}$ i.d., $10 \mu \mathrm{m}$, Thermo Fisher Scientific, Hemel Hempstead, UK). The delivery of both sample and mobile phase onto the column was accomplished using an ESI OneFAST system (Elemental Scientific, Warrington, UK), with the ICP-MS peristaltic pump controlling a constant flow rate of $1 \mathrm{~mL} / \mathrm{min}$, with a six port switching value and $500 \mu \mathrm{L}$ sample loop. The operating conditions of the $\mu$ LC-ICP-MS were: nebuliser gas flow rate $0.86-0.96 \mathrm{~L} / \mathrm{min}$ using a PFA-ST nebuliser (Elemental Scientific, Warrington, UK), collision gas flow rate $3.4-3.7 \mathrm{~mL} / \mathrm{min}$, dwell time of $100 \mathrm{~ms}$ for ${ }^{52} \mathrm{Cr}$. The specific conditions of the $\mu$ LC-ICP-MS method used in this study and the optimised conditions for the collection, stability and analysis of $\mathrm{Cr}(\mathrm{III})$ and $\mathrm{Cr}(\mathrm{VI})$ in $\mathrm{EBC}$ have been reported elsewhere (Leese et al., 2016).

\subsubsection{Urine samples - total chromium}

Analysis was performed on the XSERIES2 ICP-MS using a Burgener Miramist (Burgener Research Inc., Ontario, Canada) nebuliser. Operating conditions were: nebuliser gas flow rate $0.81-0.96 \mathrm{~L} / \mathrm{min}$, collision gas flow rate $3.5 \mathrm{~mL} / \mathrm{min}$, dwell times were $100 \mathrm{~ms}$ for ${ }^{52} \mathrm{Cr}$ and $20 \mathrm{~ms}$ for ${ }^{72} \mathrm{Ge}$ (internal standard), with 100 sweeps per replicate and three replicates per sample.

\subsection{Reagents}

Potassium dichromate $\left(\mathrm{K}_{2} \mathrm{Cr}_{2} \mathrm{O}_{7}\right)$ (analytical grade), chromium(III) chloride $\left(\mathrm{CrCl}_{3}\right)$ (laboratory grade) and EDTA (diaminoethanetetracetic acid) were obtained from Fisher Scientific (Loughborough, UK). SpA ammonia solution $\left(\mathrm{NH}_{3}\right)$ and UpA Nitric Acid were obtained from Romil (Cambridge, UK). BDH ICP-MS single standards of $1000 \mathrm{mg} / \mathrm{L}$ chromium and germanium were obtained from VWR International (Lutterworth, UK).

All solutions were prepared fresh each day of analysis using ultrapure deionised water $(18.2 \mathrm{M} \Omega \mathrm{hm} \mathrm{cm})$ from a Millipore system (Merck Millipore, Billerica, MA, USA).

\subsection{Sample collection}

All urine samples were collected in $25 \mathrm{~mL}$ urine collection bottles (Sterilin Ltd, Newport, UK). EBC samples were collected using a TURBO-DECCS (Transportable Unit for Research on Biomarkers Obtained from Disposable Exhaled Condensate Collection Systems) by ItalChill (Parma, Italy). The TURBO-DECCS technique is as previously described (Leese et al., 2016). Immediately after the collection of each EBC sample an aliquot of EBC was diluted 10 fold with the $0.5 \mathrm{mM}$ EDTA ( $\mathrm{pH}$ was adjusted to $\mathrm{pH} 8$ using $10 \% \mathrm{v} / \mathrm{v}$ aqueous ammonia solution) solution onsite by the authors. Urine and EBC samples were returned to the laboratory on the day of collection by the authors. Urine samples had creatinine content analysed upon arrival by the Jaffe alkaline picrate method (Cocker et al., 2011) on a Pentra 400 spectrophotometer (Horiba UK Ltd, Northampton, UK). Creatinine (a breakdown product of creatine phosphate in muscle, which is usually produced at a constant rate by the body) allows a urine sample to be normalised for hydration. Both urine samples and EBC samples were refrigerated at between 2 and $8^{\circ} \mathrm{C}$ until analysis.

\subsection{Sample preparation and analysis}

\subsubsection{EBC samples - chromium speciation}

A single mobile phase was prepared in water by adding $4 \%$ $\mathrm{v} / \mathrm{v}$ ammonia solution and $3.2 \% \mathrm{v} / \mathrm{v}$ ultrapure nitric acid $\mathrm{pH}$ was adjusted to between $\mathrm{pH} 1.8-2$ using the ammonia solution.

Exhaled breath condensate samples were allowed to reach room temperature and mixed on a roller mixer before analysis. The samples were analysed in duplicate. For the calibration standards, stock solutions of $1000 \mathrm{mg} / \mathrm{L}$ of $\mathrm{Cr}(\mathrm{III})$ and $\mathrm{Cr}(\mathrm{VI})$ were prepared in water. Using these $1000 \mathrm{mg} / \mathrm{L}$ stock solutions a $100 \mu \mathrm{g} / \mathrm{L}$ mixed species working solution was prepared in water. Using the $100 \mu \mathrm{g} / \mathrm{L}$ mixed species working solution, standards of $0.01,0.02,0.05,0.1,0.5,1$ and $2 \mu \mathrm{g} / \mathrm{L}$ were prepared in the $0.5 \mathrm{mM}$ EDTA solution, giving a linear calibration range up to $20 \mu \mathrm{g} / \mathrm{L}$. Samples above the linear calibration range were diluted further with the $0.5 \mathrm{mM}$ EDTA solution where necessary 
A commercially available proficiency testing material (PTM) was obtained from Sigma Aldrich (Dorset, $\mathrm{UK}$ ) for $\mathrm{Cr}(\mathrm{VI})$ in drinking water (sample 1045/PE1453, Lot LRAA1427). This PTM was within date with a certified range. Whilst the manufacturer's instructions suggest the PTM is diluted 100 fold in water, for the purpose of this study the PTM was diluted 1000 fold in water to more accurately reflect the levels of total chromium and $\mathrm{Cr}(\mathrm{VI})$ in $\mathrm{EBC}$ samples previously reported (Caglieri et al., 2006; Goldoni et al., 2010).

It was also necessary to collect a large volume of EBC from a volunteer not occupationally exposed to $\mathrm{Cr}(\mathrm{VI})$ to create an EBC blank sample and spike to use as an in-house quality control material. Three equal volumes of "blank" EBC were spiked to create three in-house controls: $2 \mu \mathrm{g} / \mathrm{L} \mathrm{Cr}(\mathrm{III}), 2 \mu \mathrm{g} / \mathrm{L} \mathrm{Cr}(\mathrm{VI})$ and a mixed $2 \mu \mathrm{g} / \mathrm{L}$ $\mathrm{Cr}(\mathrm{III})$ and $\mathrm{Cr}(\mathrm{VI}) \mathrm{EBC}$ sample.

Both the 1000 fold dilution of the PTM and the $2 \mu \mathrm{g} / \mathrm{L}$ in-house spiked EBC samples were diluted 10 fold with $0.5 \mathrm{mM}$ EDTA solution for analysis and refrigerated at between 2 and $8^{\circ} \mathrm{C}$ for up to 6 weeks between analyses, as per the storage stability study undertaken by Leese et al. (2016). The PTM and the spiked EBC samples were analysed at the beginning and the end of the analytical run as well as every 10 samples.

\subsubsection{Urine samples - total chromium}

For total chromium analysis, a single standard stock solution of $1 \mathrm{mg} / \mathrm{L}$ chromium was prepared in water from the $1000 \mathrm{mg} / \mathrm{L}$ single standard. Using the $1 \mathrm{mg} / \mathrm{L}$ stock solution, standards of $0.5,2.5,5$, 10 and $20 \mu \mathrm{g} / \mathrm{L}$ were prepared in water, giving a linear calibration up to $80 \mu \mathrm{g} / \mathrm{L}$.

Urine samples were allowed to reach room temperature and mixed on a roller mixer before analysis. Samples were diluted 20 fold and calibration standards 5 fold with a $1 \% \mathrm{v} / \mathrm{v}$ nitric acid solution containing $10 \mu \mathrm{g} / \mathrm{L} \mathrm{Ge} \mathrm{internal} \mathrm{standard,} \mathrm{following} \mathrm{the} \mathrm{Health} \mathrm{\&}$ Safety Laboratory UKAS accredited method for total chromium in urine. The certified reference materials (CRMs) were Lyphocheck urine metals control Level 1 (Lot 69161 \& 69171) (Bio-RAD Laboratories, Hemel Hempstead, UK) and Clinchek urine control for trace elements Level 2 (Lot 122 \& 432) (Recipe, Munich, Germany) and were analysed like the urine samples at the beginning and the end of each analytical run and after every 20 samples. In addition, a $2 \mu \mathrm{g} / \mathrm{L}$ standard was analysed at the beginning and end of each analytical run as well as every 10 samples to monitor drift within the analysis.

\section{Results}

\subsection{Analytical method E validation}

\subsubsection{EBC samples - chromium speciation}

The method described in this study is a sensitive and robust method for the determination of $\mathrm{Cr}(\mathrm{III})$ and $\mathrm{Cr}(\mathrm{VI})$ in $\mathrm{EBC}$ samples. The chromatograms in Fig. 1 show that this novel speciation method can reproducibly separate $\mathrm{Cr}(\mathrm{III})$ and $\mathrm{Cr}(\mathrm{VI})$ in both aqueous standards and an EBC matrix within 2 min 20 s (200,000 ms).

Based upon the results of twenty two analyses of the determination of chromium species in EBC samples, the limit of detection (LOD) and limit of quantification (LOQ) have been established for both $\mathrm{Cr}(\mathrm{III})$ and $\mathrm{Cr}(\mathrm{VI})$. The LOD were calculated as three times the standard deviation of the mean of the blank; these were $0.007 \mu \mathrm{g} / \mathrm{L}$ and $0.002 \mu \mathrm{g} / \mathrm{L}$ for $\mathrm{Cr}(\mathrm{III})$ and $\mathrm{Cr}(\mathrm{VI})$ respectively. The LOQ were calculated as ten times the background equivalent concentration (BEC); these were $0.067 \mu \mathrm{g} / \mathrm{L}$ and $0.008 \mu \mathrm{g} / \mathrm{L}$ for $\mathrm{Cr}(\mathrm{III})$ and $\mathrm{Cr}(\mathrm{VI})$ respectively. The results of the three in-house spiked EBC samples shown in Table 2 gave an average spiked recovery of $105 \%$ for the EBC sample spiked only with $2 \mu \mathrm{g} / \mathrm{L} \mathrm{Cr}(\mathrm{III}) ; 98 \%$ for the EBC sample spiked only with $2 \mu \mathrm{g} / \mathrm{L} \mathrm{Cr}(\mathrm{VI})$, along with $99 \%$ and $95 \%$ for the EBC sample spiked with both $2 \mu \mathrm{g} / \mathrm{L} \mathrm{Cr}(\mathrm{III})$ and $\mathrm{Cr}(\mathrm{VI})$ respectively. In each analysis the PTM for $\mathrm{Cr}(\mathrm{VI})$ in water gave consistently accurate results which were all within the acceptance range. As shown in Table 2, no conversion of chromium species was observed during analysis with the $\mathrm{Cr}(\mathrm{III})$ concentrations less than the LOQ when measuring the PTM and EBC in-house sample spiked only with $2 \mu \mathrm{g} / \mathrm{L} \mathrm{Cr}(\mathrm{VI})$ (no $\mathrm{Cr}(\mathrm{III})$ should be present). The $\mathrm{Cr}(\mathrm{VI})$ concentration was also less than the LOQ when measuring the EBC in-house sample spiked only with $2 \mu \mathrm{g} / \mathrm{L} \mathrm{Cr}(\mathrm{III})$ (no $\mathrm{Cr}(\mathrm{VI})$ present).

\subsubsection{Urine samples - total chromium}

The determination of total chromium in urine is an established UKAS accredited method at the Health and Safety Laboratory. The LOD is $1 \mathrm{nmol} / \mathrm{L}(0.05 \mu \mathrm{g} / \mathrm{L})$ (uncorrected for creatinine). Both urine CRMs, Biorad Level 1 and Clinchek level 2 are certified for total chromium in urine; all gave good results within the target range, this is shown in Table 2.

\subsection{Study results}

Of the 22 volunteers (16 males, 6 females) in the control group only 1 (male) was a smoker, the remaining 21 volunteers were non-smokers. Of the 58 volunteers (53 males, 5 females) in the occupationally exposed worker group, 33 were non-smokers ( 3 female, 29 male) and 25 were smokers ( 1 female, 24 male). Of these 58 workers, 35 were classified as $\mathrm{Cr}(\mathrm{VI})$ Workers, 12 as $\mathrm{Non}-\mathrm{Cr}(\mathrm{VI})$ Workers and 11 as Other Workers. Of the $35 \mathrm{Cr}(\mathrm{VI})$ Workers only 1 volunteer was female, similarly of the 12 Non-Cr(VI) Workers only 1 volunteer was female. Of the 11 Other Workers 3 volunteers were female and 8 were male.

The EBC results for controls and workers are shown in Fig. 2 and presented in Table 3. The results for the EBC analysis from the control group showed that $84 \%$ and $91 \%$ were below the LOQ for $\mathrm{Cr}(\mathrm{III})$ and $\mathrm{Cr}(\mathrm{VI})$ respectively, compared with only $5 \%$ and $3 \%$ for the occupationally exposed workers.

Fig. 2 and Table 3 show that the median values for $\mathrm{Cr}(\mathrm{III})$ in EBC from the control group are below the LOQ. Furthermore all $\mathrm{Cr}(\mathrm{III})$ found in $\mathrm{EBC}$ results from a Non- $\mathrm{Cr}(\mathrm{VI})$ worker or Other Worker were above the LOQ. The median concentration of $\mathrm{Cr}(\mathrm{III})$ in EBC samples for both pre and post working week of all 58 occupationally exposed workers is very similar, with a median value of $0.59 \mu \mathrm{g} / \mathrm{L}$ for pre working week and $0.54 \mu \mathrm{g} / \mathrm{L}$ for post working week. The $\mathrm{Cr}(\mathrm{VI})$ Workers also showed similar median concentrations of $\mathrm{Cr}(\mathrm{III})$ in $\mathrm{EBC}$ of $0.58 \mu \mathrm{g} / \mathrm{L}$ and $0.51 \mu \mathrm{g} / \mathrm{L}$ for pre and post working week samples respectively. The Other Workers had the highest median concentrations of $\mathrm{Cr}(\mathrm{III})$ in EBC of 0.70 and $0.66 \mu \mathrm{g} / \mathrm{L}$ for pre and post working week samples. The Non- $\mathrm{Cr}(\mathrm{VI})$ Workers gave the lowest median concentrations of pre and post working week samples for $\mathrm{Cr}(\mathrm{III})$ in $\mathrm{EBC}$ of 0.38 and $0.54 \mu \mathrm{g} / \mathrm{L}$ respectively. The maximum $\mathrm{Cr}(\mathrm{III})$ concentration measured was $11.02 \mu \mathrm{g} / \mathrm{L}$ and was from a post working week EBC sample of a $\mathrm{Cr}(\mathrm{VI})$ Worker, compared to the maximum $\mathrm{Cr}(\mathrm{III})$ in $\mathrm{EBC}$ from a Non- $\mathrm{Cr}(\mathrm{VI})$ Worker and an Other Worker both resulting from a pre working week sample, of $9.1 \mu \mathrm{g} / \mathrm{L}$ and $2.97 \mu \mathrm{g} / \mathrm{L}$ respectively.

Fig. 2 and Table 3 show that the median values for $\mathrm{Cr}(\mathrm{VI})$ in $\mathrm{EBC}$ from the control group are below the LOQ. As with $\mathrm{Cr}(\mathrm{III})$, there were no $\mathrm{Cr}(\mathrm{VI})$ in $\mathrm{EBC}$ results from a Non- $\mathrm{Cr}(\mathrm{VI})$ worker or Other Worker less than the LOQ. The results of $\mathrm{Cr}(\mathrm{VI})$ in EBC samples for all 58 occupationally exposed workers showed a median concentration increase from 0.58 to $0.72 \mu \mathrm{g} / \mathrm{L}$ of pre to post working week samples. An increase in $\mathrm{Cr}(\mathrm{VI}) \mathrm{EBC}$ levels was observed between the pre and post working samples for both median $(0.72-0.91 \mu \mathrm{g} / \mathrm{L})$ and 90th percentile concentrations $(2.81-4.56 \mu \mathrm{g} / \mathrm{L})$ for the $\operatorname{Cr}(\mathrm{VI})$ Workers. This trend was also observed in the Non-Cr(VI) Workers to a lesser extent with a pre working week median concentration of $0.29 \mu \mathrm{g} / \mathrm{L}$ increasing to a post working week median concentration 


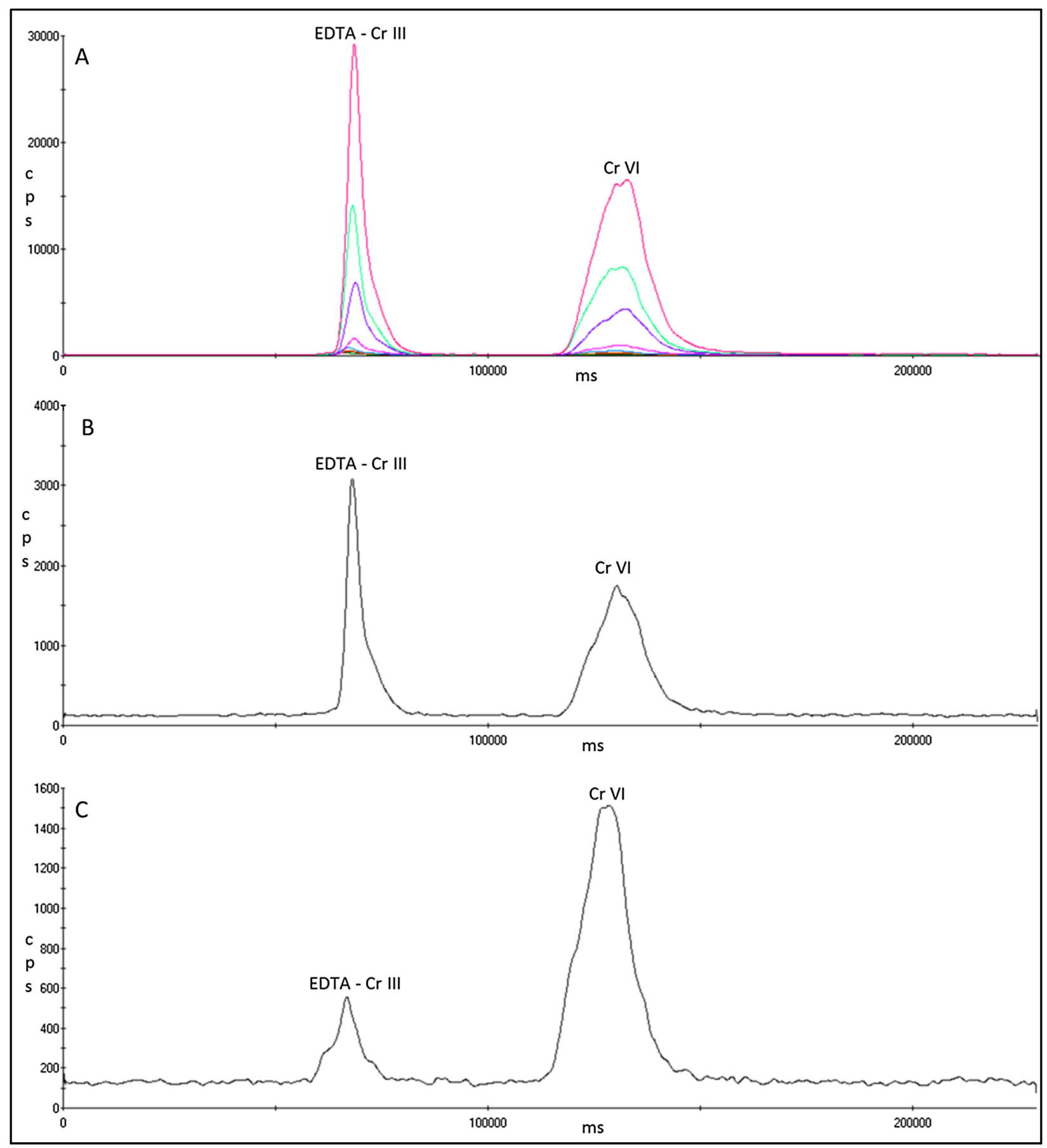

Fig. 1. Chromatograms showing full separation of EDTA-Cr(III) and $\mathrm{Cr}(\mathrm{VI})$ using an ESI OneFAST system coupled to a Dionex AG7 anion exchange column and ICP-MS using a nitric acid and ammonia mixed mobile phase. (A) Overlay of the individual standard calibration chromatograms of $0-2 \mu \mathrm{g} / \mathrm{L}$ calibration. (B) An in-house spiked EBC sample $2 \mu \mathrm{g} / \mathrm{L}$ mixed $\mathrm{Cr}(\mathrm{III})$ and $\mathrm{Cr}(\mathrm{VI})$. (C) An EBC sample from an occupationally exposed volunteer.

of $0.44 \mu \mathrm{g} / \mathrm{L}$. A two-fold increase from $0.31 \mu \mathrm{g} / \mathrm{L}$ (pre working week) to $0.62 \mu \mathrm{g} / \mathrm{L}$ (post working week) was observed in the median EBC of the Other Workers. The Other Workers showed a higher median and 90th percentile for both the pre and post working week $\mathrm{Cr}(\mathrm{VI})$ in EBC samples than the Non-Cr(VI) Workers, but not the $\mathrm{Cr}(\mathrm{VI})$ Workers. The maximum $\mathrm{Cr}(\mathrm{VI})$ concentration in $\mathrm{EBC}$ of $27.3 \mu \mathrm{g} / \mathrm{L}$ was from the same $\mathrm{Cr}(\mathrm{VI})$ Worker's post working week EBC sample that contained the highest $\mathrm{Cr}(\mathrm{III})$ concentration in $\mathrm{EBC}$. Similarly to $\mathrm{Cr}(\mathrm{III})$ in $\mathrm{EBC}$ the highest level of $\mathrm{Cr}(\mathrm{VI})$ in both $\mathrm{Non}-\mathrm{Cr}(\mathrm{VI})$ Workers and Other Workers was from a pre working week sample, of 8.31 and $2.59 \mu \mathrm{g} / \mathrm{L}$ respectively. 
Table 2

Results obtained for the two species of chromium in the in house spiked EBC samples and the PTM samples and the urinary chromium CRM.

\begin{tabular}{|c|c|c|c|c|}
\hline & $\begin{array}{l}\text { In-house spiked EBC }(2 \mu \mathrm{g} / \mathrm{L}) \\
\operatorname{Cr}(\mathrm{III})(\mathrm{n}=58)(\% \text { recovery })\end{array}$ & $\begin{array}{l}\text { In-house spiked EBC }(2 \mu \mathrm{g} / \mathrm{L}) \\
\mathrm{Cr}(\mathrm{VI})(\mathrm{n}=56)(\% \text { recovery })\end{array}$ & $\begin{array}{l}\text { In-house spiked EBC }(2 \mu \mathrm{g} / \mathrm{L}) \mathrm{Cr}(\mathrm{III}) \\
\& \mathrm{Cr}(\mathrm{VI}) \operatorname{mix}(\mathrm{n}=58)(\% \text { recovery })\end{array}$ & $\begin{array}{l}\text { PTM LRAA1427 }(\mathrm{n}=57) \\
\text { Target range } 2.28-3.28\end{array}$ \\
\hline $\mathrm{Cr}(\mathrm{III}) \mathrm{EBC}$ & $2.09 \pm 0.3(105 \%)$ & $<\mathrm{LOQ}$ & $1.98 \pm 0.26(99 \%)$ & $<\mathrm{LOQ}$ \\
\hline \multirow[t]{3}{*}{$\mathrm{Cr}(\mathrm{VI}) \mathrm{EBC}$} & $<$ LOQ & $1.95 \pm 0.16(98 \%)$ & $1.90 \pm 0.26(95 \%)$ & $2.63 \pm 0.17$ \\
\hline & Biorad Level 1-69161 $(n=14)$ & Biorad Level 1-69171 $(n=35)$ & Clinchek Level 2-122 ( $\mathrm{n}=18)$ & Clinchek Level 2-432 $(n=31)$ \\
\hline & Target range $0.359-0.995 \mu \mathrm{g} / \mathrm{L}$ & Target range $0.575-2.53 \mu \mathrm{g} / \mathrm{L}$ & Target range $16.2-24.4 \mu \mathrm{g} / \mathrm{L}$ & Target range $15.9-23.8 \mu \mathrm{g} / \mathrm{L}$ \\
\hline $\begin{array}{l}\text { Urinary total } \\
\text { chromium }\end{array}$ & $0.82 \pm 0.09$ & $0.90 \pm 0.16$ & $18.9 \pm 1.20$ & $18.6 \pm 0.75$ \\
\hline
\end{tabular}

All of the urinary total chromium results for the occupationally exposed workers were above the limit of quantification. As shown in Fig. 3 all the urine samples from the control group were below the HSL background unexposed reference range of $<2.9 \mu \mathrm{mol} / \mathrm{mol}$ creatinine.

Analysis of the urine samples from the occupationally exposed workers showed; that 53\% were below the background unexposed reference range, $31 \%$ were above the HSL UK background unexposed reference range but below the BMGV of $10 \mu \mathrm{mol} / \mathrm{mol}$ creatinine whilst $16 \%$ exceeded the BMGV. As shown in Table 3 , the median values for all 58 workers of the occupationally exposed group showed a moderate increase in total chromium in urine from 1.9 to $3.3 \mu \mathrm{mol} / \mathrm{mol}$ creatinine for pre to post working week samples. However, the 90th percentile values showed over a 4fold increase from 3.1 to $13.7 \mu \mathrm{mol} / \mathrm{mol}$ creatinine for pre to post working week samples. The increase of the median and 90th percentile from the pre working week samples to the post working samples was also observed for all three categories of worker, with a significant increase observed in the 90th percentile for both $\mathrm{Cr}(\mathrm{VI})$ Workers and Non-Cr(VI) Workers from pre to post working week samples. The highest urinary total chromium value was found in a post working week sample of a Non-Cr(VI) Worker, being $37.1 \mu \mathrm{mol} / \mathrm{mol}$ creatinine, compared to maximum value of $6.0 \mu \mathrm{mol} / \mathrm{mol}$ creatinine for an Other Worker. The highest urinary total chromium value for both pre and post working week from a $\mathrm{Cr}(\mathrm{VI})$ worker was 29.4 and $34.1 \mu \mathrm{mol} / \mathrm{mol}$ creatinine respectively and came from the same volunteer. However this volunteer was not responsible for the highest $\mathrm{Cr}(\mathrm{III})$ and $\mathrm{Cr}(\mathrm{VI})$ levels found in $\mathrm{EBC}$.

\subsection{Statistical results}

General statistical analysis was performed using Prism Graphpad Version 6 for Windows. Due to the skewness and distribution from normality of the data, all the data was log transformed. The log of the data is normally distributed enabling parametric statistical analysis. Results below the LOQ were replaced with half the LOQ. Paired $t$-tests showed there was a significant difference for total chromium levels in urine between pre and post working week samples for all the occupationally exposed workers $(p=0.03)$. However there was no significant difference between pre and post working week EBC samples for $\mathrm{Cr}(\mathrm{III})(\mathrm{p}=0.63)$ or $\mathrm{Cr}(\mathrm{VI})(\mathrm{p}=0.13)$. Unpaired $t$-tests found there was a strong significant difference between workers and controls for total chromium in urine $(p<0.0001)$, as well as $\mathrm{Cr}(\mathrm{III})$ in EBC samples $(\mathrm{p}<0.0001)$ and $\mathrm{Cr}(\mathrm{VI})$ in EBC samples $(\mathrm{p}<0.0001)$. As demonstrated in Fig. 4, no correlation was found when comparing post working week levels of total chromium in urine with $\mathrm{Cr}(\mathrm{III})$ in $\mathrm{EBC}\left(\mathrm{r}^{2}=0.02, \mathrm{p}=0.35\right)$, or post working week levels of total chromium in urine samples with the sum of $\mathrm{Cr}(\mathrm{III})$ and $\mathrm{Cr}(\mathrm{VI})$ in EBC samples $\left(\mathrm{r}^{2}=0.04, \mathrm{p}=0.12\right)$. However, a weak moderate correlation was observed when comparing post working week levels of total chromium in urine with $\mathrm{Cr}(\mathrm{VI})$ in $\mathrm{EBC}$ samples $\left(\mathrm{r}^{2}=0.11, \mathrm{p}=0.01\right)$

Statistical analysis was not performed for gender differences because of the small proportion (9\%) of females forming the occupationally exposed group in this study. There was also no statistical significant difference observed between smokers and non-smokers or between workers who wore facemasks/respiratory protective equipment (RPE) and those who did not.

\section{Discussion}

Reported here are the determined chromium speciation results of $\mathrm{Cr}(\mathrm{III})$ and $\mathrm{Cr}(\mathrm{VI})$ concentrations in $\mathrm{EBC}$ and total chromium concentrations in urine from pre and post working week samples collected from 58 occupationally exposed workers and 22 controls not occupationally exposed to chromium.

The LOD and LOQ reported in this novel chromium speciation study are very low, thus making this a suitable method for the

Table 3

Statistical summary of the mean, median and 90th percentile concentrations of pre working week (Pre W-Wk) and post working week (Post W-Wk) samples for Cr(III) and $\mathrm{Cr}(\mathrm{VI})$ in $\mathrm{EBC}$ in $\mu \mathrm{g} / \mathrm{L}$ and total chromium in urine in $\mu \mathrm{mol} / \mathrm{mol}$ creatinine for both controls and workers.

\begin{tabular}{|c|c|c|c|c|c|c|c|}
\hline & & \multicolumn{2}{|c|}{$\mathrm{Cr}(\mathrm{III})$ in $\mathrm{EBC} \mu \mathrm{g} / \mathrm{L}$} & \multicolumn{2}{|c|}{$\mathrm{Cr}(\mathrm{VI})$ in $\mathrm{EBC} \mu \mathrm{g} / \mathrm{L}$} & \multicolumn{2}{|c|}{ Total $\mathrm{Cr}$ in Urine $\mu \mathrm{mol} / \mathrm{mol}$ creatinine } \\
\hline & & Pre $W-W k$ & Post W-Wk & Pre W-Wk & Post W-Wk & Pre W-Wk & Post W-Wk \\
\hline \multirow{3}{*}{$\begin{array}{l}\text { All Workers: } \\
\mathrm{n}=58\end{array}$} & Median & 0.59 & 0.54 & 0.58 & 0.72 & 1.9 & 3.3 \\
\hline & P90 & 2.43 & 4.21 & 2.01 & 2.45 & 3.1 & 13.7 \\
\hline & Range & $0.19-9.1$ & $0.14-11.03$ & $0.04-9.69$ & $0.01-27.3$ & $0.5-29.4$ & $0.6-37.1$ \\
\hline $\mathrm{Cr}(\mathrm{VI})$ Workers: & Median & 0.58 & 0.51 & 0.72 & 0.91 & 3.4 & 4.7 \\
\hline \multirow{2}{*}{$\mathrm{n}=35$} & P90 & 2.15 & 2.98 & 2.81 & 4.56 & 14.8 & 20.4 \\
\hline & Range & $<$ LOQ-3.38 & $<$ LOQ-11.02 & $<$ LOQ-9.69 & $<$ LOQ-27.3 & $0.6-29.4$ & $1.1-34.1$ \\
\hline Non $\mathrm{Cr}(\mathrm{VI})$ & Median & 0.38 & 0.54 & 0.29 & 0.44 & 1.5 & 2.1 \\
\hline Workers & P90 & 0.86 & 1.41 & 0.76 & 1.71 & 3.7 & 23.7 \\
\hline $\mathrm{n}=12$ & Range & $0.19-9.1$ & $0.20-4.56$ & $0.04-8.31$ & $0.06-2.45$ & $0.6-8.6$ & $0.6-37.1$ \\
\hline Other Workers & Median & 0.70 & 0.66 & 0.31 & 0.62 & 1.2 & 1.5 \\
\hline \multirow[t]{2}{*}{$\mathrm{n}=11$} & P90 & 2.2 & 2.05 & 2.01 & 1.84 & 1.6 & 2.7 \\
\hline & Range & $0.27-2.97$ & $0.14-2.21$ & $0.03-2.59$ & $0.01-2.20$ & $0.5-5.1$ & $0.8-6.0$ \\
\hline \multirow{3}{*}{$\begin{array}{l}\text { Controls } \\
n=22\end{array}$} & Median & $<\mathrm{LOQ}$ & $<\mathrm{LOQ}$ & $<\mathrm{LOQ}$ & $<\mathrm{LOQ}$ & 0.4 & 0.4 \\
\hline & P90 & 0.09 & 0.09 & 0.01 & $<\mathrm{LOQ}$ & 0.6 & 1.1 \\
\hline & Range & $<$ LOQ-0.27 & $<$ LOQ-4.97 & $<$ LOQ-0.07 & $<$ LOQ-0.12 & $<$ LOQ-1.3 & $<$ LOQ-1.4 \\
\hline
\end{tabular}



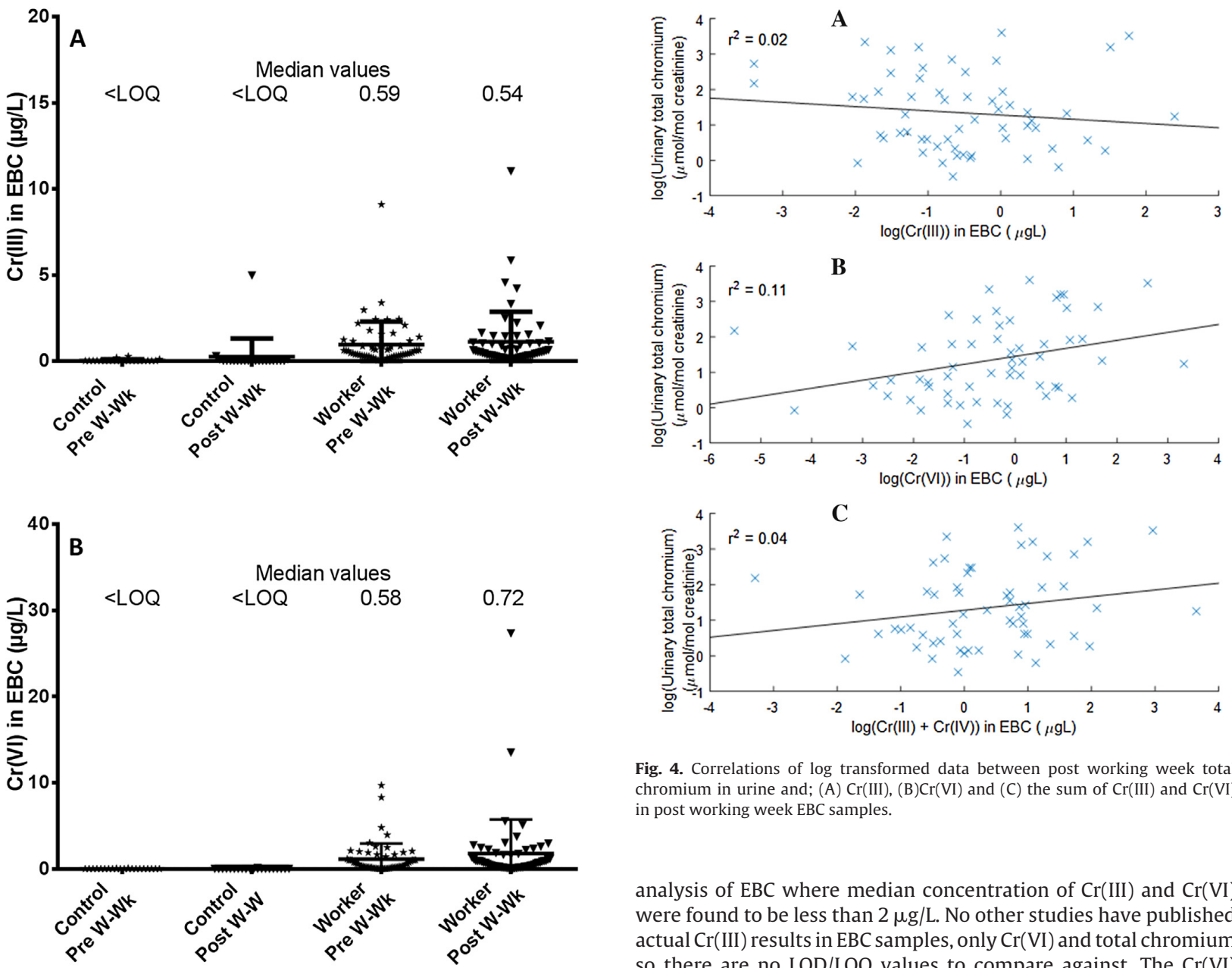

Fig. 4. Correlations of log transformed data between post working week tota chromium in urine and; (A) $\mathrm{Cr}(\mathrm{III}),(\mathrm{B}) \mathrm{Cr}(\mathrm{VI})$ and $(\mathrm{C})$ the sum of $\mathrm{Cr}(\mathrm{III})$ and $\mathrm{Cr}(\mathrm{VI})$ in post working week EBC samples.

Fig. 2. Scatter plots with median concentrations $(\mu \mathrm{g} / \mathrm{L})$ from pre working week (Pre W-Wk) to post working week (Post W-Wk) samples for (A) Cr(III) in EBC and (B) $\mathrm{Cr}(\mathrm{VI})$ in EBC for both occupationally exposed volunteers and the unexposed control group.

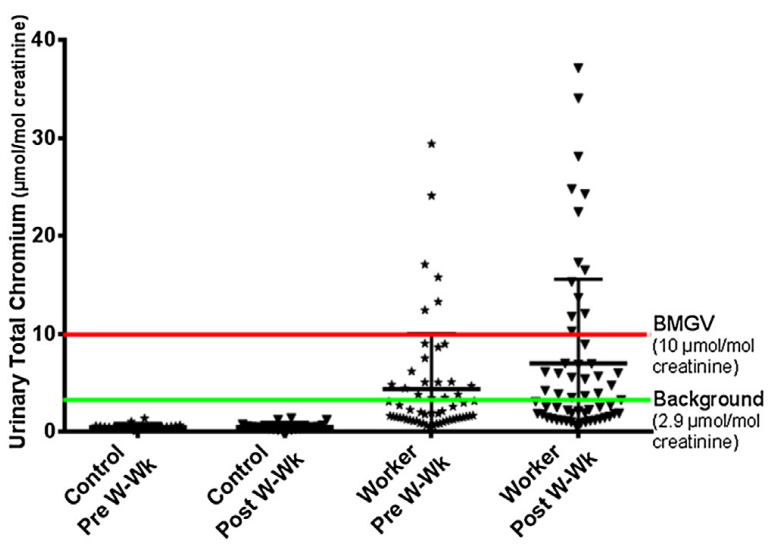

Fig. 3. Scatter plot showing the results of total chromium in urine $(\mu \mathrm{mol} / \mathrm{mol}$ creatinine) for both the occupationally exposed workers and the control group for both pre working week (Pre W-Wk) and post working week (Post W-Wk) samples. Showing results in comparison to the background unexposed reference range and the BMGV.

analysis of EBC where median concentration of $\mathrm{Cr}(\mathrm{III})$ and $\mathrm{Cr}(\mathrm{VI})$ were found to be less than $2 \mu \mathrm{g} / \mathrm{L}$. No other studies have published actual $\mathrm{Cr}(\mathrm{III})$ results in $\mathrm{EBC}$ samples, only $\mathrm{Cr}(\mathrm{VI})$ and total chromium so there are no LOD/LOQ values to compare against. $\mathrm{The} \mathrm{Cr}(\mathrm{VI})$ LOD and LOQ reported here is lower than other reported studies (Goldoni et al., 2010; Goldoni et al., 2006). However, the LOD and LOQ reported in this study are achieved using ICP-MS whilst the LOD of $0.2 \mu \mathrm{g} / \mathrm{L}$ reported by Goldoni et al. $(2010,2006)$ uses electrothermal atomic absorption spectrometry (ETAAS). Other publications reporting chromium in EBC were for total chromium only and the LODs reported were a limiting factor in their results (Gube et al., 2010; Hoffmeyer et al., 2011).

The background unexposed reference range established by the Health and Safety Laboratory, for total chromium in urine is $<2.9 \mu \mathrm{mol} / \mathrm{mol}$ creatinine (Morton et al., 2014). For the urinary total chromium results for exposed workers, $47 \%$ exceeded this value, indicating that nearly half of the urine samples provided by the 58 workers showed some occupational exposure to $\mathrm{Cr}(\mathrm{VI}) \mathrm{com}$ pounds. Furthermore, $16 \%$ were above the BMGV of $10 \mu \mathrm{mol} / \mathrm{mol}$ creatinine, indicating that 19 urine samples provided by the 58 workers showed significant exposure to $\mathrm{Cr}(\mathrm{VI})$ compounds. This exposure demonstrates that the occupationally exposed workers who took part in this study were a suitable cohort to successfully meet the objective of this study and determine if $\mathrm{Cr}(\mathrm{III})$ and $\mathrm{Cr}(\mathrm{VI})$ could be detected and measured in real EBC samples. As shown in Table 3, the 90th percentile of urinary total chromium for both Other Workers and the control group are within the background unexposed reference range, whereas the $\mathrm{Cr}(\mathrm{VI})$ Workers and Non- $\mathrm{Cr}(\mathrm{VI})$ Workers is twice the BMGV of $10 \mu \mathrm{mol} / \mathrm{mol}$ creatinine, indicating a strong association with chromium occupational exposure and urinary chromium measurements. 
It is important to note that the study outlined here was a pilot feasibility study to ascertain if both $\mathrm{Cr}(\mathrm{III})$ and $\mathrm{Cr}(\mathrm{VI})$ could be determined in EBC samples from workers occupationally exposed to $\mathrm{Cr}(\mathrm{VI})$ compounds by inhalation. Consequently, no environmental monitoring such as air monitoring, surface wipes or hand wipe sampling was performed, nor was information taken regarding length of time since last potential exposure or length of time and levels of potential exposure from any of the occupationally exposed workers prior to providing a EBC or urine sample.

The statistical analysis found a lack of correlation between levels of total chromium in urine with $\mathrm{Cr}$ (III) in EBC and levels of total chromium in urine with the sum of $\mathrm{Cr}(\mathrm{VI})$ and $\mathrm{Cr}(\mathrm{III})$ in EBC, however a low moderate correlation was found between levels of total chromium in urine with $\mathrm{Cr}(\mathrm{VI})$ in EBC. This lack of correlation is in agreement with other studies where no correlation was found between total chromium in urine with $\mathrm{Cr}(\mathrm{VI})$ in EBC (Goldoni et al., 2006) or with total chromium in urine compared with total chromium in EBC (Caglieri et al., 2006; Goldoni et al., 2010). However, this may be expected considering urinary chromium levels will reflect all three routes of exposure (inhalation, dermal and ingestion); whereas EBC samples will reflect mainly inhalation exposure with some contribution from what is present in the mouth but not exposure by dermal absorption or ingestion. Interestingly, Goldoni et al. (2010) found there was a significant correlation between total chromium in post shift EBC samples and RBC samples only. This is not always the case, as better correlation of urine with EBC samples has been observed for other elements (Goldoni et al., 2004).

Whilst there is a lack of statistical significance between the pre and post working week samples for both $\mathrm{Cr}(\mathrm{III})$ and $\mathrm{Cr}(\mathrm{VI})$ in $\mathrm{EBC}$, all the levels were higher than the controls. The data showed that $48 \%$ of the occupationally exposed group showed an increase of the concentrations of $\mathrm{Cr}(\mathrm{III})$ in EBC from the pre to post work week sample, and $59 \%$ showed an increase of the concentrations of $\mathrm{Cr}(\mathrm{VI})$ in $\mathrm{EBC}$ from the pre to post working week sample. However, chromium in lungs has shown to accumulate upon repeat inhalation (Hoet, 2005), something that is likely to happen in an occupational setting. In addition, reduction of $\mathrm{Cr}(\mathrm{VI})$ to $\mathrm{Cr}(\mathrm{III})$ by glutathione was found to be much slower than ascorbate leading to a longer residence of chromium in lungs (Petersen et al., 2000), meaning the higher levels of chromium in pre working week EBC samples may be the result of high exposure the previous week. It must also be noted that although both urine and EBC samples collected on the Monday morning were considered pre working week, the workers had already entered the work place. Although workers had been instructed not to start official work that could result in inhalation exposure (e.g. grinding or electroplating), they had started pre work preparation, meaning some exposure may have already occurred from chromium levels in the air of the workplace or from preparation procedures. This lack of correlation may also be related to the combination of a high proportion of low $\mu \mathrm{g} / \mathrm{L}$ values for a sample size of only 58 occupationally exposed workers. In addition, the lack of information regarding exposure of the workers prior to providing the post working week EBC sample will be a contributing factor. No statistically significant difference was found between smokers and non-smokers for $\mathrm{Cr}(\mathrm{III})$ or $\mathrm{Cr}(\mathrm{VI})$ in $\mathrm{EBC}$ or total chromium in urine. Nor was there a statistical significant difference found between those individuals that wore any type of facemask/respiratory protective equipment (RPE) and those that did not. However, this outcome is not unexpected when tandem environmental analysis has not been performed and little information is known on length of time since last exposure or length of time the worker has experienced $\mathrm{Cr}(\mathrm{VI})$ exposure or the levels of exposure. This uncertainty, along with the fact these comparisons would reduce the dataset substantially, make it difficult to compare these more specific data groups and extrapolate any type of significance that would be statistically robust to investigate as to whether wearing RPE or smoker status affected observed levels of $\mathrm{Cr}$ (III) or $\mathrm{Cr}(\mathrm{VI})$ in EBC samples.

A similar study by Goldoni et al. (2006), determined $\mathrm{Cr}(\mathrm{VI})$ in EBC samples from 10 chromium electroplaters; they looked at overnight chromium decrease with EBC samples taken post shift and again the following morning pre shift ( $15 \mathrm{~h}$ since exposure). A decrease in $\mathrm{Cr}(\mathrm{VI})$ levels from overnight post to pre shift was observed, with concentrations of $\mathrm{Cr}(\mathrm{VI})$ found in $\mathrm{EBC}$ samples ranging from $0.1-2.9 \mu \mathrm{g} / \mathrm{L}$ for the post shift EBC samples and $0.1-2.1 \mu \mathrm{g} / \mathrm{L}$ for the pre shift EBC samples. However only 10 workers were sampled and seven of the 20 EBC samples collected were found to be below the LOD. The concentrations of $\operatorname{Cr}(\mathrm{VI})$ in $\mathrm{EBC}$ reported here in the current study were higher, with overall $(n=58)$ concentrations of $\mathrm{Cr}(\mathrm{VI})$ in workers ranging from $0.04-9.69 \mu \mathrm{g} / \mathrm{L}$ for the pre shift EBC samples and 0.01-27.35 $\mu \mathrm{g} / \mathrm{L}$ for the post shift EBC samples. A later study by Goldoni et al. (2010) reported a similar study method but with tandem urine, red blood cell and plasma sampling alongside the EBC sample collection. The median values reported in this later study compare well with the results reported here in this study. For example, median total chromium in urine levels increased from 4 to $6 \mu \mathrm{mol} / \mathrm{mol}$ creatinine in Goldoni's study compared to a median increase of $1.9-3.3 \mu \mathrm{mol} / \mathrm{mol}$ creatinine for all workers and $3.4-4.7 \mu \mathrm{mol} / \mathrm{mol}$ creatinine for $\mathrm{Cr}(\mathrm{VI})$ Workers reported here in this study. Additionally, in Goldoni's study median values for $\mathrm{Cr}(\mathrm{VI})$ in EBC samples increased from 0.3 to $1.0 \mu \mathrm{g} / \mathrm{L} \mathrm{com}$ pared to median values increasing from 0.58 to $0.72 \mu \mathrm{g} / \mathrm{L}$ for all workers and $0.72-0.91 \mu \mathrm{g} / \mathrm{L}$ for $\mathrm{Cr}(\mathrm{VI})$ Workers reported here in this study. The studies by Goldoni et al. (2006, 2010) in addition to the work reported here indicate the need for a comprehensive EBC collection protocol to better understand dwell, half-life and excretion rates of chromium in EBC.

Also of interest is the two fold increase of $0.31 \mu \mathrm{g} / \mathrm{L}$ (pre working week) to $0.62 \mu \mathrm{g} / \mathrm{L}$ (post working week) median levels of $\mathrm{Cr}(\mathrm{VI})$ in EBC for the Other Workers. Although the median values are not higher than the $\mathrm{Cr}(\mathrm{VI})$ Workers, they are higher than the median values of the Non-Cr(VI) Workers, indicating inhalation exposure is not limited solely to workers operating directly in areas of the workplace where chromium processes occur. Chromium exposure of Other Workers is more evident when the median values for both $\mathrm{Cr}(\mathrm{III})$ and $\mathrm{Cr}(\mathrm{VI})$ in EBC are compared against the control group values, which were all below the LOQ, however there were only 11 volunteers in the Other Workers group. No other studies have determined chromium EBC measurements in this type of worker cohort for comparison.

In recent literature, there is reference to the need for a marker in EBC samples to standardise for dilution (Kuban and Foret, 2013; Rosias, 2012), in the same way creatinine is utilised to correct for concentration of urine. It is suggested that the droplets of fluid from the respiratory tract can be diluted by the water vapour found in EBC and will vary amongst individuals (Rosias, 2012). At present, there is no consensus on a suitable and accurate biomarker to correct for dilution, as smoking status, inflammatory conditions such as asthma or the inhaled contaminant itself have affected their usefulness in biomarkers studies (Dodig and Cepelak, 2013; Hoffmeyer et al., 2015). However Rosias (2012) and Reinhold and Knobloch (2010) have suggested EBC results be expressed per volume of EBC collected. Unfortunately, in this study neither approach was explored. However, it is something that must be taken into consideration in future EBC studies.

To enable the future of EBC as a biomarker for occupational exposure to chromium species there needs to be an understanding of the dwell time of chromium species detectable in an EBC sample after exposure has ceased. In addition to a better understanding of the relationship between levels of exposure and EBC measure- 
ments is essential. The latter could be achieved with correlation to air measurements.

Previously known CRMs for either $\mathrm{Cr}(\mathrm{III})$ and/or $\mathrm{Cr}(\mathrm{VI})$ by NIST and IRMM have either been discontinued or withdrawn. For the future success of chromium speciation in EBC it is important that CRMs in either water or EBC are made available. In addition, the future of EBC as a biological matrix will rely on CRMs for other trace elements.

\section{Conclusion}

This is the first study to report the detection and measurement of $\mathrm{Cr}(\mathrm{III})$ and $\mathrm{Cr}(\mathrm{VI})$ in 'real' exhaled breath condensate (EBC) samples of volunteers. The analytical method outlined in this feasibility study has shown that both $\mathrm{Cr}(\mathrm{III})$ and $\mathrm{Cr}(\mathrm{VI})$ can be simultaneously detected and measured in EBC samples from a cohort of volunteers who are occupationally exposed to $\mathrm{Cr}(\mathrm{VI})$ compounds by inhalation. The occupationally exposed workers showed significantly higher levels of both $\mathrm{Cr}(\mathrm{III})$ and $\mathrm{Cr}(\mathrm{VI})$ than in the control group. However no statistically significant difference was found between pre and post working week samples for $\mathrm{Cr}(\mathrm{III})$ and $\mathrm{Cr}(\mathrm{VI})$ in $\mathrm{EBC}$ of the occupationally exposed workers. The speciation method demonstrates high sensitivity with low limits of detection and quantification. The next stage of this study will be to undertake site visits to perform air monitoring in tandem with EBC sample collection to further understand and correlate the relationship between levels of exposure and $\mathrm{EBC}$ results for chromium.

\section{Funding}

This publication and the work it describes were funded by the Health \& Safety Executive (HSE). Its contents, including any opinions and/or conclusions expressed, are those of the authors alone and do not necessarily reflect HSE policy.

\section{Acknowledgements}

The authors would like to thank all the volunteers for participating in this study, along with independent metal finishing consultants John Torr and John Williams for all their knowledge and help during this project.

\section{References}

Agency for Toxic Substances \& Disease Registry, 2012. Toxicological Profile for Chromium. http://www.atsdr.cdc.gov/TOXPROFILES/TP.ASP?ID=62\&TID=17/ (Accessed 11 April 2016).

Broding, H.C., Michalke, B., Goen, T., Drexler, H., 2009. Comparison between exhaled breath condensate analysis as a marker for cobalt and tungsten exposure and biomonitoring in workers of a hard metal alloy processing plant Int. Arch. Occup. Environ. Health 82 (5), 565-573.

Caglieri, A., Goldoni, M., Acampo, O., Andreoli, R., Vettori, M.V., Corradi, M., Apostoli, P., Mutti, A., 2006. The effect of inhaled chromium on different exhaled breath condensate biomarkers among chrome-plating workers. Environ. Health Perspect. 114 (4), 542-546.

Cocker, J., Mason, H.J., Warren, N.D., Cotton, R.J., 2011. Creatinine adjustment of biological monitoring results. Occup. Med. 61 (5), 349-353.

Corradi, M., Mutti, A., 2005. Exhaled breath analysis: from occupational to respiratory medicine. Acta Biomed. 76, 20-29.

De Flora, S., Serra, D., Camoirano, A., Zanacchi, P., 1989. Metabolic reduction of chromium as related to its carcinogenic properties. Biol. Trace Elem. Res. 21, 179-187.

De Flora, S., 2000. Threshold mechanisms and site specificity in chromium(VI) carcinogenesis. Carcinogenesis 21 (4), 533-541.

Dodig, S., Cepelak, I., 2013. Exhaled breath condensate - from an analytical point of view. Biochem. Med. 23 (3), 281-295

Felix, P.M., Almeida, S.M., Franco, C., Almeida, A.B., Lopes, C., Claro, M.I., fragosos, E. Teles, C., Wolterbeek, H.T., Pinheiro, T., 2015. The suitability of EBC-Pb as a new biomarker to assess occupational exposure to lead. Int. J. Environ. Health Res. $25(1), 67-80$.
Genovese, G., Castglia, L., Pieri, M., Novi, C., D’Angelo, R., Sannolo, N., Lamberti, M., Miraglia, N., 2015. J. Occup. Environ. Hyg. 12 (8), 518-524,

Goldoni, M., Catalani, S., De Palma, G., Manini, P., Acampo, O., Corradi, M., Bergonzi, R., Apostoli, P., Mutti, A., 2004. Exhaled breath condensate as a suitable matrix to assess lung dose and effects in workers exposed to cobalt and tungsten. Environ. Health Perspect. 112 (13), 1293-1298.

Goldoni, M., Caglieri, A., Poli, D. Vettori, M. V., Corradi, M., Apostoli, P., Mutti, A. 2006. Determination of hexavalent chromium in exhaled breath condensate
20., and environmental air among chrome plating workers. Anal. Chim. Acta 562 (2), 229-235.

Goldoni, M., Caglieri, A., De Palma, G., Acampo, O., Gergelova, P., Corradi, M., Apostoli, P., Mutti, A., 2010. Chromium in exhaled breath condensate (EBC), erythrocytes, plasma and urine in the biomonitoring of chrome plating
workers exposed to soluble $\mathrm{Cr}(\mathrm{VI})$. J. Environ. Monit. 12 (2), 442-447.

Goldoni, M., Corradi, M., Mozzoni, P., Folesani, G., Alinovi, R., Pinelli, S., Andreoli, R., Pigini, D., Tillo, R., Filetti, A., Garavelli, C., Mutti, A., 2013. Concentration of exhaled breath condensate biomarkers after fractionated collection based on exhaled CO2 signal. J. Breath Res. 7 (1), 017101.

Gube, M., Ebel, J., Brand, P., Goeen, T., Holzinger, K., Reisgen, U., Kraus, T., 2010. Biological effect markers in exhaled breath condensate and biomonitoring in welders: impact of smoking and protection equipment. Int. Arch. Occup. Environ. Health 83 (7), 803-811.

Health \& Safety Executive, 2011. EH40/2005 Workplace exposure limits. http:// www.hse.gov.uk/pubns/priced/eh40.pdf/ (Accessed 23 June 2016).

Health \& Safety Executive, 2013. Control of substances hazardous to health (COSHH). The control of substances hazardous to health regulations 2002 (as amended). http://www.hse.gov.uk/pUbns/priced/15.pdf/ (Accessed 14 July 2016).

Health \& Safety Executive, 2013. Chromium and you: Working with chromium Are you at risk? http://www.hse.gov.uk/pubns/indg346.pdf/ (Accessed 23 June 2016)

Hoet, P., 2005. Speciation of chromium in occupational exposure and clinical aspects. In: Cornelis, R., Caruso, J.A., Crews, H., Heumann, K. (Eds.), Handbook of Elemental Speciation II: Species in the Environment, Food, Medicine and Occupational Health. Wiley-Blackwell, pp. 136-157.

Hoffmeyer, F., Weiss, T., Lehnert, M., Pesch, B., Berresheim, H., Henry, J., Raulf-Heimsoth, M., Broding, H., Buenger, J., Harth, V., Bruening, T., 2011. Increased metal concentrations in exhaled breath condensate of industrial welders. J. Environ. Monit. 13 (1), 212-218.

Hoffmeyer, F., Van, K.V., Deckert, A., Neumann, H.D., Buxtrup, M., Willer, E., Felten, C., Bruning, T., Raulf, M., Bunger, J., 2015. Evaluation of airway inflammation in compost workers exposed to bioaerosols using exhaled breath condensate and fractional exhaled nitric oxide. Adv. Exp. Med. Biol. 858, 57-67.

Hulo, S., Cherot-Kornobis, N., Howsam, M., Crucq, S., De Broucker, V., Sobaszek, A. Edme, J.L., 2014. Manganese in exhaled breath condensate: a new marker of exposure to welding fumes. Toxicol. Lett. 226 (1), 63-69.

Hulo, S., Radauceanu, A., Cherot-Kornobis, N., Howsam, M., Vacchina, V., De Broucker, V., Rousset, D., Grzebyk, M., Dziurla, M., Sobaszek, A., Edme, J.L., 2016. Beryllium in exhaled breath condensate as a biomarker of occupational exposure in a primary aluminium production plant. Int. J. Hyg. Environ. Health 219 (1), 40-47.

International Agency for Research on Cancer, 2016. Agents Classified by the IARC Monographs, vols. 1-113. http://monographs.iarc.fr/ENG/Classification/latest_ classif.php/ (Accessed 23 June 2016).

Kharitonov, S.A., Barnes, P.J., 2001. Exhaled markers of pulmonary disease. Am. J. Respir. Crit. Care Med. 163 (7), 1693-1722.

Kuban, P., Foret, F., 2013. Exhaled breath condensate: determination of non-volatile compounds and their potential for clinical diagnosis and monitoring. A review. Anal. Chim. Acta 805, 1-18.

Leese, E., Morton, J., Gardiner, H.E., Carolan, V.A., 2016. Development of a method for the simultaneous detection of $\mathrm{Cr}(\mathrm{III})$ and $\mathrm{Cr}(\mathrm{VI})$ in exhaled breath condensate samples using $\mu$ LC-ICP-MS. J. Anal. At. Spectrom. 31 (4), 924-993.

Morton, J., Tan, E., Leese, E., Cocker, J., 2014. Determination of 61 elements in urine samples collected from a non-occupationally exposed UK adult population. Toxicol. Lett. 231 (2), 179-193.

Paustenbach, D.J., Finley, B.L., Mowat, F.S., Kerger, B.D., 2003. Human health risk and exposure assessment of chromium(VI) in tap water. J. Toxicol. Environ. Health A 66 (14), 1295-1339.

Petersen, R., Thomson, J.F., Jorgensen, N.K., Mikkelsen, S., 2000. Half life of chromium serum and urine in a former plasma cutter of stainless steel. Occup. Environ. Med. 57 (2), 140-142.

Rakhunde, R., Deshpande, L., Juneja, H.D., 2012. Chemical speciation of chromium in water: a review. Crit. Rev. Environ. Sci. Technol. 42 (7), 776-810.

Reinhold, P., Knobloch, H., 2010. Exhaled breath condensate: lessons learned from veterinary medicine. J. Breath Res. 4 (1), 017001.

Rosias, P., 2012. Methodological aspects of exhaled breath condensate collection and analysis. J. Breath Res. 6 (2), 027102. 


\section{Chapter 6.}

Exhaled Breath Condensate: A Novel Matrix for Biological Monitoring to Assess Occupational Exposure to Respirable Crystalline Silica

E. Leese, J. Staff, V.A. Carolan and J. Morton. Ann. Work. Exp. Health. (formerly Ann. Occup. Hyg.), 2017, 61(7): 902 - 906. DOI: 10.1093/annweh/wxx047

Published - Government Open Access Licence

https://academic.oup.com/annweh/article/doi/10.1093/annweh/wxx047/3983354/Ex haled-Breath-Condensate-A-Novel-Matrix-for?guestAccessKey=22f42f1c-4e55-4531-

a633-a757658d0ce3 


\title{
Exhaled Breath Condensate: A Novel Matrix for Biological Monitoring to Assess Occupational Exposure to Respirable Crystalline Silica
}

\author{
Elizabeth Leese ${ }^{1,2}$, James F. Staff', Vikki A. Carolan² and Jackie Morton ${ }^{1 *}$
}

${ }^{1}$ Health and Safety Executive, Harpur Hill, Buxton SK17 9JN UK; ${ }^{2 B}$ Biomolecular Sciences Research Centre, Sheffield Hallam University, Howard Street, Sheffield S1 1WB, UK

*Author to whom correspondence should be addressed. Tel: +44-1298-218437; e-mail: jackie.morton@hsl.gsi.gov.uk

Submitted 12 January 2017; revised 15 May 2017; editiorial decision 24 May 2017; revised version accepted 1 June 2017.

\begin{abstract}
Biological monitoring (BM) is a useful way of determining overall exposures to chemical substances; however, in the case of respirable crystalline silica (RCS), this has not been analytically feasible in conventional biological matrices. The aim of this study was to investigate the utility of exhaled breath condensate $(\mathrm{EBC})$ as a potential biological matrix in which to determine exposure to RCS. A small pilot study was undertaken collecting EBC from six quarry workers and six occupationally unexposed persons; the samples were analysed using both single particle inductively coupled plasma mass spectrometry (spICP-MS) and transmission electron microscopy (TEM). The results showed that EBC obtained from the occupationally unexposed persons exhibited low background levels of dissolved silica whilst silica particles of various sizes were present in samples from quarry workers. This is the first study to report EBC as a potential biological matrix that allows differentiation of RCS concentrations between samples from workers and occupationally unexposed controls. The results shown here confirm the presence of RCS in EBC by both spICP-MS and TEM. However, there are difficult analytical challenges still to be overcome before this can be used as a BM method to determine workplace exposure, these are currently being investigated.
\end{abstract}

Keywords: exhaled breath condensate; respirable crystalline silica; single particle ICP-MS; TEM and quarry workers

\section{Introduction}

The hazards and health outcomes of exposure to respirable crystalline silica (RCS) have been well reported. RCS has been classified as a carcinogen when inhaled in its quartz or cristobalite forms by NIOSH (The National Institute for Occupational Safety and Health, 2016), the US National Toxicology Program (National Toxicology Program 2014), and IARC (Group1; IARC 2016). Occupational exposure to RCS induces distinctive lung toxicity in the form of silicosis (a fibrotic pneumoconiosis), which is characterized by pulmonary oedema, interstitial inflammation, fibrotic granuloma, and silicotic nodules (Kawasaki 2015).

Biological effect markers of exposure, such as elevated levels of inflammatory cytokines, have been

(C) Crown copyright 2017

This Open Access article contains public sector information licensed under the Open Government Licence v3.0 (http://www.nationalarchives.gov.uk/doc/ open-government-licence/version/3/). 
reported as result of exposure to RCS (Kawasaki 2015). The use of biological monitoring (BM) allows the assessment of all routes of exposure and can subsequently help control and reduce exposures to a wide range of chemicals. This is not the case with silica, until now the possibility of a direct BM marker of exposure has eluded researchers. This is as a consequence of the lack of solubility and analytical sensitivity of silica and silicon using spectroscopic methods (resulting from interferences and inherent elevated background levels). Therefore, it has not been possible to use common BM matrices because of the relatively high concentrations of silicon in samples such as urine (Roberts and Williams, 1990; Kobayahsi et al., 1995; Widner et al., 1998) making it difficult to differentiate between workers with potential exposures and unexposed persons. Furthermore, measuring total silicon would not be a direct measurement of RCS, and if an inhalation exposure had occurred it is not known whether or not a urine sample would appropriately reflect this exposure.

Recent developments in instrumental sensitivity and software now offer the potential to measure individual particles of known elemental status. Techniques such as single particle inductively coupled plasma mass spectrometry (spICP-MS) can measure to low concentrations of individual silica particles (as silicon at $\mathrm{m} / \mathrm{z} 28$ ) without the need for aggressive sample digestion (Laborda et al., 2014). In combination with direct particle-sizing instrumentation such as electron microscopy, this offers a comprehensive analytical approach to help identify and quantify the presence of RCS in a suitable biological matrix. Whilst the measurement of silica nanoparticles has been achieved previously using an array of analytical techniques such as asymmetric field flow fractionation, multi-angle light scattering as well as transmission electron microscopy (TEM), spICP-MS, and ICP-MS/ MS (Aureli et al., 2015; Barahona et al., 2015; Bartczak et al., 2015); there are no reports of RCS being analysed in biological samples in this way.

Exhaled breath condensate (EBC) may be an appropriate 'biological matrix' for biomonitoring purposes for RCS in workplace exposures where silica particles are being inhaled. An EBC sample is composed of mostly water vapour and droplets of epithelial lung lining fluid from the bronchial and alveolar regions of the lungs (Hoffmeyer et al., 2011).

The novel work presented here investigates the measurement of RCS in EBC samples from a small cohort of sandstone quarry workers and occupationally unexposed controls (laboratory workers) with analysis by spICP-MS and TEM with energy dispersive X-ray spectroscopy (EDX).

\section{Method}

\section{Sample collection}

The study group consisted of six workers from a sandstone quarry in North Yorkshire, England, and six control volunteers from the Health and Safety Executive's Health and Safety Laboratory who were not occupationally exposed. All participating volunteers gave informed consent in accordance with HSG 167 (HSE, 1997).

EBC samples were collected using a TURBO-DECCS (Transportable Unit for Research on Biomarkers Obtained from Disposable Exhaled Condensate Collection Systems) by ItalChill (Parma, Italy). The TURBO-DECCS technique is as previously described (Leese et al., 2016). The collection of EBC is by regular, calm tidal breathing through the mouth via a disposable mouthpiece for 15 minutes. The exhaled air produced passes into a temperature-controlled chamber where the EBC is cooled and collected as a liquid condensate on the surface of a collection tube. EBC samples from both the quarry workers and the control group were frozen at $-20^{\circ} \mathrm{C}$ immediately after collection and stored at $-80^{\circ} \mathrm{C}$ at the laboratory until analysed. The collection of EBC results in very small sample volumes, usually $<1 \mathrm{ml}$. Therefore, not all EBC samples could be analysed by both spICP-MS and TEM analysis.

A commercially available Health and Safety Laboratory RCS certified reference material (CRM) (89\% certified crystallinity) HSL A9950 (Respirable $\alpha$-Quartz powder) was analysed by all methods. This was prepared with ultra-pure deionized water and diluted to various concentrations depending on the analytical technique.

\section{Analysis spICP-MS}

Analysis was performed on an ICAP Q ICP-MS (Thermo Scientific, Hemel Hempstead, UK) using kinetic energy discrimination (KED) mode with helium as the collision cell gas. The analytical method and sample preparation was performed as outlined in RIKILT's spICP-MS procedure (RIKILT Wageningen UR, https://www. wur.nl/en/show/Single-Particle-Calculation-tool.htm, accessed 17 November 2016). Dwell times were $3 \mathrm{~ms}$ for both ${ }^{197} \mathrm{Au}$ and ${ }^{28} \mathrm{Si}$; sample acquisition was for $60 \mathrm{~s}$. The nebulisation efficiency was calculated at $6.5 \%$ by determining the sample flow rate $(0.24 \mathrm{ml} / \mathrm{min})$ using a 30 nm gold nanoparticle reference material (NIST 8012). All EBC samples were diluted 10-fold with ultra-pure deionized water. The time-resolved data for both the gold and silicon analyses were exported into the RIKILT single particle calculation tool (RIKILT Wageningen UR, https://www.wur.nl/en/show/Single-Particle-Calculationtool.htm, accessed 17 November 2016). 
Transmission electron microscopy

Analysis was performed using a Tecnai G2 Spirit $120 \mathrm{kV}$ TEM (FEI, Cambridge, UK), with attached X-MAX energy dispersive spectroscopy (EDX) system (Oxford Instruments, Abingdon, UK). For each sample, $20 \mu \mathrm{l}$ was dropped onto an ultrathin carbon-coated 200-mesh nickel grid (Agar Scientific, Stansted, UK) and air dried. Electron micrographs were captured at $\times 30000$ plate magnification (approximately $\times 300000$ including magnification by the camera). Particles were analysed by EDX for elemental composition and an electron diffraction (ED) pattern obtained to confirm crystallinity

\section{Results and Discussion}

As shown in Fig. 1, there was an obvious difference between the spICP-MS time scans for the control EBC sample and the quarry worker EBC sample, whilst the time scan of the RCS CRM appeared visibly similar to the quarry worker EBC sample.
Exporting the ICP-MS data into the RIKILT spreadsheet showed that it was possible to determine the number of silica particles in control EBC, worker EBC, and RCS CRM samples. Using this approach over a concentration range of $1-100 \mu \mathrm{g} / \mathrm{l}$ of the RCS CRM, the number of particles showed an incremental increase, from 2 to 245 particles. Results showed a significant difference between the number of particles detected in control EBC samples compared with worker samples. The number of particles detected in workers' EBC ranged from 11 to 354 whilst the control samples showed between 0 and 8 particles. The EBC sample from the quarry worker cited in Fig. 1 contained 354 particles with particles ranging in size from $\sim 300$ to $1200 \mathrm{~nm}$.

There are limitations of the spICP-MS approach for RCS determinations and this impact upon the accurate quantitative reporting of particle size. Firstly, there are no commercially available certified standard or reference materials of size-characterized crystalline silica particles to fully validate the spICP-MS method. Sec-

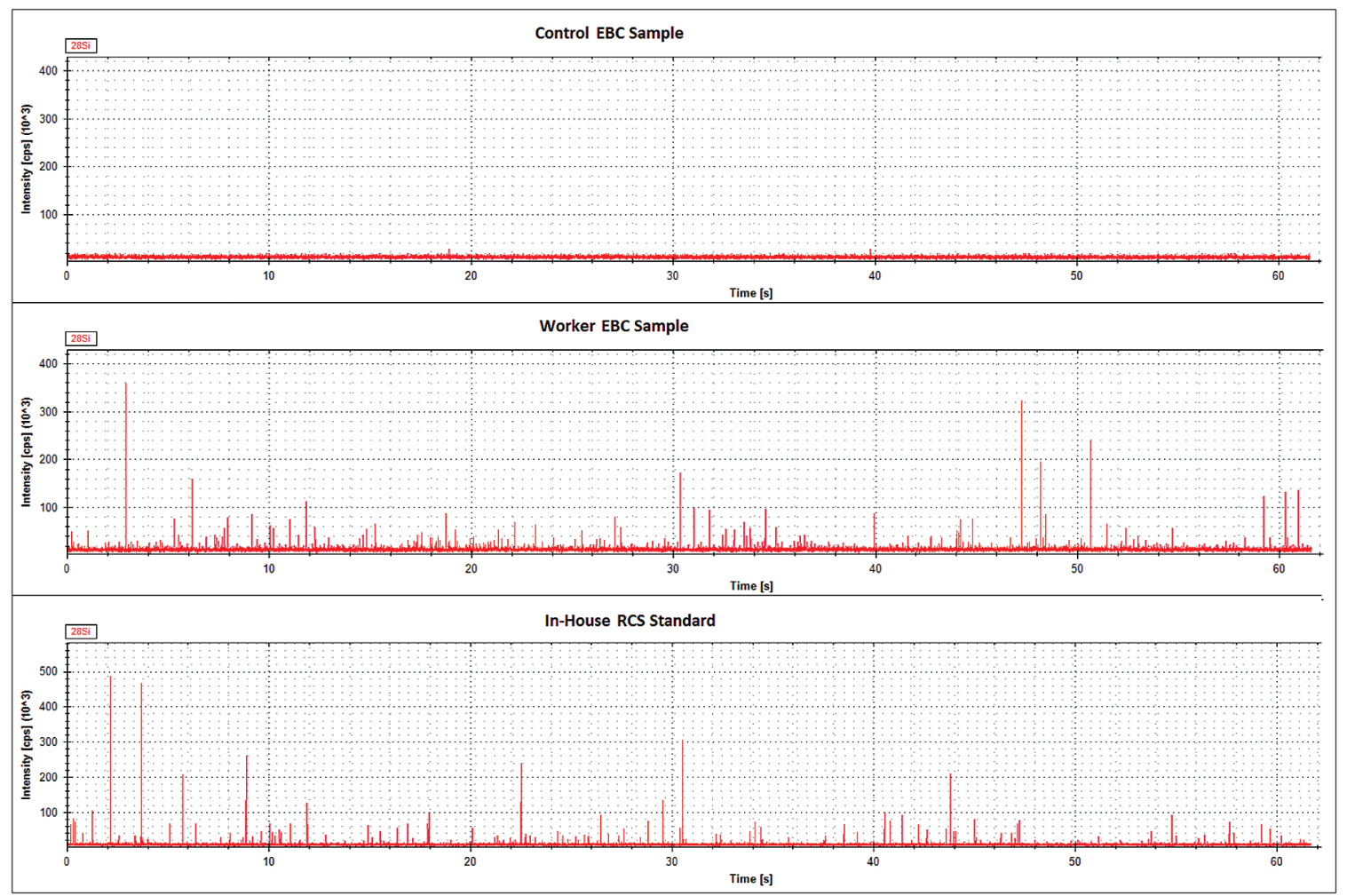

Figure 1. Time scan of silica particles as ${ }^{28} \mathrm{Si}$ in EBC samples from (A) occupationally unexposed control (B) quarry worker (C) RCS CRM 50 $\mu \mathrm{g} / \mathrm{l}$ (HSL A9950 (Respirable $\alpha$-Quartz powder), when measured by spICP-MS in KED mode. Note that the single particle peaks are detected randomly throughout the 1-minute sample acquisition and so the scans will not be replicated. 
ondly, the RIKILT calculations (RIKILT Wageningen UR, https://www.wur.nl/en/show/Single-Particle-Calculation-tool.htm, accessed 17 November 2016) assume spherical particles, but RCS is neither spherical nor a consistent shape and so all references to particle size using this approach at this point only give a qualitative value. Finally, the current limit of size detection using single quadruple ICP-MS is estimated to be around $300 \mathrm{~nm}$.

TEM was able to confirm the presence of RCS in EBC from the quarry workers in all four post-shift sam- ples analysed. No RCS was observed by TEM in EBC samples from the control samples.

Using TEM, analysis of an EBC sample from the quarry worker with the most particles detected by spICP-MS exhibited 10 crystalline silica particles ranging in diameter from $100 \mathrm{~nm}$ to $9000 \mathrm{~nm}$ (one is shown in Fig. 2). One particle was observed approximately every 5-10 grid openings inspected.

It is important to note that not all the silicon containing particles observed with TEM were crystalline silica $\left(\mathrm{SiO}_{2}\right)$. Using EDX, it was possible to identify some par-
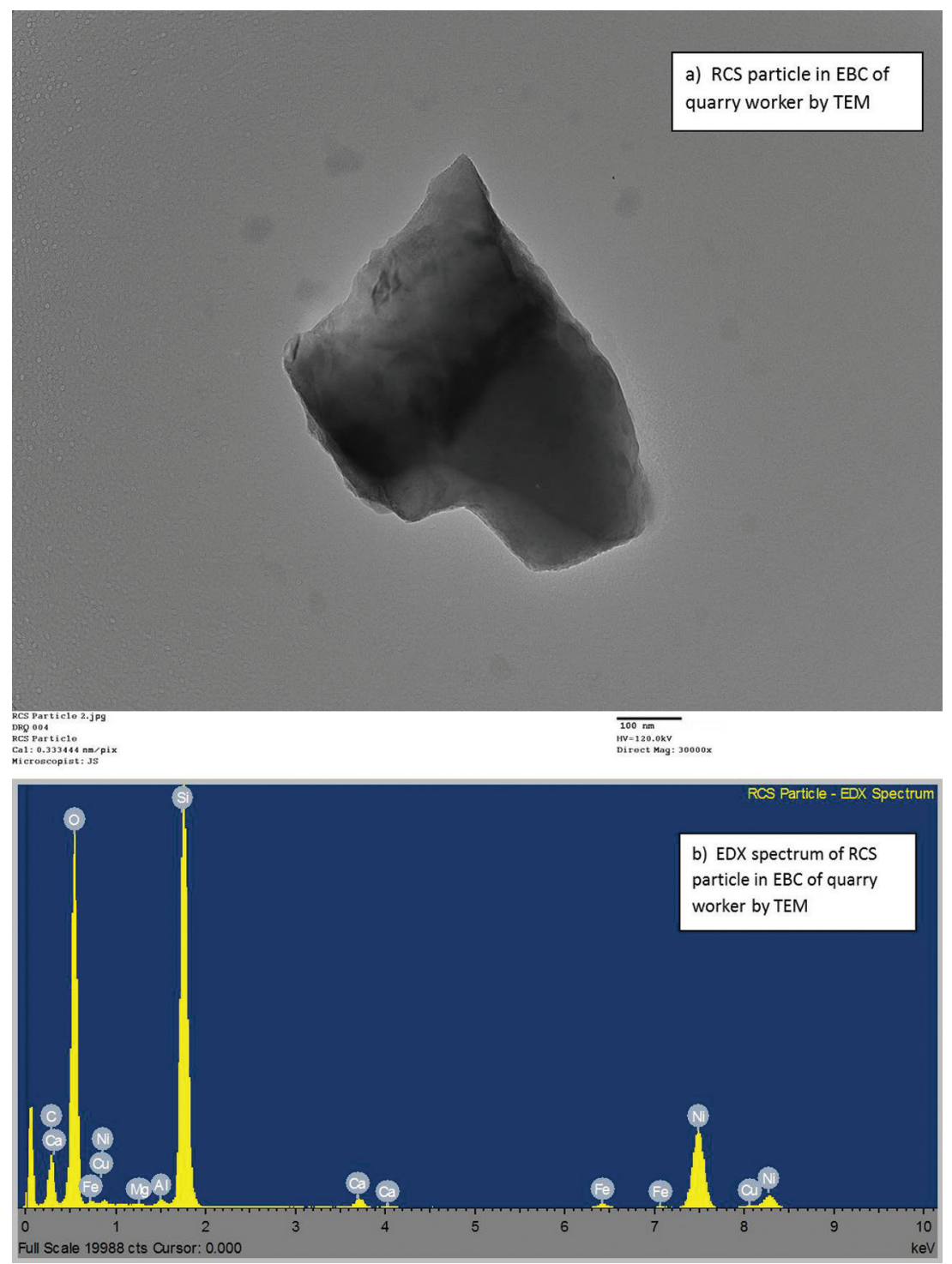

Figure 2. (a)TEM micrograph of a RCS particle observed in an EBC sample from a quarry worker (same sample as seen in Fig. 1), confirmed as silica by EDX (b). 
ticles as aluminium silicates. This information is helpful in identifying the composition of the 'baseline' observed with spICP-MS, i.e. the contribution of other silicates as well as amorphous silica. Another contributing factor is likely to be from the quartz background of the ICPMS sample introduction system, residual contamination/ impurities of all solutions including standards and of the argon gas used in the plasma.

\section{Conclusion}

This work demonstrates that it is possible to detect crystalline silica particles in EBC of quarry workers. However, as the collection of EBC only produces a small volume of sample we need to be able to acquire a lot of information efficiently. At this stage, the analysis and characterisation of the silica particles still require further method development. There are several analytical challenges to overcome; specifically that when using spICPMS, the particles are easily detected but not sized; and with the current TEM methodology, a large sample area must be searched to provide high analytical sensitivity. Future developments of these methods will include sample pre-concentration methods for the TEM and the use of a shorter dwell time in spICP-MS. The removal of interferences using ICP-MS/MS may also offer further analytical improvements. It may also be worth considering calculations using particle mass instead of size, or determining the hydrodynamic radius of RCS particles in solution.

The potential of EBC as a matrix for determining exposure to RCS has been realized. It is now likely that future EBC measurements will be able to show actual worker exposures and assist with the improvement of workplace controls.

\section{Funding}

This publication and the work it describes were funded by the Health \& Safety Executive (HSE).

\section{Declaration}

Its contents, including any opinions and/or conclusions expressed, are those of the authors alone and do not necessarily reflect HSE policy.

\section{References}

Aureli F, D'Amato M, Raggi A et al. (2015) Quantitative characterization of silica nanoparticles by asymmetric flow field flow fractionation coupled with online multiangle light scattering and ICP-MS/MS detection. J Anal At Spectrom; 30: 1266-73.

Barahona F, Geiss O, Urbán P et al. (2015) Simultaneous determination of size and quantification of silica nanoparticles by asymmetric flow field-flow fractionation coupled to ICPMS using silica nanoparticles standards. Anal Chem; 87: 3039-47.

Bartczak D, Vincent P, Goenaga-Infante H. (2015) Determination of size- and number-based concentration of silica nanoparticles in a complex biological matrix by online techniques. Anal Chem; 87: 5482-5.

Hoffmeyer F, Weiss T, Lehnert M et al. (2011) Increased metal concentrations in exhaled breath condensate of industrial welders. J Environ Monitor; 13: 212-8.

HSE. (1997) Available at http://www.hse.gov.uk/pubns/priced/ hsg167.pdf. Accessed 12 December 2016.

IARC. (2016) Agents classified by the IARC monographs, volumes 1-113. Available at http://monographs.iarc.fr/ENG/ Classification/latest_classif.php. Accessed 22 August 2016.

Kawasaki HA. (2015) mechanistic review of silica-induced inhalation toxicity. Inhal Toxicol; 27: 363-77.

Kobayahsi R, Imaizumi K, Kudo M. (1995) Determination of silicon in urine by graphite-furnace atomic absorption spectrometry with a nickel chloride modifier. Anal Sci; 11; 267-9.

Laborda F, Bolea E, Jiménez-Lamana J. (2014) Single particle inductively coupled plasma mass spectrometry: a powerful tool for nanoanalysis. Anal Chem; 86: 2270-8.

Leese E, Morton J, Gardiner HE et al. (2016) Development of a method for the simultaneous detection of $\mathrm{Cr}(\mathrm{III})$ and $\mathrm{Cr}(\mathrm{VI})$ in exhaled breath condensate samples using $\mu \mathrm{LC}-\mathrm{ICP}-\mathrm{MS}$. Anal At Spectrom; 31: 924-93.

National Toxicology Program (NTP). (2014) Report on Carcinogens. US Department of Health \& Human Services. 13th edn. US Department of Health \& Human Services. Available at https://ntp.niehs.nih.gov/pubhealth/roc/ index-1.html. Accessed 22 August 2016.

Roberts NB, Williams P. (1990) Silicon measurement in serum and urine by direct current plasma emission spectrometry. Clin Chem; $36(8$ Pt 1): 1460-5.

The National Institute for Occupational Safety and Health (NIOSH). (2012) Occupational Cancer - Carcinogens List. NIOSH. Available at http://www.cdc.gov/niosh/topics/cancer/npotocca.html. Accessed 22 August 2016.

Widner B, Mirlach A, Hausen A et al. (1998) Determination of silicon in urine by inductive coupled plasma-optical emission spectroscopy. Clin Chim Acta; 277: 51-63. 


\section{Chapter 7. Discussion \& Conclusion}


This discussion will examine and review the work outlined in Chapters 2 and 3 for arsenic speciation, Chapters 4 and 5 for chromium speciation in EBC and Chapter 6 for single particle analysis of silica.

\subsection{Arsenic Speciation}

Since the development of the arsenic speciation methodology using an Elemental Scientific oneFAST system coupled to an ICP-MS with a Dionex AG7 anion exchange column, as outlined in Chapter 2 of this thesis, it has become one of the Health \& Safety Laboratory's (HSL) routine assays. It has been featured as an "analytical service" since 2012 and, to date, HSL have analysed over 2500 urine samples for customers around both the UK and the world, generating approximately $f 24 \mathrm{~K}$ of annual revenue. With biological monitoring as the focus, the majority of customers are industry based, with samples routinely sent from semi-conductors workers, glass manufacture workers, infrared lens manufacture workers, miners, quarry workers and waste treatment, disposal and recycling industry workers. However, being the only laboratory in England to offer arsenic speciation as an analytical service has led to numerous samples being received from the NHS Trace Element Supra-Regional Assay Service (SAS) Laboratories, of which several are located around the UK. As with most commercial laboratories, the NHS laboratories lack the instrument capacity, time and/or expertise for a dedicated routine speciation service. Urine samples which are flagged as having an elevated total arsenic content during multi-elemental screening are sent to the HSL for arsenic speciation. One sample received from the SAS laboratory at Glasgow Royal Infirmary was from a hospital patient who suspected they had been poisoned with arsenic, which unfortunately resulted in being fatal for the patient. Speciation analysis indicated high concentrations of inorganic arsenic and methylated metabolites, in addition to a very large unexpected peak in the data. The investigation outlined in Chapter 3 identified this peak as thio-DMA. This resulted in a co-authored publication with Andy Duncan (the then, Glasgow SAS Trace Element Director) as a homicidal arsenic poisoning case study, which can be found in Appendix A. 
The work described in Chapter 2 demonstrates a fast, reliable and reproducible arsenic speciation method to fully separate five arsenic species $\left(\mathrm{As}^{3+}, \mathrm{As}^{5+}, \mathrm{MMA}, \mathrm{DMA}\right.$ and $\left.\mathrm{AB}\right)$ in urine in under 6 minutes. ${ }^{243}$ It highlights the need for speciation analysis due to different foodstuffs elevating methylated arsenic species in the urine of a population not occupationally exposed. The study reported that people who had consumed fish within the previous seven days had urinary DMA concentrations twice as high as those who had not eaten fish, which is in direct agreement with another UK study containing a non-occupationally exposed control group. ${ }^{157}$ It was also demonstrated that urinary MMA concentrations were twice as high for those who had consumed fish over those who had not, with red wine being a significant contributor to elevated urinary $A B$ concentrations. The study also established for the first time in the UK the valuable $95^{\text {th }}$ percentile background unexposed reference ranges for each of the five species of arsenic, which is now incorporated into all customer reports of samples analysed as part of the HSLs analytical service. Table 12 outlines the $95^{\text {th }}$ percentile of the five arsenic species obtained in Chapter 2 of this thesis in both $\mu \mathrm{g} / \mathrm{g}$ of creatinine and uncorrected for creatinine $(\mu \mathrm{g} / \mathrm{L}$ ), (the results have been converted from $\mu \mathrm{mol} / \mathrm{mol}$ creatinine to $\mu \mathrm{g} / \mathrm{g}$ creatinine for comparison purposes). In addition, values reported in several recent worldwide occupationally unexposed population studies in both uncorrected for creatinine ( $\mu \mathrm{g} / \mathrm{L}$ ) and $\mu \mathrm{g} / \mathrm{g}$ creatinine where available are included. It highlights the different urinary arsenic species of a range of populations and communities due to dietary variations. The $95^{\text {th }}$ percentile of urinary inorganic arsenic concentrations are relatively low in the UK, ${ }^{243}$ Germany, ${ }^{244}$ U.S. $^{211}$ and Spain, ${ }^{245}$ compared against the $95^{\text {th }}$ percentile of urinary inorganic arsenic concentrations for a Korean community living within the U.S., which was $10.8 \mu \mathrm{g} / \mathrm{g}$ of creatinine. ${ }^{158}$ Only the $75^{\text {th }}$ percentile was reported for Japanese urinary $\mathrm{As}^{3+}$ concentrations, which was 5.8 $\mu \mathrm{g} / \mathrm{g}$ of creatinine. ${ }^{246}$ The $95^{\text {th }}$ percentile would be even higher. In contrast, urinary $D M A$ and $A B$ concentrations were vastly different amongst the different populations, with the Spanish population study showing the highest $95^{\text {th }}$ percentile urinary DMA concentration of $1153.9 \mu \mathrm{g} / \mathrm{g}{ }^{245}$ 
Table 12 The $95^{\text {th }}$ and $75^{\text {th }}$ percentiles of different arsenic species reported in population studies around the World.(P\% denotes the percentile of the population)

\begin{tabular}{|c|c|c|c|c|c|c|c|}
\hline$\mu g / g$ creatinine corrected & $\mathbf{P} \%$ & $\mathrm{As}^{3+}$ & $\mathrm{As}^{5+}$ & MMA & DMA & $\mathbf{A B}$ & Ref \\
\hline UK (Chapter 2 of this thesis) & 95 & 0.66 & 0.23 & 2.04 & 10.7 & 115.7 & 243 \\
\hline Germany & 95 & 0.65 & 1.1 & 2.3 & 15.8 & 23.3 & 244 \\
\hline U.S. & 95 & 1.62 & $<L O D$ & 1.61 & 12.7 & 38.7 & 211 \\
\hline Canada & 95 & - & $<L O D$ & 1.7 & 20 & 44 & 247 \\
\hline Spain & 95 & - & - & - & 286.3 & 1153.9 & 245 \\
\hline U.S. Korean Community & 95 & \multicolumn{2}{|c|}{10.8} & 6.0 & 77.0 & - & 158 \\
\hline Japan & 75 & 5.8 & 1.1 & 4.0 & 47.9 & 88.5 & 246 \\
\hline UK Bangladeshi Community & 75 & \multicolumn{2}{|c|}{0.81} & 0.88 & 25.3 & - & 248 \\
\hline $\begin{array}{l}\mu \mathrm{g} / \mathrm{L} \text { uncorrected for } \\
\text { creatinine }\end{array}$ & $\mathbf{P} \%$ & $\mathrm{As}^{3+}$ & $\mathrm{As}^{5+}$ & MMA & DMA & $\mathbf{A B}$ & Ref \\
\hline UK (Chapter 2 of this thesis) & 95 & 0.54 & 0.23 & 2.37 & 12.68 & 126.7 & 243 \\
\hline Germany & 95 & 0.49 & 0.53 & 1.6 & 9.1 & 22.7 & 244 \\
\hline U.S. & 95 & 1.19 & $<L O D$ & 1.53 & 11.8 & 30.5 & 211 \\
\hline Canada & 95 & - & $<L O D$ & 1.5 & 16 & 56 & 247 \\
\hline Spain & 95 & $<L O D$ & $<L O D$ & 1.8 & 31.9 & 312.8 & 245 \\
\hline U.S. Korean Community & 95 & \multicolumn{2}{|c|}{9.7} & 6.5 & 62.2 & - & 158 \\
\hline Japan & 75 & 8.9 & 1.1 & 5.5 & 65.9 & 131.8 & 246 \\
\hline
\end{tabular}

The results for each population study in Table 12 highlight the incidence of increased methylated metabolites from dietary sources and the need for speciation analysis. It also highlights the need for a comprehensive study into the arsenic species found in different foods. As different countries in the world (in particular the UK) become more 
diverse in terms of the population itself, in addition to the importation and availability of worldwide foods, there is a clear need in understanding background unexposed populations when trying to interpret urinary occupational exposure. In addition, the incidence of different arsenic species to the five traditional species of biological monitoring, such as the presence of arsenolipids, arsenosugars and alternative DMA and MMA analogues, will likely increase. A very recent publication by Carioni et $a l^{249}$ built on the published study outlined in Chapter $2 .{ }^{243}$ They developed the method further to include the arsenic species $A C$ and TMAO, reducing separation time to five minutes. Unfortunately, they were unable to separate TMAO from $A B$ and reported this as a combined value. Additionally, due to possible co-elution of $\mathrm{As}^{3+}$ with either DMA or $A C$, urine samples were treated with hydrogen peroxide $\left(\mathrm{H}_{2} \mathrm{O}_{2}\right)$ to oxidise all $\mathrm{As}^{3+}$ to $\mathrm{As}^{5+}$ and subsequently they reported a combined inorganic arsenic result. However, this method would not be suitable for urine samples containing possible thioarsenicals, as adding $\mathrm{H}_{2} \mathrm{O}_{2}$ to convert $\mathrm{As}^{3+}$ to $\mathrm{As}^{5+}$ would also oxidise any thioarsenicals in the urine samples to their oxoarsenical pairs. In the case of thio-DMA, oxidation would result in an increased urinary DMA concentration with no knowledge that the thio-DMA was present.

The analysis of so many samples at the HSL sourced from different industries, population groups and locations around the globe has led to the detection of unexpected arsenic peaks within several urine samples. The focus of Chapter 3 in this thesis is to investigate two unexpected arsenic peaks found in various urine samples. One peak has been identified as thio-DMA, however the second remains unknown. Nevertheless, the work has established that it is not thio-DMA, DMAA, thio-DMAA, DMAE, thio-DMAE, AC or TMAO. Speciation analysis has shown that the unidentified peak is not related to inorganic occupational exposure and so must be related to environmental exposure, most likely from a dietary source. The primary difficulty with this type of analytical investigation is the lack of commercially available standards (other than inorganic arsenic, $\mathrm{DMA}^{5+}, \mathrm{MMA}^{5+}, \mathrm{AB}, \mathrm{AC}$ and TMAO) to aid identification and quantification of arsenic compounds. This also applies to certified reference materials (CRM) with ClinChek $^{\circledR}$ Controls 8931 (Recipe, Germany) providing two urinary levels for AB, DMA, MMA and $\mathrm{As}^{5+}$ only, NIST 3669 (Gaithersburg, U.S.) 
providing an urinary elevated arsenic level for $\mathrm{AB}, \mathrm{DMA}, \mathrm{MMA}, \mathrm{As}^{5+}$ and $\mathrm{As}^{3+}$, in addition to NIST 2669 also including urinary levels for TMAO and AC. There are no commercially available calibration quality standard materials or urinary CRM for other methylated metabolites, including DMA analogues such as thio-DMA in addition to arsenosugars. To enable further identification of arsenic species and continued research in the field, this lack of availability needs to be addressed. It is highly likely that thio-DMA and other DMA analogues will be routinely observed in urine samples and as such will need to be accurately measured and become part of a routine biological monitoring speciation method.

A greater understanding of the variety of arsenic species in food also needs to be developed. For biological monitoring, it would be useful to primarily target different food stuffs as possible sources of dietary exposure when explaining and interpreting speciation results to customers. However, it is also necessary in the broader field to help characterise sources of exposure in diet for risk assessment and toxicological purposes. Figure 16 tries to simplify the routes of exposure and the currently known expected or possible metabolites from inorganic arsenic, arsenolipid, arsenosugar and arsenobetaine exposure. The range of these expected metabolites is likely to expand as further research continues and analytical technology carries on improving. For example, a study by Molin et $\left.a\right|^{250}$ reported the urinary excretion of seventeen different arsenic compounds (six of which were thioarsenicals) in a volunteer study after identical ingestions of blue mussels, but with vastly different inter-individual excretionary patterns. This highlights the possibility of an extensive number of arsenic compounds found in urine. 


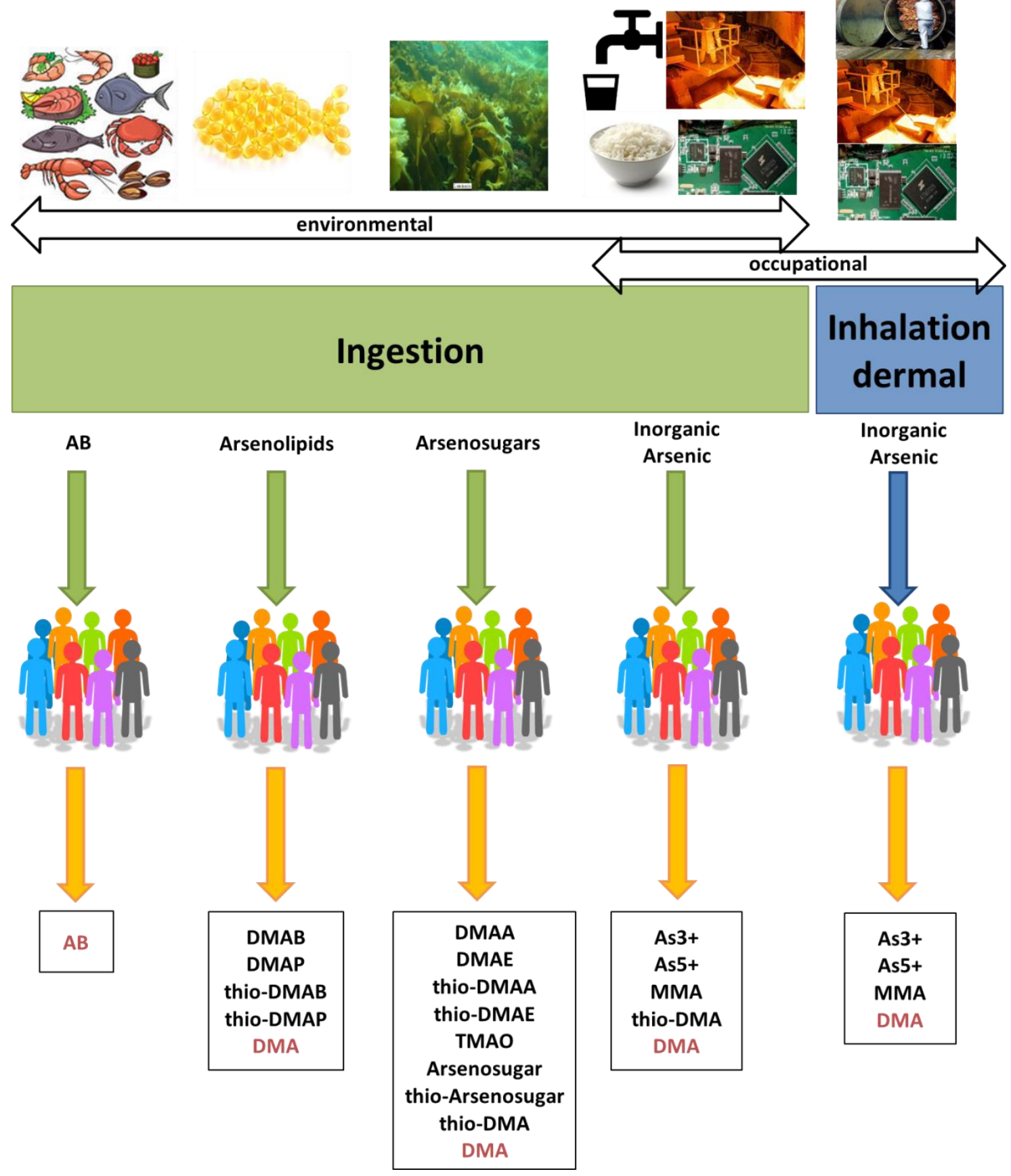

Figure 16 Sources of different categories of arsenic exposure and the resulting metabolites. Red compounds indicate the major metabolite from each source of exposure

It is important to note that, to date, inorganic arsenic exposure by either dermal absorption or inhalation has yet to be directly linked to the DMA analogues such as thio-DMA and has only been directly related to dietary inorganic exposure including drinking inorganic arsenic contaminated water. A reason for this may be that the 
formation of DMA analogues, especially thioarsenicals is reliant on conditions unique to the stomach or gut. The in vitro formation of thioarsenicals from gut flora has been demonstrated in rats ${ }^{251}$ and mice, ${ }^{252,253}$ directly linking the importance of ingestion with the production of thio-DMA and other DMA analogues. However, the production of thio-DMA was demonstrated in $\mathrm{RBC}^{156,254}$ and most recently in human liver cells, ${ }^{146}$ indicating the gut may not be the sole site of thioarsenical formation. It is also interesting to note that $\mathrm{DMA}^{5+}$ is the major urinary metabolite for all arsenic compounds, which can be metabolised by humans.

Recent in vitro findings have determined that some organic arsenic compounds and their metabolites are cytotoxic, ${ }^{142,145}$ with other organic compounds, such as DMAA and DMAE in addition to their thio pairs, being neither cytotoxic or genotoxic. ${ }^{142}$ Though cytotoxic, some toxicity studies have determined that the cellular toxic modes of action differ from those of arsenite. ${ }^{145,150}$ Arsenite's cellular toxic mode of action is genotoxic related, due to multiple DNA lesions and strand breakages. ${ }^{145}$ The variability in toxicity amongst the organic arsenic metabolites suggests that further toxicological investigations are required. It is acknowledged by the scientific community that there is a knowledge gap in understanding exposures, the transformation of species as well as the toxicokinetics and metabolic pathways of arsenic species in humans, and the individual variability of organic arsenic excretion. ${ }^{130,135,255}$

Future work at the HSL will be to identify the unexpected arsenic compound known as peak 2, in the work reported in Chapter 3. This could involve the commencement of human dosing volunteer studies, to ingest known foods identified in the literature which have resulted in the metabolism and excretion of alternative arsenic compounds to those currently measured at the HSL and those investigated in Chapter 3 of this thesis. Food items already selected from the literature include cod liver oil, mussels and seaweed sources. This will help to identify dietary sources commercially available in the UK that might result in unexpected arsenic peaks in future biological monitoring urinary samples. In addition, this tactic may also result in an unexpected peak at the same retention time as peak 2 which will produce fresh urine samples for further identification purposes but also help narrow down the identity of the arsenic 
compound if it has been previously metabolised and excreted in a specific ingestion study.

\subsection{Chromium Speciation}

The work described in Chapter 4 and Chapter 5 of this thesis, demonstrates a method to simultaneously detect and measure both trivalent $(\mathrm{Cr}(\mathrm{III}))$ and hexavalent $(\mathrm{Cr}(\mathrm{VI}))$ chromium in EBC samples. Chapter 4 outlines the development of the analytical method, including a storage stability study of spiked solutions of $\mathrm{Cr}(\mathrm{III})$ and $\mathrm{Cr}(\mathrm{VI})$ in EBC. Chapter 5 describes a feasibility study to determine whether $\mathrm{Cr}(\mathrm{III})$ and $\mathrm{Cr}(\mathrm{VI})$ can be detected in 'real' EBC samples from workers, using the method developed in Chapter 4. The feasibility study demonstrated for the first time that it was possible to measure and detect both species of chromium in occupationally exposed workers, with workers showing significantly higher levels of both $\mathrm{Cr}(\mathrm{III})$ and $\mathrm{Cr}(\mathrm{VI})$ in their EBC samples compared to the control group, in addition to higher urinary total chromium levels. Site visits consisted of collecting both pre and post working week urine and EBC samples. However, despite differences in urine samples, no significant difference was found between pre and post working week EBC samples. The work outlined in Chapter 5 was one of the top 30 most downloaded articles published in the Journal of Analytical Atomic Spectrometry (JAAS) in 2016 and has been included in the JAAS 2016 Most Accessed Articles Collection.

Little is known about the mechanisms or EBC in terms of its effectiveness for exposure assessment and so using EBC as a matrix for biological monitoring raises numerous questions that cannot as yet be fully answered. Exact inhalation deposition rates of inhaled elemental particles/fumes/vapour and mucociliary clearance abilities from the lungs are undefined. Therefore, how much an individual inhales of hexavalent chromium for example in comparison to how much is then exhaled and measured in an EBC sample remains inconclusive. Further work at the HSL will involve site visits to companies with workers occupationally exposed to $\mathrm{Cr}(\mathrm{VI})$ by inhalation to repeat $\mathrm{EBC}$ sampling in tandem with personal air measurements. This will enable the investigation of whether there is a correlation of $\mathrm{Cr}(\mathrm{VI})$ air levels with levels exhaled in $\mathrm{EBC}$ samples. 
In addition, it is also unknown whether the $\mathrm{Cr}$ (III) measured in the worker's EBC samples was from reduction of $\mathrm{Cr}(\mathrm{VI})$ in the lungs or whether some contribution is from inhaled $\mathrm{Cr}(\mathrm{III})$ as a result of reduction from $\mathrm{Cr}(\mathrm{VI})$ in the environment of the workplace. The measurement of both $\mathrm{Cr}(\mathrm{III})$ and $\mathrm{Cr}(\mathrm{VI})$ from personal air samplers may help to answer this question. Other valid questions include:

- what are EBC measurements representative of, the previous five minutes of exposure, five weeks or five years?

- how long can $\mathrm{Cr}(\mathrm{VI})$ be measured in an EBC sample after exposure has ceased?

These questions will be valid in the study of silica in EBC samples also, arising from the work outlined in Chapter 6.

As previously stated, the feasibility study in Chapter 5 found no significant difference in $\mathrm{EBC} \mathrm{Cr}(\mathrm{VI})$ levels between pre and post working week samples. However, this is not the case for other studies. A study by Goldoni et al, ${ }^{65}$ determining $\mathrm{Cr}(\mathrm{VI})$ only in EBC samples of 10 chrome platers, determined $\mathrm{Cr}(\mathrm{VI})$ in EBC samples of 9 of the 10 workers and 4 of those 9 workers had lower levels of $\mathrm{Cr}(\mathrm{VI}) 15$ hours after exposure. An earlier study by the same research group also investigated biomarkers of oxidative stress (hydrogen peroxide $\left(\mathrm{H}_{2} \mathrm{O}_{2}\right)$ and malondialdehyde (MDA)) in addition to $\mathrm{Cr}(\mathrm{VI})$ levels in EBC. $^{66}$ EBC samples were taken pre and post shift on a Friday and again on the following Monday morning. An increase in both $\operatorname{Cr}(\mathrm{VI})$ and the biomarkers was observed between the Friday pre and post shift samples, with detectable but lower levels also observed in the Monday morning EBC sample. Levels of $\mathrm{Cr}(\mathrm{VI})$ were shown to correlate with both $\mathrm{EBC} \mathrm{H}_{2} \mathrm{O}_{2}$ and EBC MDA levels. ${ }^{66}$

Hexavalent chromium is an elemental species of occupational concern to HSE. Chrome plating is a large and widespread industry within the UK, that both the HSE and the HSL have a good professional relationship with, including its professional trade association (Surface Engineering Association). This is mostly due to large scale HSE surveys conducted within the industry over the previous 10 years resulting in collaborative guidance documents and reports. ${ }^{256-261}$ Correlation between $\mathrm{Cr}(\mathrm{VI})$ air measurements and total chromium urinary measurements are already defined, in addition to 
guidance values available from both the U.S. and Germany. This wealth of knowledge of occupational exposure is not known for many other elements, especially crystalline silica exposure. This information, in addition to employers and employees already used to volunteering in $\mathrm{HSL}$ studies, makes $\mathrm{Cr}(\mathrm{VI})$ an ideal element to begin the investigations into the potential of $E B C$ as a new biological matrix for biological monitoring. Although differences are bound to be observed between different compounds and elements, the knowledge gained can be applied to future EBC measurements. This project has the potential to provide further information and evidence of exposure than that previously available and should in time help with controlling exposure.

\subsection{Single Particle Analysis}

The technique of spICP-MS is still in its infancy, with experts in ICP-MS analysis, particle and nanoparticle technology, ICP-MS instrument manufacture and software development all learning together regarding this extremely promising application. There are numerous techniques for measuring single particles or nanoparticles, as listed in Table 13. All have analytical limitations, which has resulted in no technique being the standout choice for single particle analysis. Degueldre and Favarger in $2003^{233}$ presented the feasibility of ICP-MS to measure single particles. This has paved the way in recent years for ICP-MS to begin establishing itself as having the potential to accurately provide particle size, particle number concentration and size distribution in varied liquid matrices at concentrations relevant to environmental or biological systems. It is the only technique that both quantitatively counts particles and

determines the chemical composition of the material. ${ }^{262}$ However, there is still a long way to go if spICP-MS is to establish itself as a preferred analytical technique for single particle or nanoparticle analysis. 
Table 13 A comparison of abilities and limitations of different analytical techniques to measure particles and nanoparticles ${ }^{262-270}$

\begin{tabular}{|c|c|c|c|}
\hline Technique & Strengths / Benefits & Limitations & Used to size, characterise or image \\
\hline spICP-MS & $\begin{array}{l}\text { - } \quad \text { Particle size determination } \\
\text { - } \text { Particle size distribution } \\
\text { - } \quad \text { Particle number concentration } \\
\text { - } \quad \text { Mass Concentration } \\
\text { - } \quad \text { Elemental composition determination } \\
\text { - } \quad \text { Minimal sample preparation required }\end{array}$ & $\begin{array}{l}\text { - } \quad \text { Cannot determine shape } \\
\text { - } \quad \text { Measurement based upon spherical } \\
\text { - } \quad \text { Not capable of multi-element analysis } \\
\text { - } \quad \text { Calibration is based on a mathematical } \\
\text { - } \quad \text { assumption } \\
\text { particle analysis }\end{array}$ & - Metal or metal oxide based particles \\
\hline $\begin{array}{l}\text { Dynamic Light Scattering } \\
\text { (DLS) }\end{array}$ & $\begin{array}{l}\text { - } \quad \text { Fast and simple analysis time } \\
\text { - Uses small sample volumes }\end{array}$ & $\begin{array}{l}\text { - } \text { Poor method sensitivity } \\
\text { - } \quad \text { Cannot distinguish elemental } \\
\text { - } \quad \text { Nomposition } \\
\text { - quantitative }\end{array}$ & $\begin{array}{l}\text { - } \text { Proteins } \\
\text { - } \quad \text { Nanotechnology particles } \\
\text { - } \quad \text { Metal particles }\end{array}$ \\
\hline $\begin{array}{c}\text { Transmission electron } \\
\text { microscopy } \\
\text { (TEM) } \\
\text { Scanning electron microscopy } \\
\text { (SEM) }\end{array}$ & $\begin{array}{l}\text { - } \quad \text { Particle shape determination } \\
\text { - } \quad \text { Particle size determination } \\
\text { - } \quad \text { Ability to resolve agglomerates and } \\
\text { aggregates } \\
\text { - Can determine elemental composition } \\
\text { only when combined with EDX* }\end{array}$ & $\begin{array}{l}\text { - } \quad \text { Unsuited to high throughput } \\
\text { - } \text { High particle count required } \\
\text { - } \quad \text { Poor method sensitivity } \\
\text { - } \quad \text { Not quantitative } \\
\text { - } \quad \begin{array}{l}\text { Sample preparation can induce } \\
\text { agglomeration }\end{array}\end{array}$ & $\begin{array}{l}\text { - } \text { Asbestos and fibres counting } \\
\text { - } \quad \text { Material characterisation } \\
\text { - } \quad \text { Study of crystals and metals } \\
\text { - } \quad \text { Detection of flaws, fractures \& } \\
\text { - } \quad \text { damage in micro sized objects } \\
\text { - } \quad \text { Virus \& bacteria imaging }\end{array}$ \\
\hline
\end{tabular}




\begin{tabular}{|c|c|c|c|}
\hline Technique & Strengths / Benefits & Limitations & Used to size, characterise or image \\
\hline & & & $\begin{array}{ll}\text { - } & \text { Cell biology } \\
\text { - } & \text { Cancer cell imaging }\end{array}$ \\
\hline $\begin{array}{l}\text { Nanoparticle Tracking } \\
\text { Analysis } \\
\text { (NTA) } \\
\text { Particle tracking analysis } \\
\text { (PTA) }\end{array}$ & $\begin{array}{l}\text { - } \quad \text { Particle size distribution } \\
\text { - } \quad \text { Particle size determination } \\
\text { - Particle number concentration } \\
\text { - } \quad \text { Minimal sample preparation required }\end{array}$ & $\begin{array}{l}\text { - } \begin{array}{l}\text { Cannot distinguish elemental } \\
\text { composition }\end{array} \\
\text { - } \quad \text { Struggles with sizes }>500 \mathrm{~nm} \\
\text { - } \quad \text { Measurement based upon spherical } \\
\text { particles } \\
\text { - } \quad \text { Light emitted from large particles will } \\
\text { overlay that of smaller particles } \\
\text { - Unable to distinguish between } \\
\text { particles of small size differences }\end{array}$ & $\begin{array}{l}\text { - } \text { Proteins } \\
\text { - Virus characterisation } \\
\text { - Nanotechnology particles } \\
\text { - Metal particles } \\
\text { - Cell composition }\end{array}$ \\
\hline $\begin{array}{l}\text { Resistive Pulse Sensing (RPS) } \\
\text { Scanning Ion Occlusion } \\
\text { spectroscopy (SIOS) }\end{array}$ & $\begin{array}{l}\text { - } \quad \text { Particle size determination } \\
\text { - } \quad \text { Particle size distribution } \\
\text { - High throughput }\end{array}$ & $\begin{array}{l}\text { - Not suitable for particles suspended in } \\
\text { - } \quad \text { water or organic solvents } \\
\end{array}$ & $\begin{array}{ll}\text { - } & \text { Proteins } \\
\text { - } & \text { Virus characterisation } \\
\text { - } & \text { Nanotechnology particles } \\
\text { - } & \text { Characterisation of drug delivery } \\
& \text { particles } \\
\text { - } & \text { Cell composition }\end{array}$ \\
\hline $\begin{array}{l}\text { Differential Centrifugal } \\
\text { Sedimentation } \\
\text { (DCS) }\end{array}$ & $\begin{array}{l}\text { - } \quad \text { Particle size determination } \\
\text { - } \quad \text { Particle size distribution } \\
\text { - } \quad \text { Suitable for complex matrices } \\
\text { - } \quad \text { Suited to particle sizes of } 5->1000 \mathrm{~nm}\end{array}$ & $\begin{array}{l}\text { - Particles must be more dense that the } \\
\text { liquid they are suspended in } \\
\text { - Low density particles either cannot be } \\
\text { analysed or involve a long analysis } \\
\text { time. }\end{array}$ & $\begin{array}{ll}\text { - } & \text { Proteins } \\
\text { - } & \text { Virus characterisation } \\
\text { - } & \text { Nanotechnology particles } \\
\text { - } & \text { Metal particles }\end{array}$ \\
\hline
\end{tabular}




\begin{tabular}{|c|l|l|l|}
\hline Technique & \multicolumn{1}{|c|}{ Strengths / Benefits } & \multicolumn{1}{c|}{ Limitations } & Used to size, characterise or image \\
\hline & $\bullet \begin{array}{l}\text { Can separate particles of very small } \\
\text { size differences }\end{array}$ & & Cell composition \\
\hline $\begin{array}{c}\text { Particle Induced X-ray } \\
\text { Emission } \\
\text { (PIXE) }\end{array}$ & $\bullet \quad \begin{array}{l}\text { No sample preparation needed } \\
\text { can measure }\end{array}$ & $\begin{array}{l}\text { Sensitivity in the } \mathrm{mg} / \mathrm{L} \text { range } \\
\text { Cannot provide quantitative size } \\
\text { distribution and particle size } \\
\text { A developing technique in single } \\
\text { particle analysis }\end{array}$ & $\bullet \quad$ Elemental particles \\
\hline
\end{tabular}

*EDX - Energy Dispersive X-ray analysis 
Degueldre and Favarger ${ }^{233}$ demonstrated that the ion cloud produced by a single particle in the plasma of an ICP could be detected and measured by the MS. The intensity of the signal produced related to the size of the particle and the frequency of these signals, or 'flashes' as they termed them, related to the concentration of particles in the solution. As outline in section 1.6.3.2 of this thesis, the ICP-MS is operated in time resolved analysis (TRA) mode, with a low flow rate and a much reduced data acquisition time (dwell time) of a few milliseconds. However, as SpICPMS is a new application, the ICP-MS in TRA mode will produce a time scan of peaks over the acquired time representing the signals or flashes only. The software can produce nothing else with this huge amount of raw data, meaning data manipulation has to be conducted offline, after exportation into a suitable software package (such as excel) where numerous, lengthy equations and calculations are semi-manually generated to determine the frequency of particles, particle diameter, size distributions and subsequently mass concentration.

From 2003, a series of papers by Degueldre et $\left.a\right|^{233,271-274}$ outlined several theoretical equations required to measure colloids in solution and in 2011, Laborda et al ${ }^{236}$ also presented the potential of spICP-MS with several theoretical equations and calculations. However, these papers are somewhat lacking in the explanation of the spICP-MS terms used and how data is generated to perform the equations to enable manipulation of the raw data. Fortunately, Pace et $a l^{238}$ developed a practical guide on the fundamentals, including an explanation of terms and outlined the steps needed to generate the data to be inputted into the equations and calculations required to quantitatively count and size particles. For the ease of everyone in the ICP-MS scientific community, the RIKILT research institute, working from within Wageningen University in the Netherlands, developed a spICP-MS procedure ${ }^{275}$ and a spreadsheet single particle calculation tool ${ }^{276}$ for all within the field to use. The spreadsheet is an Excel based document, into which the raw data generated by the ICP-MS for sample analysis is imported. It calculates the larger and more complicated theoretical equations to give you a particle frequency, particle mass concentration and particle size, in addition to a graphical particle size distribution. However, a number of factors 
need to be known and inserted into the spreadsheet such as particle density, an ionic calibration intensity in $\mathrm{cps} /(\mathrm{ug} / \mathrm{L})$, nebulisation efficiency and background threshold. The promising work outlined in Chapter 6, measuring respirable crystalline silica (RCS) by spICP-MS was performed using the RIKILT single particle calculation tool.

The basic principal of spICP-MS is to acquire not more than one particle arriving at the detector in one dwell time. This is called the 'single particle rule'. A dwell time of approximately $3 \mathrm{~ms}$ is common with an acquisition time of $60 \mathrm{~s}$. Therefore, a maximum of 20000 particles can be detected per minute, with the detection of a particle known as a particle event. However, this can result in what is termed a multiple event, where more than one particle is detected at a time. Therefore, a dilution of the particle suspension is required to satisfy the single particle rule and reduce the occurrence of a multiple event. Ideally, RIKILT states there should not be more than 2000 particle events per minute. The background threshold relates to the continuous background baseline signal that a particle has to generate a large enough pulse/peak to be detectable from. A blank sample is run following the same $3 \mathrm{~ms}$ dwell time and $60 \mathrm{~s}$ acquisition time; the background threshold is then calculated as five times the standard deviation of the blank plus the mean. When a blank is analysed, no more than 10 particle events should be above this calculated background threshold.

The nebulisation efficiency is calculated using Equation 1, where; $N n=$ nebulisation efficiency, $q_{p}=$ particle events (number of particles detected above the base line in a minute), $C_{p}=$ Particle number concentration and $V=$ Sample flow rate.

\section{Equation 1}

$$
N n=\frac{q_{p}}{c_{p} V} \times 100
$$

However, the particle event $\left(q_{p}\right)$ and particle number concentration $\left(C_{p}\right)$ can only be calculated using a particle sized CRM. Currently only two commercially available gold nanoparticle CRMs are available, with the $60 \mathrm{~nm}$ (NIST 8013) and 30nm (NIST 8012) being suitable for this application. The CRM is diluted low enough to allow less than 
2000 particle events in a minute above the background threshold and Equations 2 and 3 below are used to determine particle number concentration $\left(C_{p}\right)$ :

Equation 2

$$
M_{p}=\frac{\pi d^{3}}{6} \times p
$$

Where;

$M_{p}=$ particle mass $(\mathrm{g})$

$d=$ particle size $(\mathrm{cm})$

$p=\operatorname{density}(\mathrm{g} / \mathrm{mL})$
Equation 3

$$
C_{p}=\frac{C_{m}}{M_{p}}
$$

Where;

$C_{p}=$ particle number concentration $(\mathrm{mL})$

$C_{m}=$ mass concentration $(\mathrm{g} / \mathrm{mL})$

$M_{p}=$ particle mass $(\mathrm{g})$

For example, the RIKILT procedure suggests a concentration of $50 \mathrm{ng} / \mathrm{L}$ for any CRM as a starting point for these calculations to determine less than 2000 particle events per minute (in this work using the iCAP Q ICP-MS at HSL, $25 \mathrm{ng} / \mathrm{L}$ is a preferable concentration). When performing Equation 2, using the $30 \mathrm{~nm}$ NIST 8012 reference material, and remembering to adjust to the correct units (e.g. $30 \mathrm{~nm}$ to $\mathrm{cm}$ ), the $M_{p}$ is $2.7 \times 10^{-16} \mathrm{~g}$. Inserting the acquired $M_{p}$ value and other data into Equation 3, adjusting to the correct units (e.g. $25 \mathrm{ng} / \mathrm{L}$ to $\mathrm{g} / \mathrm{mL}$ ), the $C_{p}$ is $88273 \mathrm{~mL}$. Including the number of particle events counted above the background all the information needed for Equation 1 has been acquired. An ionic gold calibration is performed and the ICP-MS response to the ion standard of the element in $\mathrm{cps} /(\mu \mathrm{g} / \mathrm{L})$ is obtained and inserted into the spreadsheet. When analysing particles of which a sized particle CRM is not available, the procedure outlined above is still followed. However, an ionic calibration of the element to be analysed is also performed with the ICP-MS response in $\mathrm{cps} /(\mu \mathrm{g} / \mathrm{L})$ and its density being incorporated into the RIKILT spreadsheet, in addition to those of gold. Figure 17 shows the table included in the RIKILT calculation tool; the orange boxes indicate the information that the user must know and insert to enable the spreadsheet to perform the calculations. Using these calculations the RIKILT calculation tool has measured the $25 \mathrm{ng} / \mathrm{L} 30 \mathrm{~nm}$ gold CRM as $23 \mathrm{ng} / \mathrm{L}$ and $29 \mathrm{~nm}$ (shown in the yellow box) and the graphical particle size distribution is displayed below. Figure 18 shows the section of the RIKILT table for samples that the particle mass and diameter are not known. The sample demonstrated in Figure 18 is of an HSL in-house RCS reference 
material at an approximate concentration of $50 \mu \mathrm{g} / \mathrm{L}$. The orange boxes indicate the information that the user must know and insert to enable the spreadsheet to perform the calculations as the element of interest is now silica rather than gold. The spreadsheet has calculated that the 132 particles of silica have been detected with an average particle size of $401 \mathrm{~nm}$.

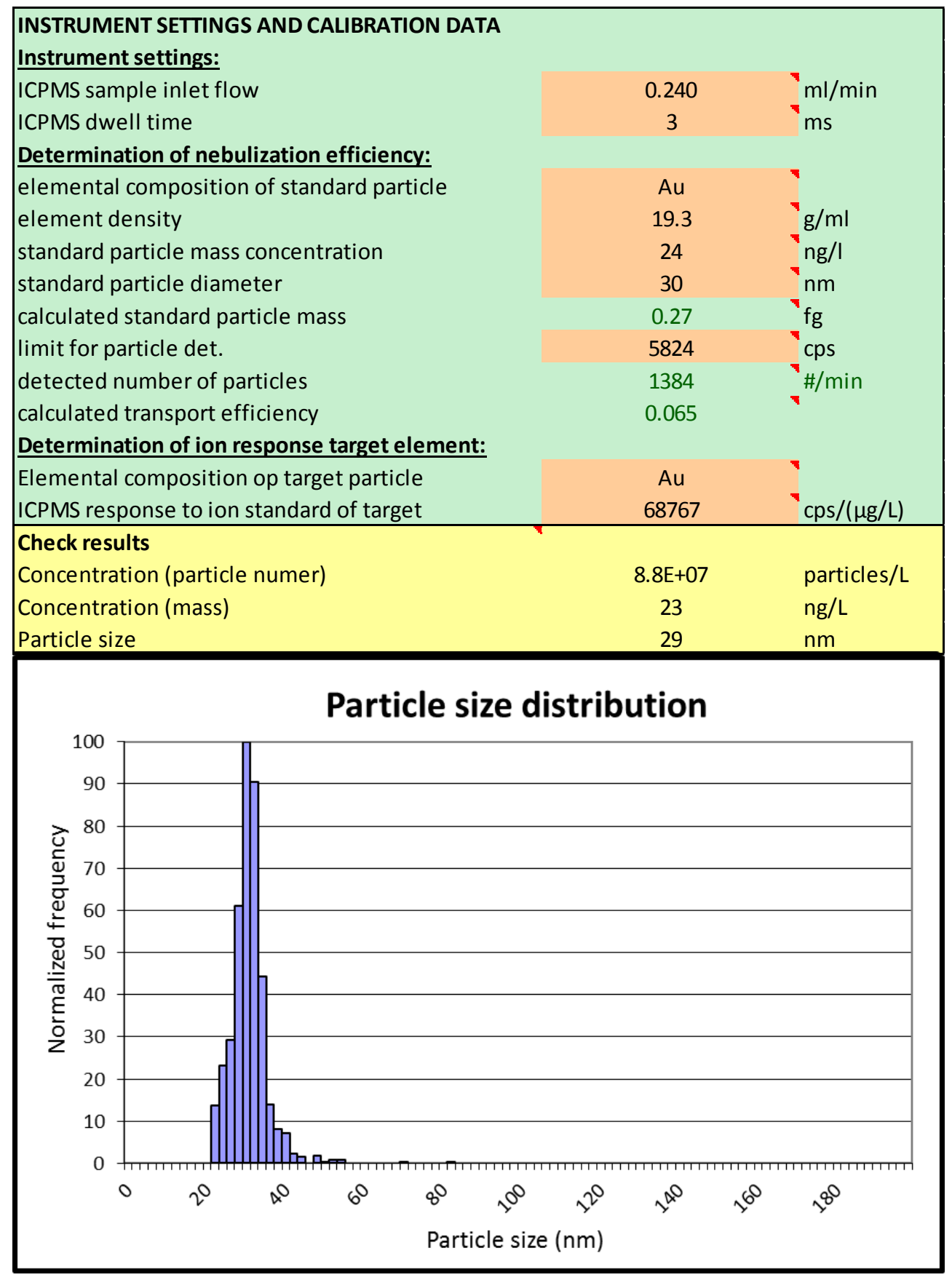

Figure 17 A section of the RIKILT calculation tool spreadsheet showing the known criteria and result of the calculated nebulisation efficiency using a gold ionic calibration and $30 \mathrm{~nm}$ gold NIST CRM. 


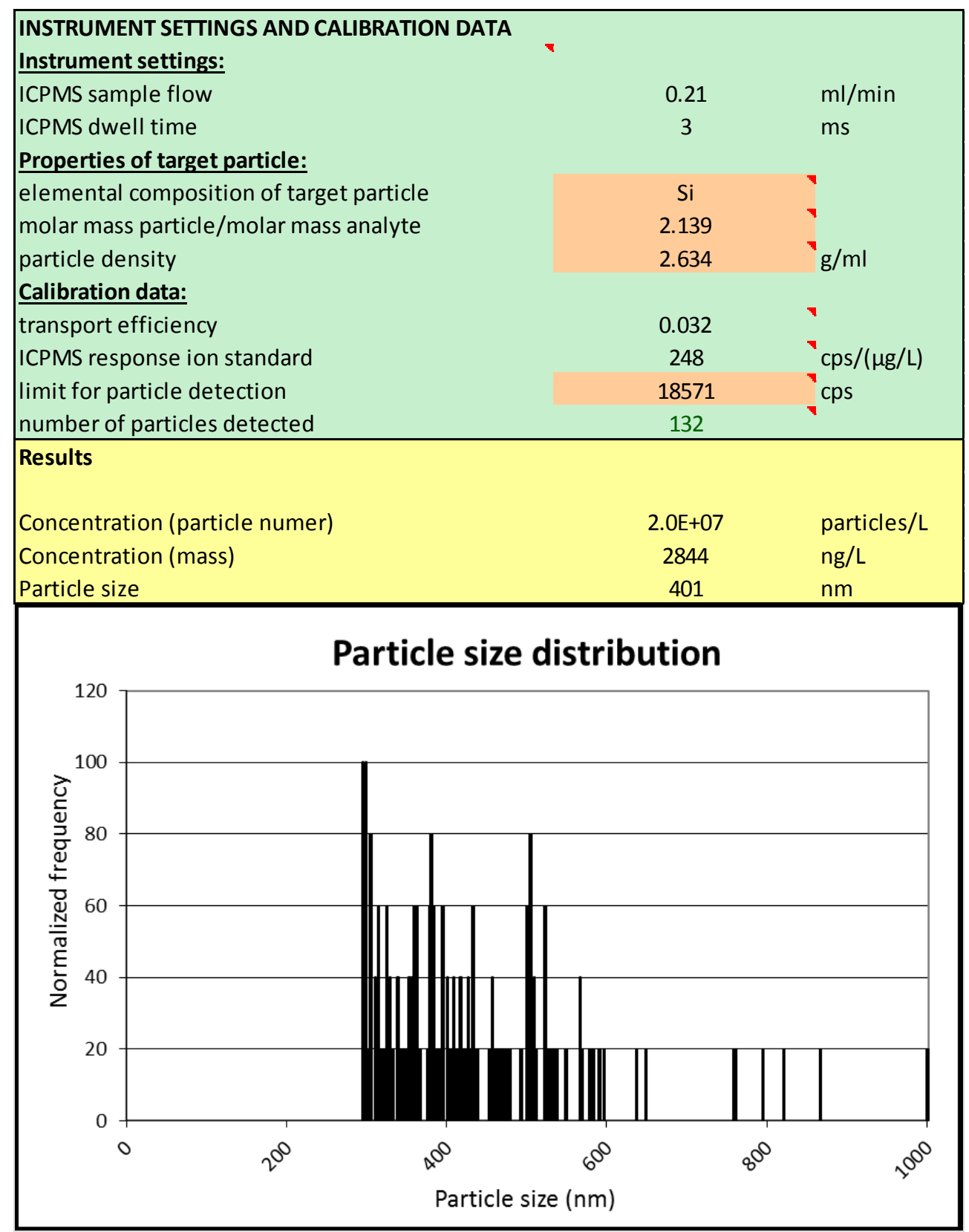

Figure 18 A section of the RIKILT calculation tool spreadsheet showing the known criteria and result from an RCS in-house (A9950) reference material approximately $50 \mu \mathrm{g} / \mathrm{L}$

\subsubsection{Advancements in Single Particle Analysis}

Advances in ICP-MS technology since beginning this investigation to measure single particles of silica in EBC samples have been the introduction of ICP-MS single particle software. In 2015, Thermo Scientific launched an optional single particle software plugin as an upgrade available to purchase for the ICAPQ ICP-MS, called npQuant. The 
other ICP-MS manufacturers also each have their own single particle software for their instruments. All of these software packages and the associated calculations are based on the RIKILT procedure and spreadsheet, with each being able to calculate the nebulisation efficiency. However, for npQuant, manual calculations of $M_{p}$ and $C_{p}$ using Equations 2 and 3 , are still required by the user.

Another advancement has been the introduction of a triple quadrupole (QQQ) ICP-MS by Agilent Technologies (Cheadle, UK). The QQQ ICP-MS adds an MS/MS mode for interference removal. It consists of two quadrupole mass filters with the collision/reaction cell quadrupole situated in between. This allows for two options of analysis using the two mass filters. The first option is mass shift analysis, where the mass of the element of interest is applied to the first mass filter quadrupole and the second mass filter quadrupole is the sum of the mass plus the mass of the reaction cell gas, which enables the elimination of polyatomic interferences. The second option, which is employed for silica analysis, is on-mass selection analysis, where both mass filter quadrupoles are set to $\mathrm{m} / \mathrm{z}$ 28, as shown in Figure 19.

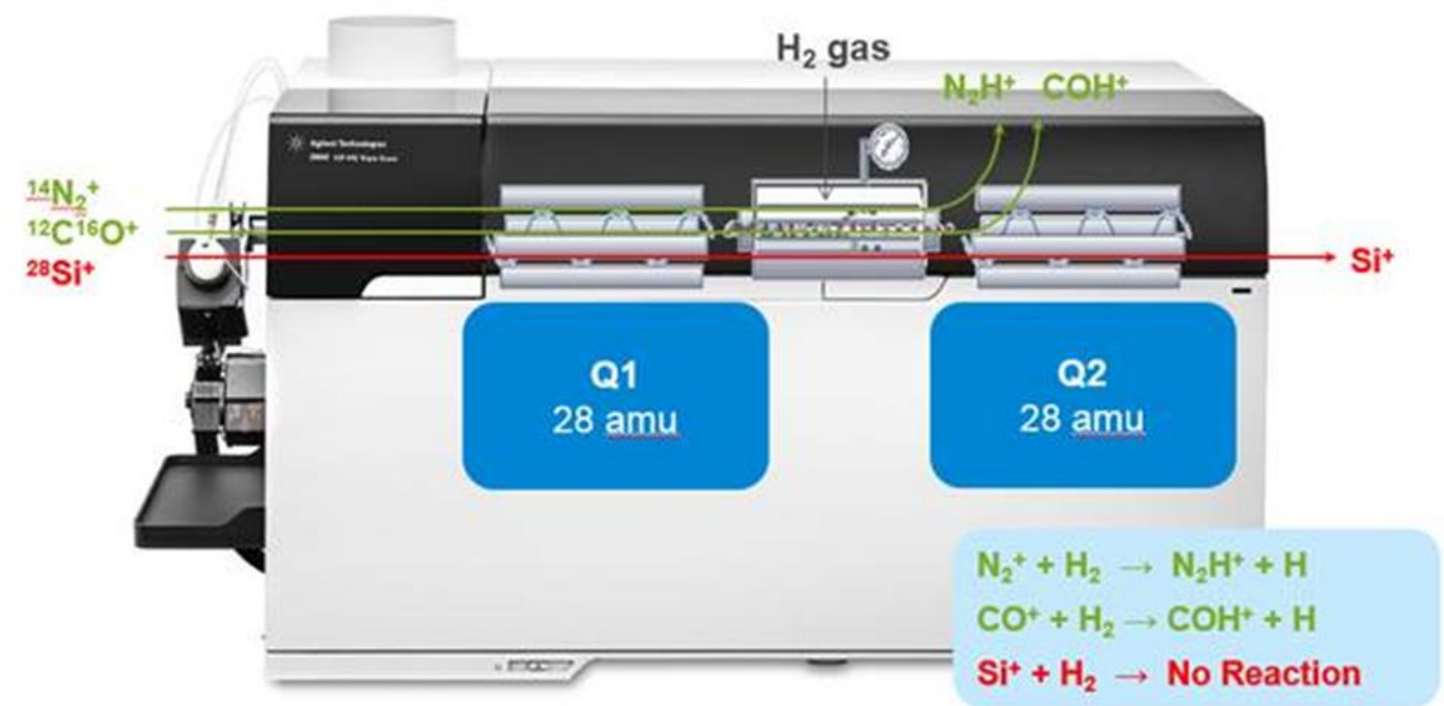

Figure 19 A QQQ ICP-MS/MS consisting of two mass filters and a collision/reaction cell situated in between, demonstrating its efficiency at removing nearly all polyatomic interferences, by applying $\mathrm{m} / \mathrm{z} 28$ to both mass filters. (C Agilent Technologies, Inc. 2017. Image kindly supplied by Agilent Technologies, Inc. 
As demonstrated in Figure 19, the polyatomic interferences $\left({ }^{14} \mathrm{~N}_{2}{ }^{+}\right.$and ${ }^{12} \mathrm{C}^{16} \mathrm{O}^{+}$and ${ }^{28} \mathrm{Si}$ ions pass through the first mass filter set at $\mathrm{m} / \mathrm{z} 28$ and subsequently enter the collision/reaction cell. The cell employs hydrogen as a reaction gas to react with the interfering ions to form larger polyatomic ions $\left(\mathrm{N}_{2} \mathrm{H}^{+}\right.$and $\left.\mathrm{COH}^{+}\right)$which are now discarded as they pass through the second mass filter also set to $\mathrm{m} / \mathrm{z} 28$, only allowing ${ }^{28} \mathrm{Si}$ ions to pass to the detector.

The QQQ ICP-MS has greatly enhanced sensitivity and has a reduced limit of detection (LOD) compared to single quadrupole ICP-MS. This ability to further reduce polyatomic interferences allows for a lower background threshold when analysing silica particles which in turn enables smaller particle sizes to be measured. In Chapter 6 of this thesis, the particle size LOD for crystalline silica is estimated to be approximately $300 \mathrm{~nm}$ (depending on daily sensitivity and the background signal of $\mathrm{m} / \mathrm{z} 28$ ). Other studies have reported a particle size LOD for colloidal silica particles of $200 \mathrm{~nm},{ }^{235} 300 \mathrm{~nm},{ }^{277}$ $403 \mathrm{~nm}^{278}$ and $572 \mathrm{~nm},{ }^{279}$ whilst studies using a QQQ ICP-MS have reported particle size LODs for colloidal silica down to $20 \mathrm{~nm} \cdot{ }^{280-282} \mathrm{In}$ addition the second generation QQQ ICP-MS from Agilent Technologies incorporates a re-engineered argon gas flow path, which helps to further reduce background contamination of silicon and sulphur. It also has a more powerful and faster detector, allowing a dwell time as low as 0.1 $\mathrm{ms}^{262}$ (the first generation Agilent Technologies QQQ ICP-MS and all single quadrupole ICP-MS detectors only allow a dwell time as low as $3 \mathrm{~ms}$ ). Thermo Scientific unveiled their first QQQ ICP-MS in February 2017 at the European Winter Conference on Plasma Spectrochemistry.

Further applications include the hyphenation of spICP-MS with front-end separation techniques such as flow-field flow fractionation $(\mathrm{A} 4 \mathrm{~F})^{283}$ or hydrodynamic chromatography (HDC). ${ }^{283}$

The use of different reaction/collision cell gases is an alternative option to increasing sensitivity and reducing background noise. The ICAP Q ICP-MS at the HSL uses helium gas as the standard Thermo Scientific recommended cell gas. Other studies have reported using helium, ${ }^{277} 7 \%$ hydrogen in helium ${ }^{279}$ and ammonia, ${ }^{278,284}$ however hydrogen also seems to be very efficient. ${ }^{285}$ In addition, the use of non-quartz based 
glassware for the torch, spray chamber and injector of the sample introduction setup in the form of pfa (perfluoroalkoxy), ceramic, platinum and sapphire have all been reported to help reduce the silicon background. ${ }^{282,284}$ All of these aspects and alternative approaches will need further research at HSL to help improve and develop a RCS spICP-MS method.

\subsubsection{Current spICP-MS Limitations}

Although the development of single particle analysis is extremely exciting, it still has a number of limitations that are currently holding back progress, as well as reducing the accuracy and precision of the data obtained.

The primary limitation is the lack of sized particle CRMs. At present, the main option to calculate the nebulisation efficiency is by using gold particles. This nebulisation efficiency value is crucial, as it is used to calculate particle frequency, particle mass concentration and particle size distribution. This means that the nebulisation efficiency of a gold particle is used to calculate the particle frequency, mass and distribution of other elemental particles. However, it cannot be assumed that the nebulisation efficiency of gold particles would match those of zinc, RCS or titanium particles, for example.

Another limitation is the reliance on the spICP-MS software to calibrate using an ionic standard rather than calibrating using the single particles themselves. A calibration of one single particle size at different mass concentrations could be used; however, it has not been investigated whether using one single particle size would be accurate when measuring particles across the $\mathrm{nm}$ size range. Several calibrations of different sized particles could be employed, for example three separate calibrations of a small, medium and large sized particle dependent on the expected particle sizes in the samples to be analysed, of which an average could be taken. Again, this is yet to be investigated. However, it is known that the signal intensity of different sized particles at the same mass concentration is not linear. As previously stated, the intensity of the signal produced by a single particle is related to its size, however it is also proportional 
to the cube of the particle diameter. So for example, the intensity signal produced by the $30 \mathrm{~nm}$ NIST gold CRM is not half that of the $60 \mathrm{~nm}$ NIST gold CRM but 1/8 and, if a $15 \mathrm{~nm}$ NIST gold CRM existed, it would produce an intensity signal 1/64 that of the 60 $\mathrm{nm}$ NIST gold CRM. Therefore, a calibration of different particle sizes would need to consider this.

Regarding RCS, the greatest limitation is the shape of the actual RCS particles. The calculations used to determine particle size for spICP-MS in addition to other techniques such as particle/nano tracking analysis are all based on the assumption that the particles are spherical. The TEM micrographs obtained at HSL of two different RCS particles shown in Figure $\mathbf{2 0}$ demonstrate that not only are RCS particles non-spherical but they are irregular. The two RCS silica particles, identified and sized by TEM, are 5 $\mu \mathrm{m}(5000 \mathrm{~nm})$ and $0.5 \mu \mathrm{m}(500 \mathrm{~nm})$ and were observed in the EBC sample of a quarry worker taking part in the work outlined in Chapter 6 of this thesis. However the fact that there is currently no biological monitoring available to assess occupational exposure to RCS and using spICP-MS can easily and quickly detect and count the frequency of RCS particles in an EBC sample far outweighs the fact that the particle sizing is likely to be inaccurate.

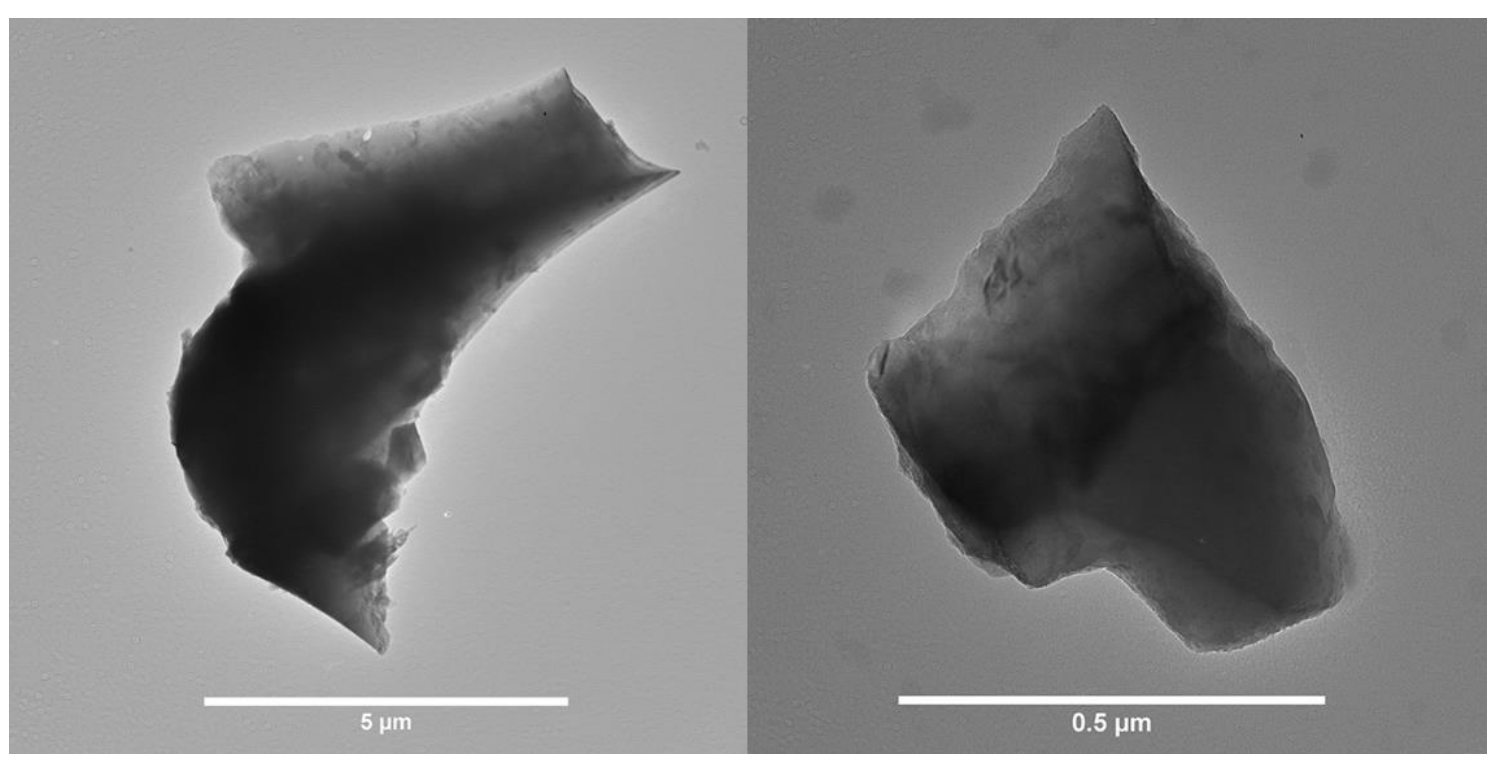

Figure 20 Micrographs of two different respirable crystalline silica particles, demonstrating the irregularity in shape using a Tecnai G2 Spirit 120kV TEM with attached X-MAX energy dispersive spectroscopy system. 
As spICP-MS applications grow in popularity and more varied sample types are investigated, the measurement of multiple particle sizes in a single sample is going to be challenging. An individual, occupationally exposed to RCS, will inhale all sizes of crystalline silica across the respirable size range. However, as demonstrated in Figure 18 , the results are expressed as an average particle size and mass. A strength of the ICAP Q spICP-MS software is that it provides a choice, to build a method to either calculate all the particles as an average or to measure individual sizes of particles. In the software a minimum and maximum threshold is inserted which specifies the minimum and maximum peak height of the intensity signals. Therefore, different sets of minimum and maximum thresholds can be inserted to correspond to the different intensity signal peak heights from different $\mathrm{nm}$ sizes of particles. This is easily performed with two distinct sized particles such as the $30 \mathrm{~nm}$ and $60 \mathrm{~nm}$ gold NIST CRMs. However, it is considerably more challenging with an EBC sample, which may contain many different sized particles across the $\mathrm{nm}$ size range, where particles can vary in size from $100 \mathrm{~nm}$ up to $1000 \mathrm{~nm}$.

Instrument cleanliness and contamination has also proven to be a limitation at the HSL for the analysis of silica and gold particles. The iCAP Q ICP-MS is no longer used for sample analysis outside of this work here if the reagents needed for the application contain high concentrations of gold or silica, in addition to the samples themselves (for example, elemental quantification of air monitoring samples on glass fibre filters) to maintain the low background threshold required for spICP-MS analysis.

\subsection{Silica Particles}

The reporting of spICP-MS results for RCS particles will need thoughtful consideration, for example, how to convert the particle frequency and the many sizes of crystalline silica into a result that is understandable and transferable to the entire community from spICP-MS users, occupational hygienists and GPs to finally the employers and employees. For crystalline silica to be classed as RCS it has to be less than $10 \mu \mathrm{m}$ in size to enter the alveoli regions of the lungs where it is able to cause damage and induce lung diseases such as silicosis. Although the link between occupational exposure to RCS 
and its related health effects is well documented very little is known about the effect of particle size and the development of disease. ${ }^{286,287}$ It is suggested that the surface chemistry of particles allows for increased reactivity within cells ${ }^{288}$ and so the smaller the particle the larger the surface area to volume ratio resulting in higher reactivity per gram of a chemical. ${ }^{262}$ However, as RCS particles are not spherical, a more irregular shaped particle may have a larger surface area to volume ratio than a smaller less irregular shaped particle. An in vitro study looking at the uptake and biological activity of different sized colloidal silica particles in macrophage cells from mice found that 850 $\mathrm{nm}$ particles appeared more biologically active than 150, 250 and $500 \mathrm{~nm}$ particles, however the smallest sized particles in the study of $150 \mathrm{~nm}$ had the greatest uptake. ${ }^{289}$ Wiessner et $a l^{290}$ demonstrated that larger crystalline silica particles had a greater in vivo inflammatory response than smaller particles; the same was also demonstrated by Kajiwara et al. ${ }^{291}$ However, a more recent study involving crystalline silica particles as small as $300 \mathrm{~nm}$ found the smallest particles generated more mitochondrial reactive oxygen species than larger particles. ${ }^{286}$ It has also been suggested that smaller particles of less than $100 \mathrm{~nm}$ in size are more easily cleared from the lungs and into the circulatory system by mucociliary clearance, whereas the larger particles remain, causing persistent inflammation and cell death. ${ }^{292}$ With this in mind, a more simplistic approach may be to generate a result consisting only of the number of RCS particles detected in EBC in comparison to the $95^{\text {th }}$ percentile of RCS particles found in a background population. This could be increased from the frequency to also include the minimum and maximum size found, along with where the greatest percentage of particles was found within that range. Because of the irregularity in shape, the most obvious suggestion would be to no longer focus on trying to express RCS as a nm size, but to maybe present it as a density in the form of the particle mass per unit volume.

As already stated, the occupational exposure assessment of crystalline silica using biological monitoring is not currently possible, though EBC is demonstrating the potential to resolve this problem. However, a small handful of studies have already emerged, investigating biomarkers of oxidative stress for the early diagnosis of silicosis and the primary effects of silica exposure. A study by Sauni et $a l^{293}$ found increased nitric oxide levels in the EBC of workers occupationally exposed for more than 20 years 
to silica in comparison to an unexposed control group. The authors postulate that exposure to crystalline silica induces an inflammatory response prior to the formation of silicosis and that the measurement of nitric oxide could be used to identify individuals at risk of developing silicosis. Aldehydes are a known oxidation product used for the quantification of oxidative stress, ${ }^{294}$ with Sakhvidi et al investigating malondialdehyde (MDA) in tile and ceramic production workers employed for longer than 2 years compared with a control group; however no significant difference between EBC MDA concentrations was found between the two groups. The studies by Sauni et $a l^{293}$ and Sakhvidi et $a l^{294}$ both eliminated volunteers with pre-existing pulmonary conditions such as asthma and respiratory or lung diseases. In contrast, other studies have purposefully focused on silica exposed workers with occupational lung diseases. The first study to report markers of oxidative stress in EBC samples of silicosis patients was by Pelclova et $\mathrm{al}^{48}$ in 2007 . The study compared oxidative stress biomarkers 8-isoprostane and four leukotrienes in silicosis patients with a control group. They reported elevated EBC levels of both 8-isoprostane and one leukotriene (D4) only in silicosis patients compared to controls. ${ }^{48}$ In 2011, Pelclova et al ${ }^{51}$ compared levels of 8-isoprostane, in addition to aldehydes MDA and hydroxynonenal (HNE), in patients with varying degrees of asbestosis and silicosis against a control group. The study reported elevated levels of 8-isoprostane and HNE in EBC samples of both asbestosis and silicosis patients in comparison to the controls, but not for MDA. ${ }^{51}$ Another study, comparing four different leukotrienes in EBC samples, in addition to the lung function of patients with varying degrees of asbestosis or silicosis, was published by the same research group the following year. ${ }^{50}$ Although lung function was significantly impaired in both silicosis and asbestosis patients the amount of EBC collected did not differ between the two groups or when compared against the control group. ${ }^{50}$ The study reported elevated levels of two leukotrienes (D4 and E4) in EBC samples of both sets of patients compared to the control group, along with elevated leukotrienes (C4 and cysteinyl) in EBC samples of asbestos patients only. ${ }^{50}$ Additionally, increased levels of leukotrienes correlated to the severity of asbestosis and a significant correlation was found between leukotriene concentration and total lung capacity of asbestosis patients. However, no correlation between leukotriene levels 
and disease severity or lung function could be found for the silicosis patients. ${ }^{50} \mathrm{~A}$ similar study comparing asbestosis and silicosis patients reported a simultaneous method to analyse 8-isoprostane in addition to MDA and HNE. ${ }^{47}$ Unlike the Sakhvidi ${ }^{294}$ and Pelclova ${ }^{51}$ studies, MDA levels in EBC were shown to be significantly elevated in comparison to the control group in addition to both HNE and 8-isoprostane. ${ }^{47}$ However, in the Sakhvidi study the volunteers did not have silicosis. ${ }^{294}$

The work outlined in Chapter 6 is the only study to report silica particles in EBC samples of occupationally exposed workers and controls. However, one other study has attempted to do the same. This very recent study, published online at the end of January 2017 by Sauvain et $a l_{,}^{295}$ reports the particle number concentration and size distribution of particles (of unknown elemental composition) in EBC samples of workers exposed to soapstone or quartz dust using nano tracking analysis (NTA). However, unlike spICP-MS, NTA cannot determine the precise nature of the particles nor differentiate between elemental compositions of the different particles. Although environmental monitoring of personal air samplers confirmed the respirable dust fractions collected gravimetrically contained silica (confirmed by x-ray diffraction, but not specifying amorphous or crystalline silica), the SEM analysis of the EBC samples identified particles containing sodium, potassium, chlorine, sulphur, calcium, magnesium, aluminium, iron, titanium and silicon. Therefore, the particles counted and the particle size distributions produced would be inclusive of all these particles of different composition. However, it was reported that particle size variability was significantly higher in crystal quartz workers compared to soapstone workers and the control group, in addition to the number of particles detected also being significantly higher. The particle sizes found in all volunteer EBC samples, ranged from $45 \mathrm{~nm}$ up to $290 \mathrm{~nm}$ using NTA. However, silica particles of approximately $1000 \mathrm{~nm}$ were confirmed by SEM analysis that were not detected by NTA, indicating that NTA may not be a suitable technique for RCS identification. 


\subsection{The Potential of other Elemental Single Particle Analysis}

The method of spICP-MS allows for the analysis of particle suspensions in a liquid sample, in particular metal and metalloid oxide particles. Although considerable progress has been made, this is still an emerging technique with the majority of publications involving the development and optimisation of the technique using commercially available particles as pristine particle test cases rather than the analysis of particles in authentic samples and applications. However, a small handful of studies is beginning to emerge consisting of authentic spICP-MS applications which include: an attempt to measure silver nanoparticles in blood samples of burns victims treated with silver nanoparticle dressings; ${ }^{296}$ the measurement of arsenic particles from environmental sample leachates from mine waste; ${ }^{297}$ detection of zinc oxide and cerium oxide nanoparticles in drinking water treatment facilities. ${ }^{298}$

It may be possible that spICP-MS could be used to detect chromium particulate in EBC samples. For example, specifically for workers who potentially inhale aerosolised chromium particulates such as welders and grinders as opposed to chrome platers who inhale chromium vapour. However, exposure to soluble chromium and fumes creating an elevated background signal within the samples may be of concern for spICP-MS analysis.

The measurement of various metal and metalloid particulates in biological samples by spICP-MS is a possible future development for spICP-MS.

\subsection{Conclusion}

The aims of this PhD study were to develop new analytical speciation methods and to evaluate exhaled breath condensate, for its potential as a new biological matrix for exposure assessment. This has been achieved to varying degrees with arsenic, chromium and silica. The developed arsenic speciation method is now utilised routinely at HSL and will continue to be so. For chromium, a speciation method has been developed and both $\mathrm{Cr}(\mathrm{III})$ and $\mathrm{Cr}(\mathrm{VI})$ have been detected in $\mathrm{EBC}$ samples of workers occupationally exposed to $\mathrm{Cr}(\mathrm{VI})$. Future work is planned to further 
understand $\mathrm{Cr}(\mathrm{VI})$ inhalation exposures and its correlation to $\mathrm{EBC}$ measurements. For silica, the work is less developed, however the analytical technique shows promise. RCS particles in EBC samples of workers occupationally exposed to crystalline silica have been detected and the technique of spICP-MS will be explored further.

The better understanding, detection and measurement of arsenic species in urine, chromium species in EBC and RCS particles in EBC should therefore mean reduction in worker exposure will be possible using the techniques developed. 


\section{Chapter 8.}

Publications, Oral Presentations and Poster Presentations. 


\subsection{Publications}

1. E. Leese, J.F. Staff, V.A. Carolan and J. Morton. Exhaled breath condensate: A novel matrix for biological monitoring to assess occupational exposure to respirable crystalline silica. Ann. Work. Exp. Health. (formerly Ann. Occup. Hyg.), 2017, 61(7): 902 - 906. doi: 10.1093/annweh/wxx047.

2. E. Leese, M. Clench, Morton, J. P.H.E. Gardiner and V.A. Carolan. The investigation of unexpected arsenic compounds observed in routine biological monitoring urinary speciation analysis. Toxics. 2017, 5(2): doi:10.3390/toxics5020012

3. E. Leese, J. Morton, P.H.E. Gardiner and V.A. Carolan. The simultaneous detection of trivalent and hexavalent chromium in exhaled breath condensate: A feasibility study comparing workers and controls. Int. J. Hyg. Environ. Health., 2017, 220: 415423.

4. E. Leese, J. Morton. P.H.E. Gardiner and V.A. Carolan. Development of a method for the simultaneous detection of $\mathrm{Cr}(\mathrm{III})$ and $\mathrm{Cr}(\mathrm{VI})$ in exhaled breath condensate samples using HLC-ICP-MS. J. Anal. At. Spectrom., 2016, 31(4): 924-933.

5. E. Leese, J. Morton, E. Tan, P.H.E. Gardiner and V.A. Carolan. MLC-ICP-MS determination of unexposed UK urinary arsenic speciation reference values. J. Anal. Toxicol., 2014, 38(1): 24-30.

\subsection{Oral Presentations}

1. Supra-Regional Assay Service/Association of Clinical Biochemistry: Trace Elements Meeting, $20^{\text {th }}$ October 2016, York, UK. Exhaled breath condensate: A new biological matrix to measure and detect trivalent and hexavalent chromium in occupationally exposed workers

2. The $18^{\text {th }}$ Biennial National Atomic Spectroscopy Symposium, $4^{\text {th }}-6^{\text {th }}$ July 2016 , John Moore's University, Liverpool, UK. Exhaled breath condensate: A new biological matrix to measure and detect trivalent and hexavalent chromium in occupationally exposed workers. 
3. Thermo UK ICP-MS User Meeting, $16^{\text {th }}$ June 2016, Jodrell Bank Discovery Centre, Manchester, UK. Exhaled breath condensate: Silica single particles and their effect on health.

4. Surface Engineering Association Northern Finishers Meeting, $10^{\text {th }}$ May 2016. Leeds, UK. Exhaled breath condensate measurement of hexavalent and trivalent chromium in occupationally exposed workers.

5. The $4^{\text {th }}$ UK and Ireland Exposure Science Meeting, $20^{\text {th }}$ April 2016, Health \& Safety Laboratory, Buxton, UK. Exhaled breath condensate: A new biological matrix to measure and detect trivalent and hexavalent chromium in occupationally exposed workers.

6. The Surface Engineering Association Annual Industry Conference, $16^{\text {th }}$ October 2015, Stratford Upon Avon, UK. Exhaled breath condensate: A new biological matrix for hexavalent and trivalent chromium.

7. HSE Workshop, $30^{\text {th }}$ July 2015, Health \& Safety Laboratory, Buxton, UK. Exhaled breath condensate: A new biological matrix.

8. Thermo Ion Chromatography and Trace Elements User Meeting, $15^{\text {th }}$ July 2014, Altrincham, UK. Arsenic and chromium speciation methods at the Health \& Safety Laboratory.

9. The $9^{\text {th }}$ International Symposium on Biological Monitoring, $9^{\text {th }}-11^{\text {th }}$ September 2014, Manchester, UK. Arsenic speciation in urine using $\mu$ LC-ICP-MS for routine biological monitoring.

10. Thermo Speciation Analysis Meeting, $17^{\text {th }}$ November 2011, Health \& Safety Laboratory, Buxton, UK. Arsenic speciation by $\mu$ LC-ICP-MS.

\subsection{Poster Presentations}

1. The $3^{\text {rd }}$ UK and Ireland Exposure Science Meeting, $24^{\text {th }}$ April 2015, London, UK. Investigation of arsenic peaks observed in urine from interesting forensic and occupational cases.

2. The $3^{\text {rd }}$ UK and Ireland Exposure Science Meeting, $24^{\text {th }}$ April 2015, London, UK. Single particle ICP-MS of silica in exhaled breath condensate of UK workers. 
3. The $3^{\text {rd }}$ UK and Ireland Exposure Science Meeting, $24^{\text {th }}$ April 2015, London, UK. Speciation of trivalent and hexavalent chromium by $\mu$ LC-ICP-MS in exhaled breath condensate samples: initial results.

4. European Winter Conference on Plasma Spectrochemistry, $22^{\text {nd }}-25^{\text {th }}$ February 2015, Munster, Germany. Investigation of arsenic peaks observed in urine from interesting forensic and occupational cases.

5. European Winter Conference on Plasma Spectrochemistry, $22^{\text {nd }}-25^{\text {th }}$ February 2015, Munster, Germany. Single particle ICP-MS of silica in exhaled breath condensate of UK workers.

6. European Winter Conference on Plasma Spectrochemistry, $22^{\text {nd }}-25^{\text {th }}$ February 2015, Munster, Germany. Speciation of trivalent and hexavalent chromium by $\mu L C$ ICP-MS in exhaled breath condensate samples: initial results.

7. The $17^{\text {th }}$ Biennial National Atomic Spectroscopy Symposium, $31^{\text {st }}$ August $-4^{\text {th }}$ September 2014, Aberdeen University, Scotland. Investigation of arsenic peaks observed in urine from interesting forensic and occupational cases.

8. The $17^{\text {th }}$ Biennial National Atomic Spectroscopy Symposium, $31^{\text {st }}$ August $-4^{\text {th }}$ September 2014, Aberdeen University, Scotland. Speciation of trivalent and hexavalent chromium by $\mu$ LC-ICP-MS in exhaled breath condensate samples: Method development.

9. The Society of Occupational Medicine Annual Scientific Meeting. $2^{\text {nd }}$ July 2014. Establishing background levels for five arsenic species in urine sample in a UK population.

10. The $2^{\text {nd }}$ UK and Ireland Exposure Science Meeting, $4^{\text {th }}$ March 2014, Manchester, UK. Establishing background levels for five arsenic species in urine sample in a UK population.

11. European Winter Conference on Plasma Spectrochemistry, $10^{\text {th }}-15^{\text {th }}$ February 2013, Krakow, Poland. Arsenic speciation in urine using $\mu$ LC-ICP-MS for routine biological monitoring.

12. BMRC Christmas Poster Session, $16^{\text {th }}$ December 2011, Sheffield Hallam University, Sheffield, UK. Arsenic speciation in urine using $\mu$ LC-ICP-MS for routine biological monitoring. 


\section{$\underline{\text { References }}$}

1 A. Spence, Health \& Safety at Work: Summary statistics for Great Britain 2016, http://www.hse.gov.uk/statistics/, (accessed April 2017).

2 HSE, Information about Health \& Safety at Work, http://www.hse.gov.uk/index.htm, (accessed November 2016).

3 HSL, About HSL, http://www.hsl.gov.uk/about-hsl, (accessed November 2016).

$4 \quad$ HSL, What we do: Worker Protection, http://www.hsl.gov.uk/what-wedo/worker-protection, (accessed November 2016).

5 HSE, Biological Monitoring in the Workplace: A guide to its Practical Application to Chemical Exposure, HSE Books, 2nd edn, 1997.

6 A. Aitio, A. Bernard, B. A. Fowler and G. F. Nordberg, in Handbook on the Toxicology of Metals, ed. G. F. Nordberg, B. A. Fowler, M. Nordberg and L. T. Friberg, Elsevier, London, 3 edn, 2007, ch. 4, pp. 65 - 78.

7 HSE, Control of substances hazardous to health regulations 2002, HSE, 6th edn, 2013.

8 HSE, Control of Lead at Work, HSE Books, 3rd edn, 2002.

$9 \mathrm{HSL}$, Guidance on Laboratory Techniques in Occupational Medicine, HSE Books, 12th edn, 2013.

10 J. Cocker, K. Jones, J. Morton and H. J. Mason, Int. J. Hyg. Environ. Health, 2007, 210, 383-386.

11 IUPAC, Compendium of Chemical Terminology, 2nd edn. (the "Gold Book"), Blackwell Scientific Publications, Oxford, last updated February 2014, http://goldbook.iupac.org, (accessed January 2017).

12 C. G. Elinder, L. T. Friberg, T. Kjellstrom, G. F. Nordberg and G. Oberdoerster, Biological Monitoring of Metals, World Health Organisation, Geneva, 1994. http://www.who.int/iris/handle/10665/62052.

13 T. Brorson, G. Skarping and J. Nielsen, Int. Arch. Occup. Environ. Health., 1990, 62, 385-389.

14 J. M. Christensen, Sci. Total Environ., 1995, 166, 89-135.

15 S. M. Cohen, L. L. Arnold, M. Eldan, A. S. Lewis and B. D. Beck, Crit. Rev. Toxicol., 2006, 36, 99-133.

16 ATSDR, Toxicological profile for chromium, Department of Health and Human Services, Atlanta, 2012.

http://www.atsdr.cdc.gov/ToxProfiles/tp.asp?id=62\&tid=17, (accessed May 2017).

17 WHO, Environmental Health Criteria 61: Chromium, World Health Organisation, Geneva, 1988. http://www.inchem.org/documents/ehc/ehc/ehc61.htm, (accessed May 2017). 
CDC, National Biomonitoring Program: Biomonitoring Summary: Mercury, https://www.cdc.gov/biomonitoring/Mercury_BiomonitoringSummary.html, (accessed May 2017).

19 S. Pruksa, A. Siripinyanond, J. J. Powell and R. Jugdaohsingh, Nutr. Metab., 2014, 11.

20 G. F. Nordberg, B. A. Fowler, M. Nordberg and L. T. Friberg, Handbook on the Toxicology of Metals, Elsevier Academic Press, London, 3rd edn, 2007.

21 J. Cocker, H. J. Mason, N. D. Warren and R. J. Cotton, Occup. Med., 2011, 61, 349-353.

22 ACGIH, TLVs and BEIs: Threshold Limit values for Chemical Substances and Physical Agents, Signature Publications, USA, 2017.

23 DFG, List of MAK and BAT values 2012, Wiley - VCH, Germany, 2012.

24 HSE, EH40/2005 Workplace Exposure Limits, http://www.hse.gov.uk/pubns/books/eh40.htm).(accessed May 2017).

25 J. Cocker, Toxicol. Lett., 2014, 231, 122-125.

26 J. Morton, E. Tan, E. Leese and J. Cocker, Toxicol. Lett., 2014, 231, 179-193.

27 IUPAC Periodic Table of the Elements, https://iupac.org/what-we-do/periodictable-of-elements/ (accessed January 2017).

28 P. Apostoli, J. D. Cornelis, P. Hoet, D. Lison and D. Templeton, Environmental Health Criteria 234: Elemental Speciation in Human Health Risk Assessment, World Health Organisation, Geneva, http://apps.who.int/iris/handle/10665/43442 (accessed May 2017).

29 G. F. Nordberg, B. A. Fowler, M. Nordberg and L. T. Friberg, in Handbook on the Toxicology of Metals, ed. G. F. Nordberg, B. A. fowler, M. Nordberg and L. T. Friberg, Elsevier Academic Press, London, 3rd edn, 2007, ch. 1, pp. 1-10.

30 Q. Ke, M. Costa and G. Kazantzis, in Handbook on the Toxicology of Metals, ed. G. F. Nordberg, B. A. Fowler, M. Nordberg and L. T. Friberg, Elsevier Academic Press, London, 3rd edn, 2007, ch. 10, pp. 177-196.

31 IARC, Agents classified by the IARC monographs, volumes 1-117, http://monographs.iarc.fr/ENG/Classification/latest_classif.php, (accessed January 2017).

32 ATSDR, Toxic Substances Portal: Substances A-Z, https://www.atsdr.cdc.gov/substances/indexAZ.asp, (accessed January 2017).

33 HSE, Chromium and You. Working with Chromium: Are you at Risk? HSE, 2013, http://www.hse.gov.uk/pubns/indg346.htm (accessed November 2016).

34 M. Valko, H. Morris and M. T. D. Cronin, Curr. Med. Chem., 2005, 12, 11611208.

35 I. Horvath and J. C. de Jongste, ed., Exhaled Biomarkers, European Respiratory Society, Sheffield, UK, 2010.

36 P. Kuban and F. Foret, Anal. Chim. Acta., 2013, 805, 1-18. 

W. C. Wilson, J. F. Swetland, J. L. Benumof, P. Laborde and R. Taylor, Anesthesiology., 1992, 76, 703-710.

S. Dodig and I. Cepelak, Biochem. Med., 2013, 23, 281-295. Pulm. Drug. Deliv., 2010, 23, 371-379.

S. Svensson, A. C. Olin, M. Larstad, G. Ljungkvist and K. Toren, J. Chromatogr. B: Anal. Technol. Biom. Life Sci., 2004, 809, 199-203.

T. Ueno, M. Kataoka, A. Hirano, K. lio, Y. Tanimoto, A. Kanehiro, C. Okada, R. Soda, K. Takahashi and M. Tanimoto, Respirol., 2008, 13, 654-663.

W. Formanek, D. Inci, R. P. Lauener, J. H. Wildhaber, U. Frey and G. L. Hall, Eur. Respir. J., 2002, 19, 487-491.

J. Liu and P. S. Thomas, Respiration., 2007, 74, 142-145.

K. Schwarz, H. Biller, H. Windt, W. Koch and J. M. Hohlfeld, J. Aerosol Med. Pulm. Drug Delivery., 2015, 28, 52-58.

A. A. Arif and C. Mitchell, J. Prim. Care Community Health., 2016, 7, 102-106.

K. Syslova, P. Kacer, M. Kuzma, V. Najmanova, Z. Fenclova, S. Vlckova, J. Lebedova and D. Pelclova, J. Chromatogr. B: Anal. Technol. Biom. Life Sci., 2009, 877, 2477-2486.

D. Pelclova, Z. Fenclova, P. Kacer, T. Navratil, M. Kuzma, J. Lebedova and P. Klusackova, Ind. Health., 2007, 45, 766-774.

49 K. Koskela, P. Oksa, R. Sauni, M. Linnainmaa, P. Toivio, L. Lehtimaeki, E. Moilanen, R. Nieminen, R. Luukkonen and J. Uitti, J. Occup. Environ. Med., 2015, 57, 124-128.

50 D. Pelclova, Z. Fenclova, S. VIckova, J. Lebedova, K. Syslova, O. Pecha, J. Belacek, T. Navratil, M. Kuzma and P. Kacer, Ind. Health., 2012, 50, 299-306.

51 D. Pelclova, Z. Fenclova, K. Syslova, S. Vlckova, J. Lebedova, O. Pecha, J. Belacek, T. Navratil, M. Kuzma and P. Kacer, Ind. Health., 2011, 49, 746-754.

52 G. E. Carpagnano, S. A. Kharitonov, O. Resta, M. P. Foschino-Barbaro, E. Gramiccioni and P. J. Barnes, Chest., 2003, 124, 1386-1392.

53 A. Zacharasiewicz, N. Wilson, C. Lex, A. Li, M. Kemp, J. Donovan, J. Hooper, S. A. Kharitonov and A. Bush, Pediatr. Pulmonol., 2004, 37, 273-275.

54 G. E. Carpagnano, P. J. Barnes, D. M. Geddes, M. E. Hodson and S. A. Kharitonov, Am. J. Respir. Crit. Care Med., 2003, 167, 1109-1112.

55 C. Gessner, H. Kuhn, H. J. Seyfarth, H. Pankau, J. Winkler, J. Schauer and H. Wirtz, Pneumologie. (Stuttgart, Germany)., 2001, 55, 414-419.

56 G. E. Carpagnano, O. Resta, M. P. Foschino-Barbaro, E. Gramiccioni and F. Carpagnano, Int. J. Biol. Markers, 2002, 17, 141-145.

57 C. Desvergne, M. Dubosson, M. Lacombe, V. Brun and V. Mossuz, J. Phys.: Conf. Ser., 2015, 617. 

Felten, T. Bruning, M. Raulf and J. Bunger, Adv. Exp. Med. Biol., 2015, 858, 5767.

M. Goldoni, M. Corradi, P. Mozzoni, G. Folesani, R. Alinovi, S. Pinelli, R. Andreoli, D. Pigini, R. Tillo, A. Filetti, C. Garavelli and A. Mutti, J. Breath Res., 2013, 7, 017101.

60 P. Montuschi, S. Martello, M. Felli, C. Mondino and M. Chiarotti, Rapid Commun. Mass Spectrom., 2004, 18, 2723-2729.

61 P. D. Boyce, J. Y. Kim, D. N. Weissman, J. Hunt and D. C. Christiani, J. Occup. Environ. Med., 2006, 48, 353-356.

62 H. Graczyk, N. Lewinski, J. Zhao, J.-J. Sauvain, G. Suarez, P. Wild, B. Danuser and M. Riediker, Part. Fibre Toxicol., 2016, 13, 31.

63 J. J. Sauvain, M. S. S. Hohl, P. Wild, J. A. Pralong and M. Riediker, J. Aerosol Med. Pulm. Drug Deliv., 2014, 27, 449-458.

64 P. Rosias, J. Breath Res., 2012, 6, 027102.

65 M. Goldoni, A. Caglieri, D. Poli, M. V. Vettori, M. Corradi, P. Apostoli and A. Mutti, Anal. Chim. Acta., 2006, 562, 229-235.

66 A. Caglieri, M. Goldoni, O. Acampa, R. Andreoli, M. V. Vettori, M. Corradi, P. Apostoli and A. Mutti, Environ. Health Perspect., 2006, 114, 542-546.

67 M. Goldoni, A. Caglieri, G. De Palma, O. Acampa, P. Gergelova, M. Corradi, P. Apostoli and A. Mutti, J. Environ. Monit., 2010, 12, 442-447.

68 F. Hoffmeyer, T. Weiss, M. Lehnert, B. Pesch, H. Berresheim, J. Henry, M. RaulfHeimsoth, H. C. Broding, J. Buenger, V. Harth and T. Bruening, J. Environ. Monit., 2011, 13, 212-218.

69 M. Gube, J. Ebel, P. Brand, T. Goeen, K. Holzinger, U. Reisgen and T. Kraus, Int. Arch. Occup. Environ. Health., 2010, 83, 803-811.

70 S. Hulo, A. Radauceanu, N. Cherot-Kornobis, M. Howsam, V. Vacchina, B. de, V, D. Rousset, M. Grzebyk, M. Dziurla, A. Sobaszek and J. L. Edme, Int. J. Hyg. Environ. Health., 2016, 219, 40-47.

71 P. M. Felix, S. M. Almeida, C. Franco, A. B. Almeida, C. Lopes, M. I. Claro, E. Fragoso, C. Teles, H. T. Wolterbeek and T. Pinheiro, Int. J. Environ. Health Res., 2015, 25, 67-80.

72 M. Goldoni, s. catalani, G. De Palma, P. manini, O. Acampa, M. Corradi, R. Bergonzi, P. Apostoli and A. Mutti, Environ. Health Perspect., 2004, 112, 12931298.

73 H. C. Broding, B. Michalke, T. Goen and H. Drexler, Int. Arch. Occup. Environ. Health., 2009, 82, 565-573.

74 S. Hulo, N. Cherot-Kornobis, M. Howsam, S. Crucq, B. de, V, A. Sobaszek and J. L. Edme, Toxicol. Lett., 2014, 226, 63-69. 

Freitas and S. M. Almeida, J. Radioanal. Nucl. Chem., 2013, 297, 377-382. P. M. Felix, C. Franco, M. A. Barreiros, B. Batista, S. Bernardes, S. M. Garcia, A. B. Almeida, S. M. Almeida, H. T. Wolterbeek and T. Pinheiro, Arch. Environ. Occup. Health., 2013, 68, 72-79.

77 J. Hunt, Immunol. Allergy Clin. North Am., 2007, 27, 587-596.

78 J. Hunt, J. Allergy Clin. Immunol., 2002, 110, 28-34.

79 M. Goldoni, A. Caglieri, R. Andreoli, D. Poli, P. Manini, M. V. Vettori, M. Corradi and A. Mutti, BMC Pulm. Med., 2005, 5, 10.

80 E. Dompeling, P. P. R. Rosias and Q. Jobsis, in Exhaled Biomarkers, ed. I. Horvath and J. C. De Jongste, European Respiratory Society, Sheffield, 2010, vol. 49, ch. 11, pp. 152-161.

81 I. Horvath, J. Hunt, P. J. Barnes, K. Alving, A. Antczak, E. Baraldi, G. Becher, W. J. van Beurden, M. Corradi, R. Dekhuijzen, R. A. Dweik, T. Dwyer, R. Effros, S. Erzurum, B. Gaston, C. Gessner, A. Greening, L. P. Ho, J. Hohlfeld, Q. Jobsis, D. Laskowski, S. Loukides, D. Marlin, P. Montuschi, A. C. Olin, A. E. Redington, P. Reinhold, E. L. van Rensen, I. Rubinstein, P. Silkoff, K. Toren, G. Vass, C. Vogelberg and H. Wirtz, Eur. Respir. J., 2005, 26, 523-548.

82 G. R. Johnson and L. Morawska, J. Aerosol Med. Pulm. Drug Deliv., 2009, 22, 229-237.

83 P. P. R. Rosias, E. Dompeling, H. J. E. Hendriks, J. Heijnens, R. Donckerwolcke and Q. Jobsis, Pediatr. Allergy Immunol., 2004, 15, 4-19.

84 P. Kuban, E.-G. Kobrin and M. Kaljurand, J. Chromatogr. A., 2012, 1267, 239245.

85 K. Moon, E. Guallar and A. Navas-Acien, Curr. Atheroscler. Rep., 2012, 14, $542-$ 555.

86 C. G. Sartal, M. D. Barciela-Alonso and P. Bermejo-Barrera, Microchem. J., 2012, 105, 65-71.

87 W. J. Seow, W. C. Pan, M. L. Kile, A. A. Baccarelli, Q. Quamruzzaman, M. Rahman, G. Mahiuddin, G. Mostofa, X. H. Lin and D. C. Christiani, Environ. Health Perspect., 2012, 120, 1733-1738.

88 F. Hoffmeyer, V. Harth, R. Merget, N. Goldscheid, E. Heinze, P. Degens, B. Pesch, J. Buenger, T. Bruening and M. Raulf-Heimsoth, J. Physiol. Pharmacol., 2007, 58, 289-298.

89 J. B. McCafferty, T. A. Bradshaw, S. Tate, A. P. Greening and J. A. Innes, Thorax., 2004, 59, 694-698.

90 T. M. Dwyer, Lung, 2004, 182, 241-250.

91 R. M. Effros, K. W. Hoagland, M. Bosbous, D. Castillo, B. Foss, M. Dunning, M. Gare, W. Lin and F. Sun, Am. J. Respir. Crit. Care Med., 2002, 165, 663-669. 

1505.

WHO, Environmental Health Criteria 224: Arsenic and Arsenic Compounds, World Health Organisation, Geneva, $2^{\text {nd }}$ edn, 2001. http://www.who.int/ipcs/publications/ehc/ehc_224/en/ (accessed May 2017). B. K. Mandal and K. T. Suzuki, Talanta, 2002, 58, 201-235.

95 R. Raml, W. Goessler and K. A. Francesconi, J. Chromatogr. A., 2006, 1128, 164170.

96 H. R. Hansen, A. Raab, K. A. Francesconi and J. Feldmann, Environ. Sci. Technol., 2003, 37, 845-851.

97 NTP, $14^{\text {th }}$ Report on Carcinogens, U.S. Department of Health and Human Services, U.S., 2016. http://ntp.niehs.nih.gov/go/roc14/ (accessed May 2017). B. A. Fowler, C. H. S. J. Chou, R. L. Jones and C. J. Chen, in Handbook on the Toxicology of Metals, ed. G. F. Nordberg, B. A. Fowler, M. Nordberg and L. T. Friberg, Elsevier, London, 3rd Edn, 2007, ch. 19, pp. 367-406.

99 J. Emsley, ed., Nature's Building Blocks: An A-Z Guide to the Elements, Oxford University Press, Oxford, 2003.

100 E. Dopp, A. D. Klingerman and R. A. Diaz-Bone, in Organometallics in Environment and Toxicology: Metal lons in Life Sciences, ed. A. Sigel, H. Sigel and R. K. O. Sigel, RSC Publishing, 2010, ch. 7, pp. 231 - 265.

101 ATSDR, Toxicological Profile for Arsenic, Department of Health and Human Services, Atlanta, 2007.

https://www.atsdr.cdc.gov/ToxProfiles/tp.asp?id=22\&tid=3\#bookmark04 (accessed February 2017).

102 J. C. Saha, A. K. Dikshit, M. Bandyopadhyay and K. C. Saha, Crit. Rev. Environ. Sci. Technol., 1999, 29, 281-313.

103 G. A. Levvy, Brit. J. Pharmacol., 1946, 1, 287-290.

104 J. W. Harrisson, E. W. Packman and D. D. Abbott, AMA Arch. Ind. Health, 1958, 17, 118-123.

105 T. Kaise, S. Watanabe and K. Itoh, Chemosphere, 1985, 14, 1327-1332.

106 X.C. Le and M. Ma, J. chromatogr. A., 1997, 764, 55-64.

107 T. B. Gaines, Toxicol. Appl. Pharmacol., 1960, 2, 88-99.

108 T. Kaise, H. Yamauchi, Y. Horiguchi, T. Tani, S. Watanabe, T. Hirayama and S. Fukui, Appl. Organomet. Chem., 1989, 3, 273-277.

109 IARC, IARC Monographs on the Evaluation of Carcinogenic Risks to Humans, Volume 100C: A Review of Human Carcinogens: Arsenic, Metals, Fibres and Dusts, 2012, International Agency for Research on Cancer, Lyon, France. http://monographs.iarc.fr/ENG/Monographs/vol100C/index.php (accessed May 2017). 
128 K. A. Francesconi, Pure Appl. Chem., 2010, 82, 373-381.

129 C. Niegel and F. M. Matysik, Anal. Chim. Acta, 2010, 657, 83-99.

130 J. Feldmann and E. M. Krupp, Anal. Bioanal. Chem, 2011, 399, 1735-1741.

131 K. A. Francesconi, R. Tanggaar, C. J. McKenzie and W. Goessler, Clin. Chem, 2002, 48, 92-101.

132 R. Raml, G. Raber, A. Rumpler, T. Bauernhofer, W. Goessler and K. A. Francesconi, Chem. Res. Toxicol., 2009, 22, 1534-1540.

133 R. Raml, W. Goessler, P. Traar, T. Ochi and K. A. Francesconi, Chem. Res. Toxicol., 2005, 18, 1444-1450. 
134 C. Newcombe, A. Raab, P. N. Williams, C. Deacon, P. I. Haris, A. A. Meharg and J. Feldmann, J. Environ. Monit., 2010, 12, 832-837.

135 V. Taylor, B. Goodale, A. Raab, T. Schwerdtle, K. Reimer, S. Conklin, M. R. Karagas and K. A. Francesconi, Sci. Total Environ., 2017, 580, 266-282.

136 H. R. Hansen, R. Pickford, J. Thomas-Oates, M. Jaspars and J. Feldmann, Angew. Chem. Int. Ed., 2004, 43, 337-340.

137 M. Molin, S. M. Ulven, H. M. Meltzer and J. Alexander, J. Trace Elem. Med. Biol., 2015, 31, 249-259.

138 H. R. Hansen, A. Raab and J. Feldmann, J. Anal. At. Spectrom., 2003, 18, 474479.

139 E. Schmeisser, W. Goessler and K. A. Francesconi, Anal. Bioanal. Chem., 2006, 385, 367-376.

140 B. K. Mandal, K. T. Suzuki, K. Anzai, K. Yamaguchi and Y. Sei, J. Chromatogr. B: Anal. Technol. Biomed. Life Sci., 2008, 874, 64-76.

141 R. Raml, A. Rumpler, W. Goessler, M. Vahter, L. Li, T. Ochi and K. A. Francesconi, Toxicol. Appl. Pharmacol., 2007, 222, 374-380.

142 L. Leffers, F. Ebert, M. S. Taleshi, K. A. Francesconi and T. Schwerdtle, Mol. Nutr. Food Res., 2013, 57, 1270-1282.

143 P. Andrewes, D. M. Demarini, K. Funasaka, K. Wallace, V. W. M. Lai, H. S. Sun, W. R. Cullen and K. T. Kitchin, Environ. Sci. Technol., 2004, 38, 4140-4148.

144 F. Ebert, S. Meyer, L. Leffers, G. Raber, K. A. Francesconi and T. Schwerdtle, J. Trace Elem. Med. Biol., 2016, 38, 150-156.

145 S. Meyer, M. Matissek, S. M. Mueller, M. S. Taleshi, F. Ebert, K. A. Francesconi and T. Schwerdtle, Metallomics, 2014, 6, 1023-1033.

146 S. Meyer, G. Raber, F. Ebert, L. Leffers, S. M. Mueller, M. S. Taleshi, K. A. Francesconi and T. Schwerdtle, Toxicol. Res., 2015, 4, 1289-1296.

147 F. Ebert, L. Leffers, T. Weber, S. Berndt, A. Mangerich, S. Beneke, A. Burkle and T. Schwerdtle, J. Trace Elem. Med. Biol, 2014, 28, 138-146.

148 H. Naranmandura, Y. Ogra, K. Iwata, J. Lee, K. T. Suzuki, M. Weinfeld and X. C. Le, Toxicol. Appl. Pharmacol., 2009, 238, 133-140.

149 H. Naranmandura, M. W. Carew, S. Xu, J. Lee, E. M. Leslie, M. Weinfeld and X. C. Le, Chem. Res. Toxicol., 2011, 24, 1586-1596.

150 M. Bartel, F. Ebert, L. Leffers, U. Karst and T. Schwerdtle, J. Toxicol., 2011, 2011, 373141.

151 H. Naranmandura, K. Ibata and K. T. Suzuki, Chem. Res. Toxicol, 2007, 20, 11201125.

152 M. Unterberg, L. Leffers, F. Hubner, H. U. Humpf, K. Lepikhov, J. Walter, F. Ebert and T. Schwerdtle, Toxicol. Res., 2014, 3, 456-464.

153 T. Ochi, K. Kita, T. Suzuki, A. Rumpler, W. Goessler and K. A. Francesconi, Toxicol. Appl. Pharmacol., 2008, 228, 59-67. 
154 H. Naranmandura, K. Iwata, K. T. Suzuki and Y. Ogra, Toxicol. Appl. Pharmacol., 2010, 245, 67-75.

155 H. Naranmandura, N. Suzuki, K. Iwata, S. Hirano and K. T. Suzuki, Chem. Res. Toxicol., 2007, 20, 616-624.

156 H. Naranmandura and K. T. Suzuki, Toxicol. Appl. Pharmacol., 2008, 227, 390399.

157 J. Morton and H. Mason, J. Anal. Toxicol., 2006, 30, 293-301.

158 B. Cleland, A. Tsuchiya, D. A. Kalman, R. Dills, T. M. Burbacher, J. W. White, E. M. Faustman and K. Marien, Environ. Health Perspect., 2009, 117, 632-638.

159 P. Alava, G. Du Laing, M. Odhiambo, A. Verliefde, F. Tack and T. R. Van de Wiele, J. Environ. Sci. Health, Part A: Toxic/Hazard. Subst. Environ. Eng., 2013, 48, 656-665.

160 D. Halder, A. Biswas, Z. Slejkovec, D. Chatterjee, J. Nriagu, G. Jacks and P. Bhattacharya, Sci. Total Environ., 2014, 497, 200-208.

161 A. H. Ackerman, P. A. Creed, A. N. Parks, M. W. Fricke, C. A. Schwegel, J. T. Creed, D. T. Heitkemper and N. P. Vela, Environ. Sci. Technol., 2005, 39, 52415246.

162 F. Cubadda, S. Ciardullo, M. D'Amato, A. Raggi, F. Aureli and M. Carcea, J. Agric. Food Chem., 2010, 58, 10176-10183.

163 A. Gonzalvez, A. Llorens, M. L. Cervera, S. Armenta and M. de la Guardia, Food Chem., 2009, 115, 360-364.

164 M. M. Nearing, I. Koch and K. J. Reimer, Environ. Sci. Technol., 2014, 48, 1420314210.

165 D. Wilson, J. Environ. Health., 2015, 78, 16-22.

166 J. H. Huang, K. N. Hu, J. Ilgen and G. Ilgen, Food Addit. Contam., Part A., 2012, 29, 85-93.

167 S. D. Conklin and P. E. Chen, Food Addit. Contam., Part A., 2012, 29, 1272-1279.

168 B. Sadee, M. E. Foulkes and S. J. Hill, J. Anal. At. Spectrom., 2015, 30, 102-118.

169 WHO, Guidelines for Drinking Water Quality: Fourth Edition Incorporating the First Addendum, World Health Organisation, Geneva, 2017.

http://www.who.int/water_sanitation_health/publications/gdwq4-with-add1chapters/en/ (accessed May 2017).

170 P. L. Smedley and D. G. Kinniburgh, Appl. Geochem., 2002, 17, 517-568.

171 WHO, Arsenic Fact Sheet, http://www.who.int/mediacentre/factsheets/fs372/en/ (accessed May 2017).

172 E. L. Ander, M. J. Watts, P. L. Smedley, E. M. Hamilton, R. Close, H. Crabbe, T. Fletcher, A. Rimell, M. Studden and G. Leonardi, Environ. Geochem. Health, 2016, 38, 1313-1332. 
173 D. R. S. Middleton, M. J. Watts, E. M. Hamilton, E. L. Ander, R. M. Close, K. S. Exley, H. Crabbe, G. S. Leonardi, T. Fletcher and D. A. Polya, Sci. Rep., 2016, 6, 25656.

174 BBC News, Iceman's Final Meal, 2002, http://news.bbc.co.uk/1/hi/sci/tech/2262615.stm, (accessed November 2016).

175 J. W. Yager, J. B. Hicks and N. Fabianova, Environ. Health Perspect., 1997, 105, 836-842.

176 M. Vahter, L. Friberg, B. Rahnster, A. Nygren and P. Nolinder, Int. Arch. Occup. Environ. Health, 1986, 57, 79-91.

177 S. S. Pinto, M. O. Varner, K. W. Nelson, A. L. Labbe and L. D. White, J. Occup. Environ. Med., 1976, 18, 677-680.

178 J. Scancar and R. Milacic, J. Anal. At. Spectrom., 2014, 29, 427-443.

179 R. Rakhunde, L. Deshpande and H. D. Juneja, Crit. Rev. Environ. Sci. Technol., 2012, 42, 776-810.

180 P. C. Grevatt, EPA Toxicological Review of Trivalent Chromium, US Environmental Protection Agency, Washington DC, 1998.

https://cfpub.epa.gov/ncea/iris/iris documents/documents/toxreviews/0028tr .pdf, (accessed February 2017).

181 D. G. Barceloux, J. Toxicol., Clin. Toxicol., 1999, 37, 173-194

182 K. V. Iserson, W. Banner, R. C. Froede and M. R. Derrick, J. Emerg. Med., 1983, 1, 143-149.

183 D. J. Paustenbach, B. L. Finley, F. S. Mowat and B. D. Kerger, J. Toxicol. Environ. Health A., 2003, 66, 1295-1339.

184 A. Zhitkovich, Chem. Res. Toxicol., 2011, 24, 1617-1629.

185 L. A. Saryan and M. Reedy, J. Anal. Toxicol., 1988, 12, 162-164.

186 P. P. Varma, V. Jha, A. K. Ghosh, K. Joshi and V. Sakhuja, Renal Failure, 1994, 16, 653-657.

187 J. P. Goulle, E. Saussereau, J. Grosjean, C. Doche, L. Mahieu, J. M. Thouret, M. Guerbet and C. Lacroix, Forensic Sci. Int, 2012, 217, e8-e12.

188 S. C. Gad, W. J. Powers, B. J. Dunn, G. M. Hoffman, K. M. Siino and R. D. Walsh in Chromium Symposium 1986: An Update, Industrial Health Foundation, Pittsburgh, 1986, pp.43-58.

189 E. H. Vernot, J. D. MacEwan, C. C. Haun and E. R. Kinkead, Toxicol. Appl. Pharmacol., 1977, 42, 417-423.

190 Y. Suzuki and K. Fukuda, Arch. Toxicol., 1990, 64, 169-176.

191 A. M. Standeven and K. E. Wetterhahn, Carcinog., 1992, 13, 1319-1324.

192 P. Hoet, in Handbook of Elemental Speciation II: Species in the Environment, Food, Medicine and Occupational Health, ed. R. Cornelis, J. A. Caruso, H. Crews and K. Heumann, Wiley - Blackwell, 2005, ch. 2.6.2, pp. 136-157. 
193 R. Sedman, J. Beaumont, J. Budroe and C. Vidair, Public Health Goals for Chemicals in Drinking Water: Hexavalent Chromium (CR VI), Office of Environmental Health Hazard Assessment, California, 2011. https://oehha.ca.gov/water/public-health-goals-phgs (accessed February 2017).

194 NIOSH, Criteria for a Recommended Standard: Occupational Exposure to Hexavalent Chromium, National Institute of Occupational Safety and Health, Atlanta, 2013. https://www.cdc.gov/niosh/docs/2013-128/ (accessed February 2017).

195 IARC, IARC Monographs on the Evaluation of Carcinogenic Risks to Humans, Volume 49: Chromium, Nickel and Welding, International Agency for Research on Cancer, Lyon, 1990.

http://monographs.iarc.fr/ENG/Monographs/vol49/index.php (accessed February 2017).

196 R. Saha, R. Nandi and B. Saha, J. Coord. Chem., 2011, 64, 1782-1806.

197 E. Lindberg and O. Vesterberg, Am. J. Ind. Med., 1989, 16, 485-492.

198 R. Petersen, J. F. Thomsen, N. K. Jorgensen and S. Mikkelsen, Occup. Environ. Med., 2000, 57, 140-142.

199 S. De Flora, Carcinog., 2000, 21, 533-541.

200 IARC, IARC Monographs on the Evaluation of Carcinogenic Risks to Humans, Volume 68: Silica, Some Silicates, Coal Dust and para-Aramid Fibrils, International Agency for Research on Cancer, Lyon, 1997. http://monographs.iarc.fr/ENG/Monographs/vol68/index.php (accessed May 2017).

201 C. C. Leung, I. T. S. Yu and W. Chen, Lancet, 2012, 379, 2008-2018.

202 M. A. Farooq and K.-J. Dietz, Front. Plant Sci., 2015, 6, 994.

203 R. Jugdaohsingh, S. H. C. Anderson, K. L. Tucker, H. Elliott, D. P. Kiel, R. P. H. Thompson and J. J. Powell, Am. J. Clin. Nutr., 2002, 75, 887-893.

204 Institute of Medicine Panel on Micronutrients, Dietary References Intakes for Vitamin A, Vitamin K, Arsenic, Boron, Chromium, Copper, Iodine, Iron, Manganese, Molybdenum, Nickel, Silicon, Vanadium, and Zinc, National Academies Press, Washington, 2001. https://www.ncbi.nlm.nih.gov/books/NBK222310/ (accessed January 2017).

205 R. Jugdaohsingh, J. Nutr., Health \& Aging., 2007, 11, 99-110.

206 S. Sripanyakorn, R. Jugdaohsingh, H. Elliott, C. Walker, P. Mehta, S. Shoukru, R. P. H. Thompson and J. J. Powell, Br. J. Nutr., 2004, 91, 403-409.

207 OSHA, Crystalline Silica Exposure Health Hazard Information Fact Sheet, Occupational Safety and Health Administration, Washington, 2002, https://www.osha.gov/pls/publications/publication.athruz?pType=Industry\&pl $\underline{D=192}$ (accessed May 2017). 
$208 \mathrm{NIOSH}$, Health Effects of Occupational Exposure to Respirable Crystalline Silica, National Institute for Occupational Safety and Health, Cincinnati, 2002, https://www.cdc.gov/niosh/docs/2002-129/ (accessed May 2017).

209 T. Norboo, P. T. Angchuk, M. Yahya, S. R. Kamat, F. D. Pooley, B. Corrin, I. H. Kerr, N. Bruce and K. P. Ball, Thorax, 1991, 46, 341-343.

210 NHS, Silicosis, http://www.nhs.uk/Conditions/Silicosis/Pages/Introduction.aspx, (accessed May 2017).

211 CDC, Fourth National Report on Human Exposure to Environmental Chemicals: Updated Tables January 2017, Volume 1, Centers for Disease Control and Prevention, Atlanta, 2017. https://www.cdc.gov/exposurereport/ (accessed May 2017).

212 M. I. Greenberg, J. Waksman and J. Curtis, DM, Dis. -Mon., 2007, 53, 394-416.

213 M. R. Kramer, P. D. Blanc, E. Fireman, A. Amital, A. Guber, N. A. Rhahman and D. Shitrit, Chest, 2012, 142, 419-424.

214 A. Perez-Alonso, J. Antonio Cordoba-Dona, J. Luis Millares-Lorenzo, E. FigueroaMurillo, C. Garcia-Vadillo and J. Romero-Morillo, Int. J. Occup. Environ. Health, 2014, 20, 26-32.

215 S. Pascual, I. Urrutia, A. Ballaz, L. Arrizubieta, L. Altube and C. Salinas, Arch. Bronconeumol., 2011, 47, 50-51.

216 G. K. Friedman, R. Harrison, H. Bojes, K. Worthington and M. Filios, MmwrMorbidity Mort. Wkly Rep., 2015, 64, 129-130.

217 V. Paolucci, R. Romeo, A. G. Sisinni, D. Bartoli, M. A. Mazzei and P. Sartorelli, Arch. Bronconeumol., 2015, 51, e57-e60.

218 B. Widner, A. Mirlach, A. Hausen, I. Jenewein, D. Jenewein, M. P. Dierich, H. Wachter and D. Fuchs, Clin. Chim. Acta, 1998, 277, 51-63.

219 R. Kobayashi, S. Okamura, K. Yamada and M. Kudo, Anal. Sci., 1997, 13, 17-20.

220 J. Takala, presented in part at Epidemiology in Occupational Health Conference, Barcelona, September, 2016.

221 L. Rushton, S. Bagga, R. Bevan, T. Brown, J. Cherrie, P. Holmes, L. Fortunato, S. Hutchings, R. Slack, M. V. Tongeren, C. Young and G. S. Evans, The burden of occupational cancer in Great Britain: Overview Report, HSE Books, 2012. http://www.hse.gov.uk/research/rrhtm/rr931.htm (accessed May 2017).

222 D. M. Templeton, F. Ariese, R. Cornelis, L. G. Danielsson, H. Muntau, H. P. Van Leeuwen and R. Lobinski, Pure Appl. Chem., 2000, 72, 1453-1470.

223 E. Krupp, F. Seby, R. Rodriguez Martin-Doimeadios, A. Holliday, M. Moldovan, G. Kollensperger, S. Hann and O. F. X. Donard, in ICP Mass Spectrometry Handbook, ed. S. M. Nelms, Blackwell Publishing, Oxford, 2005, ch. 7, pp. 259335.

224 J. J. Thompson and R. S. Houk, Anal. Chem., 1986, 58, 2541-2548. 
225 M. Montes-Bayon, K. DeNicola and J. A. Caruso, J. Chromatogr. A, 2003, 1000, 457-476.

226 C. A. Ponce de Leon, M. Montes-Bayon and J. A. Caruso, J. Chromatogr. A, 2002, 974, 1-21.

227 P. R. Haddad and P. E. Jackson, Ion Chromatography: Principles and Applications, Elsevier Science Publishers, Amsterdam, 1990.

228 R. S. Houk, V. A. Fassel, G. D. Flesch, H. J. Svec, A. L. Gray and C. E. Taylor, Anal. Chem., 1980, 52, 2283-2289.

229 A. Montaser, J. A. McLean, H. Liu and J.-M. Mermet, in Inductively Couple Plasma Mass Spectrometry, ed. A. Montaser, Wiley-VCH, New York, 1998, pp. 1-31.

230 S. J. Hill, Fisher A., Liezers, M., in ICP Mass Spectrometry Handbook, ed. S. M. Nelms, Blackwell Publishing, Oxford, 2005, ch. 1, pp. 1-25.

231 J. J. L. Todoli and J.-M. Mermet, in Elemental and Isotope Ratio Mass Spectrometry, ed. D. Beauchemin and D. E. Matthews, Elsevier, Amsterdam, 2010, vol. 5, ch. 1, pp. 11-22.

232 M. Liezers, in Elemental and Isotope Ratio Mass Spectrometry, ed. D. Beauchemin and D. E. Matthews, Elsevier Amsterdam, 2010, vol. 5, ch. 1, pp. 102-110.

233 C. Degueldre and P. Y. Favarger, Colloids Surf., A, 2003, 217, 137-142.

234 A. Ulrich, S. Losert, N. Bendixen, A. Al-Kattan, H. Hagendorfer, B. Nowack, C. Adlhart, J. Ebert, M. Lattuada and K. Hungerbuehler, J. Anal. At. Spectrom., 2012, 27, 1120-1130.

235 R. Peters, Z. Herrera-Rivera, A. Undas, M. van der Lee, H. Marvin, H. Bouwmeester and S. Weigel, J. Anal. At. Spectrom., 2015, 30, 1274-1285.

236 F. Laborda, J. Jimenez-Lamana, E. Bolea and J. R. Castillo, J. Anal. At. Spectrom., 2011, 26, 1362-1371.

237 D. M. Mitrano, E. K. Lesher, A. Bednar, J. Monserud, C. P. Higgins and J. F. Ranville, Environ. Toxicol. Chem., 2012, 31, 115-121.

238 H. E. Pace, N. J. Rogers, C. Jarolimek, V. A. Coleman, C. P. Higgins and J. F. Ranville, Anal. Chem., 2011, 83, 9361-9369.

239 J. Tuoriniemi, G. Cornelis and M. Hassellov, Anal. Chem., 2012, 84, 3965-3972.

240 J. H. Batey, T. Prohaska, M. S. A. Horstwood, H. Goenaga-Infante and G. C. Eiden, in ICP Mass Spectrometry Handbook, ed. S. M. Nelms, Blackwell Publishing, Oxford, 2005, ch. 2, pp. 26-116.

241 S. J. Ray, in Elemental and Isotope Ratio Mass Spectrometry, ed. D. Beauchemin and D. E. Matthews, Elsevier, Amsterdam, 2010, vol. 5, ch. 1, p. 111-121.

242 J. C. Traeger, in Elemental and Isotope Ratio Mass Spectrometry, ed. D. Beauchemin and D. E. Matthews, Elsevier, Amsterdam, 2010, vol. 5, ch. 4, pp. 369-380. 
243 E. Leese, J. Morton, E. Tan, P. H. Gardiner and V. A. Carolan, J. Anal. Toxicol, 2014, 38, 24-30.

244 P. Heitland and H. D. Koester, J. Anal. Toxicol., 2008, 32, 308-314.

245 I. Navarro Serrano, M. T. Llorente Ballesteros, S. S. Fernandez Pacheco, S. Izquierdo Alvarez and J. L. Lopez Colon, Sci. Total Environ., 2016, 571, 164-171.

246 A. Hata, Y. Endo, Y. Nakajima, M. Ikebe, M. Ogawa, N. Fujitani and G. Endo, J. Occup. Health, 2007, 49, 217-223.

247 Health Canada, Third Report on Human Biomonitoring of Environmental Chemicals in Canada: Survey Cycle 3 (2012-2103), Health Canada, Ottawa, 2015. http://www.hc-sc.gc.ca/ewh-semt/pubs/contaminants/chms-ecmscycle3/index-eng.php (accessed May 2017).

248 C. Cascio, A. Raab, R. O. Jenkins, J. Feldmann, A. A. Meharg and P. I. Haris, J. Environ. Monit., 2011, 13, 257-265.

249 V. M. O. Carioni, J. A. McElroy, J. M. Guthrie, R. A. Ngwenyama and J. D. Brockman, Talanta, 2017, 165, 76-83.

250 M. Molin, T. A. Ydersbond, S. M. Ulven, M. Holck, L. Dahl, J. J. Sloth, D. Fliegel, W. Goessler, J. Alexander and H. M. Meltzer, Food Chem. Toxicol., 2012, 50, 2462-2472.

251 K. Yoshida, K. Kuroda, X. Zhou, Y. Inoue, Y. Date, H. Wanibuchi, S. Fukushima and G. Endo, Chem. Res. Toxicol, 2003, 16, 1124-1129.

252 K. M. Kubachka, M. C. Kohan, K. Herbin-Davis, J. T. Creed and D. J. Thomas, Toxicol. Appl. Pharmacol., 2009, 239, 137-143.

253 T. S. Pinyayev, M. J. Kohan, K. Herbin-Davis, J. T. Creed and D. J. Thomas, Chem. Res. Toxicol., 2011, 24, 475-477.

254 H. Naranmandura, N. Suzuki and K. T. Suzuki, Toxicol. Appl. Pharmacol., 2008, 231, 328-335.

255 D. J. Carlin, M. F. Naujokas, K. D. Bradham, J. Cowden, M. Heacock, H. F. Henry, J. S. Lee, D. J. Thomas, C. Thompson, E. J. Tokar, M. P. Waalkes, L. S. Birnbaum and W. A. Suk, Environ. Health Perspect., 2016, 124, 890-899.

256 C. Keen, E. Tan, J. McAlinden, P. Woolger and P. Smith, Exposure to Hexavalent Chromium, Nickel and Cadmium Compounds in the Electroplating Industry, HSE Books, 2013. http://www.sea.org.uk/health--safety/biological-monitoring/ (accessed May 2017).

257 Surface Engineering Association \& HSE, Hexavalent Chromium in Electroplating: Monitoring for Exposure, http://www.sea.org.uk/health--safety/hexavalentchrome/ (accessed March 2017).

258 Surface Engineering Association \& HSE, Hexavalent Chromium in Electroplating: Prevention and Control of Skin Exposure Risks, http://www.sea.org.uk/health-safety/hexavalent-chrome/ (accessed March 2017). 
259 Surface Engineering Association \& HSE, Hexavalent Chromium in Electroplating: Prevention and Control of Chromic Acid Mist, http://www.sea.org.uk/health-safety/hexavalent-chrome/ (accessed March 2017).

260 Surface Engineering Association \& HSE, Nickel and Nickel Alloy Plating Operations: Controlling the Risk of Skin Exposure, http://www.sea.org.uk/health--safety/nickel/ (accessed March 2017).

261 Surface Engineering Association \& HSE, Nickel and Nickel Alloy Plating Operations: Controlling the Inhalation Risk, http://www.sea.org.uk/health-safety/nickel// (accessed March 2017).

262 D. Bartczak and H. Goenaga-Infante, in Nanomaterial Characterization: An Introduction, ed. R. Tantra, Wiley \& Sons, 2016, ch. 4, pp. 63-80.

263 R. J. B. Peters, Z. H. Rivera, H. Bouwmeester, S. Weigel and H. J. P. Marvin, Qual. Assur. Saf. Crops Foods, 2014, 6, 281-290.

264 Malvern, Dynamic Light Scattering, http://www.malvern.com/en/products/technology/dynamic-lightscattering/default.aspx, (accessed May 2017).

265 Malvern, Nanoparticle Tracking Analysis, http://www.malvern.com/en/products/technology/nanoparticle-trackinganalysis/, (accessed May 2017).

266 IZON Science, Applications of Tunable Resistive Pulse Sensing, http://www.izon.com/applications/, (accessed May 2017).

267 CPS Instruments Europe, Disc Centrifuge, http://www.cpsinstruments.eu/centrifuge.html, (accessed May 2017).

268 O. Lozano, O. Toussaint, J. M. Dogne and S. Lucas, J. Phys.: Conf. Ser,. 2013, 429, 012010.

269 Australian Microscopy and Microanalysis Research Facility, MyScope: Applications and Practical Uses - What the TEM can do., http://www.ammrf.org.au/myscope/tem/background/practical/ (accessed May 2017).

270 M. Master, Transmission Electron Microscope: Uses in Microscopy Advantages and Disadvantages, http://www.microscopemaster.com/transmission-electronmicroscope.html, (accessed May 2017).

271 C. Degueldre and P. Y. Favarger, Talanta, 2004, 62, 1051-1054.

272 C. Degueldre, P. Y. Favarger and C. Bitea, Anal. Chim. Acta, 2004, 518, 137-142.

273 C. Degueldre, P. Y. Favarger, R. Rosse and S. Wold, Talanta, 2006, 68, 623-628.

274 C. Degueldre, P. Y. Favarger and S. Wold, Anal. Chim. Acta, 2006, 555, 263-268.

275 RIKILT - Institute of Food Safety, Nanoparticle Suspensions - Counting and Sizing Nanoparticles Via Single Particle Inductively Coupled Plasma Mass Spectrometry, 2013. https://www.wur.nl/en/show/Single-Particle-Calculationtool.htm (accessed February 2017). 
Version 2. https://www.wur.nl/en/show/Single-Particle-Calculation-tool.htm (accessed February 2017).

277 M. D. Montano, B. J. Majestic, A. K. Jamting, P. Westerhoff and J. F. Ranville, Anal. Chem., 2016, 88, 4733-4741.

278 J. W. Olesik and P. J. Gray, J. Anal. At. Spectrom., 2012, 27, 1143-1155.

279 S. Lee, X. Bi, R. B. Reed, J. F. Ranville, P. Herckes and P. Westerhoff, Environ. Sci. Technol., 2014, 48, 10291-10300.

280 F. Aureli, M. D'Amato, A. Raggi, F. Cubadda, P. Scardina and P. Planitz, Quantitative Characterisation of Silica Nanoparticles by Asymmetric Flow FFFMALS-ICP-QQQ Using the Agilent 8800 ICP-QQQ, 2016.

281 M. Yamanaka, T. Itagaki and S. Wilbur, Agilent Technologies, 2016.

282 F. Aureli, M. D'Amato, A. Raggi and F. Cubadda, J. Anal. At. Spectrom., 2015, 30, 1266-1273.

283 M. D. Montano, J. W. Olesik, A. G. Barber, K. Challis and J. F. Ranville, Anal. Bioanal. Chem., 2016, 408, 5053-5074.

284 F. Aureli, M. D'Amato, B. De Berardis, A. Raggi, A. C. Turco and F. Cubadda, J. Anal. At. Spectrom., 2012, 27, 1540-1548.

285 D. Bartczak, P. Vincent and H. Goenaga-Infante, Anal. Chem., 2015, 87, 54825485.

286 S. E. Mischler, E. G. Cauda, M. Di Giuseppe, L. J. McWilliams, C. St. Croix, M. Sun, J. Franks and L. A. Ortiz, J. Occup. Med. Toxicol., 2016, 11, 57.

287 G. Sirianni, H. D. Hosgood, III, M. D. Slade and J. Borak, J. Occup. Environ. Hyg., 2008, 5, 279-285.

288 G. Oberdorster, E. Oberdorster and J. Oberdorster, Environ. Health Perspect., 2005, 113, 823-839.

289 L. Leclerc, W. Rima, D. Boudard, J. Pourchez, V. Forest, V. Bin, P. Mowat, P. Perriat, O. Tillement, P. Grosseau, D. Bernache-Assollant and M. Cottier, Inhalation Toxicol., 2012, 24, 580-588.

290 J. H. Wiessner, N. S. Mandel, P. G. Sohnle and G. S. Mandel, Exp. Lung Res., 1989, 15, 801-812.

291 T. Kajiwara, A. Ogami, H. Yamato, T. Oyabu, Y. Morimoto and I. Tanaka, J. Occup. Health, 2007, 49, 88-94.

292 J. J. Wang, B. J. S. Sanderson and H. Wang, Environ. Mol. Mutagen., 2007, 48, 151-157.

293 R. Sauni, P. Oksa, L. Lehtimaki, P. Toivio, P. Palmroos, R. Nieminen, E. Moilanen and J. Uitti, Occup. Environ. Med., 2012, 69, 256-260.

294 M. J. Z. Sakhvidi, J. B. Ardekani, A. Firoozichahak, J. Zavarreza, M. Hajaghazade, M. Mostaghaci, A. Mehrparvar and A. Barkhordari, Int. J. Occup. Med. Environ. Health, 2015, 28, 81-89. 
295 J. J. Sauvain, G. Suarez, J. L. Edme, O. M. P. A. Bezerra, K. G. Silveira, L. S. Amaral, A. P. S. Carneiro, N. Cherot-Kornobis, A. Sobaszek and S. Hulo, J. Breath Res., 2017, 11, 016010.

296 M. Roman, C. Rigo, H. Castillo-Michel, I. Munivrana, V. Vindigni, I. Micetic, F. Benetti, L. Manodori and W. R. L. Cairns, Anal. Bioanal. Chem., 2016, 408, 51095124.

297 M. A. Gomez-Gonzalez, E. Bolea, P. A. O'Day, J. Garcia-Guinea, F. Garrido and F. Laborda, Anal. Bioanal. Chem., 2016, 408, 5125-5135.

298 A. R. Donovan, C. D. Adams, Y. F. Ma, C. Stephan, T. Eichholz and H. L. Shi, Anal. Bioanal. Chem., 2016, 408, 5137-5145. 


\section{Word Count}

Chapter 1 (Introduction): 16648

Chapter 2: 6983

Chapter 3: 5128

Chapter 4: 4531

Chapter 5: $\quad 6415$

Chapter 6: 2066

Chapter 7 (Discussion \& conclusion): 7058

Chapter 8 (Presentation \& publication list): 753

Total word count: 49582 


\section{Appendix A}

Duncan, A. Taylor, A. Leese, E. Allen, S. Morton, J. McAdam, J. Homicidal arsenic poisoning. Ann. Clin. Biochem., (2014) 52(4): 510-515. 